\title{
Traveling Quasi-periodic Water Waves with Constant Vorticity
}

\author{
M. Berti, L. Franzoi \& A. Masperoø
}

Communicated by P. RABINOWITZ

\begin{abstract}
We prove the first bifurcation result of time quasi-periodic traveling wave solutions for space periodic water waves with vorticity. In particular, we prove the existence of small amplitude time quasi-periodic solutions of the gravity-capillary water waves equations with constant vorticity, for a bidimensional fluid over a flat bottom delimited by a space-periodic free interface. These quasi-periodic solutions exist for all the values of depth, gravity and vorticity, and restrict the surface tension to a Borel set of asymptotically full Lebesgue measure.
\end{abstract}

\section{Contents}

1. Introduction and Main Result _ . . . . . . . . . . . . . . . . . . . . 100

2. Hamiltonian Structure and Linearization at the Origin . . . . . . . . . . . . 108

2.1. Hamiltonian Structure . . . . . . . . . . . . . . . . . . . . . . . 108

2.2. Linearization at the Equilibrium . . . . . . . . . . . . . . . . . . 111

2.3. Tangential and Normal Subspaces of the Phase Space . . . . . . . . . . . 115

3. Functional Setting . . . . . . . . . . . . . . . . . . . . . . . . 119

3.1. Pseudodifferential Calculus . . . . . . . . . . . . . . . . . . . . 123

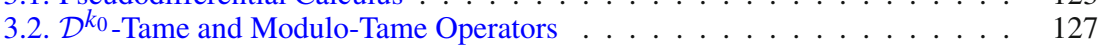

3.3. Hamiltonian and Reversible Operators . . . . . . . . . . . . . . . . . . . . 129

3.4. Momentum Preserving Operators . . . . . . . . . . . . . . . . . . . . . . 132

4. Transversality of Linear Frequencies . . . . . . . . . . . . . . . . . . . 135

5. Nash-Moser Theorem and Measure Estimates . . . . . . . . . . . . . . . . . . 142

5.1. Nash-Moser Theorem of Hypothetical Conjugation . . . . . . . . . . . . . 143

5.2. Measure Estimates . . . . . . . . . . . . . . . . . . . . . . . . . 145

6. Approximate Inverse . . . . . . . . . . . . . . . . . . . 150

7. The Linearized Operator in the Normal Subspace . . . . . . . . . . . . . . . . 159

7.1. Quasi-periodic Reparametrization of Time . . . . . . . . . . . . . . . . . 162

7.2. Linearized Good Unknown of Alinhac . . . . . . . . . . . . . . . . . . . . 164

7.3. Symmetrization and Reduction of the Highest Order . . . . . . . . . . . 165

7.4. Symmetrization up to Smoothing Remainders . . . . . . . . . . . . . . 173

7.5. Reduction of the Order $1 \ldots \ldots$. . . . . . . . . . . . . 176 
7.6. Reduction of the Order $1 / 2 \ldots \ldots \ldots$

7.7. Conclusion: Partial Reduction of $\mathcal{L}_{\omega} \ldots \ldots \ldots$. . . . . . . . . . . 184

8. Almost-Diagonalization and Invertibility of $\mathcal{L}_{\omega} \ldots \ldots \ldots \ldots \ldots$

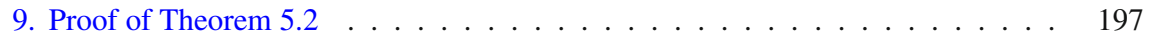

References . . . . . . . . . . . . . . . . . . . 200

\section{Introduction and Main Result}

The search for traveling surface waves in inviscid fluids is a very important problem in fluid mechanics, widely studied since the pioneering work of STOKES [38] in 1847. The existence of steady traveling waves, namely solutions which look stationary in a moving frame, either periodic or localized in space, is nowadays well understood in many different situations, mainly for bidimensional fluids.

On the other hand, the natural question regarding the existence of time quasiperiodic traveling waves - which cannot be reduced to steady solutions in a moving frame - has been not answered so far. This is the goal of the present paper. We consider space periodic waves. Major difficulties in this project concern the presence of "small divisors" and the quasi-linear nature of the equations. Related difficulties appear in the search of time periodic standing waves which have been constructed in the last few years in a series of papers by Iooss, Plotnikov, Toland [22,23, 25,34] for pure gravity waves, by ALAZARD-BALDI [1] in presence of surface tension and subsequently extended to time quasi-periodic standing waves solutions by BERTIMontalto [6] and Baldi-Berti-Haus-Montalto [2]. Standing waves are not traveling as they are even in the space variable. We also mention that all these recent results concern irrotational fluids.

In this paper we prove the first existence result of time quasi-periodic traveling wave solutions for the gravity-capillary water waves equations with constant vorticity for bidimensional fluids. The small amplitude solutions that we construct exist for any value of the vorticity (so also for irrotational fluids), any value of the gravity and depth of the fluid, and provided the surface tension is restricted to a Borel set of asymptotically full measure, see Theorem 1.5. For irrotational fluids the traveling wave solutions that we construct do not clearly reduce to the standing wave solutions in [6]. We remark that, in case of non zero vorticity, one cannot expect the bifurcation of standing waves since they are not allowed by the linear theory.

Before presenting in detail our main result, we introduce the water waves equations.

The water waves equations. We consider the Euler equations of hydrodynamics for a 2-dimensional perfect, incompressible, inviscid fluid with constant vorticity $\gamma$, under the action of gravity and capillary forces at the free surface. The fluid fills an ocean with depth $h>0$ (eventually infinite) and with space periodic boundary conditions, namely it occupies the region

$$
\mathcal{D}_{\eta, \mathrm{h}}:=\{(x, y) \in \mathbb{T} \times \mathbb{R}:-\mathrm{h} \leqq y<\eta(t, x)\}, \quad \mathbb{T}:=\mathbb{T}_{x}:=\mathbb{R} /(2 \pi \mathbb{Z})
$$


The unknowns of the problem are the divergence free velocity field $\left(\begin{array}{l}u(t, x, y) \\ v(t, x, y)\end{array}\right)$, which solves the Euler equation and the free surface $y=\eta(t, x)$ of the time dependent domain $\mathcal{D}_{\eta, \mathrm{h}}$. In case of a fluid with constant vorticity

$$
v_{x}-u_{y}=\gamma,
$$

the velocity field is the sum of the Couette flow $\left(\begin{array}{c}-\gamma y \\ 0\end{array}\right)$, which carries all the vorticity $\gamma$ of the fluid, and an irrotational field, expressed as the gradient of a harmonic function $\Phi$, called the generalized velocity potential.

Denoting by $\psi(t, x)$ the evaluation of the generalized velocity potential at the free interface $\psi(t, x):=\Phi(t, x, \eta(t, x))$, one recovers $\Phi$ by solving the elliptic problem

$$
\Delta \Phi=0 \text { in } \mathcal{D}_{\eta, \mathrm{h}}, \quad \Phi=\psi \text { at } y=\eta(t, x), \quad \Phi_{y} \rightarrow 0 \text { as } y \rightarrow-\mathrm{h} .
$$

The third condition in (1.2) means the impermeability property of the bottom

$$
\Phi_{y}(t, x,-\mathrm{h})=0, \text { if } \mathrm{h}<\infty, \quad \lim _{y \rightarrow-\infty} \Phi_{y}(t, x, y)=0, \text { if } \mathrm{h}=+\infty .
$$

Imposing that the fluid particles at the free surface remain on it along the evolution (kinematic boundary condition), and that the pressure of the fluid plus the capillary forces at the free surface is equal to the constant atmospheric pressure (dynamic boundary condition), the time evolution of the fluid is determined by the following system of equations (see $[8,42])$ :

$$
\left\{\begin{array}{l}
\eta_{t}=G(\eta) \psi+\gamma \eta \eta_{x} \\
\psi_{t}=-g \eta-\frac{\psi_{x}^{2}}{2}+\frac{\left(\eta_{x} \psi_{x}+G(\eta) \psi\right)^{2}}{2\left(1+\eta_{x}^{2}\right)}+\kappa\left(\frac{\eta_{x}}{\sqrt{1+\eta_{x}^{2}}}\right)_{x}+\gamma \eta \psi_{x}+\gamma \partial_{x}^{-1} G(\eta) \psi .
\end{array}\right.
$$

Here $g$ is the gravity, $\kappa$ is the surface tension coefficient, which we assume to belong to an interval $\left[\kappa_{1}, \kappa_{2}\right]$ with $\kappa_{1}>0$, and $G(\eta)$ is the Dirichlet-Neumann operator

$$
G(\eta) \psi:=G(\eta, \mathrm{h}) \psi:=\left.\sqrt{1+\eta_{x}^{2}}\left(\partial_{\vec{n}} \Phi\right)\right|_{y=\eta(x)}=\left.\left(-\Phi_{x} \eta_{x}+\Phi_{y}\right)\right|_{y=\eta(x)} .
$$

The water waves equations (1.3) are a Hamiltonian system that we describe in Section 2.1, and enjoy two important symmetries. First, they are time reversible: we say that a solution of (1.3) is reversible if

$$
\eta(-t,-x)=\eta(t, x), \quad \psi(-t,-x)=-\psi(t, x) .
$$

Second, since the bottom of the fluid domain is flat, the equations (1.3) are invariant by space translations. We refer to Section 2.1 for more details.

Let us comment shortly about the phase space of (1.3). As $G(\eta) \psi$ is a function with zero average, the quantity $\int_{\mathbb{T}} \eta(x) \mathrm{d} x$ is a prime integral of (1.3). Thus, with no loss of generality, we restrict to interfaces with zero spatial average $\int_{\mathbb{T}} \eta(x) \mathrm{d} x=0$. Moreover, since $G(\eta)[1]=0$, the vector field on the right hand side of (1.4) depends only on $\eta$ and $\psi-\frac{1}{2 \pi} \int_{\mathbb{T}} \psi \mathrm{d} x$. As a consequence, the variables $(\eta, \psi)$ of system 
(1.3) belong to some Sobolev space $H_{0}^{s}(\mathbb{T}) \times \dot{H}^{s}(\mathbb{T})$ for some $s$ large. Here $H_{0}^{s}(\mathbb{T})$, $s \in \mathbb{R}$, denotes the Sobolev space of functions with zero average

$$
H_{0}^{s}(\mathbb{T}):=\left\{u \in H^{s}(\mathbb{T}): \int_{\mathbb{T}} u(x) \mathrm{d} x=0\right\},
$$

and $\dot{H}^{s}(\mathbb{T}), s \in \mathbb{R}$, the corresponding homogeneous Sobolev space, namely the quotient space obtained by identifying all the $H^{s}(\mathbb{T})$ functions which differ only by a constant. For simplicity of notation we shall denote the equivalent class $[\psi]=$ $\{\psi+c, c \in \mathbb{R}\}$, just by $\psi$.

Linear water waves. When looking to small amplitude solutions of (1.3), a fundamental role is played by the system obtained linearizing (1.3) at the equilibrium $(\eta, \psi)=(0,0)$, namely

$$
\left\{\begin{array}{l}
\partial_{t} \eta=G(0) \psi \\
\partial_{t} \psi=-\left(g-\kappa \partial_{x}^{2}\right) \eta+\gamma \partial_{x}^{-1} G(0) \psi
\end{array}\right.
$$

The Dirichlet-Neumann operator at the flat surface $\eta=0$ is the Fourier multiplier

$$
G(0):=G(0, \mathrm{~h})=\left\{\begin{array}{ll}
D \tanh (\mathrm{h} D) & \text { if } \mathrm{h}<\infty \\
|D| & \text { if } \mathrm{h}=+\infty,
\end{array} \text { where } D:=\frac{1}{\mathrm{i}} \partial_{x},\right.
$$

with the symbol

$$
G_{j}(0):=G_{j}(0, \mathrm{~h})= \begin{cases}j \tanh (\mathrm{h} j) & \text { if } \mathrm{h}<\infty \\ |j| & \text { if } \mathrm{h}=+\infty .\end{cases}
$$

As we will show in Section 2.2, all reversible solutions (see (1.5)) of (1.6) are

$$
\begin{aligned}
\left(\begin{array}{l}
\eta(t, x) \\
\psi(t, x)
\end{array}\right) & =\sum_{n \in \mathbb{N}}\left(\begin{array}{c}
M_{n} \rho_{n} \cos \left(n x-\Omega_{n}(\kappa) t\right) \\
P_{n} \rho_{n} \sin \left(n x-\Omega_{n}(\kappa) t\right)
\end{array}\right) \\
& +\sum_{n \in \mathbb{N}}\left(\begin{array}{c}
M_{n} \rho_{-n} \cos \left(n x+\Omega_{-n}(\kappa) t\right) \\
P_{-n} \rho_{-n} \sin \left(n x+\Omega_{-n}(\kappa) t\right)
\end{array}\right),
\end{aligned}
$$

where $\rho_{n} \geqq 0$ are arbitrary amplitudes and $M_{n}$ and $P_{ \pm n}$ are the real coefficients

$$
M_{j}:=\left(\frac{G_{j}(0)}{\kappa j^{2}+g+\frac{\gamma^{2}}{4} \frac{G_{j}(0)}{j^{2}}}\right)^{\frac{1}{4}}, j \in \mathbb{Z} \backslash\{0\}, P_{ \pm n}:=\frac{\gamma}{2} \frac{M_{n}}{n} \pm M_{n}^{-1}, n \in \mathbb{N} .
$$

Note that the map $j \mapsto M_{j}$ is even. The frequencies $\Omega_{ \pm n}(\kappa)$ in (1.9) are

$$
\Omega_{j}(\kappa):=\sqrt{\left(\kappa j^{2}+g+\frac{\gamma^{2}}{4} \frac{G_{j}(0)}{j^{2}}\right) G_{j}(0)}+\frac{\gamma}{2} \frac{G_{j}(0)}{j}, \quad j \in \mathbb{Z} \backslash\{0\} .
$$

Note that the map $j \mapsto \Omega_{j}(\kappa)$ is not even due to the vorticity term $\gamma G_{j}(0) / j$, which is odd in $j$. Note that $\Omega_{j}(\kappa)$ actually depends also on the depth $h$, the gravity 
$g$ and the vorticity $\gamma$, but we highlight in (1.11) only its dependence with respect to the surface tension coefficient $\kappa$, since in this paper we shall move just $\kappa$ as a parameter to impose suitable non-resonance conditions; see Theorem 1.5. Other choices are possible.

All the linear solutions (1.9), depending on the irrationality properties of the frequencies $\Omega_{ \pm n}(\kappa)$ and the number of non zero amplitudes $\rho_{ \pm n}>0$, are either time periodic, quasi-periodic or almost-periodic. Note that the functions (1.9) are the linear superposition of plane waves traveling either to the right or to the left.

Remark 1.1. Actually, (1.9) contains also standing waves, for example when the vorticity $\gamma=0$ (which implies $\Omega_{-n}(\kappa)=\Omega_{n}(\kappa), P_{-n}=-P_{n}$ ) and $\rho_{-n}=\rho_{n}$, giving solutions even in $x$. This is the well known superposition effect of waves with the same amplitude, frequency and wavelength traveling in opposite directions.

Main result. We first provide the notion of quasi-periodic traveling wave.

Definition 1.2. (Quasi-periodic traveling wave) We say that $(\eta(t, x), \psi(t, x))$ is a time quasi-periodic traveling wave with irrational frequency vector $\omega=$ $\left(\omega_{1}, \ldots, \omega_{\nu}\right) \in \mathbb{R}^{v}, v \in \mathbb{N}$, that is $\omega \cdot \ell \neq 0, \forall \ell \in \mathbb{Z}^{v} \backslash\{0\}$, and "wave vectors" $\left(j_{1}, \ldots, j_{v}\right) \in \mathbb{Z}^{v}$, if there exist functions $(\breve{\eta}, \breve{\psi}): \mathbb{T}^{v} \rightarrow \mathbb{R}^{2}$ such that

$$
\left(\begin{array}{l}
\eta(t, x) \\
\psi(t, x)
\end{array}\right)=\left(\begin{array}{c}
\breve{\eta}\left(\omega_{1} t-j_{1} x, \ldots, \omega_{\nu} t-j_{v} x\right) \\
\breve{\psi}\left(\omega_{1} t-j_{1} x, \ldots, \omega_{\nu} t-j_{\nu} x\right)
\end{array}\right) .
$$

Remark 1.3. If $v=1$, such functions are time periodic and indeed stationary in a moving frame with speed $\omega_{1} / j_{1}$. On the other hand, if the number of frequencies $v$ is $\geqq 2$, the waves $(1.12)$ cannot be reduced to steady waves by any appropriate choice of the moving frame.

In this paper we shall construct traveling quasi-periodic solutions of (1.3) with a diophantine frequency vector $\omega$ belonging to an open bounded subset $\Omega$ in $\mathbb{R}^{v}$, namely, for some $v \in(0,1), \tau>v-1$, with $\Omega \subset \mathbb{R}^{v}$,

$$
\operatorname{DC}(v, \tau):=\left\{\omega \in \Omega:|\omega \cdot \ell| \geqq v\langle\ell\rangle^{-\tau}, \forall \ell \in \mathbb{Z}^{v} \backslash\{0\},\langle\ell\rangle:=\max \{1,|\ell|\}\right\} .
$$

Regarding regularity, we will prove the existence of quasi-periodic traveling waves $(\breve{\eta}, \breve{\psi})$ belonging to some Sobolev space

$$
H^{s}\left(\mathbb{T}^{v}, \mathbb{R}^{2}\right)=\left\{\breve{f}(\varphi)=\sum_{\ell \in \mathbb{Z}^{v}} f_{\ell} e^{\mathrm{i} \ell \cdot \varphi}, f_{\ell} \in \mathbb{R}^{2}:\|\breve{f}\|_{s}^{2}:=\sum_{\ell \in \mathbb{Z}^{v}}\left|f_{\ell}\right|^{2}\langle\ell\rangle^{2 s}<\infty\right\} .
$$

Fixed finitely many arbitrary distinct natural numbers

$$
\mathbb{S}^{+}:=\left\{\bar{n}_{1}, \ldots, \bar{n}_{v}\right\} \subset \mathbb{N}, \quad 1 \leqq \bar{n}_{1}<\ldots<\bar{n}_{v},
$$

and signs

$$
\Sigma:=\left\{\sigma_{1}, \ldots, \sigma_{\nu}\right\}, \quad \sigma_{a} \in\{-1,1\}, \quad a=1, \ldots, v
$$


consider the reversible quasi-periodic traveling wave solutions of the linear system (1.6) given by

$$
\begin{aligned}
\left(\begin{array}{l}
\eta(t, x) \\
\psi(t, x)
\end{array}\right) & =\sum_{a \in\left\{1, \ldots, \nu: \sigma_{a}=+1\right\}}\left(\begin{array}{c}
M_{\bar{n}_{a}} \sqrt{\xi_{\bar{n}_{a}}} \cos \left(\bar{n}_{a} x-\Omega_{\bar{n}_{a}}(\kappa) t\right) \\
P_{\bar{n}_{a}} \sqrt{\xi_{\bar{n}_{a}}} \sin \left(\bar{n}_{a} x-\Omega_{\bar{n}_{a}}(\kappa) t\right)
\end{array}\right) \\
& +\sum_{a \in\left\{1, \ldots, \nu: \sigma_{a}=-1\right\}}\left(\begin{array}{c}
M_{\bar{n}_{a}} \sqrt{\xi_{-\bar{n}_{a}}} \cos \left(\bar{n}_{a} x+\Omega_{-\bar{n}_{a}}(\kappa) t\right) \\
P_{-\bar{n}_{a}} \sqrt{\xi_{-\bar{n}_{a}}} \sin \left(\bar{n}_{a} x+\Omega_{-\bar{n}_{a}}(\kappa) t\right)
\end{array}\right),
\end{aligned}
$$

where $\xi_{ \pm \bar{n}_{a}}>0, a=1, \ldots, v$. The frequency vector of (1.17) is

$$
\vec{\Omega}(\kappa):=\left(\Omega_{\sigma_{a} \bar{n}_{a}}(\kappa)\right)_{a=1, \ldots, v} \in \mathbb{R}^{v} .
$$

Remark 1.4. If $\sigma_{a}=+1$, we select in (1.17) a right traveling wave, whereas, if $\sigma_{a}=-1$, a left traveling one. By (1.15), the linear solutions (1.17) are genuinely traveling waves: superposition of identical waves traveling in opposite direction, generating standing waves, does not happen.

The main result of this paper proves that the linear solutions (1.17) can be continued to quasi-periodic traveling wave solutions of the nonlinear water waves equations (1.3), for most values of the surface tension $\kappa \in\left[\kappa_{1}, \kappa_{2}\right]$, with a frequency vector $\widetilde{\Omega}:=\left(\widetilde{\Omega}_{\sigma_{a} \bar{n}_{a}}\right)_{a=1, \ldots, \nu}$, close to $\vec{\Omega}(\kappa):=\left(\Omega_{\sigma_{a} \bar{n}_{a}}(\kappa)\right)_{a=1, \ldots, \nu}$. Here is the precise statement:

Theorem 1.5. (KAM for traveling gravity-capillary water waves with constant vorticity) Consider finitely many tangential sites $\mathbb{S}^{+} \subset \mathbb{N}$ as in (1.15) and signs $\Sigma$ as in (1.16). Then there exist $\bar{s}>0, \varepsilon_{0} \in(0,1)$ such that, for every $|\xi| \leqq \varepsilon_{0}^{2}$, $\xi:=\left(\xi_{\sigma_{a} \bar{n}_{a}}\right)_{a=1, \ldots, \nu} \in \mathbb{R}_{+}^{v}$, the following hold:

1. there exists a Cantor-like set $\mathcal{G}_{\xi} \subset\left[\kappa_{1}, \kappa_{2}\right]$ with asymptotically full measure as $\xi \rightarrow 0$, that is $\lim _{\xi \rightarrow 0}\left|\mathcal{G}_{\xi}\right|=\kappa_{2}-\kappa_{1}$;

2. for any $\kappa \in \mathcal{G}_{\xi}$, the gravity-capillary water waves equations (1.3) have a reversible quasi-periodic traveling wave solution (according to Definition 1.2) of the form

$$
\begin{aligned}
& \left(\begin{array}{l}
\eta(t, x) \\
\psi(t, x)
\end{array}\right)=\sum_{a \in\{1, \ldots, \nu\}: \sigma_{a}=+1}\left(\begin{array}{c}
M_{\bar{n}_{a}} \sqrt{\xi_{\bar{n}_{a}}} \cos \left(\bar{n}_{a} x-\widetilde{\Omega}_{\bar{n}_{a}} t\right) \\
P_{\bar{n}_{a}} \sqrt{\xi_{\bar{n}_{a}}} \sin \left(\bar{n}_{a} x-\widetilde{\Omega}_{\bar{n}_{a}} t\right)
\end{array}\right) \\
& +\sum_{a \in\{1, \ldots, \nu\}: \sigma_{a}=-1}\left(\begin{array}{c}
M_{\bar{n}_{a}} \sqrt{\xi_{-\bar{n}_{a}}} \cos \left(\bar{n}_{a} x+\widetilde{\Omega}_{-\bar{n}_{a}} t\right) \\
P_{-\bar{n}_{a}} \sqrt{\xi_{-\bar{n}_{a}}} \sin \left(\bar{n}_{a} x+\widetilde{\Omega}_{-\bar{n}_{a}} t\right)
\end{array}\right)+r(t, x)
\end{aligned}
$$

where, for some $\breve{r} \in H^{\bar{s}}\left(\mathbb{T}^{v}, \mathbb{R}^{2}\right)$,

$$
r(t, x)=\breve{r}\left(\widetilde{\Omega}_{\sigma_{1} \bar{n}_{1}} t-\sigma_{1} \bar{n}_{1} x, \ldots, \widetilde{\Omega}_{\sigma_{v} \bar{n}_{v}} t-\sigma_{\nu} \bar{n}_{v} x\right), \quad \lim _{\xi \rightarrow 0} \frac{\|\breve{r}\|_{\bar{s}}}{\sqrt{|\xi|}}=0,
$$

with a Diophantine frequency vector $\widetilde{\Omega}:=\left(\widetilde{\Omega}_{\sigma_{a} \bar{n}_{a}}\right)_{a=1, \ldots, v} \in \mathbb{R}^{v}$, depending on $\kappa, \xi$, and satisfying $\lim _{\xi \rightarrow 0} \widetilde{\Omega}=\vec{\Omega}(\kappa)$. In addition these quasi-periodic solutions are linearly stable. 
Let us make some comments.

1) Theorem 1.5 holds for any value of the vorticity $\gamma$, so in particular it guarantees existence of quasi-periodic traveling waves also for irrotational fluids, that is $\gamma=0$. In this case the solutions (1.19) do not reduce to those in [6], which are standing, that is even in $x$. If the vorticity $\gamma \neq 0$, one does not expect the existence of standing wave solutions since the water waves vector field (1.3) does not leave invariant the subspace of functions even in $x$.

2) Theorem 1.5 produces time quasi-periodic solutions of the Euler equation with a velocity field which is a small perturbation of the Couette flow $\left(\begin{array}{c}-\gamma y \\ 0\end{array}\right)$. Indeed, from the solution $(\eta(t, x), \psi(t, x))$ in (1.19), one recovers the generalized velocity potential $\Phi(t, x, y)$ by solving the elliptic problem (1.2) and finally constructs the velocity field $\left(\begin{array}{l}u(t, x, y) \\ v(t, x, y)\end{array}\right)=\left(\begin{array}{c}-\gamma y \\ 0\end{array}\right)+\nabla \Phi(t, x, y)$. The time quasi-periodic potential $\Phi(t, x, y)$ has size $O(\sqrt{|\xi|})$, as $\eta(t, x)$ and $\psi(t, x)$.

3 ) In the case $v=1$ the solutions constructed in Theorem 1.5 reduce to steady periodic traveling waves, which can be obtained by an application of the CrandallRabinowitz theorem, see for example [30,41,43].

4) Theorem 1.5 selects initial data giving raise to global in time solutions (1.19) of the water waves equations (1.3). So far, no results about global existence for (1.3) with periodic boundary conditions are known. The available results concern local well posedness with a general vorticity, see for example [10], and a $\varepsilon^{-2}$ existence for initial data of size $\varepsilon$ in the case of constant vorticity [21].

5) With the choice (1.15)-(1.16) the unperturbed frequency vector $\vec{\Omega}(\kappa)=$ $\left(\Omega_{\sigma_{a} \bar{n}_{a}}(\kappa)\right)_{a=1, \ldots, \nu}$ is diophantine for most values of the surface tension $\kappa$ and for all values of vorticity, gravity and depth. It follows by the more general results of Sections 4 and 5.2. This may not be true for an arbitrary choice of the linear frequencies $\Omega_{j}(\kappa), j \in \mathbb{Z} \backslash\{0\}$. For example, in the case $\mathrm{h}=+\infty$, the vector

$$
\vec{\Omega}(\kappa)=\left(\Omega_{-n_{3}}(\kappa), \Omega_{-n_{2}}(\kappa), \Omega_{-n_{1}}(\kappa), \Omega_{n_{1}}(\kappa), \Omega_{n_{2}}(\kappa), \Omega_{n_{3}}(\kappa)\right)
$$

is resonant, for all the values of $\kappa$, also taking into account the restrictions on the indexes for the search of traveling waves, see Section 3.4. Indeed, recalling (1.11) and that, for $\mathrm{h}=+\infty, G_{j}(0, \mathrm{~h})=|j|$, we have, for $\ell=$ $\left(-\ell_{n_{3}},-\ell_{n_{2}},-\ell_{n_{1}}, \ell_{n_{1}}, \ell_{n_{2}}, \ell_{n_{3}}\right)$ that the system

$$
\vec{\Omega}(\kappa) \cdot \vec{\ell}=\gamma\left(\ell_{n_{1}}+\ell_{n_{2}}+\ell_{n_{3}}\right)=0, \quad n_{1} \ell_{n_{1}}+n_{2} \ell_{n_{2}}+n_{3} \ell_{n_{3}}=0,
$$

has integer solutions. In this case the possible existence of quasi-periodic solutions of the water waves system (1.3) depends on the frequency modulation induced by the nonlinear terms.

6) Comparison with [6]. There are significant differences with respect to [6], which proves the existence of quasi-periodic standing waves for irrotational fluids, not only in the result - the solutions of Theorem 1.5 are traveling waves of fluids with constant vorticity - but also in the techniques. 
(1) The first difference -which is a novelty of this paper-is a new formulation of degenerate KAM theory exploiting the "momentum conservation", namely the space invariance of the Hamilton equations. The degenerate KAM theory approach for PDEs has been developed in [3], and then [2,6], in order to prove the non-trivial dependence of the linear frequencies with respect to a parameter -in our case the surface tension $\kappa-$, see the "Transversality" Proposition 4.5. A key assumption used in $[2,3,6]$ is that the linear frequencies are simple (because of Dirichlet boundary conditions in [3] and Neumann boundary conditions in $[2,6]$ ). This is not true for traveling waves (for example in case of zero vorticity one has $\Omega_{j}(\kappa)=\Omega_{-j}(\kappa)$ identically in $\left.\kappa\right)$. In order to deal with these resonances we strongly exploit the invariance of the equations (1.3) under space translations, which ultimately imply the restrictions to the indexes (4.8)-(4.10). In this way, assuming that the moduli of the tangential sites are all different as in (1.15), cfr. with item 5), we can remove some otherwise possibly degenerate case. This requires us to keep track along all of the proof of the "momentum conservation property" that we characterize in different ways in Section 3.4. The momentum conservation law has been used in several KAM results for semilinear PDEs since the works [16, 17, 28,35]; see also $[15,20,31]$ and references therein. The present paper gives a new application in the context of degenerate KAM theory (with additional difficulties arising by the quasi-linear nature of the water waves equations).

(2) Other significant differences with respect to [6] arise in the reduction in orders (Section 7) of the quasi-periodic linear operators obtained along the Nash-Moser iteration. In particular, we mention that we have to preserve the Hamiltonian nature of these operators (at least until Section 7.4). Otherwise it would appear a time dependent operator at the order $|D|^{1 / 2}$, of the form i $a(\varphi) \mathcal{H}|D|^{\frac{1}{2}}$, with $a(\varphi) \in \mathbb{R}$ independent of $x$, compatible with the reversible structure, which can not be eliminated. Note that the operator $i a(\varphi) \mathcal{H}|D|^{\frac{1}{2}}$ is not Hamiltonian (unless $a(\varphi)=0$ ). Note also that the above difficulty was not present in [6] dealing with standing waves, because an operator of the form i $a(\varphi) \mathcal{H}|D|^{\frac{1}{2}}$ does not map even functions into even functions. In order to overcome this difficulty we have to perform always symplectic changes of variables (at least until Section 7.4), and not just reversible ones as in [2,6]. We finally mention that we perform as a first step in Section 7.1 a quasiperiodic time reparametrization to avoid otherwise a technical difficulty in the conjugation of the remainders obtained by the Egorov theorem in Section 7.3. This difficulty was not present in [6], since it arises conjugating the additional pseudodifferential term due to vorticity, see Remark 7.5.

7) Another novelty of our result is to exploit the momentum conservation also to prove that the obtained quasi-periodic solutions are indeed quasi-periodic traveling waves, according to Definition 1.2. This requires checking that the approximate solutions constructed along the Nash-Moser iteration of Section 9 (and Section 6) are indeed traveling waves. Actually this approach shows that the preservation of the momentum condition along the Nash-Moser-KAM iteration is equivalent to the construction of embedded invariant tori which sup- 
port quasi-periodic traveling waves, namely of the form $u(\varphi, x)=U(\varphi-\vec{\jmath} x)$ (see Definition 3.1), or equivalently, in action-angle-normal variables, which satisfy (3.52). We expect that this method can be used to obtain quasi-periodic traveling waves for other PDE's which are translation invariant.

Literature. We now shortly describe the literature regarding the existence of time periodic or quasi-periodic solutions of the water waves equations, focusing on the results more related to Theorem 1.5. We describes only results concerning space periodic waves, that we divide in three distinct groups:

(i) steady traveling solutions,

(ii) time periodic standing waves,

(iii) time quasi-periodic standing waves.

This distinction takes into account not only the different shapes of the waves, but also the techniques for their construction.

(i) Time and space periodic traveling waves which are steady in a moving frame. The literature concerning steady traveling wave solutions is huge, and we refer to [7] for an extended presentation. Here we only mention that, after the pioneering work of STOKEs [38], the first rigorous construction of small amplitude space periodic steady traveling waves goes back to the 1920s with the papers of NEKRAsov [33], LEVI-CiVITA [27] and STRUIK [39], in case of irrotational bidimensional flows under the action of pure gravity. Later ZEIDLER [47] considered the effect of capillarity. In the presence of vorticity, the first result is due to GERSTNER [18] in 1802, who gave an explicit example of periodic traveling wave, in infinite depth, and with a particular non-zero vorticity. One has to await the work of DUBREIL-JACOTIN [14] in 1934 for the first existence results of small amplitude, periodic traveling waves with general (Hölder continuous, small) vorticity, and, later, the works of GoYON [19] and ZEIDLER [48] in the case of large vorticity. More recently we point out the works of WAHLÉN [41] for capillary-gravity waves and non-constant vorticity, and of MARTIN [30] and WALHÉn [42] for constant vorticity. All these results deal with $2 \mathrm{~d}$ water waves and can ultimately be deduced by the Crandall-Rabinowitz bifurcation theorem from a simple eigenvalue.

We also mention that these local bifurcation results can be extended to global branches of steady traveling waves by applying the methods of global bifurcation theory. We refer to Keady-Norbury [29], Toland [40], McLeod [32] for irrotational flows and CONSTANTIN-STRAUSs [9] for fluids with non-constant vorticity.

In the case of three dimensional irrotational fluids, bifurcation of small amplitude traveling waves periodic in space has been proved in REEDER-SHINBRot [36], CRAIG-NICHOLLS $[11,12]$ for both gravity-capillary waves (by variational bifurcation arguments a la Weinstein-Moser) and by Iooss-PLOTNIKOV [23,24] for gravity waves (this is a small divisor problem). These solutions, in a moving frame, look steady bi-periodic waves.

(ii) Time periodic standing waves. Bifurcation of time periodic standing water waves were obtained in a series of pioneering papers by Iooss, PlotNIKOV and TolAnd [22,23, 25,34] for pure gravity waves, and by AlAzARD-BALDI [1] for gravity-capillary fluids. Standing waves are even in the space variable and so they 
do not travel in space. There is a huge difference with the results of the first group: the construction of time periodic standing waves involves small divisors. Thus the proof is based on Nash-Moser implicit function techniques and not only on the classical implicit function theorem.

(iii) Time quasi-periodic standing waves. The first results in this direction were obtained very recently by BERTI-MONTALTO [6] for the gravity-capillary system and by BALDI-BERTI-HAUS-MonTALTO [2] for the gravity water waves. Both papers deal with irrotational fluids.

We finally mention the very recent numerical works of WILKENING-ZHAO [44, 45] about spatially quasi-periodic gravity-capillary 1d-water waves. In particular, the analysis in [45] is complementary to Theorem 1.5; the solutions (1.19) are time-quasi-periodic traveling waves on a spatially periodic domain, whereas [45] concerns pure traveling waves with multiple spatial periods.

\section{Hamiltonian Structure and Linearization at the Origin}

In this section we describe the Hamiltonian structure of the water waves equations (1.3), their symmetries and the solutions of the linearized system (1.6) at the equilibrium.

\subsection{Hamiltonian Structure}

The Hamiltonian formulation of the water waves equations (1.3) with nonzero constant vorticity was obtained by CONSTANTIN-IVANOV-ProdANOV [8] and WAHLÉN [42] in the case of finite depth. For irrotational flows it reduces to the classical Craig-Sulem-ZaKHAROV formulation in $[13,46]$.

On the phase space $H_{0}^{1}(\mathbb{T}) \times \dot{H}^{1}(\mathbb{T})$, endowed with the non canonical Poisson tensor

$$
J_{M}(\gamma):=\left(\begin{array}{cc}
0 & \mathrm{Id} \\
-\mathrm{Id} & \gamma \partial_{x}^{-1}
\end{array}\right),
$$

we consider the Hamiltonian

$$
H(\eta, \psi)=\frac{1}{2} \int_{\mathbb{T}}\left(\psi G(\eta) \psi+g \eta^{2}\right) \mathrm{d} x+\kappa \int_{\mathbb{T}} \sqrt{1+\eta_{x}^{2}} \mathrm{~d} x+\frac{\gamma}{2} \int_{\mathbb{T}}\left(-\psi_{x} \eta^{2}+\frac{\gamma}{3} \eta^{3}\right) \mathrm{d} x .
$$

Such Hamiltonian is well defined on $H_{0}^{1}(\mathbb{T}) \times \dot{H}^{1}(\mathbb{T})$ since $G(\eta)[1]=0$ and $\int_{\mathbb{T}} G(\eta) \psi \mathrm{d} x=0$.

It turns out $[8,42]$ that equations $(1.3)$ are the Hamiltonian system generated by $H(\eta, \psi)$ with respect to the Poisson tensor $J_{M}(\gamma)$, namely

$$
\partial_{t}\left(\begin{array}{c}
\eta \\
\psi
\end{array}\right)=J_{M}(\gamma)\left(\begin{array}{c}
\nabla_{\eta} H \\
\nabla_{\psi} H
\end{array}\right),
$$

where $\left(\nabla_{\eta} H, \nabla_{\psi} H\right) \in \dot{L}^{2}(\mathbb{T}) \times L_{0}^{2}(\mathbb{T})$ denote the $L^{2}$-gradients. 
Remark 2.1. The non canonical Poisson tensor $J_{M}(\gamma)$ in (2.1) has to be regarded as an operator from (subspaces of) $\left(L_{0}^{2} \times \dot{L}^{2}\right)^{*}=\dot{L}^{2} \times L_{0}^{2}$ to $L_{0}^{2} \times \dot{L}^{2}$, that is

$$
J_{M}(\gamma)=\left(\begin{array}{cc}
0 & \operatorname{Id}_{L_{0}^{2} \rightarrow L_{0}^{2}} \\
-\operatorname{Id}_{\dot{L}^{2} \rightarrow \dot{L}^{2}} & \gamma \partial_{x}^{-1}
\end{array}\right)
$$

The operator $\partial_{x}^{-1}$ maps a dense subspace of $L_{0}^{2}$ in $\dot{L}^{2}$. For sake of simplicity, throughout the paper we may omit this detail. Above the dual space $\left(L_{0}^{2} \times \dot{L}^{2}\right)^{*}$ with respect to the scalar product in $L^{2}$ is identified with $\dot{L}^{2} \times L_{0}^{2}$.

The Hamiltonian (2.2) enjoys several symmetries which we now describe.

Reversible structure. Defining on the phase space $H_{0}^{1}(\mathbb{T}) \times \dot{H}^{1}(\mathbb{T})$ the involution

$$
\mathcal{S}\left(\begin{array}{c}
\eta \\
\psi
\end{array}\right):=\left(\begin{array}{c}
\eta^{\vee} \\
-\psi^{\vee}
\end{array}\right), \quad \eta^{\vee}(x):=\eta(-x),
$$

the Hamiltonian (2.2) is invariant under $\mathcal{S}$; that is

$$
H \circ \mathcal{S}=H
$$

or, equivalently, the water waves vector field $X$ defined in the right hand side on (1.3) satisfies

$$
X \circ \mathcal{S}=-\mathcal{S} \circ X
$$

This property follows, noting that the Dirichlet-Neumann operator satisfies

$$
G\left(\eta^{\vee}\right)\left[\psi^{\vee}\right]=(G(\eta)[\psi])^{\vee}
$$

Translation invariance. Since the bottom of the fluid domain (1.1) is flat (or in case of infinite depth there is no bottom), the water waves equations (1.3) are invariant under space translations. Specifically, defining the translation operator

$$
\tau_{\varsigma}: u(x) \mapsto u(x+\varsigma), \quad \varsigma \in \mathbb{R}
$$

the Hamiltonian (2.2) satisfies $H \circ \tau_{\varsigma}=H$ for any $\varsigma \in \mathbb{R}$, or, equivalently, the water waves vector field $X$ defined in the right hand side on (1.3) satisfies

$$
X \circ \tau_{\varsigma}=\tau_{\varsigma} \circ X, \quad \forall \varsigma \in \mathbb{R} .
$$

In order to verify this property, note that the Dirichlet-Neumann operator satisfies

$$
\tau_{\varsigma} \circ G(\eta)=G\left(\tau_{\varsigma} \eta\right) \circ \tau_{\varsigma}, \quad \forall \varsigma \in \mathbb{R}
$$


Wahlén coordinates. The variables $(\eta, \psi)$ are not Darboux coordinates, in the sense that the Poisson tensor (2.1) is not the canonical one for values of the vorticity $\gamma \neq 0$. WAHLÉN [42] noted that in the variables $(\eta, \zeta)$, where $\zeta$ is defined by

$$
\zeta:=\psi-\frac{\gamma}{2} \partial_{x}^{-1} \eta
$$

the symplectic form induced by $J_{M}(\gamma)$ becomes the canonical one. Indeed, under the linear transformation of the phase space $H_{0}^{1} \times \dot{H}^{1}$ into itself defined by

$$
\left(\begin{array}{c}
\eta \\
\psi
\end{array}\right)=W\left(\begin{array}{l}
\eta \\
\zeta
\end{array}\right), \quad W:=\left(\begin{array}{cc}
\mathrm{Id} & 0 \\
\frac{\gamma}{2} \partial_{x}^{-1} & \mathrm{Id}
\end{array}\right), \quad W^{-1}:=\left(\begin{array}{cc}
\mathrm{Id} & 0 \\
-\frac{\gamma}{2} \partial_{x}^{-1} & \mathrm{Id}
\end{array}\right),
$$

the Poisson tensor $J_{M}(\gamma)$ is transformed into the canonical one,

$$
W^{-1} J_{M}(\gamma)\left(W^{-1}\right)^{*}=J, \quad J:=\left(\begin{array}{cc}
0 & \text { Id } \\
- \text { Id } & 0
\end{array}\right) .
$$

Here $W^{*}$ and $\left(W^{-1}\right)^{*}$ are the adjoints maps from (a dense subspace of) $\dot{L}^{2} \times L_{0}^{2}$ into itself, and the Poisson tensor $J$ acts from (subspaces of) $\dot{L}^{2} \times L_{0}^{2}$ to $L_{0}^{2} \times \dot{L}^{2}$. Then the Hamiltonian (2.2) becomes

$$
\mathcal{H}:=H \circ W, \quad \text { that is } \mathcal{H}(\eta, \zeta):=H\left(\eta, \zeta+\frac{\gamma}{2} \partial_{x}^{-1} \eta\right),
$$

and the Hamiltonian equations (2.3) (that is (1.3)) are transformed into

$$
\partial_{t}\left(\begin{array}{l}
\eta \\
\zeta
\end{array}\right)=X_{\mathcal{H}}(\eta, \zeta), \quad X_{\mathcal{H}}(\eta, \zeta):=J\left(\begin{array}{l}
\nabla_{\eta} \mathcal{H} \\
\nabla_{\zeta} \mathcal{H}
\end{array}\right)(\eta, \zeta)
$$

By (2.12), the symplectic form of (2.14) is the standard one,

$$
\mathcal{W}\left(\left(\begin{array}{l}
\eta_{1} \\
\zeta_{1}
\end{array}\right),\left(\begin{array}{l}
\eta_{2} \\
\zeta_{2}
\end{array}\right)\right)=\left(J^{-1}\left(\begin{array}{l}
\eta_{1} \\
\zeta_{1}
\end{array}\right),\left(\begin{array}{l}
\eta_{2} \\
\zeta_{2}
\end{array}\right)\right)_{L^{2}}=\left(-\zeta_{1}, \eta_{2}\right)_{L^{2}}+\left(\eta_{1}, \zeta_{2}\right)_{L^{2}}
$$

where $J^{-1}$ is the symplectic operator

$$
J^{-1}=\left(\begin{array}{cc}
0 & -\mathrm{Id} \\
\mathrm{Id} & 0
\end{array}\right)
$$

regarded as a map from $L_{0}^{2} \times \dot{L}^{2}$ into $\dot{L}^{2} \times L_{0}^{2}$. Note that $J J^{-1}=\operatorname{Id}_{L_{0}^{2} \times \dot{L}^{2}}$ and $J^{-1} J=\operatorname{Id}_{L^{2} \times L_{0}^{2}}$. The Hamiltonian vector field $X_{\mathcal{H}}(\eta, \zeta)$ in (2.14) is characterized by the identity

$$
d \mathcal{H}(\eta, \zeta)[\widehat{u}]=\mathcal{W}\left(X_{\mathcal{H}}(\eta, \zeta), \widehat{u}\right), \quad \forall \widehat{u}:=\left(\frac{\widehat{\eta}}{\widehat{\zeta}}\right) .
$$

The transformation $W$ defined in (2.11) is reversibility preserving, namely it commutes with the involution $\mathcal{S}$ in (2.4) (see Definition 3.17 below), and thus also the Hamiltonian $\mathcal{H}$ in (2.13) is invariant under the involution $\mathcal{S}$, as well as $H$ in 
(2.2). For this reason we look for solutions $(\eta(t, x), \zeta(t, x))$ of (2.14) which are reversible, that is see (1.5),

$$
\left(\begin{array}{l}
\eta \\
\zeta
\end{array}\right)(-t)=\mathcal{S}\left(\begin{array}{l}
\eta \\
\zeta
\end{array}\right)(t)
$$

The corresponding solutions $(\eta(t, x), \psi(t, x))$ of (1.3) induced by (2.11) are reversible as well.

We finally note that the transformation $W$ defined in (2.11) commutes with the translation operator $\tau_{\zeta}$, therefore the Hamiltonian $\mathcal{H}$ in (2.13) is invariant under $\tau_{\varsigma}$, as well as $H$ in (2.2). By Noether theorem, the horizontal momentum $\int_{\mathbb{T}} \zeta \eta_{x} \mathrm{~d} x$ is a prime integral of (2.14).

\subsection{Linearization at the Equilibrium}

In this section we study the linear system (1.6) and prove that its reversible solutions have the form (1.9).

In view of the Hamiltonian (2.2) of the water waves equations (1.3), also the linear system (1.6) is Hamiltonian and it is generated by the quadratic Hamiltonian

$$
H_{L}(\eta, \psi):=\frac{1}{2} \int_{\mathbb{T}}\left(\psi G(0) \psi+g \eta^{2}+\kappa \eta_{x}^{2}\right) \mathrm{d} x=\frac{1}{2}\left(\boldsymbol{\Omega}_{L}\left(\begin{array}{c}
\eta \\
\psi
\end{array}\right),\left(\begin{array}{c}
\eta \\
\psi
\end{array}\right)\right)_{L^{2}} .
$$

Thus, recalling (2.3), the linear system (1.6) is

$$
\partial_{t}\left(\begin{array}{c}
\eta \\
\psi
\end{array}\right)=J_{M}(\gamma) \boldsymbol{\Omega}_{L}\left(\begin{array}{c}
\eta \\
\psi
\end{array}\right), \quad \boldsymbol{\Omega}_{L}:=\left(\begin{array}{cc}
-\kappa \partial_{x}^{2}+g & 0 \\
0 & G(0)
\end{array}\right) .
$$

The linear operator $\boldsymbol{\Omega}_{L}$ acts from (a dense subspace) of $L_{0}^{2} \times \dot{L}^{2}$ to $\dot{L}^{2} \times L_{0}^{2}$. In the Wahlén coordinates (2.11), the linear Hamiltonian system (1.6), that is (2.18), transforms into the linear Hamiltonian system

$$
\begin{aligned}
& \partial_{t}\left(\begin{array}{l}
\eta \\
\zeta
\end{array}\right)=J \boldsymbol{\Omega}_{W}\left(\begin{array}{l}
\eta \\
\zeta
\end{array}\right), \\
& \boldsymbol{\Omega}_{W}:=W^{*} \boldsymbol{\Omega}_{L} W=\left(\begin{array}{cc}
-\kappa \partial_{x}^{2}+g-\left(\frac{\gamma}{2}\right)^{2} \partial_{x}^{-1} G(0) \partial_{x}^{-1} & -\frac{\gamma}{2} \partial_{x}^{-1} G(0) \\
\frac{\gamma}{2} G(0) \partial_{x}^{-1} & G(0)
\end{array}\right)
\end{aligned}
$$

generated by the quadratic Hamiltonian

$$
\mathcal{H}_{L}(\eta, \zeta):=\left(H_{L} \circ W\right)(\eta, \zeta)=\frac{1}{2}\left(\boldsymbol{\Omega}_{W}\left(\begin{array}{l}
\eta \\
\zeta
\end{array}\right),\left(\begin{array}{l}
\eta \\
\zeta
\end{array}\right)\right)_{L^{2}}
$$

The linear operator $\boldsymbol{\Omega}_{W}$ acts from (a dense subspace) of $L_{0}^{2} \times \dot{L}^{2}$ to $\dot{L}^{2} \times L_{0}^{2}$. The linear system (2.19) is the Hamiltonian system obtained by linearizing (2.14) at the equilibrium $(\eta, \zeta)=(0,0)$. We want to transform (2.19) in diagonal form by using a symmetrizer and then introducing complex coordinates. We first conjugate (2.19) 
under the symplectic transformation (with respect to the standard symplectic form $\mathcal{W}$ in (2.15)) of the phase space

$$
\left(\begin{array}{l}
\eta \\
\zeta
\end{array}\right)=\mathcal{M}\left(\begin{array}{l}
u \\
v
\end{array}\right)
$$

where $\mathcal{M}$ is the diagonal matrix of self-adjoint Fourier multipliers

$$
\mathcal{M}:=\left(\begin{array}{cc}
M(D) & 0 \\
0 & M(D)^{-1}
\end{array}\right), M(D):=\left(\frac{G(0)}{\kappa D^{2}+g-\frac{\gamma^{2}}{4} \partial_{x}^{-1} G(0) \partial_{x}^{-1}}\right)^{1 / 4},
$$

with the real valued symbol $M_{j}$ defined in (1.10). The map $\mathcal{M}$ is reversibility preserving.

Remark 2.2. In (2.21) the Fourier multiplier $M(D)$ acts in $H_{0}^{1}$. On the other hand, with a slight abuse of notation, $M(D)^{-1}$ denotes the Fourier multiplier operator in $\dot{H}^{1}$ defined as

$$
M(D)^{-1}[\zeta]:=\left[\sum_{j \neq 0} M_{j}^{-1} \zeta_{j} e^{i j x}\right], \quad \zeta(x)=\sum_{j \in \mathbb{Z}} \zeta_{j} e^{i j x}
$$

where $[\zeta]$ is the element in $\dot{H}^{1}$ with representant $\zeta(x)$.

By a direct computation, the Hamiltonian system (2.19) assumes the symmetric form

$\partial_{t}\left(\begin{array}{l}u \\ v\end{array}\right)=J \boldsymbol{\Omega}_{S}\left(\begin{array}{l}u \\ v\end{array}\right), \quad \boldsymbol{\Omega}_{S}:=\mathcal{M}^{*} \boldsymbol{\Omega}_{W} \mathcal{M}=\left(\begin{array}{cc}\omega(\kappa, D) & -\frac{\gamma}{2} \partial_{x}^{-1} G(0) \\ \frac{\gamma}{2} G(0) \partial_{x}^{-1} & \omega(\kappa, D)\end{array}\right)$,

where

$$
\omega(\kappa, D):=\sqrt{\kappa D^{2} G(0)+g G(0)-\left(\frac{\gamma}{2} \partial_{x}^{-1} G(0)\right)^{2}}
$$

Remark 2.3. To be precise, the Fourier multiplier operator $\omega(\kappa, D)$ in the top left position in (2.22) maps $H_{0}^{1}$ into $\dot{H}^{1}$ and the one in the bottom right position maps $\dot{H}^{1}$ into $H_{0}^{1}$. The operator $\partial_{x}^{-1} G(0)$ acts on $\dot{H}^{1}$ and $G(0) \partial_{x}^{-1}$ on $H_{0}^{1}$.

Now we introduce complex coordinates by the transformation

$$
\left(\begin{array}{l}
u \\
v
\end{array}\right)=\mathcal{C}\left(\begin{array}{l}
z \\
z
\end{array}\right), \quad \mathcal{C}:=\frac{1}{\sqrt{2}}\left(\begin{array}{cc}
\text { Id Id } \\
-\mathrm{i} & \mathrm{i}
\end{array}\right), \quad \mathcal{C}^{-1}:=\frac{1}{\sqrt{2}}\left(\begin{array}{cc}
\mathrm{Id} & \mathrm{i} \\
\mathrm{Id} & -\mathrm{i}
\end{array}\right) .
$$

In these variables, the Hamiltonian system (2.22) becomes the diagonal system

$\partial_{t}\left(\begin{array}{c}z \\ \bar{z}\end{array}\right)=\left(\begin{array}{cc}-\mathrm{i} & 0 \\ 0 & \mathrm{i}\end{array}\right) \boldsymbol{\Omega}_{D}\left(\begin{array}{c}z \\ \bar{z}\end{array}\right), \quad \boldsymbol{\Omega}_{D}:=\mathcal{C}^{*} \boldsymbol{\Omega}_{S} \mathcal{C}=\left(\begin{array}{cc}\Omega(\kappa, D) & 0 \\ 0 & \bar{\Omega}(\kappa, D)\end{array}\right)$,

where

$$
\Omega(\kappa, D):=\omega(\kappa, D)+\mathrm{i} \frac{\gamma}{2} \partial_{x}^{-1} G(0)
$$


is the Fourier multiplier with symbol $\Omega_{j}(\kappa)$ defined in $(1.11)$ and $\bar{\Omega}(\kappa, D)$ is defined by

$$
\bar{\Omega}(\kappa, D) z:=\overline{\Omega(\kappa, D) \bar{z}}, \quad \bar{\Omega}(\kappa, D)=\omega(\kappa, D)-\mathrm{i} \frac{\gamma}{2} \partial_{x}^{-1} G(0) .
$$

Note that $\bar{\Omega}(\kappa, D)$ is the Fourier multiplier with symbol $\left\{\Omega_{-j}(\kappa)\right\}_{j \in \mathbb{Z} \backslash\{0\}}$.

Remark 2.4. We regard the system (2.25) in $\dot{H}^{1} \times \dot{H}^{1}$.

The diagonal system (2.25) amounts to the scalar equation

$$
\partial_{t} z=-\mathrm{i} \Omega(\kappa, D) z, \quad z(x)=\sum_{j \in \mathbb{Z} \backslash\{0\}} z_{j} e^{\mathrm{i} j x},
$$

and, writing (2.27) in the exponential Fourier basis, to the infinitely many decoupled harmonic oscillators

$$
\dot{z}_{j}=-\mathrm{i} \Omega_{j}(\kappa) z_{j}, \quad j \in \mathbb{Z} \backslash\{0\} .
$$

Note that, in these complex coordinates, the involution $\mathcal{S}$ defined in (2.4) reads as the map

$$
\left(\frac{z(x)}{z(x)}\right) \mapsto\left(\begin{array}{c}
\overline{z(-x)} \\
z(-x)
\end{array}\right),
$$

which we may read just as the scalar map $z(x) \mapsto \overline{z(-x)}$. Moreover, in the Fourier coordinates introduced in (2.27), it amounts to

$$
z_{j} \mapsto \overline{z_{j}}, \quad \forall j \in \mathbb{Z} \backslash\{0\} .
$$

In view of (2.28) and (2.30) every reversible solution (which is characterized as in (2.17)) of (2.27) has the form

$$
z(t, x):=\frac{1}{\sqrt{2}} \sum_{j \in \mathbb{Z} \backslash\{0\}} \rho_{j} e^{-\mathrm{i}\left(\Omega_{j}(\kappa) t-j x\right)} \quad \text { with } \rho_{j} \in \mathbb{R} .
$$

Let us see the form of these solutions back in the original variables $(\eta, \psi)$. First, by $(2.21),(2.24)$,

$$
\begin{aligned}
\left(\begin{array}{l}
\eta \\
\zeta
\end{array}\right) & =\mathcal{M C}\left(\begin{array}{l}
z \\
\bar{z}
\end{array}\right)=\frac{1}{\sqrt{2}}\left(\begin{array}{cc}
M(D) & M(D) \\
-\mathrm{i} M(D)^{-1} \mathrm{i} M(D)^{-1}
\end{array}\right)\left(\begin{array}{l}
z \\
\bar{z}
\end{array}\right) \\
& =\frac{1}{\sqrt{2}}\left(\begin{array}{c}
M(D)(z+\bar{z}) \\
-\mathrm{i} M(D)^{-1}(z-\bar{z})
\end{array}\right),
\end{aligned}
$$

and the solutions (2.31) assume the form

$$
\begin{aligned}
\left(\begin{array}{l}
\eta(t, x) \\
\zeta(t, x)
\end{array}\right) & =\sum_{n \in \mathbb{N}}\left(\begin{array}{c}
M_{n} \rho_{n} \cos \left(n x-\Omega_{n}(\kappa) t\right) \\
M_{n}^{-1} \rho_{n} \sin \left(n x-\Omega_{n}(\kappa) t\right)
\end{array}\right) \\
& +\sum_{n \in \mathbb{N}}\left(\begin{array}{c}
M_{n} \rho_{-n} \cos \left(n x+\Omega_{-n}(\kappa) t\right) \\
-M_{n}^{-1} \rho_{-n} \sin \left(n x+\Omega_{-n}(\kappa) t\right)
\end{array}\right) .
\end{aligned}
$$

Back to the variables $(\eta, \psi)$ with the change of coordinates (2.11) one obtains formula (1.9). 
Decomposition of the phase space in Lagrangian subspaces invariant under (2.19). We express the Fourier coefficients $z_{j} \in \mathbb{C}$ in (2.27) as

$$
z_{j}=\frac{\alpha_{j}+\mathrm{i} \beta_{j}}{\sqrt{2}}, \quad\left(\alpha_{j}, \beta_{j}\right) \in \mathbb{R}^{2}, \quad j \in \mathbb{Z} \backslash\{0\} .
$$

In the new coordinates $\left(\alpha_{j}, \beta_{j}\right)_{j \in \mathbb{Z} \backslash\{0\}}$, we write (2.32) as (recall that $M_{j}=M_{-j}$ )

$$
\left(\begin{array}{l}
\eta(x) \\
\zeta(x)
\end{array}\right)=\sum_{j \in \mathbb{Z} \backslash\{0\}}\left(\begin{array}{c}
M_{j}\left(\alpha_{j} \cos (j x)-\beta_{j} \sin (j x)\right) \\
M_{j}^{-1}\left(\beta_{j} \cos (j x)+\alpha_{j} \sin (j x)\right)
\end{array}\right)
$$

with

$$
\begin{aligned}
\alpha_{j} & =\frac{1}{2 \pi}\left(M_{j}^{-1}(\eta, \cos (j x))_{L^{2}}+M_{j}(\zeta, \sin (j x))_{L^{2}}\right), \\
\beta_{j} & =\frac{1}{2 \pi}\left(M_{j}(\zeta, \cos (j x))_{L^{2}}-M_{j}^{-1}(\eta, \sin (j x))_{L^{2}}\right) .
\end{aligned}
$$

The symplectic form (2.15) then becomes

$$
2 \pi \sum_{j \in \mathbb{Z} \backslash\{0\}} \mathrm{d} \alpha_{j} \wedge \mathrm{d} \beta_{j} .
$$

Each 2-dimensional subspace in the sum (2.33), spanned by $\left(\alpha_{j}, \beta_{j}\right) \in \mathbb{R}^{2}$ is therefore a symplectic subspace. The quadratic Hamiltonian $\mathcal{H}_{L}$ in (2.20) reads as

$$
2 \pi \sum_{j \in \mathbb{Z} \backslash\{0\}} \frac{\Omega_{j}(\kappa)}{2}\left(\alpha_{j}^{2}+\beta_{j}^{2}\right) .
$$

In view of (2.33), the involution $\mathcal{S}$ defined in (2.4) reads as

$$
\left(\alpha_{j}, \beta_{j}\right) \mapsto\left(\alpha_{j},-\beta_{j}\right), \quad \forall j \in \mathbb{Z} \backslash\{0\},
$$

and the translation operator $\tau_{\varsigma}$ defined in (2.7) as

$$
\left(\begin{array}{c}
\alpha_{j} \\
\beta_{j}
\end{array}\right) \mapsto\left(\begin{array}{cc}
\cos (j \varsigma) & -\sin (j \varsigma) \\
\sin (j \varsigma) & \cos (j \varsigma)
\end{array}\right)\left(\begin{array}{c}
\alpha_{j} \\
\beta_{j}
\end{array}\right), \quad \forall j \in \mathbb{Z} \backslash\{0\}
$$

We may also enumerate the independent variables $\left(\alpha_{j}, \beta_{j}\right)_{j \in \mathbb{Z} \backslash\{0\}}$ as $\left(\alpha_{-n}, \beta_{-n}\right.$, $\left.\alpha_{n}, \beta_{n}\right), n \in \mathbb{N}$. Thus the phase space $\mathfrak{H}:=L_{0}^{2} \times \dot{L}^{2}$ of (2.14) decomposes as the direct sum

$$
\mathfrak{H}=\sum_{n \in \mathbb{N}} V_{n,+} \oplus V_{n,-}
$$

of 2-dimensional Lagrangian symplectic subspaces

$$
V_{n,+}:=\left\{\left(\begin{array}{l}
\eta \\
\zeta
\end{array}\right)=\left(\begin{array}{c}
M_{n}\left(\alpha_{n} \cos (n x)-\beta_{n} \sin (n x)\right) \\
M_{n}^{-1}\left(\beta_{n} \cos (n x)+\alpha_{n} \sin (n x)\right)
\end{array}\right),\left(\alpha_{n}, \beta_{n}\right) \in \mathbb{R}^{2}\right\},
$$




$$
V_{n,-}:=\left\{\left(\begin{array}{l}
\eta \\
\zeta
\end{array}\right)=\left(\begin{array}{c}
M_{n}\left(\alpha_{-n} \cos (n x)+\beta_{-n} \sin (n x)\right) \\
M_{n}^{-1}\left(\beta_{-n} \cos (n x)-\alpha_{-n} \sin (n x)\right)
\end{array}\right),\left(\alpha_{-n}, \beta_{-n}\right) \in \mathbb{R}^{2}\right\},
$$

which are invariant for the linear Hamiltonian system (2.19), namely $J \boldsymbol{\Omega}_{W}$ : $V_{n, \sigma} \mapsto V_{n, \sigma}$ (for a proof see for example remark 2.10 ). The symplectic projectors $\Pi_{V_{n, \sigma}}, \sigma \in\{ \pm\}$, on the symplectic subspaces $V_{n, \sigma}$ are explicitly provided by (2.33) and (2.34) with $j=n \sigma$.

Note that the involution $\mathcal{S}$ defined in (2.4) and the translation operator $\tau_{\varsigma}$ in (2.7) leave the subspaces $V_{n, \sigma}, \sigma \in\{ \pm\}$, invariant.

\subsection{Tangential and Normal Subspaces of the Phase Space}

We decompose the phase space $\mathfrak{H}$ of (2.14) into a direct sum of tangential and normal Lagrangian subspaces $\mathfrak{H}_{\mathbb{S}^{+}, \Sigma}^{\top}$ and $\mathfrak{H}_{\mathbb{S}^{+}, \Sigma}^{<}$. Note that the main part of the solutions (1.19) that we shall obtain in Theorem 1.5 is the component in the tangential subspace $\mathfrak{H}_{\mathbb{S}^{+}, \Sigma}^{\top}$, whereas the component in the normal subspace $\mathfrak{H}_{\mathbb{S}^{+}, \Sigma}^{L}$ is much smaller.

Recalling the definition of the sets $\mathbb{S}^{+}$and $\Sigma$ defined in (1.15) respectively (1.16), we split

$$
\mathfrak{H}=\mathfrak{H}_{\mathbb{S}^{+}, \Sigma}^{\top} \oplus \mathfrak{H}_{\mathbb{S}^{+}, \Sigma}^{L}
$$

where $\mathfrak{H}_{\mathbb{S}^{+}, \Sigma}^{\top}$ is the finite dimensional tangential subspace

$$
\mathfrak{H}_{\mathbb{S}^{+}, \Sigma}^{\top}:=\sum_{a=1}^{v} V_{\bar{n}_{a}, \sigma_{a}}
$$

and $\mathfrak{H}_{\mathbb{S}^{+}, \Sigma}^{\angle}$ is the normal subspace defined as its symplectic orthogonal

$$
\mathfrak{H}_{\mathbb{S}^{+}, \Sigma}^{<}:=\sum_{a=1}^{v} V_{\bar{n}_{a},-\sigma_{a}} \oplus \sum_{n \in \mathbb{N}_{\mathbb{S}^{+}}}\left(V_{n,+} \oplus V_{n,-}\right) .
$$

Both the subspaces $\mathfrak{H}_{\mathbb{S}^{+}, \Sigma}^{\top}$ and $\mathfrak{H}_{\mathbb{S}^{+}, \Sigma}^{<}$are Lagrangian. We denote by $\Pi_{\mathbb{S}^{+}, \Sigma}^{\top}$ and $\Pi_{\mathbb{S}^{+}, \Sigma}^{<}$the symplectic projections on the subspaces $\mathfrak{H}_{\mathbb{S}^{+}, \Sigma}^{\top}$ and $\mathfrak{H}_{\mathbb{S}^{+}, \Sigma}^{\angle}$, respectively. Since $\mathfrak{H}_{\mathbb{S}^{+}, \Sigma}^{\top}$ and $\mathfrak{H}_{\mathbb{S}^{+}, \Sigma}^{<}$are symplectic orthogonal, the symplectic form $\mathcal{W}$ in (2.15) decomposes, for any $v_{1}, v_{2} \in \mathfrak{H}_{\mathbb{S}^{+}, \Sigma}^{\top}$ and $w_{1}, w_{2} \in \mathfrak{H}_{\mathbb{S}^{+}, \Sigma}^{L}$, as

$$
\mathcal{W}\left(v_{1}+w_{1}, v_{2}+w_{2}\right)=\mathcal{W}\left(v_{1}, v_{2}\right)+\mathcal{W}\left(w_{1}, w_{2}\right) \text {. }
$$

The symplectic projections $\Pi_{\mathbb{S}^{+}, \Sigma}^{\top}$ and $\Pi_{\mathbb{S}^{+}, \Sigma}^{<}$satisfy

Lemma 2.5. We have that

$$
\begin{array}{ll}
\Pi_{\mathbb{S}^{+}, \Sigma}^{\top} J=J\left(\Pi_{\mathbb{S}^{+}, \Sigma}^{\top}\right)^{*}, \quad\left(\Pi_{\mathbb{S}^{+}, \Sigma}^{\top}\right)^{*} J^{-1}=J^{-1} \Pi_{\mathbb{S}^{+}, \Sigma}^{\top} \\
\Pi_{\mathbb{S}^{+}, \Sigma}^{<} J=J\left(\Pi_{\mathbb{S}^{+}, \Sigma}^{<}\right)^{*}, \quad\left(\Pi_{\mathbb{S}^{+}, \Sigma}^{<}\right)^{*} J^{-1}=J^{-1} \Pi_{\mathbb{S}^{+}, \Sigma}^{<}
\end{array}
$$


Proof. Since the subspaces $\mathfrak{H}^{\top}:=\mathfrak{H}_{\mathbb{S}^{+}, \Sigma}^{\top}$ and $\mathfrak{H}^{\angle}:=\mathfrak{H}_{\mathbb{S}^{+}, \Sigma}^{L}$ are symplectic orthogonal, we have, recalling (2.15), that

$$
\left(J^{-1} v, w\right)_{L^{2}}=\left(J^{-1} w, v\right)_{L^{2}}=0, \quad \forall v \in \mathfrak{H}^{\top}, \forall w \in \mathfrak{H}^{<} .
$$

Thus, using the projectors $\Pi^{\top}:=\Pi_{\mathbb{S}^{+}, \Sigma}^{\top}, \Pi^{<}:=\Pi_{\mathbb{S}^{+}, \Sigma}^{<}$, we have that

$$
\left(J^{-1} \Pi^{\top} v, \Pi^{<} w\right)_{L^{2}}=\left(J^{-1} \Pi^{<} w, \Pi^{\top} v\right)_{L^{2}}=0, \quad \forall v, w \in \mathfrak{H},
$$

and, taking adjoints, $\left(\left(\Pi^{<}\right)^{*} J^{-1} \Pi^{\top} v, w\right)_{L^{2}}=\left(\left(\Pi^{\top}\right)^{*} J^{-1} \Pi^{<} w, v\right)_{L^{2}}=0$ for any $v, w \in \mathfrak{H}$, so that

$$
\left(\Pi^{<}\right)^{*} J^{-1} \Pi^{\top}=0=\left(\Pi^{\top}\right)^{*} J^{-1} \Pi^{<} .
$$

Now inserting the identity $\Pi^{<}=\mathrm{Id}-\Pi^{\top}$ in (2.45), we get

$$
J^{-1} \Pi^{\top}=\left(\Pi^{\top}\right)^{*} J^{-1} \Pi^{\top}=\left(\Pi^{\top}\right)^{*} J^{-1}
$$

proving the second identity of (2.43). The first identity of (2.43) follows applying $J$ to the left and to the right of the second identity. The identity (2.44) follows in the same way.

Note that the restricted symplectic form $\left.\mathcal{W}\right|_{\mathfrak{H}_{\mathbb{S}^{+}, \Sigma}^{<}}$is represented by the symplectic structure

$$
J_{\angle}^{-1}: \mathfrak{H}_{\mathbb{S}^{+}, \Sigma}^{L} \rightarrow \mathfrak{H}_{\mathbb{S}^{+}, \Sigma}^{L}, \quad J_{\angle}^{-1}:=\Pi_{\angle}^{L^{2}} J_{\mid \mathfrak{H}_{\mathbb{S}^{+}, \Sigma}^{<}}^{-1},
$$

where $\Pi_{L}^{L^{2}}$ is the $L^{2}$-projector on the subspace $\mathfrak{H}_{\mathbb{S}^{+}, \Sigma}^{L}$. Indeed

$$
\left.\mathcal{W}\right|_{\mathfrak{H}_{\mathbb{S}^{+}, \Sigma}^{L}}(w, \hat{w})=\left(J_{\angle}^{-1} w, \widehat{w}\right)_{L^{2}}=\left(J^{-1} w, \widehat{w}\right)_{L^{2}}, \quad \forall w, \hat{w} \in \mathfrak{H}_{\mathbb{S}^{+}, \Sigma}^{L} .
$$

We also denote the associated (restricted) Poisson tensor

$$
J_{\angle}: \mathfrak{H}_{\mathbb{S}^{+}, \Sigma}^{L} \rightarrow \mathfrak{H}_{\mathbb{S}^{+}, \Sigma}^{L}, \quad J_{\angle}:=\Pi_{\mathbb{S}^{+}, \Sigma}^{L} J_{\mid \mathfrak{H}_{\mathbb{S}^{+}, \Sigma}^{L}} .
$$

In the next lemma we prove that $J_{\angle}^{-1}$ and $J_{\angle}$ are each other inverses.

Lemma 2.6. $J_{\angle}^{-1} J_{\angle}=J_{\angle} J_{\angle}^{-1}=\operatorname{Id}_{\mathfrak{H}_{\mathbb{S}^{+}, \Sigma}}$.

Proof. Let $v \in \mathfrak{H}_{\mathbb{S}^{+}, \Sigma}^{L}$. By (2.46) and (2.47), for any $h \in \mathfrak{H}_{\mathbb{S}^{+}, \Sigma}^{L}$ one has

$$
\begin{aligned}
\left(J_{\angle}^{-1} J_{L} v, h\right)_{L^{2}} & =\left(J^{-1} \Pi_{\mathbb{S}^{+}, \Sigma}^{<} J v, \Pi_{\angle}^{L^{2}} h\right)_{L^{2}}=-\left(\Pi_{\mathbb{S}^{+}, \Sigma}^{<} J v, J^{-1} h\right)_{L^{2}} \\
& =-\left(J v,\left(\Pi_{\mathbb{S}^{+}, \Sigma}^{<}\right)^{*} J^{-1} h\right)_{L^{2}} \stackrel{(2.44)}{=}-\left(J v, J^{-1} \Pi_{\mathbb{S}^{+}, \Sigma}^{<} h\right)_{L^{2}}=(v, h)_{L^{2}} .
\end{aligned}
$$

The proof that $J_{\angle} J_{\angle}^{-1}=\operatorname{Id}_{\mathfrak{H}_{\mathbb{S}^{+}, \Sigma}}$ is similar.

Lemma 2.7. $\Pi_{\mathbb{S}^{+}, \Sigma}^{\angle} J \Pi_{\angle}^{L^{2}}=\Pi_{\mathbb{S}^{+}, \Sigma}^{\angle} J$.

Proof. For any $u, h \in \mathfrak{H}$ we have, using Lemma 2.5,

$$
\begin{aligned}
\left(\Pi_{\mathbb{S}^{+}, \Sigma}^{<} J \Pi_{\angle}^{L^{2}} u, h\right)_{L^{2}} & =-\left(\Pi_{\angle}^{L^{2}} u, J\left(\Pi_{\mathbb{S}^{+}, \Sigma}^{<}\right)^{*} h\right)_{L^{2}}=-\left(\Pi_{\angle}^{L^{2}} u, \Pi_{\mathbb{S}^{+}, \Sigma}^{<} J h\right)_{L^{2}} \\
& =-\left(u, \Pi_{\mathbb{S}^{+}, \Sigma}^{<} J h\right)_{L^{2}}=\left(J\left(\Pi_{\mathbb{S}^{+}, \Sigma}^{<}\right)^{*} u, h\right)_{L^{2}}=\left(\Pi_{\mathbb{S}^{+}, \Sigma}^{<} J u, h\right)_{L^{2}},
\end{aligned}
$$

implying the lemma. 
Action-angle coordinates. Finally we introduce action-angle coordinates on the tangential subspace $\mathfrak{H}_{\mathbb{S}^{+}, \Sigma}^{\top}$ defined in (2.41). Given the sets $\mathbb{S}^{+}$and $\Sigma$ defined respectively in (1.15) and (1.16), we define the set

$$
\mathbb{S}:=\left\{\bar{J}_{1}, \ldots, \bar{J}_{\nu}\right\} \subset \mathbb{Z} \backslash\{0\}, \quad \bar{J}_{a}:=\sigma_{a} \bar{n}_{a}, \quad a=1, \ldots, v,
$$

and the action-angle coordinates $\left(\theta_{j}, I_{j}\right)_{j \in \mathbb{S}}$, by the relations, for any $j \in \mathbb{S}$,

$$
\alpha_{j}=\sqrt{\frac{1}{\pi}\left(I_{j}+\xi_{j}\right)} \cos \left(\theta_{j}\right), \beta_{j}=-\sqrt{\frac{1}{\pi}\left(I_{j}+\xi_{j}\right)} \sin \left(\theta_{j}\right), \quad \xi_{j}>0,\left|I_{j}\right|<\xi_{j} .
$$

In view of (2.40)-(2.42), we represent any function of the phase space $\mathfrak{H}$ as

$$
\begin{aligned}
& A(\theta, I, w):=v^{\top}(\theta, I)+w, \\
& \quad:=\frac{1}{\sqrt{\pi}} \sum_{j \in \mathbb{S}}\left[\left(\begin{array}{c}
M_{j} \sqrt{I_{j}+\xi_{j}} \cos \left(\theta_{j}\right) \\
-M_{j}^{-1} \sqrt{I_{j}+\xi_{j}} \sin \left(\theta_{j}\right)
\end{array}\right) \cos (j x)+\left(\begin{array}{c}
M_{j} \sqrt{I_{j}+\xi_{j}} \sin \left(\theta_{j}\right) \\
M_{j}^{-1} \sqrt{I_{j}+\xi_{j}} \cos \left(\theta_{j}\right)
\end{array}\right) \sin (j x)\right]+w \\
& \quad=\frac{1}{\sqrt{\pi}} \sum_{j \in \mathbb{S}}\left[\left(\begin{array}{c}
M_{j} \sqrt{I_{j}+\xi_{j}} \cos \left(\theta_{j}-j x\right) \\
-M_{j}^{-1} \sqrt{I_{j}+\xi_{j}} \sin \left(\theta_{j}-j x\right)
\end{array}\right)\right]+w
\end{aligned}
$$

where $\theta:=\left(\theta_{j}\right)_{j \in \mathbb{S}} \in \mathbb{T}^{v}, I:=\left(I_{j}\right)_{j \in \mathbb{S}} \in \mathbb{R}^{v}$ and $w \in \mathfrak{H}_{\mathbb{S}^{+}, \Sigma}^{\angle}$.

Remark 2.8. In these coordinates the solutions (1.17) of the linear system (1.6) simply read as $W v^{\top}(\vec{\Omega}(\kappa) t, 0)$, where $\vec{\Omega}(\kappa):=\left(\Omega_{j}(\kappa)\right)_{j \in \mathbb{S}}$ is given in (1.18).

In view of (2.50), the involution $\mathcal{S}$ in (2.4) reads as

$$
\overrightarrow{\mathcal{S}}:(\theta, I, w) \mapsto(-\theta, I, \mathcal{S} w),
$$

the translation operator $\tau_{\varsigma}$ in (2.7) reads as

$$
\vec{\tau}_{\varsigma}:(\theta, I, w) \mapsto\left(\theta-\vec{\jmath} \varsigma, I, \tau_{\varsigma} w\right), \quad \forall \varsigma \in \mathbb{R},
$$

where

$$
\vec{J}:=(j)_{j \in \mathbb{S}}=\left(\bar{J}_{1}, \ldots, \bar{J}_{v}\right) \in \mathbb{Z}^{v} \backslash\{0\},
$$

and the symplectic 2 -form (2.15) becomes

$$
\mathcal{W}=\left.\sum_{j \in \mathbb{S}}\left(\mathrm{d} \theta_{j} \wedge \mathrm{d} I_{j}\right) \oplus \mathcal{W}\right|_{\mathfrak{H}_{\mathbb{S}^{+}, \Sigma}^{<}}
$$

We also note that $\mathcal{W}$ is exact, namely

$$
\mathcal{W}=d \Lambda, \quad \text { where } \Lambda_{(\theta, I, w)}[\widehat{\theta}, \widehat{I}, \widehat{w}]:=-\sum_{j \in \mathbb{S}} I_{j} \widehat{\theta}_{j}+\frac{1}{2}\left(J_{\angle}^{-1} w, \widehat{w}\right)_{L^{2}}
$$

is the associated Liouville 1-form (the operator $J_{L}^{-1}$ is defined in (2.46)).

Finally, given a Hamiltonian $K: \mathbb{T}^{v} \times \mathbb{R}^{v} \times \mathfrak{H}_{\mathbb{S}^{+}, \Sigma}^{L} \rightarrow \mathbb{R}$, the associated Hamiltonian vector field (with respect to the symplectic form (2.54)) is

$$
X_{K}:=\left(\partial_{I} K,-\partial_{\theta} K, J_{\angle} \nabla_{w} K\right)=\left(\partial_{I} K,-\partial_{\theta} K, \Pi_{\mathbb{S}^{+}, \Sigma}^{<} J \nabla_{w} K\right),
$$


where $\nabla_{w} K$ denotes the $L^{2}$ gradient of $K$ with respect to $w \in \mathfrak{H}_{\mathbb{S}^{+}, \Sigma}$. Indeed, the only nontrivial component of the vector field $X_{K}$ is the last one, which we denote by $\left[X_{K}\right]_{w} \in \mathfrak{H}_{\mathbb{S}^{+}, \Sigma}^{L}$. It fulfills

$$
\left(J_{\angle}^{-1}\left[X_{K}\right]_{w}, \widehat{w}\right)_{L^{2}}=\mathrm{d}_{w} K[\widehat{w}]=\left(\nabla_{w} K, \widehat{w}\right)_{L^{2}}, \quad \forall \widehat{w} \in \mathfrak{H}_{\mathbb{S}^{+}, \Sigma}^{L},
$$

and (2.56) follows by Lemma 2.6. We remark that along the paper we only consider Hamiltonians such that the $L^{2}$-gradient $\nabla_{w} K$ defined by (2.57), as well as the Hamiltonian vector field $\Pi_{\mathbb{S}^{+}, \Sigma}^{\angle} J \nabla_{w} K$, maps spaces of Sobolev functions into Sobolev functions (not just distributions), with possible loss of derivatives.

Tangential and normal subspaces in complex variables. Each 2-dimensional symplectic subspace $V_{n, \sigma}, n \in \mathbb{N}, \sigma= \pm 1$, defined in (2.39)-(2.39) is isomorphic, through the linear map $\mathcal{M C}$ defined in (2.32), to the complex subspace

$$
\mathbf{H}_{j}:=\left\{\left(\frac{z_{j} e^{\mathrm{i} j x}}{z_{j}} e^{-\mathrm{i} j x}\right), z_{j} \in \mathbb{C}\right\} \quad \text { with } \quad j=n \sigma \in \mathbb{Z} .
$$

Denoting by $\Pi_{j}$ the $L^{2}$-projection on $\mathbf{H}_{j}$, we have that $\Pi_{V_{n, \sigma}}=\mathcal{M C} \Pi_{j}(\mathcal{M C})^{-1}$. Thus $\mathcal{M C}$ is an isomorphism between the tangential subspace $\mathfrak{H}_{\mathbb{S}^{+}, \Sigma}^{\top}$ defined in (2.41) and

$$
\mathbf{H}_{\mathbb{S}}:=\left\{\left(\frac{z}{\bar{z}}\right): z(x)=\sum_{j \in \mathbb{S}} z_{j} e^{\mathrm{i} j x}\right\}
$$

and between the normal subspace $\mathfrak{H}_{\mathbb{S}^{+}, \Sigma}^{L}$ defined in (2.42) and

$$
\mathbf{H}_{\mathbb{S}_{0}}^{\perp}:=\left\{\left(\frac{z}{\bar{z}}\right): z(x)=\sum_{j \in \mathbb{S}_{0}^{c}} z_{j} e^{i j x} \in L^{2}\right\}, \quad \mathbb{S}_{0}^{c}:=\mathbb{Z} \backslash(\mathbb{S} \cup\{0\}) .
$$

Denoting by $\Pi_{\mathbb{S}}^{\top}, \Pi_{\mathbb{S}_{0}}^{\perp}$, the $L^{2}$-orthogonal projections on the subspaces $\mathbf{H}_{\mathbb{S}}$ and $\mathbf{H}_{\mathbb{S}_{0}}^{\perp}$, we have that

$$
\Pi_{\mathbb{S}^{+}, \Sigma}^{\top}=\mathcal{M C} \Pi_{\mathbb{S}}^{\top}(\mathcal{M C})^{-1}, \quad \Pi_{\mathbb{S}^{+}, \Sigma}^{<}=\mathcal{M C} \Pi_{\mathbb{S}_{0}}^{\perp}(\mathcal{M C})^{-1}
$$

The following lemma, used in Section 5, is an easy corollary of the previous analysis.

Lemma 2.9. We have that $\left(v^{\top}, \boldsymbol{\Omega}_{W} w\right)_{L^{2}}=0$, for any $v^{\top} \in \mathfrak{H}_{\mathbb{S}^{+}, \Sigma}^{\top}$ and $w \in \mathfrak{H}_{\mathbb{S}^{+}, \Sigma}^{L}$.

Proof. Write $v^{\top}=\mathcal{M C} z^{\top}$ and $\mathcal{M C} z^{\perp}$ with $z^{\top} \in \mathbf{H}_{\mathbb{S}}$ and $z^{\perp} \in \mathbf{H}_{\mathbb{S}_{0}}^{\perp}$. Then, by (2.22) and (2.25),

$$
\left(v^{\top}, \boldsymbol{\Omega}_{W} w\right)_{L^{2}}=\left(\mathcal{M C} z^{\top}, \boldsymbol{\Omega}_{W} \mathcal{M C} z^{\perp}\right)_{L^{2}}=\left(z^{\top}, \boldsymbol{\Omega}_{D} z^{\perp}\right)_{L^{2}}=0,
$$

since $\boldsymbol{\Omega}_{D}$ preserves the subspace $\mathbf{H}_{\mathbb{S}_{0}}^{\perp}$.

Remark 2.10. The same proof of Lemma 2.9 actually shows that $\left(v_{n,-\sigma}\right.$, $\left.\boldsymbol{\Omega}_{W} v_{n, \sigma}\right)_{L^{2}}=0$ for any $v_{n, \pm \sigma} \in V_{n, \pm \sigma}$, for any $n \in \mathbb{N}, \sigma= \pm 1$. Thus $\mathcal{W}\left(v_{n,-\sigma}\right.$ $\left.J \boldsymbol{\Omega}_{W} v_{n, \sigma}\right)=\left(v_{n,-\sigma}, J^{-1} \boldsymbol{J} \boldsymbol{\Omega}_{W} v_{n, \sigma}\right)_{L^{2}}=0$ which shows that $J \boldsymbol{\Omega}_{W}$ maps $V_{n, \sigma}$ in itself.

Notation. The notation $a \lesssim_{s} b$ means that $a \leqq C(s) b$ for some positive constant $C(s)$. We denote $\mathbb{N}:=\{1,2, \ldots\}$ and $\mathbb{N}_{0}:=\{0\} \cup \mathbb{N}$. 


\section{Functional Setting}

Along the paper we consider functions $u(\varphi, x) \in L^{2}\left(\mathbb{T}^{\nu+1}, \mathbb{C}\right)$ depending on the space variable $x \in \mathbb{T}=\mathbb{T}_{x}$ and the angles $\varphi \in \mathbb{T}^{\nu}=\mathbb{T}_{\varphi}^{\nu}$ (so that $\mathbb{T}^{\nu+1}=$ $\mathbb{T}_{\varphi}^{v} \times \mathbb{T}_{x}$ ) which we expand in Fourier series as

$$
u(\varphi, x)=\sum_{j \in \mathbb{Z}} u_{j}(\varphi) e^{\mathrm{i} j x}=\sum_{\ell \in \mathbb{Z}^{v}, j \in \mathbb{Z}} u_{\ell, j} e^{\mathrm{i}(\ell \cdot \varphi+j x)} .
$$

We also consider real valued functions $u(\varphi, x) \in \mathbb{R}$, as well as vector valued functions $u(\varphi, x) \in \mathbb{C}^{2}$ (or $u(\varphi, x) \in \mathbb{R}^{2}$ ). When no confusion appears, we denote simply by $L^{2}, L^{2}\left(\mathbb{T}^{\nu+1}\right), L_{x}^{2}:=L^{2}\left(\mathbb{T}_{x}\right), L_{\varphi}^{2}:=L^{2}\left(\mathbb{T}^{v}\right)$ either the spaces of real/complex valued, scalar/vector valued, $L^{2}$-functions.

In this paper a crucial role is played by the following subspace of functions of $(\varphi, x)$.

Definition 3.1. (Quasi-periodic traveling waves) Let $\vec{j}:=\left(\bar{J}_{1}, \ldots, \bar{J}_{\nu}\right) \in \mathbb{Z}^{v}$ be the vector defined in (2.53). A function $u(\varphi, x)$ is called a quasi-periodic traveling wave if it has the form $u(\varphi, x)=U(\varphi-\vec{\jmath} x)$ where $U: \mathbb{T}^{\nu} \rightarrow \mathbb{C}^{K}, K \in \mathbb{N}$, is a $(2 \pi)^{\nu}$-periodic function.

Comparing with Definition 1.2, we find convenient to call quasi-periodic traveling wave both the function $u(\varphi, x)=U(\varphi-\vec{J} x)$ and the function of time $u(\omega t, x)=U(\omega t-\vec{\jmath} x)$.

Quasi-periodic traveling waves are characterized by the relation

$$
u(\varphi-\vec{\jmath} \varsigma, \cdot)=\tau_{\varsigma} u \quad \forall \varsigma \in \mathbb{R},
$$

where $\tau_{\zeta}$ is the translation operator in (2.7). Product and composition of quasiperiodic traveling waves is a quasi-periodic traveling wave. Expanded in Fourier series as in (3.1), a quasi-periodic traveling wave has the form

$$
u(\varphi, x)=\sum_{\ell \in \mathbb{Z}^{v}, j \in \mathbb{Z}, j+\vec{\jmath} \cdot \ell=0} u_{\ell, j} e^{\mathrm{i}(\ell \cdot \varphi+j x)},
$$

namely, comparing with Definition 3.1,

$$
u(\varphi, x)=U(\varphi-\vec{\jmath} x), \quad U(\psi)=\sum_{\ell \in \mathbb{Z}^{v}} U_{\ell} e^{\mathrm{i} \ell \cdot \psi}, \quad U_{\ell}=u_{\ell,-\vec{\jmath} \cdot \ell} .
$$

The traveling waves $u(\varphi, x)=U(\varphi-\vec{J} x)$ where $U(\cdot)$ belongs to the Sobolev space $H^{s}\left(\mathbb{T}^{v}, \mathbb{C}^{K}\right)$ in (1.14) (with values in $\mathbb{C}^{K}, K \in \mathbb{N}$ ), form a subspace of the Sobolev space

$$
H^{s}\left(\mathbb{T}^{\nu+1}\right)=\left\{u=\sum_{(\ell, j) \in \mathbb{Z}^{\nu+1}} u_{\ell, j} e^{\mathrm{i}(\ell \cdot \varphi+j x)}:\|u\|_{s}^{2}:=\sum_{(\ell, j) \in \mathbb{Z}^{\nu+1}}\left|u_{\ell, j}\right|^{2}\langle\ell, j\rangle^{2 s}<\infty\right\}
$$

where $\langle\ell, j\rangle:=\max \{1,|\ell|,|j|\}$. Note the equivalence of the norms (use (3.4))

$$
\|u\|_{H^{s}\left(\mathbb{T}_{\varphi}^{v} \times \mathbb{T}_{x}\right)} \simeq_{s}\|U\|_{H^{s}\left(\mathbb{T}^{v}\right)} .
$$


For $s \geqq s_{0}:=\left[\frac{\nu+1}{2}\right]+1 \in \mathbb{N}$ one has $H^{s}\left(\mathbb{T}^{\nu+1}\right) \subset C\left(\mathbb{T}^{\nu+1}\right)$, and $H^{s}\left(\mathbb{T}^{\nu+1}\right)$ is an algebra. Along the paper we denote by \|\|$_{s}$ both the Sobolev norms in (1.14) and (3.5).

For $K \geqq 1$ we define the smoothing operator $\Pi_{K}$ on the traveling waves

$$
\Pi_{K}: u=\sum_{\ell \in \mathbb{Z}^{v}, j \in \mathbb{S}_{0}^{c}, j+\vec{\jmath} \cdot \ell=0} u_{\ell, j} e^{\mathrm{i}(\ell \cdot \varphi+j x)} \mapsto \Pi_{K} u=\sum_{\langle\ell\rangle \leqq K, j \in \mathbb{S}_{0}^{c}, j+\vec{\jmath} \cdot \ell=0} u_{\ell, j} e^{\mathrm{i}(\ell \cdot \varphi+j x)},
$$

and $\Pi_{K}^{\perp}:=\mathrm{Id}-\Pi_{K}$. Note that, writing a traveling wave as in (3.4), the projector $\Pi_{K}$ in (3.6) is equal to

$$
\left(\Pi_{K} u\right)(\varphi, x)=U_{K}(\varphi-\vec{\jmath} x), \quad U_{K}(\psi):=\sum_{\ell \in \mathbb{Z}^{\nu},\langle\ell\rangle \leqq K} U_{\ell} e^{i \ell \cdot \psi} .
$$

Whitney-Sobolev functions. Along the paper we consider families of Sobolev functions $\lambda \mapsto u(\lambda) \in H^{s}\left(\mathbb{T}^{\nu+1}\right)$ and $\lambda \mapsto U(\lambda) \in H^{s}\left(\mathbb{T}^{\nu}\right)$ which are $k_{0}$-times differentiable in the sense of Whitney with respect to the parameter $\lambda:=(\omega, \kappa) \in$ $F \subset \mathbb{R}^{v} \times\left[\kappa_{1}, \kappa_{2}\right]$ where $F \subset \mathbb{R}^{v+1}$ is a closed set. The case that we encounter is when $\omega$ belongs to the closed set of Diophantine vectors $\operatorname{DC}(v, \tau)$ defined in (1.13). We refer to Definition 2.1 in [2], for the definition of a Whitney-Sobolev function $u: F \rightarrow H^{s}$ where $H^{s}$ may be either the Hilbert space $H^{s}\left(\mathbb{T}^{v} \times \mathbb{T}\right)$ or $H^{s}\left(\mathbb{T}^{v}\right)$. Here we mention that, given $v \in(0,1)$, we can identify a WhitneySobolev function $u: F \rightarrow H^{s}$ with $k_{0}$ derivatives with the equivalence class of functions $f \in W^{k_{0}, \infty, v}\left(\mathbb{R}^{v+1}, H^{s}\right) / \sim$ with respect to the equivalence relation $f \sim g$ when $\partial_{\lambda}^{j} f(\lambda)=\partial_{\lambda}^{j} g(\lambda)$ for all $\lambda \in F,|j| \leqq k_{0}-1$, with equivalence of the norms

$$
\|u\|_{s, F}^{k_{0}, v} \sim_{\nu, k_{0}}\|u\|_{W^{k_{0}, \infty, v}\left(\mathbb{R}^{v+1}, H^{s}\right)}:=\sum_{|\alpha| \leqq k_{0}} v^{|\alpha|}\left\|\partial_{\lambda}^{\alpha} u\right\|_{L^{\infty}\left(\mathbb{R}^{v+1}, H^{s}\right)} .
$$

The key result is the Whitney extension theorem, which associates to a WhitneySobolev function $u: F \rightarrow H^{s}$ with $k_{0}$-derivatives a function $\widetilde{u}: \mathbb{R}^{v+1} \rightarrow H^{s}$, $\tilde{u}$ in $W^{k_{0}, \infty}\left(\mathbb{R}^{v+1}, H^{s}\right)$ (independently of the target Sobolev space $H^{s}$ ) with an equivalent norm. For sake of simplicity in the notation we often denote \|\|$_{s, F}^{k_{0}, v}=$ \|\|$_{s}^{k_{0}, v}$.

Thanks to this equivalence, all the tame estimates which hold for Sobolev spaces carry over for Whitney-Sobolev functions. For example the following classical tame estimate for the product holds: (see for example Lemma 2.4 in [2]): for all $s \geqq s_{0}>(v+1) / 2$,

$$
\|u v\|_{s}^{k_{0}, v} \leqq C\left(s, k_{0}\right)\|u\|_{s}^{k_{0}, v}\|v\|_{s_{0}}^{k_{0}, v}+C\left(s_{0}, k_{0}\right)\|u\|_{s_{0}}^{k_{0}, v}\|v\|_{s}^{k_{0}, v} .
$$

Moreover the following estimates hold for the smoothing operators defined in (3.6): for any traveling wave $u$

$$
\begin{aligned}
& \left\|\Pi_{K} u\right\|_{s}^{k_{0}, v} \leqq K^{\alpha}\|u\|_{s-\alpha}^{k_{0}, v}, \quad 0 \leqq \alpha \leqq s, \\
& \left\|\Pi_{K}^{\perp} u\right\|_{s}^{k_{0}, v} \leqq K^{-\alpha}\|u\|_{s+\alpha}^{k_{0}, v}, \quad \alpha \geqq 0 .
\end{aligned}
$$


We also state a standard Moser tame estimate for the nonlinear composition operator, see for example Lemma 2.6 in [2],

$$
u(\varphi, x) \mapsto \mathrm{f}(u)(\varphi, x):=f(\varphi, x, u(\varphi, x))
$$

Since the variables $(\varphi, x)=: y$ have the same role, we state it for a generic Sobolev space $H^{s}\left(\mathbb{T}^{d}\right)$.

Lemma 3.2. (Composition operator) Let $f \in \mathcal{C}^{\infty}\left(\mathbb{T}^{d} \times \mathbb{R}, \mathbb{R}\right)$. If $u(\lambda) \in H^{s}\left(\mathbb{T}^{d}\right)$ is a family of Sobolev functions satisfying $\|u\|_{s_{0}}^{k_{0}, v} \leqq 1$, then, for all $s \geqq s_{0}:=$ $(d+1) / 2$,

$$
\|\mathrm{f}(u)\|_{s}^{k_{0}, v} \leqq C\left(s, k_{0}, f\right)\left(1+\|u\|_{s}^{k_{0}, v}\right) .
$$

If $f(\varphi, x, 0)=0$ then $\|\mathrm{f}(u)\|_{s}^{k_{0}, v} \leqq C\left(s, k_{0}, f\right)\|u\|_{s}^{k_{0}, v}$.

Diophantine equation. If $\omega$ is a Diophantine vector in $\operatorname{DC}(v, \tau)$, see (1.13), then the equation $\omega \cdot \partial_{\varphi} v=u$, where $u(\varphi, x)$ has zero average with respect to $\varphi$, has the periodic solution

$$
\left(\omega \cdot \partial_{\varphi}\right)^{-1} u:=\sum_{\ell \in \mathbb{Z}^{\nu} \backslash\{0\}, j \in \mathbb{Z}} \frac{u_{\ell, j}}{\mathrm{i} \omega \cdot \ell} e^{\mathrm{i}(\ell \cdot \varphi+j x)} .
$$

For all $\omega \in \mathbb{R}^{v}$, we define its extension

$$
\left(\omega \cdot \partial_{\varphi}\right)_{\mathrm{ext}}^{-1} u(\varphi, x):=\sum_{(\ell, j) \in \mathbb{Z}^{v+1}} \frac{\chi\left(\omega \cdot \ell v^{-1}\langle\ell\rangle^{\tau}\right)}{\mathrm{i} \omega \cdot \ell} u_{\ell, j} e^{\mathrm{i}(\ell \cdot \varphi+j x)}
$$

where $\chi \in \mathcal{C}^{\infty}(\mathbb{R}, \mathbb{R})$ is an even positive $\mathcal{C}^{\infty}$ cut-off function such that

$$
\chi(\xi)=\left\{\begin{array}{ll}
0 & \text { if }|\xi| \leqq \frac{1}{3} \\
1 & \text { if }|\xi| \geqq \frac{2}{3}
\end{array}, \quad \partial_{\xi} \chi(\xi)>0 \quad \forall \xi \in\left(\frac{1}{3}, \frac{2}{3}\right)\right.
$$

Note that $\left(\omega \cdot \partial_{\varphi}\right)_{\mathrm{ext}}^{-1} u=\left(\omega \cdot \partial_{\varphi}\right)^{-1} u$ for all $\omega \in \operatorname{DC}(v, \tau)$. Moreover, if $u(\varphi, x)$ is a quasi-periodic traveling wave with zero average with respect to $\varphi$, then, by (3.3), we see that $\left(\omega \cdot \partial_{\varphi}\right)_{\mathrm{ext}}^{-1} u(\varphi, x)$ is a quasi-periodic traveling wave. It holds that

$$
\left\|\left(\omega \cdot \partial_{\varphi}\right)_{\mathrm{ext}}^{-1} u\right\|_{s, \mathbb{R}^{v+1}}^{k_{0}, v} \leqq C\left(k_{0}\right) v^{-1}\|u\|_{s+\mu, \mathbb{R}^{v+1}}^{k_{0}, v}, \quad \mu:=k_{0}+\tau\left(k_{0}+1\right),
$$

and, for $F \subseteq \operatorname{DC}(v, \tau) \times \mathbb{R}_{+}$, one has $\left\|\left(\omega \cdot \partial_{\varphi}\right)^{-1} u\right\|_{s, F}^{k_{0}, v} \leqq C\left(k_{0}\right) v^{-1}\|u\|_{s+\mu, F}^{k_{0}, v}$. 
Linear operators. Along the paper we consider $\varphi$-dependent families of linear operators $A: \mathbb{T}^{v} \mapsto \mathcal{L}\left(L^{2}\left(\mathbb{T}_{x}\right)\right), \varphi \mapsto A(\varphi)$, acting on subspaces of $L^{2}\left(\mathbb{T}_{x}\right)$, either real or complex valued. We also regard $A$ as an operator (which for simplicity we denote by $A$ as well) that acts on functions $u(\varphi, x)$ of space and time; that is

$$
(A u)(\varphi, x):=(A(\varphi) u(\varphi, \cdot))(x) .
$$

The action of an operator $A$ as in (3.12) on a scalar function $u(\varphi, x) \in L^{2}$ expanded as in (3.1) is

$$
\begin{aligned}
A u(\varphi, x) & =\sum_{j, j^{\prime} \in \mathbb{Z}} A_{j}^{j^{\prime}}(\varphi) u_{j^{\prime}}(\varphi) e^{\mathrm{i} j x} \\
& =\sum_{j, j^{\prime} \in \mathbb{Z}} \sum_{\ell, \ell^{\prime} \in \mathbb{Z}^{v}} A_{j}^{j^{\prime}}\left(\ell-\ell^{\prime}\right) u_{\ell^{\prime}, j^{\prime}} e^{\mathrm{i}(\ell \cdot \varphi+j x)} .
\end{aligned}
$$

We identify an operator $A$ with its matrix $\left(A_{j}^{j^{\prime}}\left(\ell-\ell^{\prime}\right)\right)_{j, j^{\prime} \in \mathbb{Z}, \ell, \ell^{\prime} \in \mathbb{Z}^{\nu}}$, which is Töplitz with respect to the index $\ell$. In this paper we always consider Töplitz operators as in (3.12), (3.13).

Real operators. A linear operator $A$ is real if $A=\bar{A}$, where $\bar{A}$ is defined by $\bar{A}(u):=\overline{A(\bar{u})}$. Equivalently $A$ is real if it maps real valued functions into real valued functions. We represent a real operator acting on $(\eta, \zeta)$ belonging to (a subspace of) $L^{2}\left(\mathbb{T}_{x}, \mathbb{R}^{2}\right.$ ) by a matrix

$$
\mathcal{R}=\left(\begin{array}{ll}
A & B \\
C & D
\end{array}\right)
$$

where $A, B, C, D$ are real operators acting on the scalar valued components $\eta, \zeta \in$ $L^{2}\left(\mathbb{T}_{x}, \mathbb{R}\right)$.

The change of coordinates (2.24) transforms the real operator $\mathcal{R}$ into a complex one acting on the variables $(z, \bar{z})$, given by the matrix

$$
\begin{aligned}
\mathbf{R} & :=\mathcal{C}^{-1} \mathcal{R C}=\left(\frac{\mathcal{R}_{1}}{\mathcal{R}_{2}} \frac{\mathcal{R}_{2}}{\mathcal{R}_{1}}\right), \\
\mathcal{R}_{1} & :=\frac{1}{2}\{(A+D)-\mathrm{i}(B-C)\}, \quad \mathcal{R}_{2}:=\frac{1}{2}\{(A-D)+\mathrm{i}(B+C)\} .
\end{aligned}
$$

A matrix operator acting on the complex variables $(z, \bar{z})$ of the form (3.15), we call it real. We shall also consider real operators $\mathbf{R}$ of the form (3.15) acting on subspaces of $L^{2}$.

Lie expansion. Let $X(\varphi)$ be a linear operator with associated flow $\Phi^{\tau}(\varphi)$ defined by

$$
\left\{\begin{array}{l}
\partial_{\tau} \Phi^{\tau}(\varphi)=X(\varphi) \Phi^{\tau}(\varphi) \\
\Phi^{0}(\varphi)=\mathrm{Id},
\end{array} \quad \tau \in[0,1] .\right.
$$

Let $\Phi(\varphi):=\Phi^{\tau}(\varphi)_{\mid \tau=1}$ denote the time-1 flow. Given a linear operator $A(\varphi)$, the conjugated operator

$$
A^{+}(\varphi):=\Phi(\varphi)^{-1} A(\varphi) \Phi(\varphi)
$$


admits the Lie expansion; that is for any $M \in \mathbb{N}_{0}$,

$$
\begin{aligned}
& A^{+}(\varphi)=\sum_{m=0}^{M} \frac{(-1)^{m}}{m !} \operatorname{ad}_{X(\varphi)}^{m}(A(\varphi))+R_{M}(\varphi), \\
& R_{M}(\varphi)=\frac{(-1)^{M+1}}{M !} \int_{0}^{1}(1-\tau)^{M}\left(\Phi^{\tau}(\varphi)\right)^{-1} \operatorname{ad}_{X(\varphi)}^{M+1}(A(\varphi)) \Phi^{\tau}(\varphi) \mathrm{d} \tau,
\end{aligned}
$$

where $\operatorname{ad}_{X(\varphi)}(A(\varphi)):=[X(\varphi), A(\varphi)]=X(\varphi) A(\varphi)-A(\varphi) X(\varphi)$ and $\operatorname{ad}_{X(\varphi)}^{0}:=$ Id.

In particular, for $A=\omega \cdot \partial_{\varphi}$, since $\left[X(\varphi), \omega \cdot \partial_{\varphi}\right]=-\left(\omega \cdot \partial_{\varphi} X\right)(\varphi)$, we obtain

$$
\begin{gathered}
\Phi(\varphi)^{-1} \circ \omega \cdot \partial_{\varphi} \circ \Phi(\varphi)=\omega \cdot \partial_{\varphi}+\sum_{m=1}^{M} \frac{(-1)^{m+1}}{m !} \operatorname{ad}_{X(\varphi)}^{m-1}\left(\omega \cdot \partial_{\varphi} X(\varphi)\right) \\
+\frac{(-1)^{M}}{M !} \int_{0}^{1}(1-\tau)^{M}\left(\Phi^{\tau}(\varphi)\right)^{-1} \operatorname{ad}_{X(\varphi)}^{M}\left(\omega \cdot \partial_{\varphi} X(\varphi)\right) \Phi^{\tau}(\varphi) \mathrm{d} \tau
\end{gathered}
$$

For matrices of operators $\mathbf{X}(\varphi)$ and $\mathbf{A}(\varphi)$ as in (3.15), the same formula (3.16) holds.

\subsection{Pseudodifferential Calculus}

In this section we report fundamental notions of pseudodifferential calculus, following [6].

Definition 3.3. ( $\Psi D O)$ A pseudodifferential symbol $a(x, j)$ of order $m$ is the restriction to $\mathbb{R} \times \mathbb{Z}$ of a function $a(x, \xi)$ which is $\mathcal{C}^{\infty}$-smooth on $\mathbb{R} \times \mathbb{R}, 2 \pi$-periodic in $x$, and satisfies

$$
\left|\partial_{x}^{\alpha} \partial_{\xi}^{\beta} a(x, \xi)\right| \leqq C_{\alpha, \beta}\langle\xi\rangle^{m-\beta}, \quad \forall \alpha, \beta \in \mathbb{N}_{0} .
$$

We denote by $S^{m}$ the class of symbols of order $m$ and $S^{-\infty}:=\cap_{m \geqq 0} S^{m}$. To a symbol $a(x, \xi)$ in $S^{m}$ we associate its quantization acting on a $2 \pi$-periodic function $u(x)=\sum_{j \in \mathbb{Z}} u_{j} e^{\mathrm{i} j x}$ as

$$
[\mathrm{Op}(a) u](x):=\sum_{j \in \mathbb{Z}} a(x, j) u_{j} e^{\mathrm{i} j x} .
$$

We denote by OP $S^{m}$ the set of pseudodifferential operators of order $m$ and OP $S^{-\infty}$ $:=\bigcap_{m \in \mathbb{R}}$ OP $S^{m}$. For a matrix of pseudodifferential operators

$$
\mathbf{A}=\left(\begin{array}{ll}
A_{1} & A_{2} \\
A_{3} & A_{4}
\end{array}\right), \quad A_{i} \in \mathrm{OPS}^{m}, \quad i=1, \ldots, 4,
$$

we say that $\mathbf{A} \in \mathrm{OPS}^{m}$. 
When the symbol $a(x)$ is independent of $\xi$, the operator Op $(a)$ is the multiplication operator by the function $a(x)$, that is $\mathrm{Op}(a): u(x) \mapsto a(x) u(x)$. In such a case we also denote $\mathrm{Op}(a)=a(x)$.

We shall use the following notation, used also in $[1,2,6]$. For any $m \in \mathbb{R} \backslash\{0\}$, we set

$$
|D|^{m}:=\operatorname{Op}\left(\chi(\xi)|\xi|^{m}\right),
$$

where $\chi$ is an even, positive $\mathcal{C}^{\infty}$ cut-off satisfying (3.10). We also identify the Hilbert transform $\mathcal{H}$, acting on the $2 \pi$-periodic functions, defined by

$$
\mathcal{H}\left(e^{\mathrm{i} j x}\right):=-\mathrm{i} \operatorname{sign}(j) e^{\mathrm{i} j x} \quad \forall j \neq 0, \quad \mathcal{H}(1):=0,
$$

with the Fourier multiplier $\mathrm{Op}(-\mathrm{i} \operatorname{sign}(\xi) \chi(\xi))$. Similarly we regard the operator

$$
\partial_{x}^{-1}\left[e^{\mathrm{i} j x}\right]:=-\mathrm{i} j^{-1} e^{\mathrm{i} j x} \quad \forall j \neq 0, \quad \partial_{x}^{-1}[1]:=0,
$$

as the Fourier multiplier $\partial_{x}^{-1}=\mathrm{Op}\left(-\mathrm{i} \chi(\xi) \xi^{-1}\right)$ and the projector $\pi_{0}$, defined on the $2 \pi$-periodic functions as

$$
\pi_{0} u:=\frac{1}{2 \pi} \int_{\mathbb{T}} u(x) d x,
$$

with the Fourier multiplier $\operatorname{Op}(1-\chi(\xi))$. Finally we define, for any $m \in \mathbb{R} \backslash\{0\}$,

$$
\langle D\rangle^{m}:=\pi_{0}+|D|^{m}:=\operatorname{Op}\left((1-\chi(\xi))+\chi(\xi)|\xi|^{m}\right) .
$$

Along the paper we consider families of pseudodifferential operators with a symbol $a(\lambda ; \varphi, x, \xi)$ which is $k_{0}$-times differentiable with respect to a parameter $\lambda:=$ $(\omega, \kappa)$ in an open subset $\Lambda_{0} \subset \mathbb{R}^{v} \times\left[\kappa_{1}, \kappa_{2}\right]$. Note that $\partial_{\lambda}^{k} A=\operatorname{Op}\left(\partial_{\lambda}^{k} a\right)$ for any $k \in \mathbb{N}_{0}^{v+1}$.

We recall the pseudodifferential norm introduced in Definition 2.11 in [6].

Definition 3.4. (Weighted $\Psi D O$ norm) Let $A(\lambda):=a(\lambda ; \varphi, x, D) \in$ OP $^{m}$ be a family of pseudodifferential operators with symbol $a(\lambda ; \varphi, x, \xi) \in S^{m}, m \in \mathbb{R}$, which are $k_{0}$-times differentiable with respect to $\lambda \in \Lambda_{0} \subset \mathbb{R}^{v+1}$. For $v \in(0,1)$, $\alpha \in \mathbb{N}_{0}, s \geqq 0$, we define

$$
\|A\|_{m, s, \alpha}^{k_{0}, v}:=\sum_{|k| \leqq k_{0}} v^{|k|} \sup _{\lambda \in \Lambda_{0}}\left\|\partial_{\lambda}^{k} A(\lambda)\right\|_{m, s, \alpha},
$$

where $\|A(\lambda)\|_{m, s, \alpha}:=\max _{0 \leqq \beta \leqq \alpha} \sup _{\xi \in \mathbb{R}}\left\|\partial_{\xi}^{\beta} a(\lambda, \cdot, \cdot, \xi)\right\|_{s}\langle\xi\rangle^{-m+\beta}$. For a matrix of pseudodifferential operators $\mathbf{A} \in \mathrm{OPS}^{m}$ as in (3.18), we define $\|\mathbf{A}\|_{m, s, \alpha}^{k_{0}, v}:=$ $\max _{i=1, \ldots, 4}\left\|A_{i}\right\|_{m, s, \alpha}^{k_{0}, v}$.

Given a function $a(\lambda ; \varphi, x) \in \mathcal{C}^{\infty}$ which is $k_{0}$-times differentiable with respect to $\lambda$, the weighted norm of the corresponding multiplication operator is

$$
\|\mathrm{Op}(a)\|_{0, s, \alpha}^{k_{0}, v}=\|a\|_{s}^{k_{0}, v}, \quad \forall \alpha \in \mathbb{N}_{0} .
$$


Composition of pseudodifferential operators. If $\mathrm{Op}(a), \mathrm{Op}(b)$ are pseudodifferential operators with symbols $a \in S^{m}, b \in S^{m^{\prime}}, m, m^{\prime} \in \mathbb{R}$, then the composition operator $\mathrm{Op}(a) \mathrm{Op}(b)$ is a pseudodifferential operator $\mathrm{Op}(a \# b)$ with symbol $a \# b \in S^{m+m^{\prime}}$. It admits the asymptotic expansion: for any $N \geqq 1$

$$
\begin{aligned}
(a \# b)(\lambda ; \varphi, x, \xi)= & \sum_{\beta=0}^{N-1} \frac{1}{\mathrm{i}^{\beta} \beta !} \partial_{\xi}^{\beta} a(\lambda ; \varphi, x, \xi) \partial_{x}^{\beta} b(\lambda ; \varphi, x, \xi) \\
& +\left(r_{N}(a, b)\right)(\lambda ; \varphi, x, \xi)
\end{aligned}
$$

where $r_{N}(a, b) \in S^{m+m^{\prime}-N}$. The following result is proved in Lemma 2.13 in [6]:

Lemma 3.5. (Composition) Let $A=a(\lambda ; \varphi, x, D), B=b(\lambda ; \varphi, x, D)$ be pseudodifferential operators with symbols $a(\lambda ; \varphi, x, \xi) \in S^{m}, b(\lambda ; \varphi, x, \xi) \in S^{m^{\prime}}$, $m, m^{\prime} \in \mathbb{R}$. Then $A \circ B \in \mathrm{OPS}^{m+m^{\prime}}$ satisfies, for any $\alpha \in \mathbb{N}_{0}, s \geqq s_{0}$,

$$
\begin{array}{r}
\|A B\|_{m+m^{\prime}, s, \alpha}^{k_{0}, v} \lesssim_{m, \alpha, k_{0}} C(s)\|A\|_{m, s, \alpha}^{k_{0}, v}\|B\|_{m^{\prime}, s_{0}+|m|+\alpha, \alpha}^{k_{0}, v} \\
+C\left(s_{0}\right)\|A\|_{m, s_{0}, \alpha}^{k_{0}, v}\|B\|_{m^{\prime}, s+|m|+\alpha, \alpha}^{k_{0}, v}
\end{array}
$$

Moreover, for any integer $N \geqq 1$, the remainder $R_{N}:=\mathrm{Op}\left(r_{N}\right)$ in (3.23) satisfies

$$
\begin{aligned}
\left\|\mathrm{Op}\left(r_{N}(a, b)\right)\right\|_{m+m^{\prime}-N, s, \alpha}^{k_{0}, v} \lesssim & m_{, N, \alpha, k_{0}} C(s)\|A\|_{m, s, N+\alpha}^{k_{0}, v}\|B\|_{m^{\prime}, s_{0}+|m|+2 N+\alpha, N+\alpha}^{k_{0}, v} \\
& +C\left(s_{0}\right)\|A\|_{m, s_{0}, N+\alpha}^{k_{0}, v}\|B\|_{m^{\prime}, s+|m|+2 N+\alpha, N+\alpha}^{k_{0}, v} .
\end{aligned}
$$

Both (3.24)-(3.25) hold with the constant $C\left(s_{0}\right)$ interchanged with $C(s)$.

Analogous estimates hold if $\mathbf{A}$ and $\mathbf{B}$ are matrix operators of the form (3.18).

The commutator between two pseudodifferential operators $\operatorname{Op}(a) \in \mathrm{OP}^{m}$ and $\mathrm{Op}(b) \in \mathrm{OP} S^{m^{\prime}}$ is a pseudodifferential operator in OP $S^{m+m^{\prime}-1}$ with symbol $a \star b \in S^{m+m^{\prime}-1}$, namely $[\mathrm{Op}(a), \mathrm{Op}(b)]=\mathrm{Op}(a \star b)$, that admits, by (3.23), the expansion

$$
\begin{aligned}
& a \star b=-\mathrm{i}\{a, b\}+\widetilde{r_{2}}(a, b), \quad \widetilde{r_{2}}(a, b):=r_{2}(a, b)-r_{2}(b, a) \in S^{m+m^{\prime}-2}, \\
& \text { where }\{a, b\}:=\partial_{\xi} a \partial_{x} b-\partial_{x} a \partial_{\xi} b,
\end{aligned}
$$

is the Poisson bracket between $a(x, \xi)$ and $b(x, \xi)$. As a corollary of Lemma 3.5 we have

Lemma 3.6. (Commutator) Let $A=\mathrm{Op}(a)$ and $B=\mathrm{Op}(b)$ be pseudodifferential operators with symbols $a(\lambda ; \varphi, x, \xi) \in S^{m}, b(\lambda ; \varphi, x, \xi) \in S^{m^{\prime}}, m, m^{\prime} \in \mathbb{R}$. Then the commutator $[A, B]:=A B-B A \in \mathrm{OPS}^{m+m^{\prime}-1}$ satisfies

$$
\begin{aligned}
\|[A, B]\|_{m+m^{\prime}-1, s, \alpha}^{k_{0}, v} \lesssim & { }_{m, m^{\prime}, \alpha, k_{0}} C(s)\|A\|_{m, s+\left|m^{\prime}\right|+\alpha+2, \alpha+1}^{k_{0}, v}\|B\|_{m^{\prime}, s_{0}+|m|+\alpha+2, \alpha+1}^{k_{0}, v} \\
& +C\left(s_{0}\right)\|A\|_{m, s_{0}+\left|m^{\prime}\right|+\alpha+2, \alpha+1}^{k_{0}, v}\|B\|_{m^{\prime}, s+|m|+\alpha+2, \alpha+1}^{k_{0}, v} .
\end{aligned}
$$


Finally we consider the exponential of a pseudodifferential operator of order 0. The following lemma follows as in Lemma 2.12 of [5] (or Lemma 2.17 in [6]).

Lemma 3.7. (Exponential map) If $A:=\operatorname{Op}(a(\lambda ; \varphi, x, \xi))$ is in $\mathrm{OP} S^{0}$, then $e^{A}$ is in $\mathrm{OP}^{0}$ and for any $s \geqq s_{0}, \alpha \in \mathbb{N}_{0}$, there is a constant $C(s, \alpha)>0$ so that

$$
\left\|e^{A}-\operatorname{Id}\right\|_{0, s, \alpha}^{k_{0}, v} \leqq\|A\|_{0, s+\alpha, \alpha}^{k_{0}, v} \exp \left(C(s, \alpha)\|A\|_{0, s_{0}+\alpha, \alpha}^{k_{0}, v}\right) .
$$

The same holds for a matrix $\mathbf{A}$ of the form (3.18) in OP $S^{0}$.

Egorov Theorem. Consider the family of $\varphi$-dependent diffeomorphisms of $\mathbb{T}_{x}$ defined by

$$
y=x+\beta(\varphi, x) \quad \Longleftrightarrow \quad x=y+\breve{\beta}(\varphi, y),
$$

where $\beta(\varphi, x)$ is a small smooth function, and the induced operators

$$
(\mathcal{B} u)(\varphi, x):=u(\varphi, x+\beta(\varphi, x)), \quad\left(\mathcal{B}^{-1} u\right)(\varphi, y):=u(\varphi, y+\breve{\beta}(\varphi, y)) .
$$

Lemma 3.8. (Composition) Let $\|\beta\|_{2 s_{0}+k_{0}+2}^{k_{0}, v} \leqq \delta\left(s_{0}, k_{0}\right)$ small enough. Then the composition operator $\mathcal{B}$ satisfies the tame estimates, for any $s \geqq s_{0}$,

$$
\|\mathcal{B} u\|_{s}^{k_{0}, v} \lesssim_{s, k_{0}}\|u\|_{s+k_{0}}^{k_{0}, v}+\|\beta\|_{s}^{k_{0}, v}\|u\|_{s_{0}+k_{0}+1}^{k_{0}, v}
$$

and the function $\breve{\beta}$ defined in (3.28) by the inverse diffeomorphism satisfies $\|\breve{\beta}\|_{s}^{k_{0}, v} \lesssim s, k_{0}\|\beta\|_{s+k_{0}}^{k_{0}, v}$.

The following result is a small variation of Proposition 2.28 of [5]:

Proposition 3.9. (Egorov) Let $N \in \mathbb{N}$, $q_{0} \in \mathbb{N}_{0}, S>s_{0}$ and assume that $\partial_{\lambda}^{k} \beta(\lambda ; \cdot, \cdot)$ are $\mathcal{C}^{\infty}$ for all $|k| \leqq k_{0}$. There exist constants $\sigma_{N}, \sigma_{N}\left(q_{0}\right)>0, \delta=\delta\left(S, N, q_{0}, k_{0}\right) \in$ $(0,1)$ such that, if $\|\beta\|_{s_{0}+\sigma_{N}\left(\mathrm{q}_{0}\right)}^{k_{0}, v} \leqq \delta$, then the conjugated operator $\mathcal{B}^{-1} \circ \partial_{x}^{m} \circ \mathcal{B}$, $m \in \mathbb{Z}$, is a pseudodifferential operator of order $m$ with an expansion of the form

$$
\mathcal{B}^{-1} \circ \partial_{x}^{m} \circ \mathcal{B}=\sum_{i=0}^{N} p_{m-i}(\lambda ; \varphi, y) \partial_{y}^{m-i}+\mathcal{R}_{N}(\varphi),
$$

with the following properties:

1. The principal symbol of $p_{m}$ is

$$
p_{m}(\lambda ; \varphi, y)=\left.\left(\left[1+\beta_{x}(\lambda ; \varphi, x)\right]^{m}\right)\right|_{x=y+\breve{\beta}(\lambda ; \varphi, y)}
$$

where $\breve{\beta}(\lambda ; \varphi, y)$ has been introduced in (3.28). For any $s \geqq s_{0}$ and $i=$ $1, \ldots, N$,

$$
\left\|p_{m}-1\right\|_{s}^{k_{0}, v},\left\|p_{m-i}\right\|_{s}^{k_{0}, v} \lesssim_{s, N}\|\beta\|_{s+\sigma_{N}}^{k_{0}, v} .
$$

2. For any $\mathrm{q} \in \mathbb{N}_{0}^{v}$ with $|\mathrm{q}| \leqq \mathrm{q}_{0}, n_{1}, n_{2} \in \mathbb{N}_{0}$ with $n_{1}+n_{2}+\mathrm{q}_{0} \leqq N+1-k_{0}-m$, the operator $\langle D\rangle^{n_{1}} \partial_{\varphi}^{q} \mathcal{R}_{N}(\varphi)\langle D\rangle^{n_{2}}$ is $\mathcal{D}^{k_{0}}$-tame with a tame constant satisfying, for any $s_{0} \leqq s \leqq S$,

$$
\mathfrak{M}_{\langle D\rangle^{n_{1}} \partial_{\varphi}^{\mathrm{q}} \mathcal{R}_{N}(\varphi)\langle D\rangle^{n_{2}}}(s) \lesssim S, N, \mathrm{q}_{0}\|\beta\|_{S+\sigma_{N}\left(\mathrm{q}_{0}\right)}^{k_{0}, v} .
$$


3. Let $s_{0}<s_{1}$ and assume that $\left\|\beta_{j}\right\|_{s_{1}+\sigma_{N}\left(\mathrm{q}_{0}\right)} \leqq \delta, j=1$, 2. Then $\left\|\Delta_{12} p_{m-i}\right\|_{s_{1}}$ $\lesssim_{s_{1}, N}\left\|\Delta_{12} \beta\right\|_{s_{1}+\sigma_{N}}, i=0, \ldots, N$, and, for any $|\mathrm{q}| \leqq \mathrm{q}_{0}, n_{1}, n_{2} \in \mathbb{N}_{0}$ with $n_{1}+n_{2}+\mathrm{q}_{0} \leqq N-m$,

$$
\left\|\langle D\rangle^{n_{1}} \partial_{\varphi}^{\mathrm{q}} \Delta_{12} \mathcal{R}_{N}(\varphi)\langle D\rangle^{n_{2}}\right\|_{\mathcal{B}\left(H^{\left.s_{1}\right)}\right.} \lesssim_{s_{1}, N, n_{1}, n_{2}}\left\|\Delta_{12} \beta\right\|_{s_{1}+\sigma_{N}\left(\mathrm{q}_{0}\right)} .
$$

Finally, if $\beta(\varphi, x)$ is a quasi-periodic traveling wave, then $\mathcal{B}$ is momentum preserving (we refer to Definition 3.24 and Lemma 3.30), as well as the conjugated operator $\mathcal{B}^{-1} \circ \partial_{x}^{m} \circ \mathcal{B}$, and each function $p_{m-i}, i=0, \ldots, N$, is a quasi-periodic traveling wave.

Dirichlet-Neumann operator. We finally remind the following decomposition of the Dirichlet-Neumann operator proved in [6], in the case of infinite depth, and in [2], for finite depth.

Lemma 3.10. (Dirichlet-Neumann) Assume that $\partial_{\lambda}^{k} \eta(\lambda, \cdot, \cdot)$ is $\mathcal{C}^{\infty}\left(\mathbb{T}^{\nu} \times \mathbb{T}_{x}\right)$ for all $|k| \leqq k_{0}$. There exists $\delta\left(s_{0}, k_{0}\right)>0$ such that, if $\|\eta\|_{2 s_{0}+2 k_{0}+1}^{k_{0}, v} \leqq \delta\left(s_{0}, k_{0}\right)$, then the Dirichlet-Neumann operator $G(\eta)=G(\eta, \mathrm{h})$ may be written as

$$
G(\eta, \mathrm{h})=G(0, \mathrm{~h})+\mathcal{R}_{G}(\eta)
$$

where $\mathcal{R}_{G}(\eta):=\mathcal{R}_{G}(\eta, \mathrm{h}) \in \mathrm{OPS}^{-\infty}$ satisfies, for all $m, s, \alpha \in \mathbb{N}_{0}$, the estimate

$$
\left\|\mathcal{R}_{G}(\eta)\right\|_{-m, s, \alpha}^{k_{0}, v} \leqq C\left(s, m, \alpha, k_{0}\right)\|\eta\|_{s+s_{0}+2 k_{0}+m+\alpha+3}^{k_{0}, v} .
$$

\section{2. $\mathcal{D}^{k_{0}}$-Tame and Modulo-Tame Operators}

We present the notion of tame and modulo tame operators introduced in [6]. Let $A:=A(\lambda)$ be a linear operator as in (3.12), $k_{0}$-times differentiable with respect to the parameter $\lambda$ in the open set $\Lambda_{0} \subset \mathbb{R}^{v+1}$.

Definition 3.11. ( $\mathcal{D}^{k_{0}}-\sigma$-tame) Let $\sigma \geqq 0$. A linear operator $A:=A(\lambda)$ is $\mathcal{D}^{k_{0}}-\sigma$ tame if there exists a non-decreasing function $\left[s_{0}, S\right] \rightarrow[0,+\infty), s \mapsto \mathfrak{M}_{A}(s)$, with possibly $S=+\infty$, such that, for all $s_{0} \leqq s \leqq S$ and $u \in H^{s+\sigma}$,

$$
\sup _{|k| \leqq k_{0}} \sup _{\lambda \in \Lambda_{0}} v^{|k|}\left\|\left(\partial_{\lambda}^{k} A(\lambda)\right) u\right\|_{s} \leqq \mathfrak{M}_{A}\left(s_{0}\right)\|u\|_{s+\sigma}+\mathfrak{M}_{A}(s)\|u\|_{s_{0}+\sigma}
$$

We say that $\mathfrak{M}_{A}(s)$ is a tame constant of the operator $A$. The constant $\mathfrak{M}_{A}(s)=$ $\mathfrak{M}_{A}\left(k_{0}, \sigma, s\right)$ may also depend on $k_{0}, \sigma$ but we shall often omit to write them. When the "loss of derivatives" $\sigma$ is zero, we simply write $\mathcal{D}^{k_{0}}$-tame instead of $\mathcal{D}^{k_{0}}$ 0 -tame. For a matrix operator as in (3.15), we denote the tame constant $\mathfrak{M}_{\mathbf{R}}(s):=$ $\max \left\{\mathfrak{M}_{\mathcal{R}_{1}}(s), \mathfrak{M}_{\mathcal{R}_{2}}(s)\right\}$.

Note that the tame constants $\mathfrak{M}_{A}(s)$ are not uniquely determined. An immediate consequence of (3.34) is that $\|A\|_{\mathcal{L}\left(H^{s_{0}+\sigma}, H^{s_{0}}\right)} \leqq 2 \mathfrak{M}_{A}\left(s_{0}\right)$. Also note that, 
representing the operator $A$ by its matrix elements $\left(A_{j}^{j^{\prime}}\left(\ell-\ell^{\prime}\right)\right)_{\ell, \ell^{\prime} \in \mathbb{Z}^{v}, j, j^{\prime} \in \mathbb{Z}}$ as in (3.13), we have for all $|k| \leqq k_{0}, j^{\prime} \in \mathbb{Z}, \ell^{\prime} \in \mathbb{Z}^{v}$,

$$
v^{2|k|} \sum_{\ell, j}\langle\ell, j\rangle^{2 s}\left|\partial_{\lambda}^{k} A_{j}^{j^{\prime}}\left(\ell-\ell^{\prime}\right)\right|^{2} \leqq 2\left(\mathfrak{M}_{A}\left(s_{0}\right)\right)^{2}\left\langle\ell^{\prime}, j^{\prime}\right\rangle^{2(s+\sigma)}+2\left(\mathfrak{M}_{A}(s)\right)^{2}\left\langle\ell^{\prime}, j^{\prime}\right\rangle^{2\left(s_{0}+\sigma\right)} \text {. }
$$

The class of $\mathcal{D}^{k_{0}}-\sigma$-tame operators is closed under composition.

Lemma 3.12. (Composition, Lemma 2.20 in [6]) Let $A, B$ be respectively $\mathcal{D}^{k_{0}}$ $\sigma_{A}$-tame and $\mathcal{D}^{k_{0}}-\sigma_{B}$-tame operators with tame constants respectively $\mathfrak{M}_{A}(s)$ and $\mathfrak{M}_{B}(s)$. Then the composed operator $A \circ B$ is $\mathcal{D}^{k_{0}}-\left(\sigma_{A}+\sigma_{B}\right)$-tame with tame constant

$$
\mathfrak{M}_{A B}(s) \leqq C\left(k_{0}\right)\left(\mathfrak{M}_{A}(s) \mathfrak{M}_{B}\left(s_{0}+\sigma_{A}\right)+\mathfrak{M}_{A}\left(s_{0}\right) \mathfrak{M}_{B}\left(s+\sigma_{A}\right)\right)
$$

It is proved in Lemma 2.22 in [6] that the action of a $\mathcal{D}^{k_{0}}-\sigma$-tame operator $A(\lambda)$ on a Sobolev function $u=u(\lambda) \in H^{s+\sigma}$ is bounded by

$$
\|A u\|_{S}^{k_{0}, v} \lesssim k_{0} \mathfrak{M}_{A}\left(s_{0}\right)\|u\|_{s+\sigma}^{k_{0}, v}+\mathfrak{M}_{A}(s)\|u\|_{s_{0}+\sigma}^{k_{0}, v} .
$$

Pseudodifferential operators are tame operators. We use, in particular, the following lemma:

Lemma 3.13. (Lemma 2.21 in [6]) Let $A=a(\lambda ; \varphi, x, D) \in \mathrm{OP}^{0}$ be a family of pseudodifferential operators satisfying $\|A\|_{0, s, 0}^{k_{0}, v}<\infty$ for $s \geqq s_{0}$. Then $A$ is $\mathcal{D}^{k_{0}}$-tame with a tame constant $\mathfrak{M}_{A}(s)$ satisfying, for any $s \geqq s_{0}$,

$$
\mathfrak{M}_{A}(s) \leqq C(s)\|A\|_{0, s, 0}^{k_{0}, v} .
$$

The same statement holds for a matrix operator $\mathbf{R}$ as in (3.15).

In view of the KAM reducibility scheme of Section 8 we also consider the stronger notion of $\mathcal{D}^{k_{0}}$-modulo-tame operator, that we need only for operators with loss of derivative $\sigma=0$. We first recall the notion of majorant operator: given a linear operator $A$ acting as in (3.13), we define the majorant operator $|A|$ by its

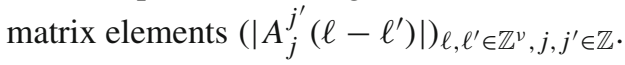

Definition 3.14. ( $\mathcal{D}^{k_{0}}$-modulo-tame) A linear operator $A=A(\lambda)$ is $\mathcal{D}^{k_{0}}$-modulotame if there exists a non-decreasing function $\left[s_{0}, S\right] \rightarrow[0,+\infty], s \mapsto \mathfrak{M}_{A}^{\sharp}(s)$, such that for all $k \in \mathbb{N}_{0}^{\nu+1},|k| \leqq k_{0}$, the majorant operator $\left|\partial_{\lambda}^{k} A\right|$ satisfies, for all $s_{0} \leqq s \leqq S$ and $u \in H^{s}$,

$$
\sup _{|k| \leqq k_{0}} \sup _{\lambda \in \Lambda_{0}} v^{|k|}\left\|\left|\partial_{\lambda}^{k} A\right| u\right\|_{s} \leqq \mathfrak{M}_{A}^{\sharp}\left(s_{0}\right)\|u\|_{s}+\mathfrak{M}_{A}^{\sharp}(s)\|u\|_{s_{0}} .
$$

The constant $\mathfrak{M}_{A}^{\sharp}(s)$ is called a modulo-tame constant for the operator $A$. For a matrix of operators as in (3.15), we denote the modulo-tame constant $\mathfrak{M}_{\mathbf{R}}^{\sharp}(s):=$ $\max \left\{\mathfrak{M}_{\mathcal{R}_{1}}^{\sharp}(s), \mathfrak{M}_{\mathcal{R}_{2}}^{\sharp}(s)\right\}$. 
If $A, B$ are $\mathcal{D}^{k_{0}}$-modulo-tame operators with $\left|A_{j}^{j^{\prime}}(\ell)\right| \leqq\left|B_{j}^{j^{\prime}}(\ell)\right|$, then $\mathfrak{M}_{A}^{\sharp}(s) \leqq$ $\mathfrak{M}_{B}^{\sharp}(s)$. A $\mathcal{D}^{k_{0}}$-modulo-tame operator is also $\mathcal{D}^{k_{0}}$-tame and $\mathfrak{M}_{A}(s) \leqq \mathfrak{M}_{A}^{\sharp}(s)$.

In view of the next lemma, given a linear operator $A$ acting as in (3.13), we define the operator $\left\langle\partial_{\varphi}\right\rangle^{\mathrm{b}} A, \mathrm{~b} \in \mathbb{R}$, whose matrix elements are $\left\langle\ell-\ell^{\prime}\right\rangle^{\mathrm{b}} A_{j}^{j^{\prime}}\left(\ell-\ell^{\prime}\right)$.

Lemma 3.15. (Sum and composition, Lemma 2.25 in [6]) Let $A, B,\left\langle\partial_{\varphi}\right\rangle^{\mathrm{b}} A,\left\langle\partial_{\varphi}\right\rangle^{\mathrm{b}} B$ be $\mathcal{D}^{k_{0}}$-modulo-tame operators. Then $A+B, A \circ B$ and $\left\langle\partial_{\varphi}\right\rangle^{\mathrm{b}}(A B)$ are $\mathcal{D}^{k_{0}}$-modulotame with

$$
\begin{aligned}
& \mathfrak{M}_{A+B}^{\#}(s) \leqq \mathfrak{M}_{A}^{\sharp}(s)+\mathfrak{M}_{B}^{\sharp}(s) \\
& \mathfrak{M}_{A B}^{\sharp}(s) \leqq C\left(k_{0}\right)\left(\mathfrak{M}_{A}^{\sharp}(s) \mathfrak{M}_{B}^{\sharp}\left(s_{0}\right)+\mathfrak{M}_{A}^{\sharp}\left(s_{0}\right) \mathfrak{M}_{B}^{\sharp}(s)\right) \\
& \mathfrak{M}_{\left\langle\partial_{\varphi}\right\rangle^{\mathrm{b}}(A B)}^{\sharp}(s) \leqq C(\mathrm{~b}) C\left(k_{0}\right)\left(\mathfrak{M}_{\left\langle\partial_{\varphi}\right\rangle^{\mathrm{b}} A}^{\sharp}(s) \mathfrak{M}_{B}^{\sharp}\left(s_{0}\right)+\mathfrak{M}_{\left\langle\partial_{\varphi}\right\rangle^{\mathrm{b}} A}^{\sharp}\left(s_{0}\right) \mathfrak{M}_{B}^{\sharp}(s)\right. \\
& \left.\quad+\mathfrak{M}_{A}^{\sharp}(s) \mathfrak{M}_{\left\langle\partial_{\varphi}\right\rangle^{\mathrm{b}} B}^{\sharp}\left(s_{0}\right)+\mathfrak{M}_{A}^{\sharp}\left(s_{0}\right) \mathfrak{M}_{\left\langle\partial_{\varphi}\right\rangle^{\mathrm{b}} B}^{\sharp}(s)\right) .
\end{aligned}
$$

The same statement holds for matrix operators $\mathbf{A}, \mathbf{B}$ as in (3.15).

By Lemma 3.15 we deduce the following result, cfr. Lemma 2.20 in [5].

Lemma 3.16. (Exponential) Let $A$ and $\left\langle\partial_{\varphi}\right\rangle^{\mathrm{b}} A$ be $\mathcal{D}^{k_{0}}$-modulo-tame and assume that $\mathfrak{M}_{A}^{\sharp}\left(s_{0}\right) \leqq 1$. Then the operators $e^{ \pm A}-\operatorname{Id}$ and $\left\langle\partial_{\varphi}\right\rangle^{\mathrm{b}} e^{ \pm A}-\mathrm{Id}$ are $\mathcal{D}^{k_{0}}$-modulotame with modulo-tame constants satisfying

$\mathfrak{M}_{e^{ \pm A}-\mathrm{Id}}^{\sharp}(s) \lesssim k_{0} \mathfrak{M}_{A}^{\sharp}(s), \quad \mathfrak{M}_{\left\langle\partial_{\varphi}\right\rangle^{\mathrm{b}} e^{ \pm A}-\mathrm{Id}}^{\sharp}(s) \lesssim_{k_{0}, \mathrm{~b}} \mathfrak{M}_{\left\langle\partial_{\varphi}\right\rangle^{\mathrm{b}} A}^{\sharp}(s)+\mathfrak{M}_{A}^{\sharp}(s) \mathfrak{M}_{\left\langle\partial_{\varphi}\right\rangle^{\mathrm{b}} A}^{\sharp}\left(s_{0}\right)$.

Given a linear operator $A$ acting as in (3.13), we define the smoothed operator $\Pi_{N} A, N \in \mathbb{N}$ whose matrix elements are

$$
\left(\Pi_{N} A\right)_{j}^{j^{\prime}}\left(\ell-\ell^{\prime}\right):= \begin{cases}A_{j}^{j^{\prime}}\left(\ell-\ell^{\prime}\right) & \text { if }\left\langle\ell-\ell^{\prime}\right\rangle \leqq N \\ 0 & \text { otherwise. }\end{cases}
$$

We also denote $\Pi_{N}^{\perp}:=\mathrm{Id}-\Pi_{N}$. It is proved in Lemma 2.27 in [6] that

$$
\mathfrak{M}_{\Pi_{N}^{\perp} A}^{\sharp}(s) \leqq N^{-\mathrm{b}} \mathfrak{M}_{\left\langle\partial_{\varphi}\right\rangle^{\mathrm{b}} A}^{\sharp}(s), \quad \mathfrak{M}_{\Pi_{N}^{\perp} A}^{\sharp}(s) \leqq \mathfrak{M}_{A}^{\sharp}(s) .
$$

The same estimate holds with a matrix operator $\mathbf{R}$ as in (3.15).

\subsection{Hamiltonian and Reversible Operators}

In this paper we shall exploit both the Hamiltonian and reversible structure along the reduction of the linearized operators, that we now present.

Hamiltonian operators. A matrix operator $\mathcal{R}$ as in (3.14) is Hamiltonian if the matrix

$$
J^{-1} \mathcal{R}=\left(\begin{array}{cc}
0 & -\mathrm{Id} \\
\mathrm{Id} & 0
\end{array}\right)\left(\begin{array}{ll}
A & B \\
C & D
\end{array}\right)=\left(\begin{array}{cc}
-C & -D \\
A & B
\end{array}\right)
$$


is self-adjoint, namely $B^{*}=B, C^{*}=C, A^{*}=-D$ and $A, B, C, D$ are real.

Correspondingly, a matrix operator as in (3.15) is Hamiltonian if

$$
\mathcal{R}_{1}^{*}=-\mathcal{R}_{1}, \quad \mathcal{R}_{2}^{*}=\overline{\mathcal{R}_{2}} .
$$

Symplectic operators. A $\varphi$-dependent family of linear operators $\mathcal{R}(\varphi), \varphi \in \mathbb{T}^{v}$, as in (3.14) is symplectic if

$$
\mathcal{W}(\mathcal{R}(\varphi) u, \mathcal{R}(\varphi) v)=\mathcal{W}(u, v) \quad \forall u, v \in L^{2}\left(\mathbb{T}_{x}, \mathbb{R}^{2}\right),
$$

where the symplectic 2 -form $\mathcal{W}$ is defined in (2.15).

Reversible and reversibility preserving operators. Let $\mathcal{S}$ be an involution as in (2.4) acting on the real variables $(\eta, \zeta) \in \mathbb{R}^{2}$, or as in (2.51) acting on the action-anglenormal variables $(\theta, I, w)$, or as in (2.29) acting in the $(z, \bar{z})$ complex variables introduced in (2.24).

Definition 3.17. (Reversibility) A $\varphi$-dependent family of operators $\mathcal{R}(\varphi), \varphi \in \mathbb{T}^{\nu}$, is

- reversible if $\mathcal{R}(-\varphi) \circ \mathcal{S}=-\mathcal{S} \circ \mathcal{R}(\varphi)$ for all $\varphi \in \mathbb{T}^{\nu}$

- reversibility preserving if $\mathcal{R}(-\varphi) \circ \mathcal{S}=\mathcal{S} \circ \mathcal{R}(\varphi)$ for all $\varphi \in \mathbb{T}^{\nu}$.

Since in the complex coordinates $(z, \bar{z})$ the involution $\mathcal{S}$ defined in (2.4) reads as in (2.29), an operator $\mathbf{R}(\varphi)$ as in (3.15) is reversible, respectively anti-reversible, if, for any $i=1,2$,

$$
\mathcal{R}_{i}(-\varphi) \circ \mathcal{S}=-\mathcal{S} \circ \mathcal{R}_{i}(\varphi), \quad \text { resp. } \mathcal{R}_{i}(-\varphi) \circ \mathcal{S}=\mathcal{S} \circ \mathcal{R}_{i}(\varphi),
$$

where, with a small abuse of notation, we still denote $(\mathcal{S} u)(x)=\overline{u(-x)}$. Moreover, recalling that in the Fourier coordinates such involution reads as in (2.30), we obtain the following lemma.

Lemma 3.18. A $\varphi$-dependent family of operators $\mathbf{R}(\varphi), \varphi \in \mathbb{T}^{\nu}$, as in (3.15) is

- reversible if, for any $i=1,2$,

$$
\left(\mathcal{R}_{i}\right)_{j}^{j^{\prime}}(-\varphi)=-\overline{\left(\mathcal{R}_{i}\right)_{j}^{j^{\prime}}(\varphi)} \forall \varphi \in \mathbb{T}^{v} \text {, that is }\left(\mathcal{R}_{i}\right)_{j}^{j^{\prime}}(\ell)=-\overline{\left(\mathcal{R}_{i}\right)_{j}^{j^{\prime}}(\ell)} \quad \forall \ell \in \mathbb{Z}^{v} ;
$$

- reversibility preserving if, for any $i=1,2$,

$$
\left(\mathcal{R}_{i}\right)_{j}^{j^{\prime}}(-\varphi)=\overline{\left(\mathcal{R}_{i}\right)_{j}^{j^{\prime}}(\varphi)} \forall \varphi \in \mathbb{T}^{\nu} \text {, that is }\left(\mathcal{R}_{i}\right)_{j}^{j^{\prime}}(\ell)=\overline{\left(\mathcal{R}_{i}\right)_{j}^{j^{\prime}}(\ell)} \forall \ell \in \mathbb{Z}^{\nu}
$$

Note that the composition of a reversible operator with a reversibility preserving operator is reversible. The flow generated by a reversibility preserving operator is reversibility preserving. If $\mathcal{R}(\varphi)$ is reversibility preserving, then $\left(\omega \cdot \partial_{\varphi} \mathcal{R}\right)(\varphi)$ is reversible. 
We shall say that a linear operator of the form $\omega \cdot \partial_{\varphi}+A(\varphi)$ is reversible if $A(\varphi)$ is reversible. Conjugating the linear operator $\omega \cdot \partial_{\varphi}+A(\varphi)$ by a family of invertible linear maps $\Phi(\varphi)$, we get the transformed operator

$$
\begin{aligned}
& \Phi^{-1}(\varphi) \circ\left(\omega \cdot \partial_{\varphi}+A(\varphi)\right) \circ \Phi(\varphi)=\omega \cdot \partial_{\varphi}+A_{+}(\varphi), \\
& A_{+}(\varphi):=\Phi^{-1}(\varphi)\left(\omega \cdot \partial_{\varphi} \Phi(\varphi)\right)+\Phi^{-1}(\varphi) A(\varphi) \Phi(\varphi) .
\end{aligned}
$$

The conjugation of a reversible operator with a reversibility preserving operator is reversible.

Lemma 3.19. A pseudodifferential operator $\operatorname{Op}(a(\varphi, x, \xi))$ is reversible, respectively reversibility preserving, if and only if its symbol satisfies

$$
a(-\varphi,-x, \xi)=-\overline{a(\varphi, x, \xi)}, \quad \text { resp. } \quad a(-\varphi,-x, \xi)=\overline{a(\varphi, x, \xi)}
$$

Proof. If the symbols $a$ satisfies (3.47), then, recalling the complex form of the involution $\mathcal{S}$ in (2.29)-(2.30), we deduce that $\operatorname{Op}(a(\varphi, x, \xi))$ is reversible, respectively anti-reversible. The vice versa follows using that $a(\varphi, x, j)=e^{-\mathrm{i} j x}$ $\mathrm{Op}(a(\varphi, x, \xi))\left[e^{\mathrm{i} j x}\right]$.

Remark 3.20. Let $A(\varphi)=R(\varphi)+T(\varphi)$ be a reversible operator. Then $A(\varphi)=$ $R_{+}(\varphi)+T_{+}(\varphi)$ where both operators

$$
R_{+}(\varphi):=\frac{1}{2}(R(\varphi)-\mathcal{S} R(-\varphi) \mathcal{S}), \quad T_{+}(\varphi):=\frac{1}{2}(T(\varphi)-\mathcal{S} T(-\varphi) \mathcal{S}),
$$

are reversible. If $R(\varphi)=\mathrm{Op}(r(\varphi, x, \xi))$ is pseudodifferential, then

$$
R_{+}(\varphi)=\operatorname{Op}\left(r_{+}(\varphi, x, \xi)\right), \quad r_{+}(\varphi, x, \xi):=\frac{1}{2}(r(\varphi, x, \xi)-\overline{r(-\varphi,-x, \xi)})
$$

and the pseudodifferential norms of $\mathrm{Op}(r)$ and $\mathrm{Op}\left(r_{+}\right)$are equivalent. If $T(\varphi)$ is a tame operator with a tame constant $\mathfrak{M}_{T}(s)$, then $T_{+}(\varphi)$ is a tame operator as well with an equivalent tame constant.

Definition 3.21. (Reversible and anti-reversible function) A function $u(\varphi, \cdot)$ is called reversible if $\mathcal{S} u(\varphi, \cdot)=u(-\varphi, \cdot)$ (cfr. (2.17)), or is called anti-reversible if $-\mathcal{S} u(\varphi, \cdot)=u(-\varphi, \cdot)$. The same definition holds in the action-angle-normal variables $(\theta, I, w)$ with the involution $\overrightarrow{\mathcal{S}}$ defined in $(2.51)$ and in the $(z, \bar{z})$ complex variables with the involution in (2.29).

A reversibility preserving operator maps reversible, respectively anti-reversible, functions into reversible, respectively anti-reversible, functions.

Lemma 3.22. Let $X$ be a reversible vector field, according to (2.5), and $u(\varphi, x)$ be a reversible quasi-periodic function. Then the linearized operator $\mathrm{d}_{u} X(u(\varphi, \cdot))$ is reversible, according to Definition 3.17 .

Proof. Differentiating (2.5) we get $\left(\mathrm{d}_{u} X\right)(\mathcal{S} u) \circ \mathcal{S}=-\mathcal{S}\left(\mathrm{d}_{u} X\right)(u)$ and use $\mathcal{S} u(\varphi, \cdot)=u(-\varphi, \cdot)$.

Finally we note the following lemma: 
Lemma 3.23. The projections $\Pi_{\mathbb{S}^{+}, \Sigma}^{\top}, \Pi_{\mathbb{S}^{+}, \Sigma}^{L}$ defined in Section 2.3 commute with the involution $\mathcal{S}$ defined in (2.4), that is are reversibility preserving. The orthogonal projectors $\Pi_{\mathbb{S}}$ and $\Pi_{\mathbb{S}_{0}}^{\perp}$ commute with the involution in (2.29), that is are reversibility preserving.

Proof. The involution $\mathcal{S}$ defined in (2.4) maps $V_{n, \pm}$ into itself, acting as in (2.36). Then, by the decomposition (2.33), each projector $\Pi_{V_{n, \sigma}}$ commutes with $\mathcal{S}$.

\subsection{Momentum Preserving Operators}

The following definition is crucial in the construction of traveling waves.

Definition 3.24. (Momentum preserving) A $\varphi$-dependent family of linear operators $A(\varphi), \varphi \in \mathbb{T}^{\nu}$, is momentum preserving if

$$
A(\varphi-\vec{\jmath} \varsigma) \circ \tau_{\varsigma}=\tau_{\varsigma} \circ A(\varphi), \quad \forall \varphi \in \mathbb{T}^{\nu}, \varsigma \in \mathbb{R},
$$

where the translation operator $\tau_{\varsigma}$ is defined in (2.7). A linear matrix operator $\mathbf{A}(\varphi)$ of the form (3.14) or (3.15) is momentum preserving if each of its components is momentum preserving.

Momentum preserving operators are closed under several operations.

Lemma 3.25. Let $A(\varphi), B(\varphi)$ be momentum preserving operators. Then

(i) (Composition): $A(\varphi) \circ B(\varphi)$ is a momentum preserving operator.

(ii) (Adjoint): the adjoint $(A(\varphi))^{*}$ is momentum preserving.

(iii) (Inversion): If $A(\varphi)$ is invertible then $A(\varphi)^{-1}$ is momentum preserving.

(iv) (Flow): Assume that

$$
\partial_{t} \Phi^{t}(\varphi)=A(\varphi) \Phi^{t}(\varphi), \quad \Phi^{0}(\varphi)=\mathrm{Id},
$$

has a unique propagator $\Phi^{t}(\varphi)$ for any $t \in[0,1]$. Then $\Phi^{t}(\varphi)$ is momentum preserving.

Proof. Item $(i)$ follows directly by (3.48). Item ( $i i)$, respectively ( $i i i)$, follows by taking the adjoint, respectively the inverse, of (3.48) and using that $\tau_{\varsigma}^{*}=\tau_{-\varsigma}=$ $\tau_{\varsigma}^{-1}$. Finally, item (iv) holds because $\tau_{\varsigma}^{-1} \Phi^{t}(\varphi-\vec{J} \varsigma) \tau_{\varsigma}$ solves the same Cauchy problem in (3.49).

We shall say that a linear operator of the form $\omega \cdot \partial_{\varphi}+A(\varphi)$ is momentum preserving if $A(\varphi)$ is momentum preserving. In particular, conjugating a momentum preserving operator $\omega \cdot \partial_{\varphi}+A(\varphi)$ by a family of invertible linear momentum preserving maps $\Phi(\varphi)$, we obtain the transformed operator $\omega \cdot \partial_{\varphi}+A_{+}(\varphi)$ in (3.46) which is momentum preserving.

Lemma 3.26. Let $A(\varphi)$ be a momentum preserving linear operator and u a quasiperiodic traveling wave, according to Definition 3.1. Then $A(\varphi) u$ is a quasiperiodic traveling wave. 
Proof. It follows by Definition 3.24 and by the characterization of traveling waves in (3.2).

Lemma 3.27. Let $X$ be a vector field translation invariant, according to (2.8). Let $u$ be a quasi-periodic traveling wave. Then the linearized operator $\mathrm{d}_{u} X(u(\varphi, \cdot))$ is momentum preserving.

Proof. Differentiating (2.8) we get $\left(\mathrm{d}_{u} X\right)\left(\tau_{\varsigma} u\right) \circ \tau_{\varsigma}=\tau_{\varsigma}\left(\mathrm{d}_{u} X\right)(u), \varsigma \in \mathbb{R}$. Then, apply (3.2).

We now provide a characterization of the momentum preserving property in Fourier space.

Lemma 3.28. Let $\varphi$-dependent family of operators $A(\varphi), \varphi \in \mathbb{T}^{v}$, is momentum preserving if and only if the matrix elements of $A(\varphi)$, defined by (3.13), fulfill

$$
A_{j}^{j^{\prime}}(\ell) \neq 0 \Rightarrow \vec{J} \cdot \ell+j-j^{\prime}=0, \quad \forall \ell \in \mathbb{Z}^{v}, \quad j, j^{\prime} \in \mathbb{Z} .
$$

Proof. By (3.13) we have, for any function $u(x)$,

$$
\tau_{\varsigma}(A(\varphi) u)=\sum_{j, j^{\prime} \in \mathbb{Z}} \sum_{\ell \in \mathbb{Z}^{v}} A_{j}^{j^{\prime}}(\ell) e^{\mathrm{i} j \varsigma} u_{j^{\prime}} e^{\mathrm{i}(\ell \cdot \varphi+j x)}
$$

and

$$
A(\varphi-\vec{j} \varsigma)\left[\tau_{\varsigma} u\right]=\sum_{j, j^{\prime} \in \mathbb{Z}} \sum_{\ell \in \mathbb{Z}^{v}} A_{j}^{j^{\prime}}(\ell) e^{-\mathrm{i} \ell \cdot \vec{j} \varsigma} e^{\mathrm{i} j^{\prime} \varsigma} u_{j^{\prime}} e^{\mathrm{i}(\ell \cdot \varphi+j x)} .
$$

Therefore (3.48) is equivalent to (3.50).

We characterize the symbol of a pseudodifferential operator which is momentum preserving.

Lemma 3.29. A pseudodifferential operator $A(\varphi, x, D)=\operatorname{Op}(a(\varphi, x, \xi))$ is momentum preserving if and only if its symbol satisfies

$$
a(\varphi-\vec{\jmath} \varsigma, x, \xi)=a(\varphi, x+\varsigma, \xi), \quad \forall \varsigma \in \mathbb{R} .
$$

Proof. If the symbol $a$ satisfies (3.51), then, for all $\varsigma \in \mathbb{R}$,

$$
\tau_{\varsigma} \circ \mathrm{Op}(a(\varphi, x, \xi))=\mathrm{Op}(a(\varphi, x+\varsigma, \xi)) \circ \tau_{\varsigma}=\mathrm{Op}(a(\varphi-\vec{\jmath} \varsigma, x, \xi)) \circ \tau_{\varsigma},
$$

proving that $\tau_{\varsigma} \circ A(\varphi, x, D)=A(\varphi-\vec{\jmath} \varsigma, x, D) \circ \tau_{\varsigma}$. The vice versa follows using that $a(\varphi, x, \xi)=e^{-\mathrm{i} \xi x} A(\varphi, x, D)\left[e^{\mathrm{i} \xi x}\right]$.

Note that, if a symbol $a(\varphi, x, \xi)$ satisfies (3.51), then $\left(\omega \cdot \partial_{\varphi} a\right)(\varphi, x, \xi)$ satisfies (3.51) as well.

Lemma 3.30. If $\beta(\varphi, x)$ is a quasi-periodic traveling wave, then the operator $\mathcal{B}(\varphi)$ defined in (3.29) is momentum preserving. 
Proof. We have $\mathcal{B}(\varphi-\vec{\jmath} \varsigma)\left[\tau_{\varsigma} u\right]=u(x+\beta(\varphi-\vec{\jmath} \varsigma, x)+\varsigma)=u(x+\varsigma+\beta(\varphi, x+$ $\varsigma))=\tau_{\varsigma}(\mathcal{B}(\varphi) u)$.

We also note the following lemma:

Lemma 3.31. The symplectic projections $\Pi_{\mathbb{S}^{+}, \Sigma}^{\top}, \Pi_{\mathbb{S}^{+}, \Sigma}^{L}$, the $L^{2}$-projections $\Pi_{\angle}^{L^{2}}$ and $\Pi_{\mathbb{S}}, \Pi_{\mathbb{S}_{0}}^{\perp}$ defined in Section 2.3 commute with the translation operators $\tau_{\varsigma}$ defined in (2.7), that is are momentum preserving.

Proof. Recall that the translation $\tau_{\varsigma}$ maps $V_{n, \pm}$ into itself, acting as in (2.37). Consider the $L^{2}$-orthogonal decomposition $\mathfrak{H}=\mathfrak{H}_{\angle} \oplus \mathfrak{H}_{\angle}^{\perp}$, setting $\mathfrak{H}_{\angle}:=\mathfrak{H}_{\mathbb{S}^{+}, \Sigma}^{\angle}$ for brevity, to get

$$
u=\Pi_{\mathfrak{H}_{\angle}}^{L^{2}} u+\Pi_{\mathfrak{H}_{\perp}^{\perp}}^{L^{2}} u, \quad \Pi_{\mathfrak{H}_{\angle}}^{L^{2}} u \in \mathfrak{H}_{\angle}, \quad \Pi_{\mathfrak{H}_{\perp}^{\perp}}^{L^{2}} u \in \mathfrak{H}_{\angle}^{\perp} .
$$

Applying $\tau_{\varsigma}$ we get $\tau_{\varsigma} u=\tau_{\varsigma} \Pi_{\mathfrak{H}^{\prime}}^{L^{2}} u+\tau_{\zeta} \Pi_{\mathfrak{H}^{\perp}}^{L^{2}} u$. As shown above, $\tau_{\varsigma}$ maps $\mathfrak{H}_{\angle}$ into itself for all $\varsigma$. Thus also the $L^{2}$-orthogonal subspace $\mathfrak{H}_{\angle}^{\perp}$ is invariant under the action of $\tau_{\varsigma}$ and we conclude, by the uniqueness of the orthogonal decomposition, that $\tau_{\varsigma} \Pi_{\mathfrak{H}^{\prime}}^{L^{2}} u=\Pi_{\mathfrak{H}^{\prime}}^{L^{2}} \tau_{\varsigma} u, \tau_{\varsigma} \Pi_{\mathfrak{H}_{\llcorner}^{\perp}}^{L^{2}} u=\Pi_{\mathfrak{H}_{\llcorner}^{\perp}}^{L^{2}} \tau_{\zeta} u$.

The next lemma concerns the Dirichlet-Neumann operator.

Lemma 3.32. The Dirichlet-Neumann operator $G(\bar{\eta}, \mathrm{h})$, evaluated at a quasiperiodic traveling wave $\bar{\eta}(\varphi, x)$, is momentum preserving.

Proof. It follows by (2.9) and the characterization in (3.2) of the quasi-periodic traveling wave $\bar{\eta}(\varphi, x)$.

Quasi-periodic traveling waves in action-angle-normal coordinates. We now discuss how the momentum preserving condition reads in the coordinates $(\theta, I, w)$ introduced in (2.50). Recalling (2.52), if $u(\varphi, x)$ is a quasi-periodic traveling wave with action-angle-normal components $(\theta(\varphi), I(\varphi), w(\varphi, x))$, the condition $\tau_{\varsigma} u=$ $u(\varphi-\vec{\jmath} \varsigma, \cdot)$ becomes

$$
\left(\begin{array}{c}
\theta(\varphi)-\vec{\jmath} \varsigma \\
I(\varphi) \\
\tau_{\varsigma} w(\varphi, \cdot)
\end{array}\right)=\left(\begin{array}{c}
\theta(\varphi-\vec{\jmath} \varsigma) \\
I(\varphi-\vec{\jmath} \varsigma) \\
w(\varphi-\vec{\jmath} \varsigma, \cdot)
\end{array}\right), \quad \forall \varsigma \in \mathbb{R}
$$

As we look for $\theta(\varphi)$ of the form $\theta(\varphi)=\varphi+\Theta(\varphi)$, with a $(2 \pi)^{\nu}$-periodic function $\Theta: \mathbb{R}^{v} \mapsto \mathbb{R}^{v}, \varphi \mapsto \Theta(\varphi)$, the traveling wave condition becomes

$$
\left(\begin{array}{c}
\Theta(\varphi) \\
I(\varphi) \\
\tau_{\varsigma} w(\varphi, \cdot)
\end{array}\right)=\left(\begin{array}{c}
\Theta(\varphi-\vec{J} \varsigma) \\
I(\varphi-\vec{\jmath} \varsigma) \\
w(\varphi-\vec{\jmath} \varsigma, \cdot)
\end{array}\right), \quad \forall \varsigma \in \mathbb{R} .
$$


Definition 3.33. (Traveling wave variation) We call a traveling wave variation $g(\varphi)=\left(g_{1}(\varphi), g_{2}(\varphi), g_{3}(\varphi, \cdot)\right) \in \mathbb{R}^{v} \times \mathbb{R}^{v} \times \mathfrak{H}_{\mathbb{S}^{+}, \Sigma}^{L}$ a function satisfying (3.53), that is

$g_{1}(\varphi)=g_{1}(\varphi-\vec{\jmath} \varsigma), \quad g_{2}(\varphi)=g_{2}(\varphi-\vec{\jmath} \varsigma), \quad \tau_{\varsigma} g_{3}(\varphi)=g_{3}(\varphi-\vec{\jmath} \varsigma), \forall \varsigma \in \mathbb{R}$, or, equivalently, $D \vec{\tau}_{\varsigma} g(\varphi)=g(\varphi-\vec{\jmath} \varsigma)$ for any $\varsigma \in \mathbb{R}$, where $D \vec{\tau}_{\varsigma}$ is the differential of $\vec{\tau}_{\varsigma}$, namely

$$
D \vec{\tau}_{\varsigma}\left(\begin{array}{c}
\Theta \\
I \\
w
\end{array}\right)=\left(\begin{array}{c}
\Theta \\
I \\
\tau_{\varsigma} w
\end{array}\right), \quad \forall \varsigma \in \mathbb{R}
$$

According to Definition 3.24, a linear operator acting in $\mathbb{R}^{v} \times \mathbb{R}^{v} \times \mathfrak{H}_{\mathbb{S}^{+}, \Sigma}^{L}$ is momentum preserving if

$$
A(\varphi-\vec{\jmath} \varsigma) \circ D \vec{\tau}_{\varsigma}=D \vec{\tau}_{\varsigma} \circ A(\varphi), \quad \forall \varsigma \in \mathbb{R} .
$$

In a fashion similar to Lemma 3.26, one proves the following result:

Lemma 3.34. Let $A(\varphi)$ be a momentum preserving linear operator acting on $\mathbb{R}^{v} \times$ $\mathbb{R}^{v} \times \mathfrak{H}_{\mathbb{S}^{+}, \Sigma}^{L}$ and $g \in \mathbb{R}^{v} \times \mathbb{R}^{v} \times \mathfrak{H}_{\mathbb{S}^{+}, \Sigma}^{L}$ be a traveling wave variation. Then $A(\varphi) g(\varphi)$ is a traveling wave variation.

\section{Transversality of Linear Frequencies}

In this section we extend the KAM theory approach of $[3,6]$ in order to deal with the linear frequencies $\Omega_{j}(\kappa)$ defined in (1.11). The main novelty is the use of the momentum condition in the proof of Proposition 4.5. We shall also exploit that the tangential sites $\mathbb{S}:=\left\{\bar{J}_{1}, \ldots, \bar{J}_{\nu}\right\} \subset \mathbb{Z} \backslash\{0\}$ defined in (2.48), have all distinct modulus $\left|\bar{J}_{a}\right|=\bar{n}_{a}$, see assumption (1.15).

We first introduce the following definition:

Definition 4.1. A function $f=\left(f_{1}, \ldots, f_{N}\right):\left[\kappa_{1}, \kappa_{2}\right] \rightarrow \mathbb{R}^{N}$ is non-degenerate if, for any $c \in \mathbb{R}^{N} \backslash\{0\}$, the scalar function $f \cdot c$ is not identically zero on the whole interval $\left[\kappa_{1}, \kappa_{2}\right]$.

From a geometric point of view, if $f$ is non-degenerate it means that the image of the curve $f\left(\left[\kappa_{1}, \kappa_{2}\right]\right) \subset \mathbb{R}^{N}$ is not contained in any hyperplane of $\mathbb{R}^{N}$.

We shall use in the sequel that the maps $\kappa \mapsto \Omega_{j}(\kappa)$ are analytic in $\left[\kappa_{1}, \kappa_{2}\right]$. We decompose

$\Omega_{j}(\kappa)=\omega_{j}(\kappa)+\frac{\gamma}{2} \frac{G_{j}(0)}{j}, \quad \omega_{j}(\kappa):=\sqrt{\kappa G_{j}(0) j^{2}+g G_{j}(0)+\left(\frac{\gamma}{2} \frac{G_{j}(0)}{j}\right)^{2}}$.

Note that the dependence on $\kappa$ of $\Omega_{j}(\kappa)$ enters only through $\omega_{j}(\kappa)$, because $\frac{G_{j}(0)}{j}$ is independent of $\kappa$. Note also that $j \mapsto \omega_{j}(\kappa)$ is even in $j$, whereas the component due to the vorticity $j \mapsto \gamma \frac{G_{j}(0)}{j}$ is odd. Moreover this term is, in view of (1.8), uniformly bounded in $j$. 
Lemma 4.2. (Non-degeneracy-I) The following frequency vectors are non-degenerate:

1. $\vec{\Omega}(\kappa):=\left(\Omega_{j}(\kappa)\right)_{j \in \mathbb{S}} \in \mathbb{R}^{v}$;

2. $(\vec{\Omega}(\kappa), \sqrt{\kappa}) \in \mathbb{R}^{v+1}$;

3. $\left(\vec{\Omega}(\kappa), \Omega_{j}(\kappa)\right) \in \mathbb{R}^{\nu+1}$, for any $j \in \mathbb{Z} \backslash(\{0\} \cup \mathbb{S} \cup(-\mathbb{S}))$;

4. $\left(\vec{\Omega}(\kappa), \Omega_{j}(\kappa), \Omega_{j^{\prime}}(\kappa)\right) \in \mathbb{R}^{v+2}$, for any $j, j^{\prime} \in \mathbb{Z} \backslash(\{0\} \cup \mathbb{S} \cup(-\mathbb{S}))$ and $|j| \neq$ $\left|j^{\prime}\right|$.

Proof. Let

$$
\widetilde{\Omega}_{j}(\kappa):=\left\{\begin{array}{ll}
\Omega_{j}(\kappa) & \text { for } j \neq 0 \\
\sqrt{\kappa} & \text { for } j=0,
\end{array} \quad \widetilde{\omega}_{j}(\kappa):= \begin{cases}\omega_{j}(\kappa) & \text { for } j \neq 0 \\
\sqrt{\kappa} & \text { for } j=0 .\end{cases}\right.
$$

Recalling (4.1), we have that, for any $j \in \mathbb{Z}$,

$$
\partial_{\kappa} \widetilde{\omega}_{j}(\kappa)=\lambda_{j}(\kappa) \widetilde{\omega}_{j}(\kappa), \quad \lambda_{j}(\kappa):= \begin{cases}\frac{G_{j}(0) j^{2}}{2\left(\kappa G_{j}(0) j^{2}+g G_{j}(0)+\left(\frac{\gamma}{2} \frac{G_{j}(0)}{j}\right)^{2}\right)} & \text { for } j \neq 0 \\ \frac{1}{2 \kappa} & \text { for } j=0 .\end{cases}
$$

Moreover $\partial_{\kappa} \lambda_{j}(\kappa)=-2 \lambda_{j}(\kappa)^{2}$, for any $j \in \mathbb{Z}$, and therefore, for any $n \in \mathbb{N}$,

$$
\partial_{\kappa}^{n} \widetilde{\omega}_{j}(\kappa)=\widetilde{c}_{n} \lambda_{j}(\kappa)^{n} \widetilde{\omega}_{j}(\kappa), \quad \widetilde{c}_{n}:=c_{1} \cdot \ldots \cdot c_{n}, \quad c_{n}:=3-2 n .
$$

We now prove items 2 and 3, that is the non-degeneracy of the vector $\left(\vec{\Omega}(\kappa), \widetilde{\Omega}_{j}(\kappa)\right) \in$ $\mathbb{R}^{v+1}$ for any $j \in \mathbb{Z} \backslash(\mathbb{S} \cup(-\mathbb{S}))$, where $\widetilde{\Omega}_{j}(\kappa)$ is defined in (4.2). Items 1 and 4 follow similarly. For this purpose, by analyticity, it is sufficient to find one value of $\kappa \in\left[\kappa_{1}, \kappa_{2}\right]$ so that the determinant of the $(v+1) \times(v+1)$ matrix

$$
\mathcal{A}(\kappa):=\left(\begin{array}{cccc}
\partial_{\kappa} \Omega_{\bar{\jmath}_{1}}(\kappa) & \cdots & \partial_{\kappa} \Omega_{\bar{\jmath}_{\nu}}(\kappa) & \partial_{\kappa} \widetilde{\Omega}_{j}(\kappa) \\
\vdots & \ddots & \vdots & \vdots \\
\partial_{\kappa}^{\nu+1} \Omega_{\bar{\jmath}_{1}}(\kappa) & \cdots & \partial_{\kappa}^{\nu+1} \Omega_{\bar{\jmath}_{v}}(\kappa) & \partial_{\kappa}^{\nu+1} \widetilde{\widetilde{\Omega}}_{j}(\kappa)
\end{array}\right)
$$

is not zero. We actually show that $\operatorname{det} \mathcal{A}(\kappa) \neq 0$ for any $\kappa \in\left[\kappa_{1}, \kappa_{2}\right]$. By (4.2)-(4.4) and the multilinearity of the determinant function, we get

$$
\operatorname{det} \mathcal{A}(\kappa)=C(\kappa) \operatorname{det}\left(\begin{array}{cccc}
1 & \cdot & 1 & 1 \\
\lambda_{\bar{J}_{1}}(\kappa) & \cdot & \lambda_{\bar{J}_{v}}(\kappa) & \lambda_{j}(\kappa) \\
\vdots & \ddots & \vdots & \vdots \\
\lambda_{\bar{J}_{1}}(\kappa)^{\nu} & \cdot & \lambda_{\bar{J}_{v}}(\kappa)^{v} & \lambda_{j}(\kappa)^{v}
\end{array}\right)=: C(\kappa) \operatorname{det} \mathcal{B}(\kappa)
$$

where

$$
C(\kappa):=\prod_{q=1}^{\nu+1} \widetilde{c}_{q} \cdot \prod_{p \in\left\{\bar{J}_{1}, \ldots, \bar{J}_{\nu}, j\right\}} \lambda_{p}(\kappa) \widetilde{\omega}_{p}(\kappa) \neq 0, \quad \forall \kappa \in\left[\kappa_{1}, \kappa_{2}\right] .
$$

Since $\mathcal{B}(\kappa)$ is a Vandermorde matrix, we conclude that

$$
\operatorname{det} \mathcal{A}(\kappa)=C(\kappa) \prod_{p, p^{\prime} \in\left\{\bar{J}_{1}, \ldots, \bar{J}_{v}, j\right\}, p<p^{\prime}}\left(\lambda_{p}(\kappa)-\lambda_{p^{\prime}}(\kappa)\right) .
$$


Now, the fact that $\operatorname{det} \mathcal{A}(\kappa) \neq 0$ for any $\kappa \in\left[\kappa_{1}, \kappa_{2}\right]$ is a consequence from the following

Claim: For any $p, p^{\prime} \in\left\{\bar{J}_{1}, \ldots, \bar{J}_{\nu}, j\right\}, p \neq p^{\prime}$, one has $\lambda_{p}(\kappa) \neq \lambda_{p^{\prime}}(\kappa)$ for any $\kappa \in\left[\kappa_{1}, \kappa_{2}\right]$.

Proof of the Claim: If $p^{\prime}=0$ and $p \neq 0$, the claim follows because, by (4.3),

$$
\lambda_{p}(\kappa)=\frac{1}{2\left(\kappa+\frac{g}{p^{2}}+\frac{\gamma^{2}}{4} \frac{G_{p}(0)}{p^{4}}\right)}<\frac{1}{2 \kappa}=\lambda_{0}(\kappa)
$$

Consider now the case $p, p^{\prime} \neq 0$. We now prove that the map $p \mapsto \lambda_{p}(\kappa)$ is strictly monotone on $(0,+\infty)$. In case of finite depth, $G_{p}(0)=p \tanh (\mathrm{h} p)$, and

$$
\partial_{p} \lambda_{p}(\kappa)=\frac{1}{2\left(\kappa+\frac{g}{p^{2}}+\frac{\gamma^{2}}{4} \frac{\tanh (\mathrm{h} p)}{p^{3}}\right)^{2}}\left\{\frac{2 g}{p^{3}}+\frac{\gamma^{2}}{4} \frac{3 \tanh (\mathrm{h} p)-\left(1-\tanh ^{2}(\mathrm{~h} p)\right) \mathrm{h} p}{p^{4}}\right\} .
$$

The function $f(y):=3 \tanh (y)-\left(1-\tanh ^{2}(y)\right) y$ is positive for any $y>0$. Indeed $f(y) \rightarrow 0$ as $y \rightarrow 0$, and it is strictly monotone increasing for $y>0$, since $f^{\prime}(y)=2\left(1-\tanh ^{2}(y)\right)(1+y \tanh (y))>0$. We deduce that $\partial_{p} \lambda_{p}(\kappa)>0$, also if the depth $h=+\infty$. Since the function $p \mapsto \lambda_{p}(\kappa)$ is even we have proved that that it is strictly monotone decreasing on $(-\infty, 0)$ and increasing in $(0,+\infty)$. Thus, if $\lambda_{p}(\kappa)=\lambda_{p^{\prime}}(\kappa)$ then $p=-p^{\prime}$. But this case is excluded by the assumption (1.15) and the condition $j \notin \mathbb{S} \cup(-\mathbb{S})$, which together imply $|p| \neq\left|p^{\prime}\right|$.

Note that in items 3 and 4 of Lemma 4.2 we require that $j$ and $j^{\prime}$ do not belong to $\{0\} \cup \mathbb{S} \cup(-\mathbb{S})$. In order to deal in Proposition 4.5 when $j$ and $j^{\prime}$ are in $\mathbb{S} \cup(-\mathbb{S})$, we need also the following lemma. It is actually a direct consequence of the proof of Lemma 4.2, noting that $\Omega_{j}(\kappa)-\omega_{j}(\kappa)$ is independent of $\kappa$.

Lemma 4.3. (Non-degeneracy-II) Let $\vec{\omega}(\kappa):=\left(\omega_{\bar{\jmath}_{1}}(\kappa), \ldots, \omega_{\bar{J}_{\nu}}(\kappa)\right)$. The following vectors are non-degenerate:

1. $(\vec{\omega}(\kappa), 1) \in \mathbb{R}^{\nu+1}$;

2. $\left(\vec{\omega}(\kappa), \omega_{j}(\kappa), 1\right) \in \mathbb{R}^{v+2}$, for any $j \in \mathbb{Z} \backslash(\{0\} \cup \mathbb{S} \cup(-\mathbb{S}))$.

For later use, we provide the following asymptotic estimate of the linear frequencies:

Lemma 4.4. (Asymptotics) For any $j \in \mathbb{Z} \backslash\{0\}$, we have

$$
\omega_{j}(\kappa)=\sqrt{\kappa}|j|^{\frac{3}{2}}+\frac{c_{j}(\kappa)}{\sqrt{\kappa}|j|^{\frac{1}{2}}},
$$

where, for any $n \in \mathbb{N}_{0}$, there exists a constant $C_{n, \mathrm{~h}}>0$ such that

$$
\sup _{\substack{j \in \mathbb{Z} \backslash\{0\} \\ \kappa \in\left[\kappa_{1}, \kappa_{2}\right]}}\left|\partial_{\kappa}^{n} \frac{c_{j}(\kappa)}{\sqrt{\kappa}}\right| \leqq C_{n, \mathrm{~h}}
$$


Proof. By (4.1) we deduce (4.5) with

$$
c_{j}(\kappa):=\frac{\kappa|j|\left(G_{j}(0)-|j|\right)+\frac{g G_{j}(0)}{|j|}\left(1+\left(\frac{\gamma}{2}\right)^{2} \frac{G_{j}(0)}{g|j|^{2}}\right)}{1+\sqrt{1+\frac{G_{j}(0)-|j|}{|j|}+\frac{g G_{j}(0)}{\kappa|j|^{3}}\left(1+\left(\frac{\gamma}{2}\right)^{2} \frac{G_{j}(0)}{g|j|^{2}}\right)}} .
$$

Then (4.6) follows exploiting that (both for finite and infinite depth) the quantities $|j|\left(G_{j}(0)-|j|\right)$ and $G_{j}(0) /|j|$ are uniformly bounded in $j$, see (1.8).

The next proposition is the key of the argument. We remind that $\vec{J}=\left(\bar{J}_{1}, \ldots, \bar{J}_{v}\right)$ denotes the vector in $\mathbb{Z}^{v}$ of tangential sites introduced in (2.53).

Proposition 4.5. (Transversality) There exist $m_{0} \in \mathbb{N}$ and $\rho_{0}>0$ such that, for any $\kappa \in\left[\kappa_{1}, \kappa_{2}\right]$, the following hold:

$$
\begin{aligned}
& \max _{0 \leqq n \leqq m_{0}}\left|\partial_{\kappa}^{n} \vec{\Omega}(\kappa) \cdot \ell\right| \geqq \rho_{0}\langle\ell\rangle, \quad \forall \ell \in \mathbb{Z}^{v} \backslash\{0\} ; \\
& \left\{\begin{array}{l}
\max _{0 \leqq n \leqq m_{0}}\left|\partial_{\kappa}^{n}\left(\vec{\Omega}(\kappa) \cdot \ell+\Omega_{j}(\kappa)\right)\right| \geqq \rho_{0}\langle\ell\rangle \\
\vec{J} \cdot \ell+j=0, \quad \ell \in \mathbb{Z}^{v}, j \in \mathbb{S}_{0}^{c}
\end{array}\right. \\
& \left\{\begin{array}{l}
\max _{0 \leqq n \leqq m_{0}}\left|\partial_{\kappa}^{n}\left(\vec{\Omega}(\kappa) \cdot \ell+\Omega_{j}(\kappa)-\Omega_{j^{\prime}}(\kappa)\right)\right| \geqq \rho_{0}\langle\ell\rangle \\
\vec{J} \cdot \ell+j-j^{\prime}=0, \quad \ell \in \mathbb{Z}^{v}, j, j^{\prime} \in \mathbb{S}_{0}^{c},\left(\ell, j, j^{\prime}\right) \neq(0, j, j) ;
\end{array}\right. \\
& \left\{\begin{array}{l}
\max _{0 \leqq n \leqq m_{0}}\left|\partial_{\kappa}^{n}\left(\vec{\Omega}(\kappa) \cdot \ell+\Omega_{j}(\kappa)+\Omega_{j^{\prime}}(\kappa)\right)\right| \geqq \rho_{0}\langle\ell\rangle \\
\vec{J} \cdot \ell+j+j^{\prime}=0, \ell \in \mathbb{Z}^{v}, j, j^{\prime} \in \mathbb{S}_{0}^{c} .
\end{array}\right.
\end{aligned}
$$

We call $\rho_{0}$ the amount of non-degeneracy and $m_{0}$ the index of non-degeneracy.

Proof. We prove separately (4.7)-(4.10). In this proof we set for brevity $\mathfrak{K}:=$ $\left[\kappa_{1}, \kappa_{2}\right]$.

Proof of (4.7). By contradiction, assume that for any $m \in \mathbb{N}$ there exist $\kappa_{m} \in \mathfrak{K}$ and $\ell_{m} \in \mathbb{Z}^{v} \backslash\{0\}$ such that

$$
\left|\partial_{\kappa}^{n} \vec{\Omega}\left(\kappa_{m}\right) \cdot \frac{\ell_{m}}{\left\langle\ell_{m}\right\rangle}\right|<\frac{1}{\langle m\rangle}, \quad \forall 0 \leqq n \leqq m
$$

The sequences $\left(\kappa_{m}\right)_{m \in \mathbb{N}} \subset \mathfrak{K}$ and $\left(\ell_{m} /\left\langle\ell_{m}\right\rangle\right)_{m \in \mathbb{N}} \subset \mathbb{R}^{v} \backslash\{0\}$ are both bounded. By compactness, up to subsequences $\kappa_{m} \rightarrow \bar{\kappa} \in \mathfrak{K}$ and $\ell_{m} /\left\langle\ell_{m}\right\rangle \rightarrow \bar{c} \neq 0$. Therefore, in the limit for $m \rightarrow+\infty$, by (4.11) we get $\partial_{\kappa}^{n} \vec{\Omega}(\bar{\kappa}) \cdot \bar{c}=0$ for any $n \in \mathbb{N}_{0}$. By the analyticity of $\vec{\Omega}(\kappa)$, we deduce that the function $\kappa \mapsto \vec{\Omega}(\kappa) \cdot \bar{c}$ is identically zero on $\mathfrak{K}$, which contradicts Lemma 4.2-1.

Proof of (4.8). We divide the proof in 4 steps.

STEP I. Recalling (4.1) and Lemma 4.4, we have that, for any $\kappa \in \mathfrak{K}$,

$$
\left|\vec{\Omega}(\kappa) \cdot \ell+\Omega_{j}(\kappa)\right| \geqq\left|\Omega_{j}(\kappa)\right|-|\vec{\Omega}(\kappa) \cdot \ell| \geqq \sqrt{\kappa_{1}}|j|^{\frac{3}{2}}-C\langle\ell\rangle \geqq\langle\ell\rangle
$$


whenever $|j|^{\frac{3}{2}} \geqq C_{0}\langle\ell\rangle$, for some $C_{0}>0$. In this cases (4.8) is already fulfilled with $n=0$. Hence we restrict in the sequel to indexes $\ell \in \mathbb{Z}^{v}$ and $j \in \mathbb{S}_{0}^{c}$ satisfying

$$
|j|^{\frac{3}{2}}<C_{0}\langle\ell\rangle
$$

STEP 2. By contradiction, we assume that, for any $m \in \mathbb{N}$, there exist $\kappa_{m} \in \mathfrak{K}$, $\ell_{m} \in \mathbb{Z}^{v}$ and $j_{m} \in \mathbb{S}_{0}^{c}$, with $\left|j_{m}\right|^{\frac{3}{2}}<C_{0}\left\langle\ell_{m}\right\rangle$, such that, for any $n \in \mathbb{N}_{0}$ with $n \leqq m$,

$$
\left\{\begin{array}{l}
\left|\partial_{\kappa}^{n}\left(\vec{\Omega}(\kappa) \cdot \frac{\ell_{m}}{\left\langle\ell_{m}\right\rangle}+\frac{1}{\left\langle\ell_{m}\right\rangle} \Omega_{j_{m}}(\kappa)\right)_{\mid \kappa=\kappa_{m}}\right|<\frac{1}{\langle m\rangle} \\
\vec{J} \cdot \ell_{m}+j_{m}=0
\end{array}\right.
$$

Up to subsequences $\kappa_{m} \rightarrow \bar{\kappa} \in \mathfrak{K}$ and $\ell_{m} /\left\langle\ell_{m}\right\rangle \rightarrow \bar{c} \in \mathbb{R}^{v}$.

STEP 3. We consider first the case when the sequence $\left(\ell_{m}\right)_{m \in \mathbb{N}} \subset \mathbb{Z}^{v}$ is bounded. Up to subsequences, we have definitively that $\ell_{m}=\bar{\ell} \in \mathbb{Z}^{v}$. Moreover, since $j_{m}$ and $\ell_{m}$ satisfy (4.12), also the sequence $\left(j_{m}\right)_{m \in \mathbb{N}}$ is bounded and, up to subsequences, definitively $j_{m}=\bar{\jmath} \in \mathbb{S}_{0}^{c}$. Therefore, in the limit $m \rightarrow \infty$, from (4.13) we obtain

$$
\partial_{\kappa}^{n}\left(\vec{\Omega}(\kappa) \cdot \bar{\ell}+\Omega_{\bar{J}}(\kappa)\right)_{\mid \kappa=\bar{\kappa}}=0, \forall n \in \mathbb{N}_{0}, \quad \vec{\jmath} \cdot \bar{\ell}+\bar{\jmath}=0 .
$$

By analyticity, this implies

$$
\vec{\Omega}(\kappa) \cdot \bar{\ell}+\Omega_{\bar{J}}(\kappa)=0, \forall \kappa \in \mathfrak{K}, \quad \vec{J} \cdot \bar{\ell}+\bar{J}=0 .
$$

We distinguish two cases:

- Let $\bar{J} \notin-\mathbb{S}$. By (4.14) the vector $\left(\vec{\Omega}(\kappa), \Omega_{\bar{J}}(\kappa)\right)$ is degenerate according to Definition 4.1 with $c:=(\bar{\ell}, 1) \neq 0$. This contradicts Lemma 4.2-3.

- Let $\bar{j} \in-\mathbb{S}$. With no loss of generality suppose $\bar{j}=-\bar{J}_{1}$. Then, denoting $\bar{\ell}=\left(\overline{\ell_{1}}, \ldots, \overline{\ell_{v}}\right)$, system (4.14) reads, for any $\kappa \in \mathfrak{K}$,

$$
\left\{\begin{array}{l}
\left(\overline{\ell_{1}}+1\right) \omega_{\bar{J}_{1}}(\kappa)+\sum_{a=2}^{v} \overline{\ell_{a}} \omega_{\bar{J}_{a}}(\kappa)+\frac{\gamma}{2}\left(\left(\overline{\ell_{1}}-1\right) \frac{G_{\bar{J}_{1}}(0)}{\bar{J}_{1}}+\sum_{a=2}^{v} \overline{\ell_{a}} \frac{G_{\bar{J}_{a}}(0)}{\bar{J}_{a}}\right)=0 \\
\left(\overline{\ell_{1}}-1\right) \bar{J}_{1}+\sum_{a=2}^{v} \overline{\ell_{a}} \bar{J}_{a}=0 .
\end{array}\right.
$$

By Lemma 4.3-1 the vector $(\vec{\omega}(\kappa), 1)$ is non-degenerate, which is a contradiction for $\gamma \neq 0$. If $\gamma=0$ we only deduce $\overline{\ell_{1}}=-1$ and $\overline{\ell_{2}}=\ldots=\overline{\ell_{v}}=0$. Inserting these values in the momentum condition in (4.15), we get $2 \bar{J}_{1}=0$. This is a contradiction with $\bar{J}_{1} \neq 0$.

STEP 4. We consider now the case when the sequence $\left(\ell_{m}\right)_{m \in \mathbb{N}}$ is unbounded. Up to subsequences $\left|\ell_{m}\right| \rightarrow \infty$ as $m \rightarrow \infty$ and $\lim _{m \rightarrow \infty} \ell_{m} /\left\langle\ell_{m}\right\rangle=: \bar{c} \neq 0$. By (4.1) and (4.5), for any $n \in \mathbb{N}_{0}$,

$$
\begin{aligned}
\partial_{\kappa}^{n} \frac{1}{\left\langle\ell_{m}\right\rangle} \Omega_{j_{m}}\left(\kappa_{m}\right) & =\partial_{\kappa}^{n}\left(\frac{1}{\left\langle\ell_{m}\right\rangle} \sqrt{\kappa}\left|j_{m}\right|^{\frac{3}{2}}+\frac{c_{j_{m}}(\kappa)}{\left\langle\ell_{m}\right\rangle \sqrt{\kappa}\left|j_{m}\right|^{\frac{1}{2}}}+\frac{\gamma}{2\left\langle\ell_{m}\right\rangle} \frac{G_{j_{m}}(0)}{j_{m}}\right)_{\mid \kappa=\kappa_{m}} \\
& \stackrel{(4.6)}{\rightarrow} \bar{d}\left(\partial_{\kappa}^{n} \sqrt{\kappa}\right)_{\mid \kappa=\bar{\kappa}}, \text { for } m \rightarrow \infty,
\end{aligned}
$$


with $\bar{d}:=\lim _{m \rightarrow \infty}\left|j_{m}\right|^{\frac{3}{2}} /\left\langle\ell_{m}\right\rangle \in \mathbb{R}$. Note that $\bar{d}$ is finite because $j_{m}$ and $\ell_{m}$ satisfy (4.12). Therefore (4.13) becomes, in the limit $m \rightarrow \infty$,

$$
\partial_{\kappa}^{n}(\vec{\Omega}(\kappa) \cdot \bar{c}+\bar{d} \sqrt{\kappa})_{\mid \kappa=\bar{\kappa}}=0, \quad \forall n \in \mathbb{N}_{0} .
$$

By analyticity, this implies that $\vec{\Omega}(\kappa) \cdot \bar{c}+\bar{d} \sqrt{\kappa}=0$ for any $\kappa \in \mathfrak{K}$. This contradicts the non-degeneracy of the vector $(\vec{\Omega}(\kappa), \sqrt{\kappa})$ in Lemma $4.2-2$, since $(\bar{c}, \bar{d}) \neq 0$.

Proof of (4.9). We split again the proof into 4 steps.

STEP I. By Lemma 4.4, for any $\kappa \in \mathfrak{K}$,

$$
\begin{aligned}
\left|\vec{\Omega}(\kappa) \cdot \ell+\Omega_{j}(\kappa)-\Omega_{j^{\prime}}(\kappa)\right| & \geqq\left|\Omega_{j}(\kappa)-\Omega_{j^{\prime}}(\kappa)\right|-|\vec{\Omega}(\kappa) \cdot \ell| \\
& \geqq\left.\sqrt{\kappa}|| j\right|^{\frac{3}{2}}-\left|j^{\prime}\right|^{\frac{3}{2}} \mid-C\langle\ell\rangle \geqq\langle\ell\rangle
\end{aligned}
$$

whenever $\left.|| j\right|^{\frac{3}{2}}-\left|j^{\prime}\right|^{\frac{3}{2}} \mid \geqq C_{1}\langle\ell\rangle$ for some $C_{1}>0$. In this case (4.10) is already fulfilled with $n=0$. Thus we restrict to indexes $\ell \in \mathbb{Z}^{v}$ and $j, j^{\prime} \in \mathbb{S}_{0}^{c}$, such that

$$
\left.|| j\right|^{\frac{3}{2}}-\left|j^{\prime}\right|^{\frac{3}{2}} \mid<C_{1}\langle\ell\rangle \text {. }
$$

Furthermore we may assume $j_{m} \neq j_{m}^{\prime}$ because the case $j_{m}=j_{m}^{\prime}$ is included in (4.7).

STEP 2. By contradiction, we assume that, for any $m \in \mathbb{N}$, there exist $\kappa_{m} \in \mathfrak{K}$, $\ell_{m} \in \mathbb{Z}^{v}$ and $j_{m}, j_{m}^{\prime} \in \mathbb{S}_{0}^{c}$, satisfying (4.16), such that, for any $0 \leqq n \leqq m$,

$$
\left\{\begin{array}{l}
\left|\partial_{\kappa}^{n}\left(\vec{\Omega}(\kappa) \cdot \frac{\ell_{m}}{\left\langle\ell_{m}\right\rangle}+\frac{1}{\left\langle\ell_{m}\right\rangle}\left(\Omega_{j_{m}}(\kappa)-\Omega_{j_{m}^{\prime}}(\kappa)\right)\right)_{\mid \kappa=\kappa_{m}}\right|<\frac{1}{\langle m\rangle} \\
\vec{J} \cdot \ell_{m}+j_{m}-j_{m}^{\prime}=0
\end{array}\right.
$$

Up to subsequences $\kappa_{m} \rightarrow \bar{\kappa} \in \mathfrak{K}$ and $\ell_{m} /\left\langle\ell_{m}\right\rangle \rightarrow \bar{c} \in \mathbb{R}^{v}$.

STEP 3. We start with the case when $\left(\ell_{m}\right)_{m \in \mathbb{N}} \subset \mathbb{Z}^{v}$ is bounded. Up to subsequences, we have definitively that $\ell_{m}=\bar{\ell} \in \mathbb{Z}^{v}$. Moreover, if $\left|j_{m}\right| \neq\left|j_{m}^{\prime}\right|$, there is $c>0$ such that

$$
c\left(\left|j_{m}\right|^{\frac{1}{2}}+\left|j_{m}^{\prime}\right|^{\frac{1}{2}}\right) \leqq\left.|| j_{m}\right|^{\frac{3}{2}}-\left|j_{m}^{\prime}\right|^{\frac{3}{2}} \mid<C_{1}\left\langle\ell_{m}\right\rangle \leqq C, \quad \forall m \in \mathbb{N},
$$

If $j_{m}=-j_{m}^{\prime}$ we deduce by the momentum relation that $\left|j_{m}\right|=\left|j_{m}^{\prime}\right| \leqq C\left\langle\ell_{m}\right\rangle \leqq C$, and we conclude that in any case the sequences $\left(j_{m}\right)_{m \in \mathbb{N}}$ and $\left(j_{m}^{\prime}\right)_{m \in \mathbb{N}}$ are bounded. Up to subsequences, we have definitively that $j_{m}=\bar{\jmath}$ and $j_{m}^{\prime}=\bar{\jmath}^{\prime}$, with $\bar{\jmath}, \bar{J}^{\prime} \in \mathbb{S}_{0}^{c}$ and such that

$$
\bar{J} \neq \bar{J}^{\prime} .
$$

Therefore (4.17) becomes, in the limit $m \rightarrow \infty$,

$$
\partial_{\kappa}^{n}\left(\vec{\Omega}(\kappa) \cdot \bar{\ell}+\Omega_{\bar{\jmath}}(\kappa)-\Omega_{\bar{J}^{\prime}}(\kappa)\right)_{\mid \kappa=\bar{\kappa}}=0, \forall n \in \mathbb{N}_{0}, \quad \vec{J} \cdot \bar{\ell}+\bar{J}-\bar{J}^{\prime}=0 .
$$

By analyticity, we obtain that

$$
\vec{\Omega}(\kappa) \cdot \bar{\ell}+\Omega_{\bar{J}}(\kappa)-\Omega_{\bar{\jmath}^{\prime}}(\kappa)=0, \forall \kappa \in \mathfrak{K}, \quad \vec{j} \cdot \bar{\ell}+\bar{J}-\bar{J}^{\prime}=0 .
$$

We distinguish several cases: 
- Let $\bar{J}, \bar{J}^{\prime} \notin-\mathbb{S}$ and $|\bar{J}| \neq\left|\bar{J}^{\prime}\right|$. By (4.19) the vector $\left(\vec{\Omega}(\kappa), \Omega_{\bar{J}}(\kappa), \Omega_{\bar{J}^{\prime}}(\kappa)\right)$ is degenerate with $c:=(\bar{\ell}, 1,-1) \neq 0$, contradicting Lemma 4.2-4.

- Let $\bar{J}, \bar{J}^{\prime} \notin-\mathbb{S}$ and $\bar{J}^{\prime}=-\bar{\jmath}$. In view of (4.1), system (4.19) becomes

$$
\left\{\begin{array}{l}
\vec{\omega}(\kappa) \cdot \bar{\ell}+\frac{\gamma}{2}\left(\sum_{a=1}^{v} \overline{\ell_{a}} \frac{G_{\bar{J}_{a}}(0)}{\bar{J}_{a}}+2 \frac{G_{\bar{J}}(0)}{\bar{J}}\right)=0, \quad \forall \kappa \in \mathfrak{K}, \\
\vec{\jmath} \cdot \bar{\ell}+2 \bar{J}=0 .
\end{array}\right.
$$

By Lemma $4.3-1$, the vector $(\vec{\omega}(\kappa), 1)$ is non-degenerate, which is a contradiction for $\gamma \neq 0$. If $\gamma=0$ the first equation in (4.20) implies $\bar{\ell}=0$. Then the momentum condition implies $2 \bar{\jmath}=0$, which is a contradiction with $\bar{\jmath} \neq 0$.

- Let $\bar{\jmath}^{\prime} \notin-\mathbb{S}$ and $\bar{\jmath} \in-\mathbb{S}$. With no loss of generality suppose $\bar{\jmath}=-\bar{J}_{1}$. In view of (4.1), the first equation in (4.19) implies that, for any $\kappa \in \mathfrak{K}$,

$$
\begin{aligned}
& \left(\overline{\ell_{1}}+1\right) \omega_{\bar{J}_{1}}(\kappa)+\sum_{a=2}^{v} \overline{\ell_{a}} \omega_{\bar{J}_{a}}(\kappa)-\omega_{\bar{J}^{\prime}}(\kappa) \\
& \quad+\frac{\gamma}{2}\left(\left(\overline{\ell_{1}}-1\right) \frac{G_{\bar{\jmath}_{1}}(0)}{\bar{J}_{1}}+\sum_{a=2}^{v} \overline{\ell_{a}} \frac{G_{\bar{J}_{a}}(0)}{\bar{J}_{a}}-\frac{G_{\bar{J}^{\prime}}(0)}{\bar{J}^{\prime}}\right)=0 .
\end{aligned}
$$

By Lemma $4.3-2$, the vector $\left(\vec{\omega}(\kappa), \omega_{\bar{\jmath}^{\prime}}(\kappa), 1\right)$ is non-degenerate, which is a contradiction.

- Last, let $\bar{J}, \bar{J}^{\prime} \in-\mathbb{S}$ and $\bar{J} \neq \bar{J}^{\prime}$, by (4.18). With no loss of generality suppose $\bar{J}=-\bar{J}_{1}$ and $\bar{J}^{\prime}=-\bar{J}_{2}$. Then (4.19) reads, for any $\kappa \in \mathfrak{K}$,

$$
\left\{\begin{array}{l}
\left(\overline{\ell_{1}}+1\right) \omega_{\bar{J}_{1}}(\kappa)+\left(\overline{\ell_{2}}-1\right) \omega_{\bar{J}_{2}}+\sum_{a=3}^{v} \overline{\ell_{a}} \omega_{\bar{J}_{a}}(\kappa) \\
\left.+\frac{\gamma}{2}\left(\left(\overline{\ell_{1}}-1\right) \frac{G_{\bar{J}_{1}}(0)}{\bar{J}_{1}}+\left(\overline{\ell_{2}}+1\right) \frac{G_{\bar{J}_{2}}(0)}{\bar{J}_{2}}+\sum_{a=3}^{v} \overline{\ell_{a}} \frac{G_{\bar{J}_{a}}(0)}{\bar{J}_{a}}\right)\right)=0, \\
\left(\overline{\ell_{1}}-1\right) \bar{J}_{1}+\left(\overline{\ell_{2}}+1\right) \bar{J}_{2}+\sum_{a=3}^{v} \overline{\ell_{a}} \bar{J}_{a}=0 .
\end{array}\right.
$$

By Lemma $4.3-1$, the vector $(\vec{\omega}(\kappa), 1)$ is non-degenerate, therefore the first equation in (4.21) can hold only if $\overline{\ell_{1}}=-1, \overline{\ell_{2}}=1, \overline{\ell_{3}}=\ldots=\overline{\ell_{v}}=0$. Inserting these values in the momentum condition we obtain $-2 \bar{\jmath}_{1}+2 \bar{J}_{2}=0$. This contradicts $\bar{J} \neq \bar{J}^{\prime}$.

STEP 4 . We finally consider the case when $\left(\ell_{m}\right)_{m \in \mathbb{N}}$ is unbounded. Up to subsequences $\left|\ell_{m}\right| \rightarrow \infty$ as $m \rightarrow \infty$ and $\lim _{m \rightarrow \infty} \ell_{m} /\left\langle\ell_{m}\right\rangle=: \bar{c} \neq 0$. In addition, by (4.16), up to subsequences

$$
\lim _{m \rightarrow \infty} \frac{\left|j_{m}\right|^{\frac{3}{2}}-\left|j_{m}^{\prime}\right|^{\frac{3}{2}}}{\left\langle\ell_{m}\right\rangle}=\bar{d}_{1} \in \mathbb{R}
$$

By (4.1) and (4.5) we have, for any $n$,

$$
\begin{aligned}
& \partial_{\kappa}^{n} \frac{1}{\left\langle\ell_{m}\right\rangle}\left(\Omega_{j_{m}}(\kappa)-\Omega_{j_{m}^{\prime}}(\kappa)\right)_{\mid \kappa=\kappa_{m}}=\partial_{\kappa}^{n}\left(\frac{\sqrt{\kappa}}{\left\langle\ell_{m}\right\rangle}\left(\left|j_{m}\right|^{\frac{3}{2}}-\left|j_{m}^{\prime}\right|^{\frac{3}{2}}\right)\right. \\
& \left.\quad+\frac{1}{\left\langle\ell_{m}\right\rangle \sqrt{\kappa}}\left(\frac{c_{j_{m}}(\kappa)}{\left|j_{m}\right|^{\frac{1}{2}}}-\frac{c_{j_{m}^{\prime}}(\kappa)}{\left|j_{m}^{\prime}\right|^{\frac{1}{2}}}\right)+\frac{\gamma}{2\left\langle\ell_{m}\right\rangle}\left(\frac{G_{j_{m}}(0)}{j_{m}}-\frac{G_{j_{m}^{\prime}}(0)}{j_{m}^{\prime}}\right)_{\mid \kappa=\kappa_{m}}\right)
\end{aligned}
$$


$\rightarrow \bar{d}_{1} \partial_{\kappa}^{n}(\sqrt{\kappa})_{\mid \kappa=\bar{\kappa}}$ for $m \rightarrow \infty$,

using (4.22) and $\left\langle\ell_{m}\right\rangle \rightarrow \infty$. Therefore (4.17) becomes, in the limit $m \rightarrow \infty$,

$$
\partial_{\kappa}^{n}\left(\vec{\Omega}(\kappa) \cdot \bar{c}+\bar{d}_{1} \sqrt{\kappa}\right)_{\mid \kappa=\bar{\kappa}}=0, \quad \forall n \in \mathbb{N}_{0} .
$$

By analyticity this implies $\vec{\Omega}(\kappa) \cdot \bar{c}+\bar{d}_{1} \sqrt{\kappa}=0$, for all $\kappa \in \mathfrak{K}$. Thus $(\vec{\Omega}(\kappa), \sqrt{\kappa})$ is degenerate with $c=\left(\bar{c}, \bar{d}_{1}\right) \neq 0$, contradicting Lemma 4.2-2.

Proof of (4.10). The proof is similar to that for (4.9) and we omit it.

\section{Nash-Moser Theorem and Measure Estimates}

Under the rescaling $(\eta, \zeta) \mapsto(\varepsilon \eta, \varepsilon \zeta)$, the Hamiltonian system (2.14) transforms into the Hamiltonian system generated by

$$
\mathcal{H}_{\varepsilon}(\eta, \zeta):=\varepsilon^{-2} \mathcal{H}(\varepsilon \eta, \varepsilon \zeta)=\mathcal{H}_{L}(\eta, \zeta)+\varepsilon P_{\varepsilon}(\eta, \zeta),
$$

where $\mathcal{H}$ is the water waves Hamiltonian (2.13) expressed in the Wahlén coordinates (2.11), $\mathcal{H}_{L}$ is defined in (2.20) and

$$
\begin{aligned}
& P_{\varepsilon}(\eta, \zeta):=\frac{1}{2 \varepsilon} \int_{\mathbb{T}}\left(\zeta+\frac{\gamma}{2} \partial_{x}^{-1} \eta\right)(G(\varepsilon \eta)-G(0))\left(\zeta+\frac{\gamma}{2} \partial_{x}^{-1} \eta\right) \mathrm{d} x \\
& \quad+\frac{\kappa}{\varepsilon^{3}} \int_{\mathbb{T}}\left(\sqrt{1+\varepsilon^{2} \eta_{x}^{2}}-1-\frac{\varepsilon^{2} \eta_{x}^{2}}{2}\right) \mathrm{d} x+\frac{\gamma}{2} \int_{\mathbb{T}}\left(-\left(\zeta+\frac{\gamma}{2} \partial_{x}^{-1} \eta\right)_{x} \eta^{2}+\frac{\gamma}{3} \eta^{3}\right) \mathrm{d} x .
\end{aligned}
$$

We now study the Hamiltonian system generated by the Hamiltonian $\mathcal{H}_{\varepsilon}(\eta, \zeta)$, in the action-angle and normal coordinates $(\theta, I, w)$ defined in Section 2.3. Thus we consider the Hamiltonian $H_{\varepsilon}(\theta, I, w)$ defined by

$$
H_{\varepsilon}:=\mathcal{H}_{\varepsilon} \circ A=\varepsilon^{-2} \mathcal{H} \circ \varepsilon A
$$

where $A$ is the map defined in (2.50). The associated symplectic form is given in (2.54).

By Lemma 2.9 (see also (2.35), (2.49)), in the variables $(\theta, I, w)$ the quadratic Hamiltonian $\mathcal{H}_{L}$ defined in (2.20) simply reads, up to a constant, as

$$
\mathcal{N}:=\mathcal{H}_{L} \circ A=\vec{\Omega}(\kappa) \cdot I+\frac{1}{2}\left(\boldsymbol{\Omega}_{W} w, w\right)_{L^{2}},
$$

where $\vec{\Omega}(\kappa) \in \mathbb{R}^{v}$ is defined in (1.18) and $\boldsymbol{\Omega}_{W}$ in (2.19). Thus the Hamiltonian $H_{\varepsilon}$ in $(5.2)$ is

$$
H_{\varepsilon}=\mathcal{N}+\varepsilon P \quad \text { with } \quad P:=P_{\varepsilon} \circ A .
$$

We look for an embedded invariant torus

$$
i: \mathbb{T}^{v} \rightarrow \mathbb{R}^{v} \times \mathbb{R}^{v} \times \mathfrak{H}_{\mathbb{S}^{+}, \Sigma}^{<}, \quad \varphi \mapsto i(\varphi):=(\theta(\varphi), I(\varphi), w(\varphi)) .
$$

of the Hamiltonian vector field $X_{H_{\varepsilon}}:=\left(\partial_{I} H_{\varepsilon},-\partial_{\theta} H_{\varepsilon}, \Pi_{\mathbb{S}^{+}, \Sigma}^{<} J \nabla_{w} H_{\varepsilon}\right)$ filled by quasi-periodic solutions with Diophantine frequency vector $\omega \in \mathbb{R}^{v}$ (which satisfies also first and second order Melnikov non-resonance conditions, see (5.14)-(5.17)). 


\subsection{Nash-Moser Theorem of Hypothetical Conjugation}

For $\alpha \in \mathbb{R}^{v}$, we consider the family of modified Hamiltonians

$$
H_{\alpha}:=\mathcal{N}_{\alpha}+\varepsilon P, \quad \mathcal{N}_{\alpha}:=\alpha \cdot I+\frac{1}{2}\left(w, \boldsymbol{\Omega}_{W} w\right)_{L^{2}},
$$

and the nonlinear operator

$$
\begin{aligned}
\mathcal{F}(i, \alpha) & :=\mathcal{F}(\omega, \kappa, \varepsilon ; i, \alpha):=\omega \cdot \partial_{\varphi} i(\varphi)-X_{H_{\alpha}}(i(\varphi)) \\
& =\left(\begin{array}{lc}
\omega \cdot \partial_{\varphi} \theta(\varphi) & -\alpha-\varepsilon \partial_{I} P(i(\varphi)) \\
\omega \cdot \partial_{\varphi} I(\varphi) & +\varepsilon \partial_{\theta} P(i(\varphi)) \\
\omega \cdot \partial_{\varphi} w(\varphi)-\Pi_{\mathbb{S}^{+}, \Sigma}^{\angle} J\left(\boldsymbol{\Omega}_{W} w(\varphi)+\varepsilon \nabla_{w} P(i(\varphi))\right)
\end{array}\right) .
\end{aligned}
$$

If $\mathcal{F}(i, \alpha)=0$, then the embedding $\varphi \mapsto i(\varphi)$ is an invariant torus for the Hamiltonian vector field $X_{H_{\alpha}}$, filled with quasi-periodic solutions with frequency $\omega$.

Each Hamiltonian $H_{\alpha}$ in (5.4) is invariant under the involution $\overrightarrow{\mathcal{S}}$ and the translations $\vec{\tau}_{\zeta}, \varsigma \in \mathbb{R}$, defined in (2.51) and (2.52), respectively, as

$$
H_{\alpha} \circ \overrightarrow{\mathcal{S}}=H_{\alpha}, \quad H_{\alpha} \circ \vec{\tau}_{\varsigma}=H_{\alpha}, \quad \forall \varsigma \in \mathbb{R} .
$$

We look for a reversible traveling torus embedding $\varphi \mapsto i(\varphi)=(\theta(\varphi), I(\varphi), w(\varphi))$; namely one satisfying

$$
\overrightarrow{\mathcal{S}} i(\varphi)=i(-\varphi), \quad \vec{\tau}_{\varsigma} i(\varphi)=i(\varphi-\vec{\jmath} \varsigma), \quad \forall \varsigma \in \mathbb{R} .
$$

Lemma 5.1. The operator $\mathcal{F}(\cdot, \alpha)$ maps a reversible, respectively traveling, wave into an anti-reversible, respectively traveling, wave variation, according to Definition 3.33 .

Proof. It follows directly by (5.5) and (5.6).

The norm of the periodic components of the embedded torus

$$
\Im(\varphi):=i(\varphi)-(\varphi, 0,0):=(\Theta(\varphi), I(\varphi), w(\varphi)), \quad \Theta(\varphi):=\theta(\varphi)-\varphi,
$$

is $\|\Im\|_{s}^{k_{0}, v}:=\|\Theta\|_{H_{\varphi}^{s}}^{k_{0}, v}+\|I\|_{H_{\varphi}^{s}}^{k_{0}, v}+\|w\|_{s}^{k_{0}, v}$, where

$$
k_{0}:=m_{0}+2
$$

and $m_{0} \in \mathbb{N}$ is the index of non-degeneracy provided by Proposition 4.5 , which only depends on the linear unperturbed frequencies. Thus, $k_{0}$ is considered as an absolute constant and we will often omit to write the dependence of the various constants with respect to $k_{0}$. We look for quasi-periodic solutions of frequency $\omega$ belonging to a $\delta$-neighbourhood (independent of $\varepsilon$ )

$$
\Omega:=\left\{\omega \in \mathbb{R}^{v}: \operatorname{dist}\left(\omega, \vec{\Omega}\left[\kappa_{1}, \kappa_{2}\right]\right)<\delta\right\}, \quad \delta>0,
$$

of the curve $\vec{\Omega}\left[\kappa_{1}, \kappa_{2}\right]$ defined by (1.18). 
Theorem 5.2. (Nash-Moser) There exist positive constants $\mathrm{a}_{0}, \varepsilon_{0}, C$ depending on $\mathbb{S}, k_{0}$ and $\tau \geqq 1$ such that, for all $v=\varepsilon^{\mathrm{a}}$, a $\in\left(0, \mathrm{a}_{0}\right)$ and for all $\varepsilon \in\left(0, \varepsilon_{0}\right)$, there exist

\section{1. a $k_{0}$-times differentiable function}

$$
\begin{aligned}
& \alpha_{\infty}: \Omega \times\left[\kappa_{1}, \kappa_{2}\right] \mapsto \mathbb{R}^{v}, \\
& \alpha_{\infty}(\omega, \kappa):=\omega+r_{\varepsilon}(\omega, \kappa) \quad \text { with } \quad\left|r_{\varepsilon}\right|^{k_{0}, v} \leqq C \varepsilon v^{-1}
\end{aligned}
$$

2. a family of embedded reversible traveling tori $i_{\infty}(\varphi)$ (cfr. (5.7)), defined for all $(\omega, \kappa) \in \Omega \times\left[\kappa_{1}, \kappa_{2}\right]$, satisfying

$$
\left\|i_{\infty}(\varphi)-(\varphi, 0,0)\right\|_{s_{0}}^{k_{0}, v} \leqq C \varepsilon v^{-1}
$$

3. a sequence of $k_{0}$-times differentiable functions $\mu_{j}^{\infty}: \mathbb{R}^{v} \times\left[\kappa_{1}, \kappa_{2}\right] \rightarrow \mathbb{R}$, $j \in \mathbb{S}_{0}^{c}=\mathbb{Z} \backslash(\mathbb{S} \cup\{0\})$, of the form

$$
\mu_{j}^{\infty}(\omega, \kappa)=\mathrm{m}_{\frac{3}{2}}^{\infty}(\omega, \kappa) \Omega_{j}(\kappa)+\mathrm{m}_{1}^{\infty}(\omega, \kappa) j+\mathrm{m}_{\frac{1}{2}}^{\infty}(\omega, \kappa)|j|^{\frac{1}{2}}+\mathrm{r}_{j}^{\infty}(\omega, \kappa),
$$

with $\Omega_{j}(\kappa)$ defined in (1.11), satisfying

$$
\left|\mathrm{m}_{\frac{3}{2}}^{\infty}-1\right|^{k_{0}, v},\left|\mathrm{~m}_{1}^{\infty}\right|^{k_{0}, v},\left|\mathrm{~m}_{\frac{1}{2}}^{\infty}\right|^{k_{0}, v} \leqq C \varepsilon, \quad \sup _{j \in \mathbb{S}_{0}^{c}}\left|\mathfrak{r}_{j}^{\infty}\right|^{k_{0}, v} \leqq C \varepsilon v^{-1}
$$

such that, for all $(\omega, \kappa)$ in the Cantor-like set

$$
\begin{gathered}
\mathcal{C}_{\infty}^{v}:=\left\{(\omega, \kappa) \in \Omega \times\left[\kappa_{1}, \kappa_{2}\right]:|\omega \cdot \ell| \geqq 8 v\langle\ell\rangle^{-\tau}, \forall \ell \in \mathbb{Z}^{v} \backslash\{0\}\right. \\
\left|\omega \cdot \ell+\mu_{j}^{\infty}(\omega, \kappa)\right| \geqq 4 v|j|^{\frac{3}{2}}\langle\ell\rangle^{-\tau} \\
\forall \ell \in \mathbb{Z}^{v}, j \in \mathbb{S}_{0}^{c} \text { with } \vec{\jmath} \cdot \ell+j=0 ; \\
\left|\omega \cdot \ell+\mu_{j}^{\infty}(\omega, \kappa)-\mu_{j^{\prime}}^{\infty}(\omega, \kappa)\right| \geqq 4 v\left\langle|j|^{\frac{3}{2}}-\left|j^{\prime}\right|^{\frac{3}{2}}\right\rangle\langle\ell\rangle^{-\tau} \\
\forall \ell \in \mathbb{Z}^{v}, j, j^{\prime} \in \mathbb{S}_{0}^{c},\left(\ell, j, j^{\prime}\right) \neq(0, j, j) \text { with } \vec{j} \cdot \ell+j-j^{\prime}=0, \\
\left|\omega \cdot \ell+\mu_{j}^{\infty}(\omega, \kappa)+\mu_{j^{\prime}}^{\infty}(\omega, \kappa)\right| \geqq 4 v\left(|j|^{\frac{3}{2}}+\left|j^{\prime}\right|^{\frac{3}{2}}\right)\langle\ell\rangle^{-\tau} \\
\left.\forall \ell \in \mathbb{Z}^{v}, j, j^{\prime} \in \mathbb{S}_{0}^{c}, \text { with } \vec{j} \cdot \ell+j+j^{\prime}=0\right\}
\end{gathered}
$$

the function $i_{\infty}(\varphi):=i_{\infty}(\omega, \kappa, \varepsilon ; \varphi)$ is a solution of $\mathcal{F}\left(\omega, \kappa, \varepsilon ; i_{\infty}, \alpha_{\infty}(\omega, \kappa)\right)=$ 0 . As a consequence, the embedded torus $\varphi \mapsto i_{\infty}(\varphi)$ is invariant for the Hamiltonian vector field $X_{H_{\alpha_{\infty}(\omega, \kappa)}}$ as it is filled by quasi-periodic reversible traveling wave solutions with frequency $\omega$.

We remind that the conditions on the indexes in (5.15)-(5.17) (where $\vec{j} \in \mathbb{Z}^{v}$ is the vector in (2.53)) are due to the fact that we look for traveling wave solutions. These restrictions are essential to prove the measure estimates of the next section. 


\subsection{Measure Estimates}

By (5.10), the function $\alpha_{\infty}(\cdot, \kappa)$ from $\Omega$ into its image $\alpha_{\infty}(\Omega, \kappa)$ is invertible and

$$
\begin{aligned}
& \beta=\alpha_{\infty}(\omega, \kappa)=\omega+r_{\varepsilon}(\omega, \kappa) \Leftrightarrow \\
& \omega=\alpha_{\infty}^{-1}(\beta, \kappa)=\beta+\breve{r}_{\varepsilon}(\beta, \kappa), \quad\left|\breve{r}_{\varepsilon}\right|^{k_{0}, v} \leqq C \varepsilon v^{-1} .
\end{aligned}
$$

Then, for any $\beta \in \alpha_{\infty}\left(\mathcal{C}_{\infty}^{v}\right)$, Theorem 5.2 proves the existence of an embedded invariant torus filled by quasi-periodic solutions with Diophantine frequency $\omega=$ $\alpha_{\infty}^{-1}(\beta, \kappa)$ for the Hamiltonian

$$
H_{\beta}=\beta \cdot I+\frac{1}{2}\left(w, \boldsymbol{\Omega}_{W} w\right)_{L^{2}}+\varepsilon P .
$$

Consider the curve of the unperturbed tangential frequency vector $\vec{\Omega}(\kappa)$ in (1.18). In Theorem 5.3 below we prove that for "most" values of $\kappa \in\left[\kappa_{1}, \kappa_{2}\right]$ the vector $\left(\alpha_{\infty}^{-1}(\vec{\Omega}(\kappa), \kappa), \kappa\right)$ is in $\mathcal{C}_{\infty}^{v}$, obtaining an embedded torus for the Hamiltonian $H_{\varepsilon}$ in (5.2), filled by quasi-periodic solutions with Diophantine frequency vector $\omega=\alpha_{\infty}^{-1}(\vec{\Omega}(\kappa), \kappa)$, denoted $\widetilde{\Omega}$ in Theorem 1.5. Thus $\varepsilon A\left(i_{\infty}(\widetilde{\Omega} t)\right)$, where $A$ is defined in (2.50), is a quasi-periodic traveling wave solution of the water waves equations (2.14) written in the Wahlén variables. Finally, going back to the original Zakharov variables via (2.10) we obtain solutions of (1.3). This proves Theorem 1.5 together with the following measure estimate.

Theorem 5.3. (Measure estimates) Let

$$
v=\varepsilon^{\mathrm{a}}, \quad 0<\mathrm{a}<\min \left\{\mathrm{a}_{0}, 1 /\left(1+k_{0}\right)\right\}, \quad \tau>m_{0}(v+4),
$$

where $m_{0}$ is the index of non-degeneracy given in Proposition 4.5 and $k_{0}:=m_{0}+2$. Then, for $\varepsilon \in\left(0, \varepsilon_{0}\right)$ small enough, the measure of the set

$$
\mathcal{G}_{\varepsilon}:=\left\{\kappa \in\left[\kappa_{1}, \kappa_{2}\right]:\left(\alpha_{\infty}^{-1}(\vec{\Omega}(\kappa), \kappa), \kappa\right) \in \mathcal{C}_{\infty}^{v}\right\}
$$

satisfies $\left|\mathcal{G}_{\varepsilon}\right| \rightarrow \kappa_{2}-\kappa_{1}$ as $\varepsilon \rightarrow 0$.

The rest of this section is devoted to prove Theorem 5.3. By (5.18) we have

$$
\vec{\Omega}_{\varepsilon}(\kappa):=\alpha_{\infty}^{-1}(\vec{\Omega}(\kappa), \kappa)=\vec{\Omega}(\kappa)+\vec{r}_{\varepsilon},
$$

where $\vec{r}_{\varepsilon}(\kappa):=\breve{r}_{\varepsilon}(\vec{\Omega}(\kappa), \kappa)$ satisfies

$$
\left|\partial_{\kappa}^{k} \vec{r}_{\varepsilon}(\kappa)\right| \leqq C \varepsilon v^{-(1+k)}, \quad \forall|k| \leqq k_{0}, \text { uniformly on }\left[\kappa_{1}, \kappa_{2}\right] .
$$

We also denote, with a small abuse of notation, for all $j \in \mathbb{S}_{0}^{c}$,

$$
\mu_{j}^{\infty}(\kappa):=\mu_{j}^{\infty}\left(\vec{\Omega}_{\varepsilon}(\kappa), \kappa\right):=\mathrm{m}_{\frac{3}{2}}^{\infty}(\kappa) \Omega_{j}(\kappa)+\mathrm{m}_{1}^{\infty}(\kappa) j+\mathrm{m}_{\frac{1}{2}}^{\infty}(\kappa)|j|^{\frac{1}{2}}+\mathfrak{r}_{j}^{\infty}(\kappa),
$$

where $\mathrm{m}_{\frac{3}{2}}^{\infty}(\kappa):=\mathrm{m}_{\frac{3}{2}}^{\infty}\left(\vec{\Omega}_{\varepsilon}(\kappa), \kappa\right), \mathrm{m}_{1}^{\infty}(\kappa):=\mathrm{m}_{1}^{\infty}\left(\vec{\Omega}_{\varepsilon}(\kappa), \kappa\right), \mathrm{m}_{\frac{1}{2}}^{\infty}(\kappa):=\mathrm{m}_{\frac{1}{2}}^{\infty}\left(\vec{\Omega}_{\varepsilon}(\kappa), \kappa\right)$ and $\mathfrak{r}_{j}^{\infty}(\kappa):=\mathfrak{r}_{j}^{\infty}\left(\stackrel{\vec{\Omega}}{\Omega}_{\varepsilon}(\kappa), \kappa\right)$. 
By (5.13) and (5.22) we have

$$
\begin{aligned}
& \left|\partial_{\kappa}^{k}\left(\mathrm{~m}_{\frac{3}{2}}^{\infty}(\kappa)-1\right)\right|,\left|\partial_{\kappa}^{k} \mathrm{~m}_{1}^{\infty}(\kappa)\right|,\left|\partial_{\kappa}^{k} \mathrm{~m}_{\frac{1}{2}}^{\infty}(\kappa)\right| \leqq C \varepsilon v^{-k}, \\
& \sup _{j \in \mathbb{S}_{0}^{c}}\left|\partial_{\kappa}^{k} \mathfrak{r}_{j}^{\infty}(\kappa)\right| \leqq C \varepsilon v^{-1-k}, \quad \forall 0 \leqq k \leqq k_{0} .
\end{aligned}
$$

Recalling (5.14)-(5.17), the Cantor set in (5.20) becomes

$$
\begin{aligned}
& \mathcal{G}_{\varepsilon}:=\left\{\kappa \in\left[\kappa_{1}, \kappa_{2}\right]:\left|\vec{\Omega}_{\varepsilon}(\kappa) \cdot \ell\right| \geqq 8 v\langle\ell\rangle^{-\tau}, \forall \ell \in \mathbb{Z}^{v} \backslash\{0\} ;\right. \\
& \left|\vec{\Omega}_{\varepsilon}(\kappa) \cdot \ell+\mu_{j}^{\infty}(\kappa)\right| \geqq 4 v|j|^{\frac{3}{2}}\langle\ell\rangle^{-\tau}, \\
& \forall \ell \in \mathbb{Z}^{v}, j \in \mathbb{S}_{0}^{c}, \text { with } \vec{j} \cdot \ell+j=0 ; \\
& \left|\vec{\Omega}_{\varepsilon}(\kappa) \cdot \ell+\mu_{j}^{\infty}(\kappa)-\mu_{j^{\prime}}^{\infty}(\kappa)\right| \geqq 4 v\left\langle|j|^{\frac{3}{2}}-\left|j^{\prime}\right|^{\frac{3}{2}}\right\rangle\langle\ell\rangle^{-\tau}, \\
& \forall \ell \in \mathbb{Z}^{v}, j, j^{\prime} \in \mathbb{S}_{0}^{c},\left(\ell, j, j^{\prime}\right) \neq(0, j, j) \text { with } \vec{j} \cdot \ell+j-j^{\prime}=0 ; \\
& \left|\vec{\Omega}_{\varepsilon}(\kappa) \cdot \ell+\mu_{j}^{\infty}(\kappa)+\mu_{j^{\prime}}^{\infty}(\kappa)\right| \geqq 4 v\left(|j|^{\frac{3}{2}}+\left|j^{\prime}\right| \frac{3}{2}\right)\langle\ell\rangle^{-\tau}, \\
& \left.\forall \ell \in \mathbb{Z}^{v}, j, j^{\prime} \in \mathbb{S}_{0}^{c} \text { with } \vec{j} \cdot \ell+j+j^{\prime}=0\right\} .
\end{aligned}
$$

We estimate the measure of the complementary set

$$
\begin{aligned}
\mathcal{G}_{\varepsilon}^{c} & :=\left[\kappa_{1}, \kappa_{2}\right] \backslash \mathcal{G}_{\varepsilon} \\
& =\left(\bigcup_{\ell \neq 0} R_{\ell}^{(0)}\right) \cup\left(\bigcup_{\substack{\ell \in \mathbb{Z}^{\nu}, j \in \mathbb{S}_{0}^{c} \\
j \cdot \ell+j=0}} R_{\ell, j}^{(I)}\right) \cup\left(\bigcup_{\substack{\left(\ell, j, j \\
j, \neq(0, j, j), j \neq j^{\prime} \\
j \cdot \ell+j-j^{\prime}=0\right.}} R_{\ell, j, j^{\prime}}^{(I I)}\right) \cup\left(\bigcup_{\substack{\ell \in \mathbb{Z}^{\nu}, j, j^{\prime} \in \mathbb{S}_{0}^{c}, j \cdot \ell+j+j^{\prime}=0}} Q_{\ell, j, j^{\prime}}^{(I I)}\right),
\end{aligned}
$$

where the "nearly-resonant sets"

$$
\begin{aligned}
& R_{\ell}^{(0)}:=\left\{\kappa \in\left[\kappa_{1}, \kappa_{2}\right]:\left|\vec{\Omega}_{\varepsilon}(\kappa) \cdot \ell\right|<8 v\langle\ell\rangle^{-\tau}\right\}, \\
& R_{\ell, j}^{(I)}:=\left\{\kappa \in\left[\kappa_{1}, \kappa_{2}\right]:\left|\vec{\Omega}_{\varepsilon}(\kappa) \cdot \ell+\mu_{j}^{\infty}(\kappa)\right|<4 v|j|^{\frac{3}{2}}\langle\ell\rangle^{-\tau}\right\}, \\
& R_{\ell, j, j^{\prime}}^{(I I)}:=\left\{\kappa \in\left[\kappa_{1}, \kappa_{2}\right]:\left|\vec{\Omega}_{\varepsilon}(\kappa) \cdot \ell+\mu_{j}^{\infty}(\kappa)-\mu_{j^{\prime}}^{\infty}(\kappa)\right|<4 v\left\langle|j|^{\frac{3}{2}}-\left|j^{\prime}\right|^{\frac{3}{2}}\right\rangle\langle\ell\rangle^{-\tau}\right\}, \\
& Q_{\ell, j, j^{\prime}}^{(I I)}:=\left\{\kappa \in\left[\kappa_{1}, \kappa_{2}\right]:\left|\vec{\Omega}_{\varepsilon}(\kappa) \cdot \ell+\mu_{j}^{\infty}(\kappa)+\mu_{j^{\prime}}^{\infty}(\kappa)\right|<4 v\left(|j|^{\frac{3}{2}}+\left|j^{\prime}\right|^{\frac{3}{2}}\right)\langle\ell\rangle^{-\tau}\right\} .
\end{aligned}
$$

Note that in the third union in (5.26) we may require $j \neq j^{\prime}$ because $R_{\ell, j, j}^{(I I)} \subset R_{\ell}^{(0)}$. In the sequel we shall always suppose the momentum conditions on the indexes $\ell, j, j^{\prime}$ written in (5.26). Some of the above sets are empty.

Lemma 5.4. Consider the sets in (5.26)-(5.30). For $\varepsilon \in\left(0, \varepsilon_{0}\right)$ small enough, we have that

$$
\text { 1. If } R_{\ell, j}^{(I)} \neq \emptyset \text { then }|j|^{\frac{3}{2}} \leqq C\langle\ell\rangle \text {; }
$$


2. If $R_{\ell, j, j^{\prime}}^{(I I)} \neq \emptyset$ then $\left.|| j\right|^{\frac{3}{2}}-\left|j^{\prime}\right|^{\frac{3}{2}} \mid \leqq C\langle\ell\rangle$;

3. If $Q_{\ell, j, j^{\prime}}^{(I I)} \neq \emptyset$ then $|j|^{\frac{3}{2}}+\left|j^{\prime}\right|^{\frac{3}{2}} \leqq C\langle\ell\rangle$.

Proof. We provide the proof for $R_{\ell, j, j^{\prime}}^{(I I)}$. If $R_{\ell, j, j^{\prime}}^{(I I)} \neq \emptyset$ then there exists $\kappa \in\left[\kappa_{1}, \kappa_{2}\right]$ such that

$$
\left|\mu_{j}^{\infty}(\kappa)-\mu_{j^{\prime}}^{\infty}(\kappa)\right|<\frac{4 v\left\langle|j|^{\frac{3}{2}}-\left|j^{\prime}\right|^{\frac{3}{2}}\right\rangle}{\langle\ell\rangle^{\tau}}+\left|\vec{\Omega}_{\varepsilon}(\kappa) \cdot \ell\right| \leqq\left. 4 v|| j\right|^{\frac{3}{2}}-\left|j^{\prime}\right|^{\frac{3}{2}} \mid+C\langle\ell\rangle .
$$

By (5.23) we have

$$
\begin{aligned}
\mu_{j}^{\infty}(\kappa)-\mu_{j^{\prime}}^{\infty}(\kappa)= & \mathrm{m}_{\frac{3}{2}}^{\infty}(\kappa)\left(\Omega_{j}(\kappa)-\Omega_{j^{\prime}}(\kappa)\right)+\mathrm{m}_{1}^{\infty}(\kappa)\left(j-j^{\prime}\right) \\
& +\mathrm{m}_{\frac{1}{2}}^{\infty}(\kappa)\left(|j|^{\frac{1}{2}}-\left|j^{\prime}\right|^{\frac{1}{2}}\right)+\mathfrak{r}_{j}^{\infty}(\kappa)-\mathfrak{r}_{j^{\prime}}^{\infty}(\kappa) .
\end{aligned}
$$

Then, by (5.24)-(5.25) with $k=0,(4.5)-(4.6)$, the momentum condition $j-j^{\prime}=$ $-\vec{j} \cdot \ell$, and the elementary inequality ||$j^{\frac{3}{2}}-\left.\left|j^{\prime}\right|^{\frac{3}{2}}|\geqq|\left|j^{\frac{1}{2}}-\right| j^{\prime}\right|^{\frac{1}{2}} \mid$, we deduce the lower bound

$$
\begin{aligned}
\left|\mu_{j}^{\infty}(\kappa)-\mu_{j^{\prime}}^{\infty}(\kappa)\right| \geqq & (1-C \varepsilon) \sqrt{\kappa}\left(\left.|| j\right|^{\frac{3}{2}}-\left|j^{\prime}\right|^{\frac{3}{2}} \mid-C\right) \\
& -C \varepsilon|\vec{j} \cdot \ell|-\left.C \varepsilon|| j\right|^{\frac{1}{2}}-\left|j^{\prime}\right|^{\frac{1}{2}} \mid-C \varepsilon v^{-1} \\
\geqq & \left.\frac{\sqrt{\kappa}}{2}|| j\right|^{\frac{3}{2}}-\left|j^{\prime}\right|^{\frac{3}{2}}|-C \varepsilon| \ell \mid-C^{\prime}-C \varepsilon v^{-1} .
\end{aligned}
$$

Combining (5.31) and (5.32), we deduce $\left.|| j\right|^{\frac{3}{2}}-\left|j^{\prime}\right|^{\frac{3}{2}} \mid \leqq C\langle\ell\rangle$, for $\varepsilon$ small enough.

In order to estimate the measure of the sets (5.27)-(5.30) that are nonempty, the key point is to prove that the perturbed frequencies satisfy estimates similar to (4.7)-(4.10).

Lemma 5.5. (Perturbed transversality) For $\varepsilon \in\left(0, \varepsilon_{0}\right)$ small enough and for all $\kappa \in\left[\kappa_{1}, \kappa_{2}\right]$,

$$
\begin{aligned}
& \max _{0 \leqq n \leqq m_{0}}\left|\partial_{\kappa}^{n} \vec{\Omega}_{\varepsilon}(\kappa) \cdot \ell\right| \geqq \frac{\rho_{0}}{2}\langle\ell\rangle, \quad \forall \ell \in \mathbb{Z}^{\nu} \backslash\{0\} ; \\
& \left\{\begin{array}{l}
\max _{0 \leqq n \leqq m_{0}}\left|\partial_{\kappa}^{n}\left(\vec{\Omega}_{\varepsilon}(\kappa) \cdot \ell+\mu_{j}^{\infty}(\kappa)\right)\right| \geqq \frac{\rho_{0}}{2}\langle\ell\rangle \\
\vec{J} \cdot \ell+j=0, \quad \ell \in \mathbb{Z}^{v}, j \in \mathbb{S}_{0}^{c} ;
\end{array}\right. \\
& \left\{\begin{array}{l}
\max _{0 \leqq n \leqq m_{0}}\left|\partial_{\kappa}^{n}\left(\vec{\Omega}_{\varepsilon}(\kappa) \cdot \ell+\mu_{j}^{\infty}(\kappa)-\mu_{j^{\prime}}^{\infty}(\kappa)\right)\right| \geqq \frac{\rho_{0}}{2}\langle\ell\rangle \\
\vec{J} \cdot \ell+j-j^{\prime}=0, \quad \ell \in \mathbb{Z}^{v}, j, j^{\prime} \in \mathbb{S}_{0}^{c},\left(\ell, j, j^{\prime}\right) \neq(0, j, j) ;
\end{array}\right. \\
& \left\{\begin{array}{l}
\max _{0 \leqq n \leqq m_{0}}\left|\partial_{\kappa}^{n}\left(\vec{\Omega}_{\varepsilon}(\kappa) \cdot \ell+\mu_{j}^{\infty}(\kappa)+\mu_{j^{\prime}}^{\infty}(\kappa)\right)\right| \geqq \frac{\rho_{0}}{2}\langle\ell\rangle \\
\vec{j} \cdot \ell+j+j^{\prime}=0, \quad \ell \in \mathbb{Z}^{v}, j, j^{\prime} \in \mathbb{S}_{0}^{c} .
\end{array}\right.
\end{aligned}
$$

We recall that $\rho_{0}$ is the amount of non-degeneracy that has been defined in Proposition 4.5. 
Proof. We prove (5.36). The proofs of (5.33), (5.34) and (5.36) are similar. By (5.23) we have

$$
\begin{aligned}
\vec{\Omega}_{\varepsilon}(\kappa) \cdot \ell & +\mu_{j}^{\infty}(\kappa)-\mu_{j^{\prime}}^{\infty}(\kappa)=\vec{\Omega}(\kappa) \cdot \ell+\vec{r}_{\varepsilon}(\kappa) \cdot \ell+\Omega_{j}(\kappa)-\Omega_{j^{\prime}}(\kappa) \\
& +\left(\mathrm{m}_{\frac{3}{2}}^{\infty}(\kappa)-1\right)\left(\Omega_{j}(\kappa)-\Omega_{j^{\prime}}(\kappa)\right)+\mathrm{m}_{1}^{\infty}(\kappa)\left(j-j^{\prime}\right) \\
& +\mathrm{m}_{\frac{1}{2}}^{\infty}(\kappa)\left(|j|^{\frac{1}{2}}-\left|j^{\prime}\right|^{\frac{1}{2}}\right)+\mathfrak{r}_{j}^{\infty}(\kappa)-\mathfrak{r}_{j^{\prime}}^{\infty}(\kappa) .
\end{aligned}
$$

By Lemma 4.4 we get that, for any $n \in\left\{0, \ldots, m_{0}\right\}$,

$$
\left|\partial_{\kappa}^{n}\left(\Omega_{j}(\kappa)-\Omega_{j^{\prime}}(\kappa)\right)\right| \leqq C(\kappa)|| j^{\frac{3}{2}}-\left|j^{\prime}\right|^{\frac{3}{2}} \mid+C \leqq C^{\prime}(\kappa)\langle\ell\rangle,
$$

because, by Lemma 5.4-2, we can restrict to indexes $\ell, j, j^{\prime}$ such that $\left.|| j\right|^{\frac{3}{2}}-$ $\left|j^{\prime}\right|^{\frac{3}{2}} \mid \leqq C\langle\ell\rangle$. Furthermore

$$
\left.|| j\right|^{\frac{1}{2}}-\left.\left|j^{\prime}\right|^{\frac{1}{2}}|\leqq|\left|j^{\frac{3}{2}}-\right| j^{\prime}\right|^{\frac{3}{2}} \mid \leqq C\langle\ell\rangle
$$

Therefore, by (5.37), (5.24), (5.25), (5.22), (5.38), (5.39), and the momentum condition $j-j^{\prime}=-\vec{j} \cdot \ell$, we have that, for any $n \in\left\{0, \ldots, m_{0}\right\}$,

$$
\begin{aligned}
\left|\partial_{\kappa}^{n}\left(\vec{\Omega}_{\varepsilon}(\kappa) \cdot \ell+\mu_{j}^{\infty}(\kappa)-\mu_{j^{\prime}}^{\infty}(\kappa)\right)\right| \geqq & \left|\partial_{\kappa}^{n}\left(\vec{\Omega}(\kappa) \cdot \ell+\Omega_{j}(\kappa)-\Omega_{j^{\prime}}(\kappa)\right)\right| \\
& -C \varepsilon v^{-\left(1+m_{0}\right)}\langle\ell\rangle .
\end{aligned}
$$

Since $\vec{\Omega}(\kappa) \cdot \ell+\Omega_{j}(\kappa)-\Omega_{j^{\prime}}(\kappa)$ satisfies (4.10), we deduce that

$$
\max _{0 \leqq n \leqq m_{0}}\left|\partial_{\kappa}^{n}\left(\vec{\Omega}_{\varepsilon}(\kappa) \cdot \ell+\mu_{j}^{\infty}(\kappa)-\mu_{j^{\prime}}^{\infty}(\kappa)\right)\right| \geqq \rho_{0}\langle\ell\rangle-C \varepsilon v^{-\left(1+m_{0}\right)}\langle\ell\rangle \geqq \frac{\rho_{0}}{2}\langle\ell\rangle
$$

for $\varepsilon>0$ small enough.

As an application of Rüssmann Theorem 17.1 in [37], we deduce the following result:

Lemma 5.6. (Estimates of the resonant sets) The measure of the sets (5.26)- (5.30) satisfy

$$
\begin{aligned}
& \left|R_{\ell}^{(0)}\right| \lesssim\left(v\langle\ell\rangle^{-(\tau+1)}\right)^{\frac{1}{m_{0}}}, \quad\left|R_{\ell, j}^{(I)}\right| \lesssim\left(v|j|^{\frac{3}{2}}\langle\ell\rangle^{-(\tau+1)}\right)^{\frac{1}{m_{0}}} \\
& \left|R_{\ell, j, j^{\prime}}^{(I I)}\right| \lesssim\left(v\left\langle|j|^{\frac{3}{2}}-\left|j^{\prime}\right|^{\frac{3}{2}}\right\rangle\langle\ell\rangle^{-(\tau+1)}\right)^{\frac{1}{m_{0}}} \\
& \left|Q_{\ell, j, j^{\prime}}^{(I I)}\right| \lesssim\left(v\left(|j|^{\frac{3}{2}}+\left|j^{\prime}\right|^{\frac{3}{2}}\right)\langle\ell\rangle^{-(\tau+1)}\right)^{\frac{1}{m_{0}}}
\end{aligned}
$$

and, recalling Lemma 5.4,

$$
\left|R_{\ell, j}^{(I)}\right|,\left|R_{\ell, j, j^{\prime}}^{(I I)}\right|,\left|Q_{\ell, j, j^{\prime}}^{(I I)}\right| \lesssim\left(v\langle\ell\rangle^{-\tau}\right)^{\frac{1}{m_{0}}} .
$$


Proof. We estimate $R_{\ell, j, j^{\prime}}^{(I I)}$ defined in (5.29). The other cases follow similarly. Defining $f_{\ell, j, j^{\prime}}(\kappa):=\left(\vec{\Omega}_{\varepsilon}(\kappa) \cdot \ell+\mu_{j}^{\infty}(\kappa)-\mu_{j^{\prime}}^{\infty}(\kappa)\right)\langle\ell\rangle^{-1}$, we write

$$
R_{\ell, j, j^{\prime}}^{(I I)}=\left\{\kappa \in\left[\kappa_{1}, \kappa_{2}\right]:\left|f_{\ell, j, j^{\prime}}(\kappa)\right|<4 v\left\langle|j|^{\frac{3}{2}}-\left|j^{\prime}\right|^{\frac{3}{2}}\right\rangle\langle\ell\rangle^{-\tau-1}\right\} .
$$

By Lemma 5.4-2 we restrict to indexes satisfying $\left.|| j\right|^{\frac{3}{2}}-\left|j^{\prime}\right|^{\frac{3}{2}} \mid \leqq C\langle\ell\rangle$. By (5.36),

$$
\max _{0 \leqq n \leqq m_{0}}\left|\partial_{\kappa}^{n} f_{\ell, j, j^{\prime}}(\kappa)\right| \geqq \rho_{0} / 2, \quad \forall \kappa \in\left[\kappa_{1}, \kappa_{2}\right]
$$

In addition, by (5.21)-(5.25), Lemma 4.4, the momentum condition $\left|j-j^{\prime}\right|=|\vec{J} \cdot \ell|$, and (5.39), we deduce that $\max _{0 \leqq n \leqq k_{0}}\left|\partial_{\kappa}^{n} f_{\ell, j, j^{\prime}}(\kappa)\right| \leqq C$ for all $\kappa \in\left[\kappa_{1}, \kappa_{2}\right]$, provided $\varepsilon v^{-\left(1+k_{0}\right)}$ is small enough, namely, by (5.19) and $\varepsilon$ small enough. In particular, $f_{\ell, j, j^{\prime}}$ is of class $\mathcal{C}^{k_{0}-1}=\mathcal{C}^{m_{0}+1}$. Thus Theorem 17.1 in [37] applies.

Proof of Theorem 5.3 completed. We estimate the measure of all the sets in (5.26). By Lemma 5.4 and Lemma 5.6 we have that

$$
\begin{aligned}
& \left|\bigcup_{\ell \neq 0} R_{\ell}^{(0)}\right| \leqq \sum_{\ell \neq 0}\left|R_{\ell}^{(0)}\right| \lesssim \sum_{\ell \neq 0}\left(\frac{v}{\langle\ell\rangle^{\tau+1}}\right)^{\frac{1}{m_{0}}}, \\
& \left|\bigcup_{\substack{\ell, j \in \mathbb{S}_{0}^{C} \\
j \cdot \ell+j=0}} R_{\ell, j}^{(I)}\right| \leqq \sum_{\substack{|j| \leq C\langle\ell\rangle^{\frac{2}{3}} \\
j \cdot \ell+j=0}}\left|R_{\ell, j}^{(I)}\right| \lesssim \sum_{|j| \leqq C\langle\ell\rangle^{\frac{2}{3}}}\left(\frac{v}{\langle\ell\rangle^{\tau}}\right)^{\frac{1}{m_{0}}} \lesssim \sum_{\ell \in \mathbb{Z}^{v}} \frac{v^{\frac{1}{m_{0}}}}{\langle\ell\rangle^{\frac{\tau}{m_{0}}-\frac{2}{3}}},
\end{aligned}
$$

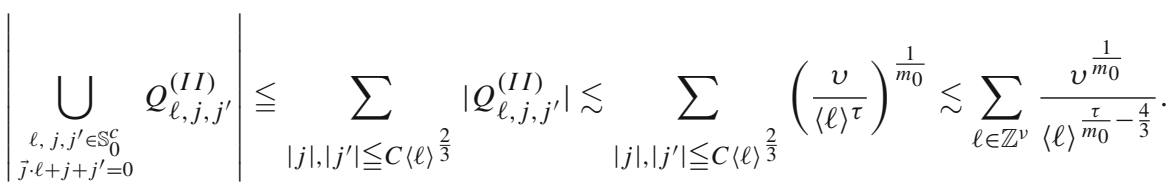

We are left with estimating the measure of

$$
\bigcup_{\substack{\left(\ell, j, j^{\prime}\right) \neq(0, j, j), j \neq j^{\prime} \\ j \cdot \ell+j-j^{\prime}=0}} R_{\ell, j, j^{\prime}}^{(I I)}=\left(\bigcup_{\substack{\ell, j \in \mathbb{S}_{0}^{c} \\ j \cdot \ell+2 j=0}} R_{\ell, j,-j}^{(I I)}\right) \cup\left(\bigcup_{\substack{\ell, j, j^{\prime},|j| \neq\left|j^{\prime}\right| \\ j \cdot \ell+j-j^{\prime}=0}} R_{\ell, j, j^{\prime}}^{(I I)}\right) .
$$

By the momentum condition $\vec{j} \cdot \ell+2 j=0$ we get $|j| \leqq C\langle\ell\rangle$, and, by Lemma 5.6,

$$
\left|\bigcup_{\ell, j \in \mathbb{S}_{0}^{c}, \vec{j} \cdot \ell+2 j=0} R_{\ell, j,-j}^{(I I)}\right| \leqq \sum_{|j| \leqq C\langle\ell\rangle}\left|R_{\ell, j,-j}^{(I I)}\right| \lesssim \sum_{|j| \leqq C\langle\ell\rangle}\left(\frac{v}{\langle\ell\rangle^{\tau}}\right)^{\frac{1}{m_{0}}} \lesssim \sum_{\ell \in \mathbb{Z}^{\nu}} \frac{v^{\frac{1}{m_{0}}}}{\langle\ell\rangle^{\frac{\tau}{m_{0}}}-1} .
$$


Finally we estimate the measure of the second union in (5.43). By Lemma 5.4-2 we can restrict to indexes satisfying $\left.|| j\right|^{3 / 2}-\left|j^{\prime}\right|^{3 / 2} \mid \leqq C\langle\ell\rangle$. Now, for any $|j| \neq\left|j^{\prime}\right|$, we have

$$
\begin{aligned}
\left.|| j\right|^{\frac{3}{2}}-\left|j^{\prime}\right|^{\frac{3}{2}} \mid & =\left.|| j\right|^{\frac{1}{2}}-\left|j^{\prime}\right|^{\frac{1}{2}} \mid\left(|j|+\left|j^{\prime}\right|+|j|^{\frac{1}{2}}\left|j^{\prime}\right|^{\frac{1}{2}}\right) \\
& \geqq \frac{|j|+\left|j^{\prime}\right|+|j|^{\frac{1}{2}}\left|j^{\prime}\right|^{\frac{1}{2}}}{|j|^{\frac{1}{2}}+\left|j^{\prime}\right|^{\frac{1}{2}}} \geqq \frac{|j|^{\frac{1}{2}}+\left|j^{\prime}\right|^{\frac{1}{2}}}{2},
\end{aligned}
$$

implying the upper bounds $|j|,\left|j^{\prime}\right| \leqq C\langle\ell\rangle^{2}$. Hence

$\left|\bigcup_{\substack{\ell, j, j^{\prime},|j| \neq\left|j^{\prime}\right| \\ j, \ell+j-j^{\prime}=0}} R_{\ell, j, j^{\prime}}^{(I I)}\right| \leqq \sum_{\substack{|j|,\left|j^{\prime}\right| \leqq C\langle\ell\rangle^{2} \\ \mid R^{\prime}}}^{(I I)} \mid \lesssim \sum_{\ell, j, j^{\prime}}\left(\frac{v}{\langle\ell\rangle^{\tau}}\right)^{\frac{1}{m_{0}}} \lesssim \sum_{\ell \in \mathbb{Z}^{v}} \frac{v^{\frac{1}{m_{0}}}}{\langle\ell\rangle^{\frac{\tau}{m_{0}}-4}}$.

As $\frac{\tau}{m_{0}}-4>v$ by (5.19), all the series in (5.40), (5.41), (5.42), (5.44), (5.45) are convergent, and we deduce

$$
\left|\mathcal{G}_{\varepsilon}^{c}\right| \leqq C v^{\frac{1}{m_{0}}}
$$

For $v=\varepsilon^{\mathrm{a}}$ as in (5.19), we get $\left|\mathcal{G}_{\varepsilon}\right| \geqq \kappa_{2}-\kappa_{1}-C \varepsilon^{\mathrm{a} / m_{0}}$. The proof of Theorem 5.3 is concluded.

\section{Approximate Inverse}

In order to implement a convergent Nash-Moser scheme that leads to a solution of $\mathcal{F}(i, \alpha)=0$, where $\mathcal{F}(i, \alpha)$ is the nonlinear operator defined in (5.5), we construct an almost approximate right inverse of the linearized operator

$$
\mathrm{d}_{i, \alpha} \mathcal{F}\left(i_{0}, \alpha_{0}\right)[\widehat{\imath}, \widehat{\alpha}]=\omega \cdot \partial_{\varphi} \widehat{\imath}-\mathrm{d}_{i} X_{H_{\alpha}}\left(i_{0}(\varphi)\right)[\widehat{\imath}]-(\widehat{\alpha}, 0,0) .
$$

Note that $\mathrm{d}_{i, \alpha} \mathcal{F}\left(i_{0}, \alpha_{0}\right)=\mathrm{d}_{i, \alpha} \mathcal{F}\left(i_{0}\right)$ is independent of $\alpha_{0}$. We assume that the torus $i_{0}(\varphi)=\left(\theta_{0}(\varphi), I_{0}(\varphi), w_{0}(\varphi)\right)$ is reversible and traveling, according to (5.7).

In the sequel we shall assume the smallness condition, for some $\mathrm{k}:=\mathrm{k}(\tau, v)>$ 0 ,

$$
\varepsilon v^{-\mathrm{k}} \ll 1
$$

We closely follow the strategy presented in [4] and implemented for the water waves equations in $[2,6]$. The main novelty is to check that this construction preserves the momentum preserving properties needed for the search of traveling waves. Therefore, along this section we shall focus on this verification. The estimates are very similar to those in $[2,6]$.

First of all, we state tame estimates for the composition operator induced by the Hamiltonian vector field $X_{P}=\left(\partial_{I} P,-\partial_{\theta} P, \Pi_{\mathbb{S}^{+}, \Sigma}^{L} J \nabla_{w} P\right)$ in (5.5). 
Lemma 6.1. (Estimates of the perturbation $P$ ) Let $\Im(\varphi)$ in (5.8) satisfy $\|\Im\|_{3 s_{0}+2 k_{0}+5}^{k_{0}, v}$ $\leqq 1$. Then, for any $s \geqq s_{0},\left\|X_{P}(i)\right\|_{s}^{k_{0}, v} \lesssim s 1+\|\Im\|_{s+2 s_{0}+2 k_{0}+3}^{k_{0}, v}$, and, for all $\widehat{\imath}:=(\widehat{\theta}, \widehat{I}, \widehat{w})$,

$$
\begin{gathered}
\left\|\mathrm{d}_{i} X_{P}(i)[\widehat{\imath}]\right\|_{s}^{k_{0}, v} \lesssim s\|\widehat{\imath}\|_{s+1}^{k_{0}, v}+\|\mathfrak{I}\|_{s+2 s_{0}+2 k_{0}+4}^{k_{0}, v}\|\widehat{\imath}\|_{s_{0}+1}^{k_{0}, v}, \\
\left\|\mathrm{~d}_{i}^{2} X_{P}(i)[\widehat{\imath}, \widehat{\imath}]\right\|_{s}^{k_{0}, v} \lesssim s\|\widehat{\imath}\|_{s+1}^{k_{0}, v}\|\widehat{\imath}\|_{s_{0}+1}^{k_{0}, v}+\|\mathfrak{I}\|_{s+2 s_{0}+2 k_{0}+5}^{k_{0}, v}\left(\|\widehat{\imath}\|_{s_{0}+1}^{k_{0}, v}\right)^{2} .
\end{gathered}
$$

Proof. The proof goes as in Lemma 5.1 of [6], using also the estimates of the Dirichlet-Neumann operator in Lemma 3.10.

Throughout this section, we assume the following hypothesis, which is verified by the approximate solutions obtained at each step of the Nash-Moser Theorem 9.2:

- ANSATZ. The map $(\omega, \kappa) \mapsto \mathfrak{I}_{0}(\omega, \kappa)=i_{0}(\varphi ; \omega, \kappa)-(\varphi, 0,0)$ is $k_{0}$-times differentiable with respect to the parameters $(\omega, \kappa) \in \mathbb{R}^{v} \times\left[\kappa_{1}, \kappa_{2}\right]$ and, for some $\mu:=\mu(\tau, v)>0, v \in(0,1)$,

$$
\left\|\Im_{0}\right\|_{s_{0}+\mu}^{k_{0}, v}+\left|\alpha_{0}-\omega\right|^{k_{0}, v} \leqq C \varepsilon v^{-1}
$$

As in $[2,4,6]$, we first modify the approximate torus $i_{0}(\varphi)$ to obtain a nearby isotropic torus $i_{\delta}(\varphi)$, namely such that the pull-back 1 -form $i_{\delta}^{*} \Lambda$ is closed, where $\Lambda$ is the Liouville 1-form defined in (2.55). We first consider the pull-back 1-form

$$
\begin{aligned}
& i_{0}^{*} \Lambda=\sum_{k=1}^{v} a_{k}(\varphi) \mathrm{d} \varphi_{k}, \\
& a_{k}(\varphi):=-\left(\left[\partial_{\varphi} \theta_{0}(\varphi)\right]^{\top} I_{0}(\varphi)\right)_{k}+\frac{1}{2}\left(J_{\angle}^{-1} w_{0}(\varphi), \partial_{\varphi_{k}} w_{0}(\varphi)\right)_{L^{2}},
\end{aligned}
$$

and its exterior differential

$$
i_{0}^{*} \mathcal{W}=\mathrm{d} i_{0}^{*} \Lambda=\sum_{1 \leqq k<j \leqq v} A_{k j} \mathrm{~d} \varphi_{k} \wedge \mathrm{d} \varphi_{j}, \quad A_{k j}(\varphi):=\partial_{\varphi_{k}} a_{j}(\varphi)-\partial_{\varphi_{j}} a_{k}(\varphi)
$$

By the formula given in Lemma 5 in [4], we deduce, if $\omega$ belongs to $\mathrm{DC}(v, \tau)$, the estimate

$$
\left\|A_{k j}\right\|_{s}^{k_{0}, v} \lesssim_{s} v^{-1}\left(\|Z\|_{s+\tau\left(k_{0}+1\right)+k_{0}+1}^{k_{0}, v}+\|Z\|_{s_{0}+1}^{k_{0}, v}\left\|\Im_{0}\right\|_{s+\tau\left(k_{0}+1\right)+k_{0}+1}^{k_{0}, v}\right),
$$

where $Z(\varphi)$ is the "error function"

$$
Z(\varphi):=\mathcal{F}\left(i_{0}, \alpha_{0}\right)(\varphi)=\omega \cdot \partial_{\varphi} i_{0}(\varphi)-X_{H_{\alpha_{0}}}\left(i_{0}(\varphi)\right)
$$

Note that if $Z(\varphi)=0$, the torus $i_{0}(\varphi)$ is invariant for $X_{H_{\alpha_{0}}}$ and the 1 -form $i_{0}^{*} \Lambda$ is closed, namely the torus $i_{0}(\varphi)$ is isotropic. We denote below the Laplacian $\Delta_{\varphi}:=$ $\sum_{k=1}^{v} \partial_{\varphi_{k}}^{2}$. 
Lemma 6.2. (Isotropic torus) The torus $i_{\delta}(\varphi):=\left(\theta_{0}(\varphi), I_{\delta}(\varphi), w_{0}(\varphi)\right)$, defined by

$$
\begin{aligned}
I_{\delta}(\varphi) & :=I_{0}(\varphi)+\left[\partial_{\varphi} \theta_{0}(\varphi)\right]^{-\top} \rho(\varphi), \\
\rho & =\left(\rho_{j}\right)_{j=1, \ldots, \nu}, \quad \rho_{j}(\varphi):=\Delta_{\varphi}^{-1} \sum_{k=1}^{v} \partial_{\varphi_{k}} A_{k j}(\varphi),
\end{aligned}
$$

is isotropic. Moreover, there is $\sigma:=\sigma(\nu, \tau)$ such that, for all $s \geqq s_{0}$,

$$
\begin{aligned}
\left\|I_{\delta}-I_{0}\right\|_{s}^{k_{0}, v} & \lesssim_{s}\left\|\Im_{0}\right\|_{s+1}^{k_{0}, v}, \\
\left\|I_{\delta}-I_{0}\right\|_{s}^{k_{0}, v} & \lesssim_{s} v^{-1}\left(\|Z\|_{s+\sigma}^{k_{0}, v}+\|Z\|_{s_{0}+\sigma}^{k_{0}, v}\left\|\Im_{0}\right\|_{s+\sigma}^{k_{0}, v}\right) \\
\left\|\mathcal{F}\left(i_{\delta}, \alpha_{0}\right)\right\|_{s}^{k_{0}, v} & \lesssim_{s}\|Z\|_{s+\sigma}^{k_{0}, v}+\|Z\|_{s_{0}+\sigma}^{k_{0}, v}\left\|\Im_{0}\right\|_{s+\sigma}^{k_{0}, v} \\
\left\|\mathrm{~d}_{i}\left(i_{\delta}\right)[\widehat{\imath}]\right\|_{s_{1}} & \lesssim_{s_{1}}\|\widehat{\imath}\|_{s_{1}+1},
\end{aligned}
$$

for $s_{1} \leqq s_{0}+\mu(c f r .(6.1))$. Furthermore $i_{\delta}(\varphi)$ is a reversible and traveling torus, cfr. (5.7).

Proof. Since $i_{0}(\varphi)$ is a traveling torus (see (3.52)), in order to prove that $i_{\delta}(\varphi)$ is a traveling torus it is sufficient to prove that $I_{\delta}(\varphi-\vec{j} \varsigma)=I_{\delta}(\varphi)$, for any $\varsigma \in \mathbb{R}$. In view of (6.3), this follows by checking that $\partial_{\varphi} \theta_{0}(\varphi-\vec{\jmath} \varsigma)=\partial_{\varphi} \theta_{0}(\varphi)$ and $\rho(\varphi-\vec{\jmath} \varsigma)=\rho(\varphi)$ for any $\varsigma \in \mathbb{R}$. The first identity is a trivial consequence of the fact that $\theta_{0}(\varphi-\vec{\jmath} \varsigma)=\theta_{0}(\varphi)-\vec{\jmath} \varsigma$ for any $\varsigma \in \mathbb{R}$, whereas the second one follows once we prove that the functions $a_{k}(\varphi)$ defined in (6.2) satisfy

$$
a_{k}(\varphi-\vec{\jmath} \varsigma)=a_{k}(\varphi) \quad \forall \varsigma \in \mathbb{R}, \quad \forall k=1, \ldots, \nu .
$$

Using that $i_{0}(\varphi)$ is a traveling torus, we get, for any $\varsigma \in \mathbb{R}$,

$$
\begin{aligned}
\left(\partial_{\varphi_{k}} w_{0}(\varphi-\vec{\jmath} \varsigma), J_{L}^{-1} w_{0}(\varphi-\vec{\jmath} \varsigma)\right)_{L^{2}} & =\left(\partial_{\varphi_{k}} \tau_{\varsigma} w_{0}(\varphi), J_{\angle}^{-1} \tau_{\varsigma} w_{0}(\varphi)\right)_{L^{2}} \\
& =\left(\partial_{\varphi_{k}} w_{0}(\varphi), J_{L}^{-1} w_{0}(\varphi)\right)_{L^{2}}
\end{aligned}
$$

and, recalling (6.2), we deduce (6.8). Moreover, since $i_{0}(\varphi)$ is reversible, in order to prove that $i_{\delta}(\varphi)$ is reversible as well, it is sufficient to show that $I_{\delta}(\varphi)$ is even. This follows by (6.2), Lemma 3.23 and $\mathcal{S} J^{-1}=-J^{-1} \mathcal{S}$. Finally, the estimates (6.4)-(6.7) follow for example as in Lemma 5.3 in [2].

In the sequel we denote by $\sigma=\sigma(\nu, \tau)$ constants, which may increase from lemma to lemma, which represent "loss of derivatives".

In order to find an approximate inverse of the linearized operator $\mathrm{d}_{i, \alpha} \mathcal{F}\left(i_{\delta}\right)$, we introduce the symplectic diffeomorphism $G_{\delta}:(\phi, y, \mathrm{w}) \rightarrow(\theta, I, w)$ of the phase space $\mathbb{T}^{v} \times \mathbb{R}^{v} \times \mathfrak{H}_{\mathbb{S}^{+}, \Sigma}^{L}$,

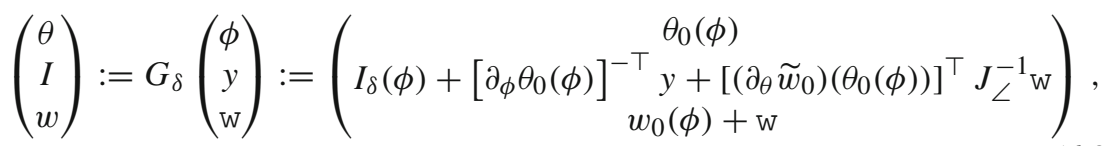

where $\widetilde{w}_{0}(\theta):=w_{0}\left(\theta_{0}^{-1}(\theta)\right)$. It is proved in Lemma 2 of [4] that $G_{\delta}$ is symplectic, because the torus $i_{\delta}$ is isotropic (Lemma 6.2). In the new coordinates, $i_{\delta}$ is the trivial embedded torus $(\phi, y, \mathrm{w})=(\phi, 0,0)$. 
Lemma 6.3. The diffeomorphism $G_{\delta}$ in (6.9) is reversibility and momentum preserving, in the sense that

$$
\overrightarrow{\mathcal{S}} \circ G_{\delta}=G_{\delta} \circ \overrightarrow{\mathcal{S}}, \quad \vec{\tau}_{\varsigma} \circ G_{\delta}=G_{\delta} \circ \vec{\tau}_{\varsigma}, \quad \forall \varsigma \in \mathbb{R},
$$

where $\overrightarrow{\mathcal{S}}$ and $\vec{\tau}_{5}$ are defined respectively in (2.51), (2.52).

Proof. We prove the second identity in (6.10), which, in view of (6.9), (2.52) amounts to

$$
\begin{aligned}
& \theta_{0}(\phi)-\vec{\jmath} \varsigma=\theta_{0}(\phi-\vec{\jmath} \varsigma), \forall \varsigma \in \mathbb{R}, \\
& I_{\delta}(\phi)+\left[\partial_{\phi} \theta_{0}(\phi)\right]^{-\top} y+\left[\left(\partial_{\theta} \widetilde{w}_{0}\right)\left(\theta_{0}(\phi)\right)\right]^{\top} J_{\angle}^{-1} \mathrm{~W} \\
& =I_{\delta}(\phi-\vec{\jmath} \varsigma)+\left[\partial_{\phi} \theta_{0}(\phi-\vec{\jmath} \varsigma)\right]^{-\top} y+\left[\left(\partial_{\theta} \widetilde{w}_{0}\right)\left(\theta_{0}(\phi-\vec{\jmath} \varsigma)\right)\right]^{\top} J_{\angle}^{-1} \tau_{\varsigma} \mathrm{w}, \\
& \tau_{\varsigma} w_{0}(\phi)+\tau_{\varsigma} \mathrm{W}=w_{0}(\phi-\vec{\jmath} \varsigma)+\tau_{\varsigma} \mathrm{W} \text {. }
\end{aligned}
$$

Identities (6.11) and (6.13) follow because $i_{\delta}(\varphi)$ is a traveling torus (Lemma 6.2). For the same reason $I_{\delta}(\phi)=I_{\delta}(\phi-\vec{\jmath} \varsigma)$ and $\partial_{\phi} \theta_{0}(\phi)=\partial_{\phi} \theta_{0}(\phi-\vec{j} \varsigma)$ for any $\varsigma \in \mathbb{R}$. Hence, for verifying (6.12) it is sufficient to check that $\left[\left(\partial_{\theta} \widetilde{w}_{0}\right)\left(\theta_{0}(\phi)\right)\right]^{\top}=$ $\left[\left(\partial_{\theta} \widetilde{w}_{0}\right)\left(\theta_{0}(\phi-\vec{\jmath} \varsigma)\right)\right]^{\top} \tau_{\zeta}$ (we have used that $J_{L}^{-1}$ and $\tau_{\zeta}$ commute by Lemma 3.31), which in turn follows by

$$
\tau_{\varsigma} \circ\left(\partial_{\theta} \widetilde{w}_{0}\right)\left(\theta_{0}(\phi)\right)=\left(\partial_{\theta} \widetilde{w}_{0}\right)\left(\theta_{0}(\phi-\vec{\jmath} \varsigma)\right), \quad \forall \varsigma \in \mathbb{R},
$$

by taking the transpose and using that $\tau_{\varsigma}^{\top}=\tau_{-\varsigma}=\tau_{\varsigma}^{-1}$. We claim that (6.14) is implied by $\widetilde{w}_{0}$ being a traveling wave, that is

$$
\tau_{\varsigma} \widetilde{w}_{0}(\theta, \cdot)=\widetilde{w}_{0}(\theta-\vec{\jmath} \varsigma), \quad \forall \varsigma \in \mathbb{R} .
$$

Indeed, taking the differential of (6.15) with respect to $\theta$, evaluating at $\theta=\theta_{0}(\varphi)$, and using that $\theta_{0}(\varphi)-\vec{\jmath} \varsigma=\theta_{0}(\varphi-\vec{\jmath} \varsigma)$ one deduces (6.14). It remains to prove (6.15). By the definition of $\widetilde{w}_{0}$, and since $w_{0}$ is a traveling wave, we have

$$
\widetilde{w}_{0}(\theta-\vec{\jmath} \varsigma)=w_{0}\left(\theta_{0}^{-1}(\theta-\vec{\jmath} \varsigma)\right)=w_{0}\left(\theta_{0}^{-1}(\theta)-\vec{\jmath} \varsigma\right)=\tau_{\zeta} w_{0}\left(\theta_{0}^{-1}(\theta)\right)=\tau_{\varsigma} \widetilde{w}_{0},
$$

using also that $\theta_{0}^{-1}(\theta-\vec{\jmath} \varsigma)=\theta_{0}^{-1}(\theta)-\vec{\jmath} \varsigma$, which follows by inverting (6.11). The proof of the first identity in (6.10) follows by (6.9), (2.51), the fact that $i_{\delta}$ is reversible, Lemma 3.23 and since $J^{-1}$ and $\mathcal{S}$ anti-commute.

Under the symplectic diffeomorphism $G_{\delta}$, the Hamiltonian vector field $X_{H_{\alpha}}$ changes into

$$
X_{K_{\alpha}}=\left(D G_{\delta}\right)^{-1} X_{H_{\alpha}} \circ G_{\delta} \quad \text { where } \quad K_{\alpha}:=H_{\alpha} \circ G_{\delta} .
$$

By (6.10) and (5.6) we deduce that $K_{\alpha}$ is reversible and momentum preserving, in the sense that

$$
K_{\alpha} \circ \overrightarrow{\mathcal{S}}=K_{\alpha}, \quad K_{\alpha} \circ \vec{\tau}_{\varsigma}=K_{\alpha}, \quad \forall \varsigma \in \mathbb{R} .
$$

The Taylor expansion of $K_{\alpha}$ at the trivial torus $(\phi, 0,0)$ is

$$
K_{\alpha}(\phi, y, \mathrm{w})=K_{00}(\phi, \alpha)+K_{10}(\phi, \alpha) \cdot y+\left(K_{01}(\phi, \alpha), \mathrm{w}\right)_{L^{2}}+\frac{1}{2} K_{20}(\phi) y \cdot y
$$




$$
+\left(K_{11}(\phi) y, \mathrm{w}\right)_{L^{2}}+\frac{1}{2}\left(K_{02}(\phi) \mathrm{w}, \mathrm{w}\right)_{L^{2}}+K_{\geqq 3}(\phi, y, \mathrm{w}),
$$

where $K_{\geq 3}$ collects all terms at least cubic in the variables ( $y$, w). By (5.4) and (6.9), the only Taylor coefficients that depend on $\alpha$ are $K_{00} \in \mathbb{R}, K_{10} \in \mathbb{R}^{v}$ and $K_{01} \in \mathfrak{H}_{\mathbb{S}^{+}, \Sigma}^{<}$, whereas the $v \times v$ symmetric matrix $K_{20}, K_{11} \in \mathcal{L}\left(\mathbb{R}^{\nu}, \mathfrak{H}_{\mathbb{S}^{+}, \Sigma}^{<}\right)$and the linear self-adjoint operator $K_{02}$, acting on $\mathfrak{H}_{\mathbb{S}^{+}, \Sigma}^{<}$, are independent of it.

Differentiating the identities in (6.17) at $(\phi, 0,0)$, we have (recalling (2.51))

$$
\begin{aligned}
& K_{00}(-\phi)=K_{00}(\phi), \quad K_{10}(-\phi)=K_{10}(\phi), \quad K_{20}(-\phi)=K_{20}(\phi), \\
& \mathcal{S} \circ K_{01}(-\phi)=K_{01}(\phi), \quad \mathcal{S} \circ K_{11}(-\phi)=K_{11}(\phi), \\
& K_{02}(-\phi) \circ \mathcal{S}=\mathcal{S} \circ K_{02}(\phi),
\end{aligned}
$$

and, recalling (2.52) and using that $\tau_{\varsigma}^{\top}=\tau_{-\varsigma}=\tau_{\varsigma}^{-1}$, for any $\varsigma \in \mathbb{R}$,

$$
\begin{aligned}
& K_{00}(\phi-\vec{\jmath} \varsigma)=K_{00}(\phi), \quad K_{10}(\phi-\vec{\jmath} \varsigma)=K_{10}(\phi), \quad K_{20}(\phi-\vec{\jmath} \varsigma)=K_{20}(\phi), \\
& K_{01}(\phi-\vec{\jmath} \varsigma)=\tau_{\varsigma} K_{01}(\phi), \quad K_{11}(\phi-\vec{\jmath} \varsigma)=\tau_{\varsigma} K_{11}(\phi), \\
& K_{02}(\phi-\vec{\jmath} \varsigma) \circ \tau_{\varsigma}=\tau_{\varsigma} \circ K_{02}(\phi) .
\end{aligned}
$$

The Hamilton equations associated to (6.18) are

$$
\left\{\begin{array}{l}
\dot{\phi}=K_{10}(\phi, \alpha)+K_{20}(\phi) y+\left[K_{11}(\phi)\right]^{\top} \mathrm{w}+\partial_{y} K_{\geqq 3}(\phi, y, \mathrm{w}) \\
\dot{y}=-\partial_{\phi} K_{00}(\phi, \alpha)-\left[\partial_{\phi} K_{10}(\phi, \alpha)\right]^{\top} y-\left[\partial_{\phi} K_{01}(\phi, \alpha)\right]^{\top} \mathrm{w} \\
-\partial_{\phi}\left(\frac{1}{2} K_{20}(\phi) y \cdot y+\left(K_{11}(\phi) y, \mathrm{w}\right)_{L^{2}}+\frac{1}{2}\left(K_{02}(\phi) \mathrm{w}, \mathrm{w}\right)_{L^{2}}+K_{\geqq 3}(\phi, y, \mathrm{w})\right) \\
\dot{\mathrm{w}}=J_{\angle}\left(K_{01}(\phi, \alpha)+K_{11}(\phi) y+K_{02}(\phi) \mathrm{w}+\nabla_{\mathrm{w}} K_{\geqq 3}(\phi, y, \mathrm{w})\right)
\end{array}\right.
$$

where $\partial_{\phi} K_{10}^{\top}$ is the $v \times v$ transposed matrix and $\partial_{\phi} K_{01}^{\top}, K_{11}^{\top}: \mathfrak{H}_{\mathbb{S}^{+}, \Sigma}^{L} \rightarrow \mathbb{R}^{v}$ are defined by the duality relation $\left(\partial_{\phi} K_{01}[\widehat{\phi}], \mathrm{w}\right)_{L^{2}}=\widehat{\phi} \cdot\left[\partial_{\phi} K_{01}\right]^{\top} \mathrm{w}$ for any $\widehat{\phi} \in \mathbb{R}^{v}$, $\mathrm{w} \in \mathfrak{H}_{\mathbb{S}^{+}, \Sigma}^{<}$. The transpose $K_{11}^{\top}(\phi)$ is defined similarly.

On an exact solution (that is $Z=0$ ), the terms $K_{00}, K_{01}$ in the Taylor expansion (6.18) vanish and $K_{10}=\omega$. More precisely, arguing as in Lemma 5.4 in [2], we have

Lemma 6.4. There is $\sigma:=\sigma(\nu, \tau)>0$, such that, for all $s \geqq s_{0}$,

$$
\begin{aligned}
& \left\|\partial_{\phi} K_{00}\left(\cdot, \alpha_{0}\right)\right\|_{s}^{k_{0}, v}+\left\|K_{10}\left(\cdot, \alpha_{0}\right)-\omega\right\|_{s}^{k_{0}, v}+\left\|K_{01}\left(\cdot, \alpha_{0}\right)\right\|_{s}^{k_{0}, v} \\
& \quad \lesssim_{s}\|Z\|_{s+\sigma}^{k_{0}, v}+\|Z\|_{s_{0}+\sigma}^{k_{0}, v}\left\|\Im_{0}\right\|_{s+\sigma}^{k_{0}, v}, \\
& \left\|\partial_{\alpha} K_{00}\right\|_{s}^{k_{0}, v}+\left\|\partial_{\alpha} K_{10}-\mathrm{Id}\right\|_{s}^{k_{0}, v}+\left\|\partial_{\alpha} K_{01}\right\|_{s}^{k_{0}, v} \lesssim_{s}\left\|\Im_{0}\right\|_{s+\sigma}^{k_{0}, v}, \\
& \left\|K_{20}\right\|_{s}^{k_{0}, v} \lesssim_{s} \varepsilon\left(1+\left\|\Im_{0}\right\|_{s, \sigma}^{k_{0}, v}\right), \\
& \left\|K_{11} y\right\|_{s}^{k_{0}, v} \lesssim_{s} \varepsilon\left(\|y\|_{s}^{k_{0}, v}+\|y\|_{s_{0}}^{k_{0}, v}\left\|\Im_{0}\right\|_{s+\sigma}^{k_{0}, v}\right), \\
& \left\|K_{11}^{\top} \mathrm{w}\right\|_{s}^{k_{0}, v} \lesssim_{s} \varepsilon\left(\|\mathrm{w}\|_{s}^{k_{0}, v}+\|\mathrm{w}\|_{s_{0}, v}^{k_{0}, v}\left\|\Im_{0}\right\|_{s+\sigma}^{k_{0}, v}\right) .
\end{aligned}
$$


Under the linear change of variables

$$
D G_{\delta}(\varphi, 0,0)\left(\begin{array}{c}
\widehat{\phi} \\
\widehat{y} \\
\widehat{\mathrm{w}}
\end{array}\right):=\left(\begin{array}{ccc}
\partial_{\phi} \theta_{0}(\varphi) & 0 & 0 \\
\partial_{\phi} I_{\delta}(\varphi) & {\left[\partial_{\phi} \theta_{0}(\varphi)\right]^{-\top}} & {\left[\left(\partial_{\theta} \widetilde{w}_{0}\right)\left(\theta_{0}(\varphi)\right)\right]^{\top} J_{\angle}^{-1}} \\
\partial_{\phi} w_{0}(\varphi) & 0 & \mathrm{Id}
\end{array}\right)\left(\begin{array}{c}
\widehat{\phi} \\
\widehat{y} \\
\widehat{\mathrm{w}}
\end{array}\right),
$$

the linearized operator $\mathrm{d}_{i, \alpha} \mathcal{F}\left(i_{\delta}\right)$ is approximately transformed into the one obtained when one linearizes the Hamiltonian system $(6.21)$ at $(\phi, y, \mathrm{w})=(\varphi, 0,0)$, differentiating also in $\alpha$ at $\alpha_{0}$ and changing $\partial_{t} \rightsquigarrow \omega \cdot \partial_{\varphi}$, namely

$$
\left(\begin{array}{c}
\widehat{\phi} \\
\widehat{y} \\
\widehat{\mathrm{w}} \\
\widehat{\alpha}
\end{array}\right) \mapsto\left(\begin{array}{c}
\omega \cdot \partial_{\varphi} \widehat{\phi}-\partial_{\phi} K_{10}(\varphi)[\widehat{\phi}]-\partial_{\alpha} K_{10}(\varphi)[\widehat{\alpha}]-K_{20}(\varphi) \widehat{y}-\left[K_{11}(\varphi)\right]^{\top} \widehat{\mathrm{w}} \\
\omega \cdot \partial_{\varphi} \widehat{y}+\partial_{\phi \phi} K_{00}(\varphi)[\widehat{\phi}]+\partial_{\alpha} \partial_{\phi} K_{00}(\varphi)[\widehat{\alpha}]+\left[\partial_{\phi} K_{10}(\varphi)\right]^{\top} \widehat{y}+\left[\partial_{\phi} K_{01}(\varphi)\right]^{\top} \widehat{\mathrm{w}} \\
\omega \cdot \partial_{\varphi} \widehat{\mathrm{w}}-J_{\angle}\left(\partial_{\phi} K_{01}(\varphi)[\widehat{\phi}]+\partial_{\alpha} K_{01}(\varphi)[\widehat{\alpha}]+K_{11}(\varphi) \widehat{y}+K_{02}(\varphi) \widehat{\mathrm{w}}\right)
\end{array}\right) .
$$

In order to construct an "almost approximate" inverse of (6.22), we need that

$$
\mathcal{L}_{\omega}:=\left.\Pi_{\mathbb{S}^{+}, \Sigma}^{<}\left(\omega \cdot \partial_{\varphi}-J K_{02}(\varphi)\right)\right|_{\mathfrak{H}_{\mathbb{S}^{+}, \Sigma}^{<}}
$$

is "almost invertible" (on traveling waves) up to remainders of size $O\left(N_{n-1}^{-\mathrm{a}}\right)$, where, for $n \in \mathbb{N}_{0}$

$$
N_{n}:=K_{n}^{p}, \quad K_{n}:=K_{0}^{\chi^{n}}, \quad \chi=3 / 2 .
$$

The $\left(K_{n}\right)_{n \geq 0}$ is the scale used in the nonlinear Nash-Moser iteration of Section 9 and $\left(N_{n}\right)_{n \geqq 0}$ is the one in the reducibility scheme of Section 8 . Let $H^{s}\left(\mathbb{T}^{\nu+1}\right):=$ $H^{s}\left(\mathbb{T}^{\nu+1}\right) \cap \mathfrak{H}_{\mathbb{S}^{+}, \Sigma}^{\angle}$.

(AI) Almost invertibility of $\mathcal{L}_{\omega}$ : There exist positive real numbers $\sigma, \mu(\mathrm{b}), \mathrm{a}, p$, $K_{0}$ and a subset $\Lambda_{o} \subset \mathrm{DC}(v, \tau) \times\left[\kappa_{1}, \kappa_{2}\right]$ such that, for all $(\omega, \kappa) \in \Lambda_{o}$, the operator $\mathcal{L}_{\omega}$ may be decomposed as

$$
\mathcal{L}_{\omega}=\mathcal{L}_{\omega}^{<}+\mathcal{R}_{\omega}+\mathcal{R}_{\omega}^{\perp},
$$

where, for every traveling wave function $g \in H_{\angle}^{s+\sigma}\left(\mathbb{T}^{\nu+1}, \mathbb{R}^{2}\right)$ and for every $(\omega, \kappa) \in \Lambda_{o}$, there is a traveling wave solution $h \in H^{s}\left(\mathbb{T}^{\nu+1}, \mathbb{R}^{2}\right)$ of $\mathcal{L}_{\omega}^{<} h=g$ satisfying, for all $s_{0} \leqq s \leqq S$,

$$
\left\|\left(\mathcal{L}_{\omega}^{<}\right)^{-1} g\right\|_{s}^{k_{0}, v} \lesssim S v^{-1}\left(\|g\|_{s+\sigma}^{k_{0}, v}+\|g\|_{s_{0}+\sigma}^{k_{0}, v}\left\|\Im_{0}\right\|_{S+\mu(\mathrm{b})+\sigma}^{k_{0}, v}\right) .
$$

In addition, if $g$ is anti-reversible, then $h$ is reversible. Moreover, for any $s_{0} \leqq$ $s \leqq S$, for any traveling wave $h \in \mathfrak{H}_{\mathbb{S}^{+}, \Sigma}^{\perp}$, the operators $\mathcal{R}_{\omega}, \mathcal{R}_{\omega}^{\perp}$ satisfy the estimates

$$
\begin{aligned}
& \left\|\mathcal{R}_{\omega} h\right\|_{s}^{k_{0}, v} \lesssim s \varepsilon v^{-1} N_{n-1}^{-\mathrm{a}}\left(\|h\|_{s+\sigma}^{k_{0}, v}+\|h\|_{s_{0}+\sigma}^{k_{0}, v}\left\|\Im_{0}\right\|_{s+\mu(\mathrm{b})+\sigma}^{k_{0}, v}\right), \\
& \left\|\mathcal{R}_{\omega}^{\perp} h\right\|_{s_{0}}^{k_{0}, v} \lesssim S K_{n}^{-b}\left(\|h\|_{S_{0}+b+\sigma}^{k_{0}, v}+\|h\|_{s_{0}+\sigma}^{k_{0}, v}\left\|\mathfrak{I}_{0}\right\|_{S_{0}+\mu(\mathrm{b})+\sigma+b}\right), \forall b>0, \\
& \left\|\mathcal{R}_{\omega}^{\perp} h\right\|_{S}^{k_{0}, v} \lesssim S\|h\|_{s+\sigma}^{k_{0}, v}+\|h\|_{s_{0}+\sigma}^{k_{0}, v}\left\|\Im_{0}\right\|_{s+\mu(\mathrm{b})+\sigma}^{k_{0}, v} .
\end{aligned}
$$


This assumption shall be verified by Theorem 8.10 at each $n$-th step of the NashMoser nonlinear iteration.

In order to find an almost approximate inverse of the linear operator in (6.22) (and so of $\mathrm{d}_{i, \alpha} \mathcal{F}\left(i_{\delta}\right)$ ), it is sufficient to invert the operator

$$
\mathbb{D}[\widehat{\phi}, \widehat{y}, \widehat{\mathrm{w}}, \widehat{\alpha}]:=\left(\begin{array}{c}
\omega \cdot \partial_{\varphi} \widehat{\phi}-\partial_{\alpha} K_{10}(\varphi)[\widehat{\alpha}]-K_{20}(\varphi) \widehat{y}-K_{11}^{\top}(\varphi) \widehat{\mathrm{w}} \\
\omega \cdot \partial_{\varphi} \widehat{y}+\partial_{\alpha} \partial_{\phi} K_{00}(\varphi)[\widehat{\alpha}] \\
\mathcal{L}_{\omega}^{<\widehat{\mathrm{w}}}-J_{\angle}\left(\partial_{\alpha} K_{01}(\varphi)[\widehat{\alpha}]+K_{11}(\varphi) \widehat{y}\right)
\end{array}\right)
$$

obtained neglecting in (6.22) the terms $\partial_{\phi} K_{10}, \partial_{\phi \phi} K_{00}, \partial_{\phi} K_{00}, \partial_{\phi} K_{01}$ (they vanish at an exact solution by Lemma 6.4) and the small remainders $\mathcal{R}_{\omega}, \mathcal{R}_{\omega}^{\perp}$ appearing in (6.25). We look for an inverse of $\mathbb{D}$ by solving the system

$$
\mathbb{D}[\widehat{\phi}, \widehat{y}, \widehat{\mathrm{w}}, \widehat{\alpha}]=\left(\begin{array}{l}
g_{1} \\
g_{2} \\
g_{3}
\end{array}\right),
$$

where $\left(g_{1}, g_{2}, g_{3}\right)$ is an anti-reversible traveling wave variation (cfr. Definition $3.33)$, that is

$g_{1}(\varphi)=g_{1}(-\varphi), \quad g_{2}(\varphi)=-g_{2}(-\varphi), \quad \mathcal{S}_{3}(\varphi)=-g_{3}(-\varphi)$,

$g_{1}(\varphi)=g_{1}(\varphi-\vec{\jmath} \varsigma), \quad g_{2}(\varphi)=g_{2}(\varphi-\vec{\jmath} \varsigma), \quad \tau_{\varsigma} g_{3}(\varphi)=g_{3}(\varphi-\vec{\jmath} \varsigma), \forall \varsigma \in \mathbb{R}$.

We first consider the second equation in (6.27)-(6.28), that is $\omega \cdot \partial_{\varphi} \widehat{y}=g_{2}-$ $\partial_{\alpha} \partial_{\phi} K_{00}(\varphi)[\widehat{\alpha}]$. By (6.29) and (6.19), the right hand side of this equation is odd in $\varphi$. In particular, this has zero average, and so

$$
\widehat{y}:=\left(\omega \cdot \partial_{\varphi}\right)^{-1}\left(g_{2}-\partial_{\alpha} \partial_{\phi} K_{00}(\varphi)[\widehat{\alpha}]\right) .
$$

Since $g_{2}(\varphi)=g_{2}(\varphi-\vec{\jmath} \varsigma)$ for any $\varsigma \in \mathbb{R}$ by $(6.30)$ and $\partial_{\alpha} \partial_{\phi} K_{00}(\varphi)[\widehat{\alpha}]$ satisfies the same property by $(6.20)$, we also deduce that

$$
\widehat{y}(\varphi-\vec{\jmath} \varsigma)=\widehat{y}(\varphi), \quad \forall \varsigma \in \mathbb{R} .
$$

Next we consider the third equation $\mathcal{L}_{\omega}^{<} \widehat{\mathrm{w}}=g_{3}+J_{\angle}\left(\partial_{\alpha} K_{01}(\varphi)[\widehat{\alpha}]+K_{11}(\varphi) \widehat{y}\right)$. The right hand side of this equation is a traveling wave by (6.30), (6.20), (6.32) and since $J_{\angle}=\Pi_{\mathbb{S}^{+}, \Sigma}^{\angle} J_{\mid \mathfrak{H}_{\mathbb{S}^{+}, \Sigma}^{<}}$commutes with $\tau_{\varsigma}$ (by Lemma 3.31). Thus, by assumption (AI), there is a traveling wave solution

$$
\widehat{\mathrm{W}}:=\left(\mathcal{L}_{\omega}^{<}\right)^{-1}\left(g_{3}+J_{\angle}\left(\partial_{\alpha} K_{01}(\varphi)[\widehat{\alpha}]+K_{11}(\varphi) \widehat{y}\right)\right) .
$$

Finally, we solve the first equation in (6.28), which, inserting (6.31) and (6.33), becomes

$$
\omega \cdot \partial_{\varphi} \widehat{\phi}=g_{1}+M_{1}(\varphi)[\widehat{\alpha}]+M_{2}(\varphi) g_{2}+M_{3}(\varphi) g_{3},
$$

where

$$
\begin{aligned}
& M_{1}(\varphi):=\partial_{\alpha} K_{10}(\varphi)-M_{2}(\varphi) \partial_{\alpha} \partial_{\phi} K_{00}(\varphi)+M_{3}(\varphi) J_{\angle} \partial_{\alpha} K_{01}(\varphi), \\
& M_{2}(\varphi):=K_{20}(\varphi)\left(\omega \cdot \partial_{\varphi}\right)^{-1}+K_{11}^{\top}(\varphi)\left(\mathcal{L}_{\omega}^{<}\right)^{-1} J_{\angle} K_{11}(\varphi)\left(\omega \cdot \partial_{\varphi}\right)^{-1},
\end{aligned}
$$




$$
M_{3}(\varphi):=K_{11}^{\top}(\varphi)\left(\mathcal{L}_{\omega}^{<}\right)^{-1}
$$

In order to solve (6.34), we choose $\widehat{\alpha}$ such that the average in $\varphi$ of the right hand side is zero. By Lemma 6.4 and (6.1), the $\varphi$-average of the matrix $M_{1}$ satisfies $\left\langle M_{1}\right\rangle_{\varphi}=\mathrm{Id}+O\left(\varepsilon v^{-1}\right)$. Then, for $\varepsilon v^{-1}$ small enough, $\left\langle M_{1}\right\rangle_{\varphi}$ is invertible and $\left\langle M_{1}\right\rangle_{\varphi}^{-1}=\mathrm{Id}+O\left(\varepsilon v^{-1}\right)$. Thus we define

$$
\widehat{\alpha}:=-\left\langle M_{1}\right\rangle_{\varphi}^{-1}\left(\left\langle g_{1}\right\rangle_{\varphi}+\left\langle M_{2} g_{2}\right\rangle_{\varphi}+\left\langle M_{3} g_{3}\right\rangle_{\varphi}\right),
$$

and the solution of equation (6.34)

$$
\widehat{\phi}:=\left(\omega \cdot \partial_{\varphi}\right)^{-1}\left(g_{1}+M_{1}(\varphi)[\widehat{\alpha}]+M_{2}(\varphi) g_{2}+M_{3}(\varphi) g_{3}\right) .
$$

Finally the property $\widehat{\phi}(\varphi-\vec{\jmath} \varsigma)=\widehat{\phi}(\varphi)$ for any $\varsigma \in \mathbb{R}$ follows by (6.20), (6.32) and the fact that $\widehat{\mathrm{w}}$ in $(6.33)$ is a traveling wave. This proves that $(\widehat{\phi}, \widehat{y}, \widehat{\mathrm{w}})$ is a traveling wave variation, that is (6.30) holds. Moreover, using (6.29), (6.19), Lemma 3.23, the fact that $J$ and $\mathcal{S}$ anti-commutes and $(\mathrm{AI})$, one checks that $(\widehat{\phi}, \widehat{y}, \widehat{\mathrm{w}})$ is reversible, that is

$$
\widehat{\phi}(\varphi)=-\widehat{\phi}(-\varphi), \quad \widehat{y}(\varphi)=\widehat{y}(-\varphi), \quad \mathcal{S} \widehat{\mathrm{W}}(\varphi)=\widehat{\mathrm{w}}(-\varphi) .
$$

In conclusion, we have obtained a solution $(\widehat{\phi}, \widehat{y}, \widehat{\mathrm{w}}, \widehat{\alpha})$ of the linear system (6.28), and, denoting the norm $\|(\phi, y, \mathrm{w}, \alpha)\|_{s}^{k_{0}, v}:=\max \left\{\|(\phi, y, \mathrm{w})\|_{s}^{k_{0}, v},|\alpha|^{k_{0}, v}\right\}$, we have

Proposition 6.5. Assume (6.1) (with $\mu=\mu(\mathrm{b})+\sigma$ ) and (AI). Then, for all $(\omega, \kappa) \in \Lambda_{o}$, for any anti-reversible traveling wave variation $g=\left(g_{1}, g_{2}, g_{3}\right)$ (that is satisfying (6.29)-(6.30)), system (6.28) has a solution $\mathbb{D}^{-1} g:=(\widehat{\phi}, \widehat{y}, \widehat{\mathrm{w}}, \widehat{\alpha})$, with $(\widehat{\phi}, \widehat{y}, \widehat{\mathrm{w}}, \widehat{\alpha})$ defined in (6.36),(6.31),(6.33),(6.35), where $(\widehat{\phi}, \widehat{y}, \widehat{\mathrm{w}})$ is a reversible traveling wave variation, satisfying, for any $s_{0} \leqq s \leqq S$

$$
\left\|\mathbb{D}^{-1} g\right\|_{S}^{k_{0}, v} \lesssim S v^{-1}\left(\|g\|_{s+\sigma}^{k_{0}, v}+\left\|\Im_{0}\right\|_{S+\mu(\mathrm{b})+\sigma}^{k_{0}, v}\|g\|_{s_{0}+\sigma}^{k_{0}, v}\right) .
$$

Proof. The estimate (6.38) follows by the explicit expression of the solution in (6.31), (6.33), (6.35), (6.36), and Lemma 6.4, (6.26), (6.1).

Finally we prove that the operator

$$
\mathbf{T}_{0}:=\mathbf{T}_{0}\left(i_{0}\right):=\left(D \widetilde{G}_{\delta}\right)(\varphi, 0,0) \circ \mathbb{D}^{-1} \circ\left(D G_{\delta}\right)(\varphi, 0,0)^{-1}
$$

is an almost approximate right inverse for $\mathrm{d}_{i, \alpha} \mathcal{F}\left(i_{0}\right)$, where $\widetilde{G}_{\delta}(\phi, y, \mathrm{w}, \alpha):=$ $\left(G_{\delta}(\phi, y, \mathrm{w}), \alpha\right)$ is the identity on the $\alpha$-component.

Theorem 6.6. (Almost approximate inverse) Assume (AI). Then there is $\bar{\sigma}:=$ $\bar{\sigma}\left(\tau, \nu, k_{0}\right)>0$ such that, if $(6.1)$ holds with $\mu=\mu(\mathrm{b})+\bar{\sigma}$, then, for all $(\omega, \kappa) \in$ $\Lambda_{o}$ and for any anti-reversible traveling wave variation $g:=\left(g_{1}, g_{2}, g_{3}\right)$ (that is satisfying (6.29)-(6.30)), the operator $\mathbf{T}_{0}$ defined in (6.39) satisfies, for all $s_{0} \leqq$ $s \leqq S$,

$$
\left\|\mathbf{T}_{0} g\right\|_{S}^{k_{0}, v} \lesssim_{S} v^{-1}\left(\|g\|_{s+\bar{\sigma}}^{k_{0}, v}+\left\|\Im_{0}\right\|_{S+\mu(\mathrm{b})+\bar{\sigma}}^{k_{0}, v}\|g\|_{s_{0}+\bar{\sigma}}^{k_{0}, v}\right) .
$$


Moreover, the first three components of $\mathbf{T}_{0} g$ form a reversible traveling wave variation (that is satisfy (6.37) and (6.30)). Finally, $\mathbf{T}_{0}$ is an almost approximate right inverse of $\mathrm{d}_{i, \alpha} \mathcal{F}\left(i_{0}\right)$, namely

$$
\mathrm{d}_{i, \alpha} \mathcal{F}\left(i_{0}\right) \circ \mathbf{T}_{0}-\mathrm{Id}=\mathcal{P}\left(i_{0}\right)+\mathcal{P}_{\omega}\left(i_{0}\right)+\mathcal{P}_{\omega}^{\perp}\left(i_{0}\right),
$$

where, for any traveling wave variation $g$, for all $s_{0} \leqq s \leqq S$,

$$
\begin{gathered}
\|\mathcal{P} g\|_{s}^{k_{0}, v} \lesssim S v^{-1}\left(\left\|\mathcal{F}\left(i_{0}, \alpha_{0}\right)\right\|_{s_{0}+\bar{\sigma}}^{k_{0}, v}\|g\|_{s+\bar{\sigma}}^{k_{0}, v}\right. \\
+\left(\left\|\mathcal{F}\left(i_{0}, \alpha_{0}\right)\right\|_{s+\bar{\sigma}}^{k_{0}, v}+\left\|\mathcal{F}\left(i_{0}, \alpha_{0}\right)\right\|_{s_{0}+\bar{\sigma}}^{k_{0}, v}\left\|\Im_{0}\right\|_{s+\mu(\mathrm{b})+\bar{\sigma}}^{k_{0}, v}\|g\|_{s_{0}+\bar{\sigma}}^{k_{0}, v}\right) \\
\left\|\mathcal{P}_{\omega} g\right\|_{S}^{k_{0}, v} \lesssim S \varepsilon v^{-2} N_{n-1}^{-\mathrm{a}}\left(\|g\|_{s+\bar{\sigma}}^{k_{0}, v}+\left\|\Im_{0}\right\|_{s+\mu(\mathrm{b})+\bar{\sigma}}^{k_{0}, v}\|g\|_{s_{0}+\bar{\sigma}}^{k_{0}, v}\right), \\
\left\|\mathcal{P}_{\omega}^{\perp} g\right\|_{s_{0}}^{k_{0}, v} \lesssim S, b v^{-1} K_{n}^{-b}\left(\|g\|_{s_{0}+\bar{\sigma}+b}^{k_{0}, v}+\left\|\Im_{0}\right\|_{s_{0}+\mu(\mathrm{b})+b+\bar{\sigma}}^{k_{0}, v}\|g\|_{s_{0}+\bar{\sigma}}^{k_{0}, v}\right), \quad \forall b>0 \\
\left\|\mathcal{P}_{\omega}^{\perp} g\right\|_{S}^{k_{0}, v} \lesssim S v^{-1}\left(\|g\|_{s+\bar{\sigma}}^{k_{0}, v}+\left\|\Im_{0}\right\|_{s+\mu(\mathrm{b})+\bar{\sigma}}^{k_{0}, v}\|g\|_{s_{0}+\bar{\sigma}}^{k_{0}, v}\right) .
\end{gathered}
$$

Proof. We claim that the first three components of $\mathbf{T}_{0} g$ form a reversible traveling wave variation. Indeed, differentiating (6.10) it follows that $D G_{\delta}(\varphi, 0,0)$, thus $\left(D G_{\delta}(\varphi, 0,0)\right)^{-1}$, is reversibility and momentum preserving (cfr. (3.54)). In particular these operators map an (anti)-reversible, respectively traveling, waves variation into a (anti)-reversible traveling waves variation (cfr. Lemma 3.34). Moreover, by Proposition 6.5 , the operator $\mathbb{D}^{-1}$ maps an anti-reversible traveling wave into a vector whose first three components form a reversible traveling wave. This proves the claim.

We now prove that the operators $\mathcal{P}, \mathcal{P}_{\omega}$ and $\mathcal{P} \perp \frac{\perp}{\omega}$ are defined on traveling waves. They are computed for example in Theorem 5.6 of [2]. To define them, introduce first the linear operators

$$
R_{Z}[\widehat{\phi}, \widehat{y}, \widehat{\mathrm{w}}, \widehat{\alpha}]:=\left(\begin{array}{c}
-\partial_{\phi} K_{10}\left(\varphi, \alpha_{0}\right)[\widehat{\phi}] \\
\partial_{\phi \phi} K_{00}\left(\varphi, \alpha_{0}\right)[\widehat{\phi}]+\left[\partial_{\phi} K_{10}\left(\varphi, \alpha_{0}\right)\right]^{\top} \widehat{y}+\left[\partial_{\phi} K_{01}\left(\varphi, \alpha_{0}\right)\right]^{\top} \widehat{\mathrm{w}} \\
-J_{\angle} \partial_{\phi} K_{01}\left(\varphi, \alpha_{0}\right)[\widehat{\phi}]
\end{array}\right)
$$

and

$$
\mathbb{R}_{\omega}[\widehat{\phi}, \widehat{y}, \widehat{\mathrm{w}}, \widehat{\alpha}]:=\left(\begin{array}{c}
0 \\
0 \\
\mathcal{R}_{\omega}[\widehat{\mathrm{w}}]
\end{array}\right), \quad \mathbb{R}_{\omega}^{\perp}[\widehat{\phi}, \widehat{y}, \widehat{\mathrm{w}}, \widehat{\alpha}]:=\left(\begin{array}{c}
0 \\
0 \\
\mathcal{R}_{\omega}^{\perp}[\widehat{\mathrm{w}}]
\end{array}\right) .
$$

Next, we denote by $\Pi$ the projection $(\widehat{\imath}, \widehat{\alpha}) \mapsto \widehat{\imath}$, by $\mathrm{u}_{\delta}(\varphi)=(\varphi, 0,0)$ the trivial torus, and by $\mathcal{E}, \mathcal{E}_{\omega}, \mathcal{E}_{\omega}^{\perp}$ the linear operators

$$
\begin{aligned}
\mathcal{E}:= & \mathrm{d}_{i, \alpha} \mathcal{F}\left(i_{0}\right)-\mathrm{d}_{i, \alpha} \mathcal{F}\left(i_{\delta}\right)+D^{2} G_{\delta}\left(\mathrm{u}_{\delta}\right)\left[D G_{\delta}\left(\mathrm{u}_{\delta}\right)^{-1} \mathcal{F}\left(i_{\delta}, \alpha_{0}\right), D G_{\delta}\left(\mathrm{u}_{\delta}\right)^{-1} \Pi[\cdot]\right] \\
& +D G_{\delta}\left(\mathrm{u}_{\delta}\right) R_{Z} D \widetilde{G}_{\delta}\left(\mathrm{u}_{\delta}\right)^{-1}, \\
\mathcal{E}_{\omega}: & =D G_{\delta}\left(\mathrm{u}_{\delta}\right) \mathbb{R}_{\omega} D \widetilde{G}_{\delta}\left(\mathrm{u}_{\delta}\right)^{-1}, \quad \mathcal{E}_{\omega}^{\perp}:=D G_{\delta}\left(\mathrm{u}_{\delta}\right) \mathbb{R}_{\omega}^{\perp} D \widetilde{G}_{\delta}\left(\mathrm{u}_{\delta}\right)^{-1} .
\end{aligned}
$$


It is then proved in Theorem 5.6 of [2] that $\mathcal{P}:=\mathcal{E} \circ \mathbf{T}_{0}, \mathcal{P}_{\omega}:=\mathcal{E}_{\omega} \circ \mathbf{T}_{0}, \mathcal{P}_{\omega}^{\perp}:=$ $\mathcal{E}_{\omega}^{\perp} \circ \mathbf{T}_{0}$. A direct inspection of these formulas shows that $\mathcal{P}, \mathcal{P} \omega$ and $\mathcal{P}{ }_{\omega}^{\perp}$ are defined on traveling wave variations. In particular, note that the operators $\mathbb{R}_{\omega}, \mathbb{R}_{\omega}^{\perp}$ in (6.45) are defined only if $\widehat{\mathrm{w}}$ is a traveling wave, because the operators $\mathcal{R}_{\omega}, \mathcal{R}_{\omega}^{\perp}$ defined in (AI) act only on a traveling wave. However, note that, if $g$ is a traveling wave variation, the third component of $D \widetilde{G}_{\delta}\left(u_{\delta}\right)^{-1} \mathbf{T}_{0} g$ is a traveling wave and therefore the operators $\mathcal{E}_{\omega}, \mathcal{E}_{\omega}^{\perp}$ in (6.46) are well defined.

The estimates (6.41)-(6.44) are proved as in Theorem 5.6 of [2], using Lemma 6.5 .

\section{The Linearized Operator in the Normal Subspace}

We now write an explicit expression of the linear operator $\mathcal{L}_{\omega}$ defined in (6.23).

Lemma 7.1. The Hamiltonian operator $\mathcal{L}_{\omega}$ defined in (6.23), acting on the normal subspace $\mathfrak{H}_{\mathbb{S}^{+}, \Sigma}^{<}$, has the form

$$
\mathcal{L}_{\omega}=\left.\Pi_{\mathbb{S}^{+}, \Sigma}^{<}(\mathcal{L}-\varepsilon J R)\right|_{\mathfrak{H}_{\mathbb{S}^{+}, \Sigma}^{<}}
$$

where :

1. $\mathcal{L}$ is the Hamiltonian operator

$$
\mathcal{L}:=\omega \cdot \partial_{\varphi}-J \partial_{u} \nabla_{u} \mathcal{H}\left(T_{\delta}(\varphi)\right),
$$

where $\mathcal{H}$ is the water waves Hamiltonian in the Wahlén variables defined in (2.13), evaluated at

$$
\begin{aligned}
T_{\delta}(\phi) & :=\varepsilon A\left(i_{\delta}(\phi)\right)=\varepsilon A\left(\theta_{0}(\phi), I_{\delta}(\phi), w_{0}(\phi)\right) \\
& =\varepsilon v^{\top}\left(\theta_{0}(\phi), I_{\delta}(\phi)\right)+\varepsilon w_{0}(\phi),
\end{aligned}
$$

the torus $i_{\delta}(\varphi):=\left(\theta_{0}(\varphi), I_{\delta}(\varphi), w_{0}(\varphi)\right)$ is defined in Lemma 6.2 and $A(\theta, I, w)$, $v^{\top}(\theta, I)$ in (2.50);

2. $R(\phi)$ has the finite rank form

$$
R(\phi)[h]=\sum_{j=1}^{v}\left(h, g_{j}\right)_{L^{2}} \chi_{j}, \quad \forall h \in \mathfrak{H}_{\mathbb{S}^{+}, \Sigma}^{L},
$$

for functions $g_{j}, \chi_{j} \in \mathfrak{H}_{\mathbb{S}^{+}, \Sigma}^{L}$ which satisfy, for some $\sigma:=\sigma\left(\tau, v, k_{0}\right)>0$, for all $j=1, \ldots, v$, for all $s \geqq s_{0}$,

$$
\begin{gathered}
\left\|g_{j}\right\|_{s}^{k_{0}, v}+\left\|\chi_{j}\right\|_{s}^{k_{0}, v} \lesssim_{s} 1+\left\|\Im_{\delta}\right\|_{s+\sigma}^{k_{0}, v}, \\
\left\|\mathrm{~d}_{i} g_{j}[\widehat{\imath}]\right\|_{s}+\left\|\mathrm{d}_{i} \chi_{j}[\widehat{\imath}]\right\|_{s} \lesssim_{s}\|\widehat{\imath}\|_{s+\sigma}+\|\widehat{\imath}\|_{s_{0}+\sigma}\left\|\Im_{\delta}\right\|_{s+\sigma} .
\end{gathered}
$$

The operator $\mathcal{L}_{\omega}$ is reversible and momentum preserving. 
Proof. In view of (6.18), (6.16) and (5.4) we have

$$
\begin{aligned}
K_{02}(\phi) & =\partial_{\mathrm{W}} \nabla_{\mathrm{W}} K_{\alpha}(\phi, 0,0)=\partial_{\mathrm{W}} \nabla_{\mathrm{W}}\left(H_{\alpha} \circ G_{\delta}\right)(\phi, 0,0) \\
& =\left.\Pi_{\angle}^{L^{2}} \boldsymbol{\Omega}_{W}\right|_{\mathfrak{H}_{\mathbb{S}^{+}, \Sigma}^{L}}+\varepsilon \partial_{\mathrm{W}} \nabla_{\mathrm{W}}\left(P \circ G_{\delta}\right)(\phi, 0,0),
\end{aligned}
$$

where $\boldsymbol{\Omega}_{W}$ is defined in (2.19) and $G_{\delta}$ in (6.9). Differentiating with respect to w the Hamiltonian

$$
\left(P \circ G_{\delta}\right)(\phi, y, \mathrm{w})=P\left(\theta_{0}(\phi), I_{\delta}(\phi)+L_{1}(\phi) y+L_{2}(\phi) \mathrm{w}, w_{0}(\phi)+\mathrm{w}\right),
$$

where $L_{1}(\phi):=\left[\partial_{\phi} \theta_{0}(\phi)\right]^{-\top}$ and $L_{2}(\phi):=\left[\partial_{\phi} \widetilde{w}_{0}\left(\theta_{0}(\phi)\right)\right]^{\top} J_{L}^{-1}$ (see (6.9)), we get

$$
\partial_{\mathrm{W}} \nabla_{\mathrm{W}}\left(P \circ G_{\delta}\right)(\phi, 0,0)=\partial_{w} \nabla_{w} P\left(i_{\delta}(\phi)\right)+R(\phi),
$$

where $R(\phi):=R_{1}(\phi)+R_{2}(\phi)+R_{3}(\phi)$ and

$$
\begin{aligned}
& R_{1}:=L_{2}(\phi)^{\top} \partial_{I}^{2} P\left(i_{\delta}(\phi)\right) L_{2}(\phi), \quad R_{2}:=L_{2}(\phi)^{\top} \partial_{w} \partial_{I} P\left(i_{\delta}(\phi)\right), \\
& R_{3}:=\partial_{I} \nabla_{w} P\left(i_{\delta}(\phi)\right) L_{2}(\phi) .
\end{aligned}
$$

Each operator $R_{1}, R_{2}, R_{3}$ has the finite rank form (7.4) because it is the composition of at least one operator with finite rank $\mathbb{R}^{v}$ in the space variable (for more details see for example Lemma 6.1 in [6]) and the estimates (7.5) follow by Lemma 6.1. By (7.6), (7.7), (5.3), (5.2), (5.1), we obtain

$$
K_{02}(\phi)=\Pi_{\angle}^{L^{2}}\left(\partial_{u} \nabla_{u} \mathcal{H}\right)\left(\varepsilon A\left(i_{\delta}(\phi)\right)\right)_{\mid \mathfrak{H}_{\mathbb{S}^{+}, \Sigma}^{<}}+\varepsilon R(\phi) .
$$

In conclusion, by (7.8), Lemma 2.7, and since $T_{\delta}(\phi)=\varepsilon A\left(i_{\delta}(\phi)\right)$, we deduce that the operator $\mathcal{L}_{\omega}$ in (6.23) has the form (7.1)-(7.2). Finally the operator $\Pi_{\mathbb{S}^{+}, \Sigma}^{\angle} J K_{02}(\varphi)$ is reversible and momentum preserving, by (6.19), (6.20), Lemmata 3.23, 3.31, and the fact that $J$ commutes with $\tau_{\varsigma}$ and anti-commutes with $\mathcal{S}$.

We remark that $\mathcal{L}$ in (7.2) is obtained by linearizing the water waves Hamiltonian system (2.13), (2.14) in the Wahlén variables defined in (2.11) at the torus $u=$ $(\eta, \zeta)=T_{\delta}(\varphi)$ defined in (7.3) and changing $\partial_{t} \rightsquigarrow \omega \cdot \partial_{\varphi}$. This is equal to

$$
\mathcal{L}=\omega \cdot \partial_{\varphi}-W^{-1}(\mathrm{~d} X)\left(W T_{\delta}(\varphi)\right) W
$$

where $X$ is the water waves vector field on the right hand side of (1.3). The operator $\mathcal{L}$ acts on (a dense subspace) of the phase space $L_{0}^{2} \times \dot{L}^{2}$.

In order to compute $\mathrm{d} X$ we use the "shape derivative" formula, see for example [26],

$$
G^{\prime}(\eta)[\widehat{\eta}] \psi:=\lim _{\varepsilon \rightarrow 0} \frac{1}{\varepsilon}(G(\eta+\varepsilon \widehat{\eta}) \psi-G(\eta) \psi)=-G(\eta)(B \widehat{\eta})-\partial_{x}(V \widehat{\eta}),
$$

where

$$
B(\eta, \psi):=\frac{G(\eta) \psi+\eta_{x} \psi_{x}}{1+\eta_{x}^{2}}, \quad V(\eta, \psi):=\psi_{x}-B(\eta, \psi) \eta_{x} .
$$


It turns out that $(V, B)=\left(\Phi_{x}, \Phi_{y}\right)$ is the gradient of the generalized velocity potential defined in (1.2), evaluated at the free surface $y=\eta(x)$.

Using (7.9), (1.3), (7.10), (7.11), the operator $\mathcal{L}$ is

$$
\begin{aligned}
\mathcal{L}=\omega \cdot \partial_{\varphi} & +\left(\begin{array}{cc}
\partial_{x} \widetilde{V}+G(\eta) B & -G(\eta) \\
g-\kappa \partial_{x} c \partial_{x}+B \widetilde{V}_{x}+B G(\eta) B \widetilde{V} \partial_{x}-B G(\eta)
\end{array}\right) \\
& +\frac{\gamma}{2}\left(\begin{array}{cc}
-G(\eta) \partial_{x}^{-1} & 0 \\
\partial_{x}^{-1} G(\eta) B-B G(\eta) \partial_{x}^{-1}-\frac{\gamma}{2} \partial_{x}^{-1} G(\eta) \partial_{x}^{-1}-\partial_{x}^{-1} G(\eta)
\end{array}\right),
\end{aligned}
$$

where

$$
\widetilde{V}:=V-\gamma \eta, \quad c(\eta):=\left(1+\eta_{x}^{2}\right)^{-\frac{3}{2}},
$$

and the functions $B:=B(\eta, \psi), V:=V(\eta, \psi), c:=c(\eta)$ in (7.12) are evaluated at the reversible traveling wave $(\eta, \psi):=W T_{\delta}(\varphi)$ where $T_{\delta}(\varphi)$ is defined in (7.3).

Remark 7.2. From now on we consider the operator $\mathcal{L}$ in (7.12) acting on (a dense subspace of) the whole $L^{2}(\mathbb{T}) \times L^{2}(\mathbb{T})$. In particular we extend the operator $\partial_{x}^{-1}$ to act on the whole $L^{2}(\mathbb{T})$ as in (3.20). In Sections 7.1-7.6 we are going to make several transformations, whose aim is to conjugate $\mathcal{L}$ to a constant coefficients Fourier multiplier, up to a pseudodifferential operator of order zero plus a remainder that satisfies tame estimates, both small in size, see $\mathcal{L}_{9}$ in (7.168). Finally, in Section 7.7 we shall conjugate the restricted operator $\mathcal{L}_{\omega}$ in (7.1).

Notation. In (7.12) and hereafter any function $a$ is identified with the corresponding multiplication operators $h \mapsto a h$, and, where there is no parenthesis, composition of operators is understood. For example, $\partial_{x} c \partial_{x}$ means: $h \mapsto \partial_{x}\left(c \partial_{x} h\right)$.

Lemma 7.3. The functions $(\eta, \zeta)=T_{\delta}(\varphi)$ and $B, \widetilde{V}, c$ defined in (7.11), (7.13) are quasi-periodic traveling waves. The functions $(\eta, \zeta)=T_{\delta}(\varphi)$ are (even $(\varphi, x)$, $\operatorname{odd}(\varphi, x)), B$ is $\operatorname{odd}(\varphi, x), \widetilde{V}$ is even $(\varphi, x)$ and $c$ is even $(\varphi, x)$. The Hamiltonian operator $\mathcal{L}$ is reversible and momentum preserving.

Proof. The function $(\eta, \zeta)=T_{\delta}(\varphi)$ is a quasi-periodic traveling wave and, using also Lemmata 3.32 and 3.26, we deduce that $B, \widetilde{V}, c$ are quasi-periodic traveling waves. Since $(\eta, \zeta)=T_{\delta}(\varphi)$ is reversible, we have that $(\eta, \zeta)$ is (even $(\varphi, x)$, $\operatorname{odd}(\varphi, x))$. Therefore, using also (2.6), we deduce that $B$ is odd $(\varphi, x), \widetilde{V}$ is even $(\varphi, x)$ and $c$ is even $(\varphi, x)$. By Lemmata 3.22 and 3.27, the operator $\mathcal{L}$ in (7.9) evaluated at the reversible quasi-periodic traveling wave $W T_{\delta}(\varphi)$ is reversible and momentum preserving.

For the sequel we will always assume the following ansatz (satisfied by the approximate solutions obtained along the nonlinear Nash-Moser iteration of Section 9): for some constants $\mu_{0}:=\mu_{0}(\tau, v)>0, v \in(0,1)$, (cfr. Lemma 6.2)

$$
\left\|\Im_{0}\right\|_{s_{0}+\mu_{0}}^{k_{0}, v},\left\|\Im_{\delta}\right\|_{s_{0}+\mu_{0}}^{k_{0}, v} \leqq 1 .
$$

In order to estimate the variation of the eigenvalues with respect to the approximate invariant torus, we need also to estimate the variation with respect to the torus $i(\varphi)$ in another low norm \|\|$_{s_{1}}$ for all Sobolev indexes $s_{1}$ such that

$$
s_{1}+\sigma_{0} \leqq s_{0}+\mu_{0}, \quad \text { for some } \sigma_{0}:=\sigma_{0}(\tau, v)>0 .
$$


Thus, by (7.14), we have

$$
\left\|\mathfrak{I}_{0}\right\|_{s_{1}+\sigma_{0}}^{k_{0}, v},\left\|\mathfrak{I}_{\delta}\right\|_{s_{1}+\sigma_{0}}^{k_{0}, v} \leqq 1
$$

The constants $\mu_{0}$ and $\sigma_{0}$ represent the loss of derivatives accumulated along the reduction procedure of the next sections. What is important is that they are independent of the Sobolev index $s$. In the following sections we shall denote by $\sigma:=\sigma\left(\tau, v, k_{0}\right)>0, \sigma_{N}\left(q_{0}\right):=\sigma_{N}\left(q_{0}, \tau, v, k_{0}\right), \sigma_{M}:=\sigma_{M}\left(k_{0}, \tau, v\right)>0$, $\aleph_{M}(\alpha)$ constants (which possibly increase from lemma to lemma) representing losses of derivatives along the finitely many steps of the reduction procedure.

Remark 7.4. In the next sections $\mu_{0}:=\mu_{0}(\tau, v, M, \alpha)>0$ will depend also on indexes $M, \alpha$, whose maximal values will be fixed depending only on $\tau$ and $\nu$ (and $k_{0}$ which is however considered an absolute constant along the paper). In particular $M$ is fixed in (8.5), whereas the maximal value of $\alpha$ depends on $M$, as explained in Remark 7.14.

As a consequence of Moser composition Lemma 3.2 and (6.4), the Sobolev norm of the function $u=T_{\delta}(\varphi)$ defined in (7.3) satisfies for all $s \geqq s_{0}$

$$
\|u\|_{s}^{k_{0}, v}=\|\eta\|_{s}^{k_{0}, v}+\|\zeta\|_{s}^{k_{0}, v} \leqq \varepsilon C(s)\left(1+\left\|\Im_{0}\right\|_{s}^{k_{0}, v}\right)
$$

(the map $A$ defined in (2.50) is smooth). Similarly, using (6.7),

$$
\left\|\Delta_{12} u\right\|_{s_{1}} \lesssim_{s_{1}} \varepsilon\left\|i_{2}-i_{1}\right\|_{s_{1}}, \quad \text { where } \Delta_{12} u:=u\left(i_{2}\right)-u\left(i_{1}\right) .
$$

We finally recall that $\mathfrak{I}_{0}=\mathfrak{I}_{0}(\omega, \kappa)$ is defined for all $(\omega, \kappa) \in \mathbb{R}^{v} \times\left[\kappa_{1}, \kappa_{2}\right]$ and that the functions $B, \widetilde{V}$ and $c$ appearing in $\mathcal{L}$ in (7.12) are $\mathcal{C}^{\infty}$ in $(\varphi, x)$, as $u=(\eta, \zeta)=T_{\delta}(\varphi)$ is.

\subsection{Quasi-periodic Reparametrization of Time}

We conjugate the operator $\mathcal{L}$ in (7.12) by the change of variables induced by the quasi-periodic reparametrization of time

$$
\vartheta:=\varphi+\omega p(\varphi) \Leftrightarrow \varphi=\vartheta+\omega \breve{p}(\vartheta)
$$

where $p(\varphi)$ is the real $\mathbb{T}^{\nu}$-periodic function defined in (7.87). Since $\eta(\varphi, x)$ is a quasi-periodic traveling wave, even in $(\varphi, x)$ (cfr. Lemma 7.3), it results that

$$
p(\varphi-\vec{\jmath} \varsigma)=p(\varphi), \forall \varsigma \in \mathbb{R}, \quad p \text { is } \operatorname{odd}(\varphi) .
$$

Moreover, by (7.87), (3.11), Lemma 3.2, (7.16) and (7.14) and Lemma 2.30 in [6], both $p$ and $\breve{p}$ satisfy, for some $\sigma:=\sigma\left(\tau, v, k_{0}\right)>0$, the tame estimates, for $s \geqq s_{0}$,

$$
\|p\|_{s}^{k_{0}, v}+\|\breve{p}\|_{s}^{k_{0}, v} \lesssim s \varepsilon^{2} v^{-1}\left(1+\left\|\Im_{0}\right\|_{s+\sigma}^{k_{0}, v}\right) .
$$


Remark 7.5. We perform as a first step the time reparametrization (7.17) of $\mathcal{L}$, with a function $p(\varphi)$ which will be fixed only later in Step 4 of Section 7.3, to avoid otherwise a technical difficulty in the conjugation of the remainders obtained by the Egorov theorem in Step 1 of Section 7.3. We need indeed to apply the Egorov Proposition 3.9 for conjugating the additional pseudodifferential term in (7.12) due to vorticity.

\section{Denoting by}

$$
(\mathcal{P} h)(\varphi, x):=h(\varphi+\omega p(\varphi), x), \quad\left(\mathcal{P}^{-1} h\right)(\vartheta, x):=h(\vartheta+\omega \breve{p}(\vartheta), x),
$$

the induced diffeomorphism of functions $h(\varphi, x) \in \mathbb{C}^{2}$, we have

$$
\mathcal{P}^{-1} \circ \omega \cdot \partial_{\varphi} \circ \mathcal{P}=\rho(\vartheta) \omega \cdot \partial_{\vartheta}, \quad \rho(\vartheta):=\mathcal{P}^{-1}\left(1+\omega \cdot \partial_{\varphi} p\right) .
$$

Therefore, for any $\omega \in \operatorname{DC}(v, \tau)$, we get

$$
\begin{aligned}
\mathcal{L}_{0}:= & \frac{1}{\rho} \mathcal{P}^{-1} \mathcal{L} \mathcal{P}=\omega \cdot \partial_{\vartheta}+\frac{1}{\rho}\left(\begin{array}{cc}
\partial_{x} \widetilde{V}+G(\eta) B & -G(\eta) \\
g-\kappa \partial_{x} c \partial_{x}+B \widetilde{V}_{x}+B G(\eta) B \widetilde{V} \partial_{x}-B G(\eta)
\end{array}\right) \\
+ & \frac{1}{\rho} \frac{\gamma}{2}\left(\begin{array}{c}
0 \\
\partial_{x}^{-1} G(\eta) B-B(\eta) \partial_{x}^{-1}
\end{array}\right), \quad(7.21)
\end{aligned}
$$

where $\tilde{V}, B, c, V$ and $G(\eta)$ are evaluated at $\left(\eta_{p}, \psi_{p}\right):=\mathcal{P}^{-1}(\eta, \psi)$. For simplicity in the notation we do not report in (7.21) the explicit dependence on $p$, writing for example (cfr. (7.13))

$$
c=\left(1+\left(\mathcal{P}^{-1} \eta\right)_{x}^{2}\right)^{-\frac{3}{2}}=\mathcal{P}^{-1}\left(1+\eta_{x}^{2}\right)^{-\frac{3}{2}} .
$$

Lemma 7.6. The maps $\mathcal{P}, \mathcal{P}^{-1}$ are $\mathcal{D}^{k_{0}}-\left(k_{0}+1\right)$-tame, the maps $\mathcal{P}-\operatorname{Id}$ and $\mathcal{P}^{-1}-\mathrm{Id}$ are $\mathcal{D}^{k_{0}}-\left(k_{0}+2\right)$-tame, with tame constants satisfying, for some $\sigma:=\sigma\left(\tau, v, k_{0}\right)>$ 0 and for any $s_{0} \leqq s \leqq S$,

$$
\mathfrak{M}_{\mathcal{P}^{ \pm 1}}(s) \lesssim s 1+\left\|\Im_{0}\right\|_{s+\sigma}^{k_{0}, v}, \quad \mathfrak{M}_{\mathcal{P}^{ \pm 1}-\mathrm{Id}}(s) \lesssim s \varepsilon^{2} v^{-1}\left(1+\left\|\mathfrak{I}_{0}\right\|_{s+\sigma}^{k_{0}, v}\right)
$$

The function $\rho$ defined in (7.20) satisfies

$$
\rho \text { is even }(\vartheta) \text { and } \rho(\vartheta-\vec{\jmath} \varsigma)=\rho(\vartheta), \forall \varsigma \in \mathbb{R} \text {. }
$$

The operator $\mathcal{L}_{0}$ is Hamiltonian, reversible and momentum preserving.

Proof. Estimates (7.23) follow by (7.19) and Lemma 2.30 in [6], writing ( $\mathcal{P}-$ Id $h=p \int_{0}^{1} \mathcal{P}_{\tau}\left(\omega \cdot \partial_{\varphi} h\right) \mathrm{d} \tau$, where $\left(\mathcal{P}_{\tau} h\right)(\varphi, x):=h(\varphi+\tau \omega p(\varphi), x)$. We deduce (7.24) by (7.18) and (7.20). Denoting $\mathcal{L}=\omega \cdot \partial_{\varphi}+A(\varphi)$ the operator $\mathcal{L}$ in (7.12), then the operator $\mathcal{L}_{0}$ in $(7.21)$ is $\mathcal{L}_{0}=\omega \cdot \partial_{\vartheta}+A_{+}(\vartheta)$ with $A_{+}(\vartheta)=\rho^{-1}(\vartheta) A(\vartheta+$ $\breve{p}(\vartheta) \omega)$. It follows that $A_{+}(\varphi)$ is Hamiltonian, reversible and momentum preserving as $A(\varphi)$ (Lemma 7.3).

Remark 7.7. The map $\mathcal{P}$ is not reversibility and momentum preserving according to Definitions 3.17 and, respectively, 3.24, but maps (anti)-reversible, respectively traveling, waves, into (anti)-reversible, respectively traveling, waves. Note that the multiplication operator for the function $\rho(\vartheta)$, which satisfies (7.24), is reversibility and momentum preserving according to Definitions 3.17 and 3.24. 


\subsection{Linearized Good Unknown of Alinhac}

We conjugate the linear operator $\mathcal{L}_{0}$ in $(7.21)$, where we rename $\vartheta$ with $\varphi$, by the multiplication matrix operator

$$
\mathcal{Z}:=\left(\begin{array}{cc}
\text { Id } & 0 \\
B & \text { Id }
\end{array}\right), \quad \mathcal{Z}^{-1}=\left(\begin{array}{cc}
\text { Id } & 0 \\
-B & \text { Id }
\end{array}\right)
$$

obtaining (in view of (3.46))

$$
\begin{aligned}
& \mathcal{L}_{1}:=\mathcal{Z}^{-1} \mathcal{L}_{0} \mathcal{Z} \\
& =\omega \cdot \partial_{\varphi}+\frac{1}{\rho}\left(\begin{array}{cc}
\partial_{x} \widetilde{V} & -G(\eta) \\
g+a-\kappa \partial_{x} c \partial_{x} & \widetilde{V} \partial_{x}
\end{array}\right)-\frac{1}{\rho} \frac{\gamma}{2}\left(\begin{array}{cc}
G(\eta) \partial_{x}^{-1} & 0 \\
\frac{\gamma}{2} \partial_{x}^{-1} G(\eta) \partial_{x}^{-1} & \partial_{x}^{-1} G(\eta)
\end{array}\right),
\end{aligned}
$$

where $a$ is the function

$$
a:=\widetilde{V} B_{x}+\rho\left(\omega \cdot \partial_{\varphi} B\right) .
$$

The matrix $\mathcal{Z}$ amounts to introduce, as in [26] and [2,6], a linearized version of the "good unknown of Alinhac".

Lemma 7.8. The maps $\mathcal{Z}^{ \pm 1}-\mathrm{Id}$ are $\mathcal{D}^{k_{0}}$-tame with tame constants satisfying, for some $\sigma:=\sigma\left(\tau, v, k_{0}\right)>0$, for all $s \geqq s_{0}$,

$$
\mathfrak{M}_{\mathcal{Z}^{ \pm 1}-\mathrm{Id}}(s), \mathfrak{M}_{\left(\mathcal{Z}^{ \pm 1}-\mathrm{Id}\right)^{*}}(s) \lesssim s \varepsilon\left(1+\left\|\mathfrak{I}_{0}\right\|_{s+\sigma}^{k_{0}, v}\right) .
$$

The function $a$ is a quasi-periodic traveling wave even $(\varphi, x)$. There is $\sigma:=$ $\sigma\left(\tau, v, k_{0}\right)>0$ such that, for all $s \geqq s_{0}$,

$$
\begin{aligned}
& \|a\|_{s}^{k_{0}, v}+\|\widetilde{V}\|_{s}^{k_{0}, v}+\|B\|_{s}^{k_{0}, v} \lesssim_{s} \varepsilon\left(1+\left\|\Im_{0}\right\|_{s+\sigma}^{k_{0}, v}\right), \\
& \|1-c\|_{s}^{k_{0}, v} \lesssim_{s} \varepsilon^{2}\left(1+\left\|\Im_{0}\right\|_{s+\sigma}^{k_{0}, v}\right) .
\end{aligned}
$$

Moreover, for any $s_{1}$ as in (7.15),

$$
\begin{aligned}
& \left\|\Delta_{12} a\right\|_{s_{1}}+\left\|\Delta_{12} \widetilde{V}\right\|_{s_{1}}+\left\|\Delta_{12} B\right\|_{s_{1}} \lesssim_{s_{1}} \varepsilon\left\|i_{1}-i_{2}\right\|_{s_{1}+\sigma}, \\
& \left\|\Delta_{12} c\right\|_{s_{1}} \lesssim_{s_{1}} \varepsilon^{2}\left\|i_{1}-i_{2}\right\|_{s_{1}+\sigma}, \\
& \left\|\Delta_{12}\left(\mathcal{Z}^{ \pm 1}\right) h\right\|_{s_{1}},\left\|\Delta_{12}\left(\mathcal{Z}^{ \pm 1}\right)^{*} h\right\|_{s_{1}} \lesssim_{s_{1}} \varepsilon\left\|i_{1}-i_{2}\right\|_{s_{1}+\sigma}\|h\|_{s_{1}} .
\end{aligned}
$$

The operator $\mathcal{L}_{1}$ is Hamiltonian, reversible and momentum preserving.

Proof. The estimates (7.28) follow by the expressions of $a, \widetilde{V}, B, c$ in (7.26), (7.11), (7.13), (reparametrized by $\mathcal{P}^{-1}$ as in (7.22)), Lemma 3.2 and (7.23), (3.7), (3.33), (3.37) and (3.36). The estimate (7.27) follows by (3.37), (3.22), (7.28) and since the adjoint $\mathcal{Z}^{*}=\left(\begin{array}{cc}\text { Id } & B \\ 0 & \mathrm{Id}\end{array}\right)$. The estimates (7.29)-(7.31) follow similarly. Since $B$ is a $\operatorname{odd}(\varphi, x)$ quasi-periodic traveling wave, then the operators $\mathcal{Z}^{ \pm}$are reversibility and momentum preserving. 


\subsection{Symmetrization and Reduction of the Highest Order}

The aim of this long section is to conjugate the Hamiltonian operator $\mathcal{L}_{1}$ in (7.25) to the Hamiltonian operator $\mathcal{L}_{5}$ in (7.89) whose coefficient $\mathrm{m}_{\frac{3}{2}}$ of the highest order is constant. This is achieved in several steps. All the transformations of this section are symplectic.

Recalling the expansion (3.32) of the Dirichlet-Neumann operator, we first write

$$
\begin{aligned}
\mathcal{L}_{1}= & \omega \cdot \partial_{\varphi}+\frac{1}{\rho}\left(\begin{array}{cc}
-\frac{\gamma}{2} G(0) \partial_{x}^{-1} & -G(0) \\
-\kappa \partial_{x} c \partial_{x}+g-\left(\frac{\gamma}{2}\right)^{2} \partial_{x}^{-1} G(0) \partial_{x}^{-1} & -\frac{\gamma}{2} \partial_{x}^{-1} G(0)
\end{array}\right) \\
& +\frac{1}{\rho}\left(\begin{array}{cc}
\partial_{x} \widetilde{V} & 0 \\
a & \widetilde{V} \partial_{x}
\end{array}\right)+\mathbf{R}_{1}
\end{aligned}
$$

where

$$
\mathbf{R}_{1}:=-\frac{1}{\rho}\left(\begin{array}{cc}
\frac{\gamma}{2} \mathcal{R}_{G}(\eta) \partial_{x}^{-1} & \mathcal{R}_{G}(\eta) \\
\left(\frac{\gamma}{2}\right)^{2} \partial_{x}^{-1} \mathcal{R}_{G}(\eta) \partial_{x}^{-1} & \frac{\gamma}{2} \partial_{x}^{-1} \mathcal{R}_{G}(\eta)
\end{array}\right)
$$

is a small remainder in $\operatorname{OPS}^{-\infty}$.

Step 1: We first conjugate $\mathcal{L}_{1}$ with the symplectic change of variable (cfr. (3.42))

$$
(\mathcal{E} u)(\varphi, x):=\sqrt{1+\beta_{x}(\varphi, x)}(\mathcal{B} u)(\varphi, x), \quad(\mathcal{B} u)(\varphi, x):=u(\varphi, x+\beta(\varphi, x)),
$$

induced by a family of $\varphi$-dependent diffeomorphisms of the torus $y=x+\beta(\varphi, x)$, where $\beta(\varphi, x)$ is a small function to be determined, see (7.68). We denote the inverse diffeomorphism by $x=y+\breve{\beta}(\varphi, y)$. By direct computation we have that

$$
\begin{aligned}
\mathcal{E}^{-1} \widetilde{V} \partial_{x} \mathcal{E} & =\left\{\mathcal{B}^{-1}\left(\widetilde{V}\left(1+\beta_{x}\right)\right)\right\} \partial_{y}+\frac{1}{2}\left\{\mathcal{B}^{-1} \widetilde{V} \beta_{x x}\left(1+\beta_{x}\right)^{-1}\right\} \\
\mathcal{E}^{-1} \partial_{x} \widetilde{V} \mathcal{E} & =\left\{\mathcal{B}^{-1}\left(\widetilde{V}\left(1+\beta_{x}\right)\right)\right\} \partial_{y}+\left\{\mathcal{B}^{-1}\left(\widetilde{V}_{x}+\frac{1}{2} \widetilde{V} \beta_{x x}\left(1+\beta_{x}\right)^{-1}\right)\right\} \\
\mathcal{E}^{-1} a \mathcal{E} & =\left\{\mathcal{B}^{-1} a\right\} \\
\mathcal{E}^{-1} \partial_{x} c \partial_{x} \mathcal{E} & =\mathcal{B}^{-1}\left(1+\beta_{x}\right)^{-\frac{1}{2}} \mathcal{B} \mathcal{B}^{-1} \partial_{x} \mathcal{B} \mathcal{B}^{-1} c \mathcal{B} \mathcal{B}^{-1} \partial_{x} \mathcal{B} \mathcal{B}^{-1}\left(1+\beta_{x}\right)^{\frac{1}{2}} \mathcal{B} \\
& =\left\{\mathcal{B}^{-1}\left(1+\beta_{x}\right)^{\frac{1}{2}}\right\} \partial_{y}\left\{\mathcal{B}^{-1}\left(c\left(1+\beta_{x}\right)\right)\right\} \partial_{y}\left\{\mathcal{B}^{-1}\left(1+\beta_{x}\right)^{\frac{1}{2}}\right\} \\
\mathcal{E}^{-1} \omega \cdot \partial_{\varphi} \mathcal{E} & =\omega \cdot \partial_{\varphi}+\left\{\mathcal{B}^{-1}\left(\omega \cdot \partial_{\varphi} \beta\right)\right\} \partial_{y}+\frac{1}{2}\left\{\mathcal{B}^{-1}\left(\left(\omega \cdot \partial_{\varphi} \beta_{x}\right)\left(1+\beta_{x}\right)^{-1}\right)\right\}
\end{aligned}
$$

Then we write the Dirichlet-Neumann operator $G(0)$ in (1.7) as

$$
G(0)=G(0, \mathrm{~h})=\partial_{x} \mathcal{H} T(\mathrm{~h}),
$$

where $\mathcal{H}$ is the Hilbert transform in (3.19) and

$$
T(\mathrm{~h}):= \begin{cases}\tanh (\mathrm{h}|D|)=\mathrm{Id}+\mathrm{Op}\left(r_{\mathrm{h}}\right) & \text { if } \mathrm{h}<+\infty, r_{\mathrm{h}}(\xi):=-\frac{2}{1+e^{2 \mathrm{~h}|\xi| \chi(\xi)}} \in S^{-\infty}, \\ \operatorname{Id} & \text { if } \mathrm{h}=\infty .\end{cases}
$$


We have the conjugation formula (see formula (7.42) in [2])

$$
\mathcal{B}^{-1} G(0) \mathcal{B}=\left\{\mathcal{B}^{-1}\left(1+\beta_{x}\right)\right\} G(0)+\mathcal{R}_{1},
$$

where

$$
\begin{aligned}
\mathcal{R}_{1}:= & \left\{\mathcal{B}^{-1}\left(1+\beta_{x}\right)\right\} \partial_{y}\left(\mathcal{H}\left(\mathcal{B}^{-1} \mathrm{Op}\left(r_{\mathrm{h}}\right) \mathcal{B}-\mathrm{Op}\left(r_{\mathrm{h}}\right)\right)\right. \\
& \left.+\left(\mathcal{B}^{-1} \mathcal{H} \mathcal{B}-\mathcal{H}\right)\left(\mathcal{B}^{-1} T(\mathrm{~h}) \mathcal{B}\right)\right) .
\end{aligned}
$$

The operator $\mathcal{R}_{1}$ is in OP $S^{-\infty}$ because both $\mathcal{B}^{-1} \mathrm{Op}\left(r_{\mathrm{h}}\right) \mathcal{B}-\mathrm{Op}\left(r_{\mathrm{h}}\right)$ and $\mathcal{B}^{-1} \mathcal{H} \mathcal{B}-\mathcal{H}$ are in OP $S^{-\infty}$ and there is $\sigma>0$ such that, for any $m \in \mathbb{N}, s \geqq s_{0}$, and $\alpha \in \mathbb{N}_{0}$,

$$
\begin{aligned}
& \left\|\mathcal{B}^{-1} \mathcal{H B}-\mathcal{H}\right\|_{-m, s, \alpha}^{k_{0}, v} \lesssim_{m, s, \alpha, k_{0}}\|\beta\|_{s+m+\alpha+\sigma}^{k_{0}, v}, \\
& \left\|\mathcal{B}^{-1} \mathrm{Op}\left(r_{\mathrm{h}}\right) \mathcal{B}-\mathrm{Op}\left(r_{\mathrm{h}}\right)\right\|_{-m, s, \alpha}^{k_{0}, v} \lesssim_{m, s, \alpha, k_{0}}\|\beta\|_{s+m+\alpha+\sigma}^{k_{0}, v} .
\end{aligned}
$$

The first estimate is given in Lemmata 2.36 and 2.32 in [6], whereas the second one follows by that fact that $r_{\mathrm{h}} \in S^{-\infty}$ (see (7.41)), Lemma 2.18 in [2] and Lemmata 2.34 and 2.32 in [6]. Therefore by (7.42) we obtain

$$
\mathcal{E}^{-1} G(0) \mathcal{E}=\left\{\mathcal{B}^{-1}\left(1+\beta_{x}\right)^{\frac{1}{2}}\right\} G(0)\left\{\mathcal{B}^{-1}\left(1+\beta_{x}\right)^{\frac{1}{2}}\right\}+\widetilde{\mathcal{R}}_{1},
$$

where

$$
\widetilde{\mathcal{R}}_{1}:=\left\{\mathcal{B}^{-1}\left(1+\beta_{x}\right)^{-\frac{1}{2}}\right\} \mathcal{R}_{1}\left\{\mathcal{B}^{-1}\left(1+\beta_{x}\right)^{\frac{1}{2}}\right\} .
$$

Next we transform $G(0) \partial_{x}^{-1}$. By (7.40) and using the identities $\mathcal{H} \partial_{x} \partial_{x}^{-1}=\mathcal{H}$ and $\mathcal{H} T(\mathrm{~h})=G(0) \partial_{y}^{-1}$ on the periodic functions, we have that

$$
\mathcal{E}^{-1} G(0) \partial_{x}^{-1} \mathcal{E}=\mathcal{E}^{-1} \partial_{x} \mathcal{H} T(\mathrm{~h}) \partial_{x}^{-1} \mathcal{E}=G(0) \partial_{y}^{-1}+\mathcal{R}_{2},
$$

where

$$
\begin{aligned}
\mathcal{R}_{2} & :=\left\{\mathcal{B}^{-1}\left(1+\beta_{x}\right)^{-\frac{1}{2}}\right\}\left[\mathcal{H} T(\mathrm{~h}),\left\{\mathcal{B}^{-1}\left(1+\beta_{x}\right)^{\frac{1}{2}}\right\}-1\right]+\left\{\mathcal{B}^{-1}\left(1+\beta_{x}\right)^{-\frac{1}{2}}\right\} \circ \\
& \circ\left(\left(\mathcal{B}^{-1} \mathcal{H B}-\mathcal{H}\right)\left(\mathcal{B}^{-1} T(\mathrm{~h}) \mathcal{B}\right)+\mathcal{H}\left(\mathcal{B}^{-1} \mathrm{Op}\left(r_{\mathrm{h}}\right) \mathcal{B}-\mathrm{Op}\left(r_{\mathrm{h}}\right)\right)\right)\left\{\mathcal{B}^{-1}\left(1+\beta_{x}\right)^{\frac{1}{2}}\right\} .
\end{aligned}
$$

The operator $\mathcal{R}_{2}$ is in OPS $S^{-\infty}$ by (7.44), (7.41) and because the commutator of $\mathcal{H}$ with any smooth function $a$ is in OP $S^{-\infty}$, in particular (see Lemma 2.35 of [6]) there is $\sigma>0$ such that, for any $m \in \mathbb{N}, s \geqq s_{0}$, and $\alpha \in \mathbb{N}_{0}$,

$$
\|[\mathcal{H} T(\mathrm{~h}), a]\|_{-m, s, \alpha}^{k_{0}, v} \lesssim m, s, \alpha, k_{0}\|a\|_{s+m+\alpha+\sigma}^{k_{0}, v} .
$$

Finally we conjugate $\partial_{x}^{-1} G(0) \partial_{x}^{-1}$. By the Egorov Proposition 3.9, we have that, for any $N \in \mathbb{N}$,

$$
\mathcal{E}^{-1} \partial_{x}^{-1} \mathcal{E}=\left\{\mathcal{B}^{-1}\left(\frac{1}{1+\beta_{x}}\right)\right\} \partial_{y}^{-1}+P_{-2, N}^{(1)}(\varphi, x, D)+\mathrm{R}_{N},
$$

where $P_{-2, N}^{(1)}(\varphi, x, D) \in \mathrm{OPS}^{-2}$ is

$$
P_{-2, N}^{(1)}(\varphi, x, D):=\left\{\mathcal{B}^{-1}\left(1+\beta_{x}\right)^{-\frac{1}{2}}\right\}\left\{\left[p_{-1} \partial_{y}^{-1}, \mathcal{B}^{-1}\left(1+\beta_{x}\right)^{\frac{1}{2}}\right]\right.
$$




$$
\left.+\sum_{j=1}^{N} p_{-1-j} \partial_{y}^{-1-j}\left\{\mathcal{B}^{-1}\left(1+\beta_{x}\right)^{\frac{1}{2}}\right\}\right\}
$$

with functions $p_{-1-j}(\lambda ; \varphi, y), j=0, \ldots, N$, satisfying (3.30) and $\mathrm{R}_{N}$ is a regularizing operator satisfying the estimate (3.31). So, using (7.50) and (7.47), we obtain

$$
\begin{aligned}
\mathcal{E}^{-1} \partial_{x}^{-1} G(0) \partial_{x}^{-1} \mathcal{E} & =\left(\mathcal{E}^{-1} \partial_{x}^{-1} \mathcal{E}\right)\left(\mathcal{E}^{-1} G(0) \partial_{x}^{-1} \mathcal{E}\right) \\
& =\partial_{y}^{-1} G(0) \partial_{y}^{-1}+P_{-1, N}^{(2)}+\mathrm{R}_{2, N}
\end{aligned}
$$

where

$$
P_{-1, N}^{(2)}:=\left(-\left\{\mathcal{B}^{-1}\left(\frac{\beta_{x}}{1+\beta_{x}}\right)\right\} \partial_{y}^{-1}+P_{-2, N}^{(1)}(\varphi, x, D)\right) G(0) \partial_{y}^{-1} \in \mathrm{OPS}^{-1}
$$

and $\mathrm{R}_{2, N}$ is the regularizing operator

$$
\mathrm{R}_{2, N}:=\left(\mathcal{E}^{-1} \partial_{x}^{-1} \mathcal{E}\right) \mathcal{R}_{2}+\mathrm{R}_{N} G(0) \partial_{y}^{-1} .
$$

The smoothing order $N \in \mathbb{N}$ will be chosen in Section 8 during the KAM iteration (see also Remark 7.11).

In conclusion, by (7.35)-(7.39), (7.45), (7.47) and (7.51) we obtain

$$
\begin{aligned}
\mathcal{L}_{2}:=\mathcal{E}^{-1} \mathcal{L}_{1} \mathcal{E}= & \omega \cdot \partial_{\varphi}+\frac{1}{\rho}\left(\begin{array}{cc}
-\frac{\gamma}{2} G(0) \partial_{y}^{-1} & -a_{2} G(0) a_{2} \\
-\kappa a_{2} \partial_{y} a_{3} \partial_{y} a_{2}+g-\left(\frac{\gamma}{2}\right)^{2} \partial_{y}^{-1} G(0) \partial_{y}^{-1}-\frac{\gamma}{2} \partial_{y}^{-1} G(0)
\end{array}\right) \\
& +\frac{1}{\rho}\left(\begin{array}{cc}
a_{1} \partial_{y}+a_{4} & 0 \\
a_{5}-\left(\frac{\gamma}{2}\right)^{2} P_{-1, N}^{(2)} & a_{1} \partial_{y}+a_{6}
\end{array}\right)+\mathbf{R}_{2}^{\Psi}+\mathbf{T}_{2, N}
\end{aligned}
$$

where

$$
\begin{aligned}
& a_{1}(\varphi, y):=\mathcal{B}^{-1}\left(\left(1+\beta_{x}\right) \widetilde{V}+\left(\omega \cdot \partial_{\varphi} \beta\right)\right), \\
& a_{2}(\varphi, y):=\mathcal{B}^{-1}\left(\sqrt{1+\beta_{x}}\right), \quad a_{3}(\varphi, y):=\mathcal{B}^{-1}\left(c\left(1+\beta_{x}\right)\right), \\
& a_{4}(\varphi, y):=\mathcal{B}^{-1}\left(\frac{\widetilde{V} \beta_{x x}+\left(\omega \cdot \partial_{\varphi} \beta_{x}\right)}{2\left(1+\beta_{x}\right)}+\widetilde{V}_{x}\right), \quad a_{5}(\varphi, y):=\mathcal{B}^{-1} a, \\
& a_{6}(\varphi, y):=\mathcal{B}^{-1}\left(\frac{\widetilde{V} \beta_{x x}+\left(\omega \cdot \partial_{\varphi} \beta_{x}\right)}{2\left(1+\beta_{x}\right)}\right),
\end{aligned}
$$

the operator $P_{-1, N}^{(2)} \in \mathrm{OPS}^{-1}$ is defined in (7.52) and

$$
\mathbf{R}_{2}^{\Psi}:=-\frac{1}{\rho}\left(\begin{array}{cc}
\frac{\gamma}{2} \mathcal{R}_{2} & \widetilde{\mathcal{R}}_{1} \\
0 & \frac{\gamma}{2} \mathcal{R}_{2}
\end{array}\right)+\mathcal{E}^{-1} \mathbf{R}_{1} \mathcal{E}, \quad \mathbf{T}_{2, N}:=-\frac{1}{\rho}\left(\frac{\gamma}{2}\right)^{2}\left(\begin{array}{cc}
0 & 0 \\
R_{2, N} & 0
\end{array}\right),
$$

with $\widetilde{\mathcal{R}}_{1}, \mathcal{R}_{2}, \mathrm{R}_{2, N}$ defined in (7.46), (7.48), (7.53) and $\mathbf{R}_{1}$ in (7.33). 
Step 2: We now conjugate the operator $\mathcal{L}_{2}$ in (7.54) with the multiplication matrix operator

$$
\mathcal{Q}:=\left(\begin{array}{cc}
q & 0 \\
0 & q^{-1}
\end{array}\right), \quad \mathcal{Q}^{-1}:=\left(\begin{array}{cc}
q^{-1} & 0 \\
0 & q
\end{array}\right)
$$

where $q(\varphi, y)$ is a real function, close to 1 , to be determined. The maps $\mathcal{Q}$ and $\mathcal{Q}^{-1}$ are symplectic (cfr. (3.42)). We have that

$$
\mathcal{L}_{3}:=\mathcal{Q}^{-1} \mathcal{L}_{2} \mathcal{Q}=\omega \cdot \partial_{\varphi}+\frac{1}{\rho}\left(\begin{array}{ll}
A & B \\
C & D
\end{array}\right)+\mathcal{Q}^{-1}\left(\mathbf{R}_{2}^{\Psi}+\mathbf{T}_{2, N}\right) \mathcal{Q},
$$

where

$$
\begin{aligned}
A & :=q^{-1}\left(-\frac{\gamma}{2} G(0) \partial_{y}^{-1}+a_{1} \partial_{y}+a_{4}\right) q+\rho q^{-1}\left(\omega \cdot \partial_{\varphi} q\right) \\
B & :=-q^{-1} a_{2} G(0) a_{2} q^{-1} \\
C & :=q\left(-\kappa a_{2} \partial_{y} a_{3} \partial_{y} a_{2}+g-\left(\frac{\gamma}{2}\right)^{2} \partial_{y}^{-1} G(0) \partial_{y}^{-1}+a_{5}-\left(\frac{\gamma}{2}\right)^{2} P_{-1, N}^{(2)}\right) q \\
D & :=q\left(-\frac{\gamma}{2} \partial_{y}^{-1} G(0)+a_{1} \partial_{y}+a_{6}\right) q^{-1}-\rho q^{-1}\left(\omega \cdot \partial_{\varphi} q\right)
\end{aligned}
$$

We choose the function $q$ so that the coefficients of the highest order terms of the off-diagonal operators $B$ and $C$ satisfy

$$
q^{-2} a_{2}^{2}=q^{2} a_{2}^{2} a_{3}=m_{\frac{3}{2}}(\varphi),
$$

with $m_{\frac{3}{2}}(\varphi)$ independent of $x$. This is achieved by choosing

$$
q:=\left(\frac{1}{a_{3}}\right)^{1 / 4}
$$

and, recalling (7.56), the function $\beta$, so that

$$
\left(1+\beta_{x}(\varphi, x)\right)^{3} c(\varphi, x)=m(\varphi),
$$

with $m(\varphi)$ independent of $x$ (the function $c$ is defined in (7.22)). The solution of (7.67) is

$$
m(\varphi):=\left(\frac{1}{2 \pi} \int_{\mathbb{T}} c(\varphi, x)^{-1 / 3} \mathrm{~d} x\right)^{-3}, \quad \beta(\varphi, x):=\partial_{x}^{-1}\left(\left(\frac{m(\varphi)}{c(\varphi, x)}\right)^{1 / 3}-1\right) .
$$

In such a way, by (7.56), we obtain (7.65) with $m_{\frac{3}{2}}(\varphi):=\sqrt{m(\varphi)}$. By (7.68) and (7.22) we have

$$
m_{\frac{3}{2}}:=m_{\frac{3}{2}}(\varphi)=\mathcal{P}^{-1}\left(\frac{1}{2 \pi} \int_{\mathbb{T}} \sqrt{1+\eta_{x}^{2}(\varphi, x)} \mathrm{d} x\right)^{-\frac{3}{2}} .
$$

Note that, since by (7.65) the function $q^{-1} a_{2}$ is independent of $x$, we have

$$
B=-q^{-1} a_{2} G(0) a_{2} q^{-1}=-q^{-2} a_{2}^{2} G(0) .
$$


Moreover we have the expansion

$$
\begin{aligned}
q a_{2} \partial_{y} a_{3} \partial_{y} a_{2} q & =q^{2} a_{2}^{2} a_{3} \partial_{y}^{2}+\left(q^{2} a_{2}^{2} a_{3}\right)_{y} \partial_{y}+q a_{2}\left(a_{3}\left(q a_{2}\right)_{y}\right)_{y} \\
& \stackrel{(7.65)}{=} m_{\frac{3}{2}}(\varphi) \partial_{y}^{2}+a_{7}, \quad a_{7}:=q a_{2}\left(a_{3}\left(q a_{2}\right)_{y}\right)_{y} .
\end{aligned}
$$

In conclusion, the operator $\mathcal{L}_{3}$ in (7.60) is, in view of (7.61)-(7.64) and (7.70), (7.71),

$$
\begin{aligned}
\mathcal{L}_{3}=\mathcal{Q}^{-1} \mathcal{L}_{2} \mathcal{Q}= & \omega \cdot \partial_{\varphi}+\frac{1}{\rho}\left(\begin{array}{cc}
-\frac{\gamma}{2} G(0) \partial_{y}^{-1} & -m_{\frac{3}{2}} G(0) \\
m_{\frac{3}{2}}\left(-\kappa \partial_{y}^{2}+g-\left(\frac{\gamma}{2}\right)^{2} \partial_{y}^{-1} G(0) \partial_{y}^{-1}\right)-\frac{\gamma}{2} \partial_{y}^{-1} G(0)
\end{array}\right) \\
& +\frac{1}{\rho}\left(\begin{array}{cc}
a_{1} \partial_{y}+a_{8} & 0 \\
a_{9}+P_{-1, N}^{(3)} & a_{1} \partial_{y}+a_{10}
\end{array}\right)+\mathbf{R}_{3}^{\Psi}+\mathbf{T}_{3, N},
\end{aligned}
$$

where

$$
\begin{aligned}
& a_{8}:=a_{1} q^{-1} q_{y}+\rho q^{-1}\left(\omega \cdot \partial_{\varphi} q\right)+a_{4}, \quad a_{9}:=a_{5} q^{2}+g\left(q^{2}-m_{\frac{3}{2}}\right)-\kappa a_{7}, \\
& a_{10}:=-a_{1} q^{-1} q_{y}-\rho q^{-1}\left(\omega \cdot \partial_{\varphi} q\right)+a_{6}, \\
& P_{-1, N}^{(3)}:=-\left(\frac{\gamma}{2}\right)^{2}\left(q P_{-1, N}^{(2)} q+\left(q^{2}-m_{\frac{3}{2}}\right) G(0) \partial_{y}^{-2}+q\left[G(0) \partial_{y}^{-2}, q-1\right]\right),
\end{aligned}
$$

with $P_{-1, N}^{(3)} \in \mathrm{OPS}^{-1}$ and where $\mathbf{R}_{3}^{\Psi} \in \mathrm{OP} S^{-\infty}, \mathbf{T}_{3, N}$ are the smoothing remainders

$$
\begin{aligned}
& \mathbf{R}_{3}^{\Psi}:=\frac{1}{\rho}\left(\begin{array}{cc}
-\frac{\gamma}{2} q^{-1}[\mathcal{H} T(\mathrm{~h}), q-1] & 0 \\
0 & -\frac{\gamma}{2} q\left[\mathcal{H} T(\mathrm{~h}), q^{-1}-1\right]
\end{array}\right)+\mathcal{Q}^{-1} \mathbf{R}_{2}^{\Psi} \mathcal{Q}, \\
& \mathbf{T}_{3, N}:=\mathcal{Q}^{-1} \mathbf{T}_{2, N} \mathcal{Q}
\end{aligned}
$$

Step 3: We now conjugate $\mathcal{L}_{3}$ in (7.72), where we rename the space variable $y$ by $x$, by the symplectic transformation (cfr. (3.42))

$$
\widetilde{\mathcal{M}}:=\left(\begin{array}{cc}
\Lambda & 0 \\
0 & \Lambda^{-1}
\end{array}\right), \quad \widetilde{\mathcal{M}}^{-1}:=\left(\begin{array}{cc}
\Lambda^{-1} & 0 \\
0 & \Lambda
\end{array}\right),
$$

where $\Lambda \in \mathrm{OPS}^{-\frac{1}{4}}$ is the Fourier multiplier

$$
\Lambda:=\frac{1}{\sqrt{g}} \pi_{0}+M(D), \quad \text { with inverse } \Lambda^{-1}:=\sqrt{g} \pi_{0}+M(D)^{-1} \in \mathrm{OP}^{\frac{1}{4}},
$$

with $\pi_{0}$ defined in (3.21) and $M(D)$ in (2.21). We have the identity

$$
\Lambda\left(-\kappa \partial_{x}^{2}+g-\left(\frac{\gamma}{2}\right)^{2} \partial_{x}^{-1} G(0) \partial_{x}^{-1}\right) \Lambda=\Lambda^{-1} G(0) \Lambda^{-1}+\pi_{0}=\omega(\kappa, D)+\pi_{0},
$$

where $\omega(\kappa, D)$ is defined in (2.23). In (7.79) and (7.80) we mean that the symbols of $M(D), M(D)^{-1}$ and $\omega(\kappa, D)$ are extended to 0 at $j=0$, multiplying them by the cut-off function $\chi$ defined in (3.10). Thus we obtain

$$
\mathcal{L}_{4}:=\widetilde{\mathcal{M}}^{-1} \mathcal{L}_{3} \widetilde{\mathcal{M}}=\omega \cdot \partial_{\varphi}+\frac{1}{\rho}\left(\begin{array}{cc}
-\frac{\gamma}{2} G(0) \partial_{x}^{-1} & -m_{\frac{3}{2}}(\varphi) \omega(\kappa, D) \\
m_{\frac{3}{2}}(\varphi) \omega(\kappa, D) & -\frac{\gamma}{2} G(0) \partial_{x}^{-1}
\end{array}\right)+\left(\begin{array}{cc}
0 & 0 \\
\pi_{0} & 0
\end{array}\right)
$$




$$
+\frac{1}{\rho}\left(\begin{array}{cc}
a_{1} \partial_{x}+P_{0}^{(41)} & 0 \\
P_{-\frac{1}{2}}^{(43)} & a_{1} \partial_{x}+P_{0}^{(44)}
\end{array}\right)+\mathbf{R}_{4}^{\Psi}+\mathbf{T}_{4, N},
$$

where

$$
\begin{aligned}
& P_{0}^{(41)}:=\Lambda^{-1}\left[a_{1} \partial_{x}, \Lambda\right]+\Lambda^{-1} a_{8} \Lambda \in \mathrm{OP} S^{0}, \\
& P_{-\frac{1}{2}, N}^{(43)}:=\Lambda a_{9} \Lambda+\Lambda P_{-1, N}^{(3)} \Lambda \in \mathrm{OP} S^{-\frac{1}{2}}, \\
& P_{0}^{(44)}:=\Lambda\left[a_{1} \partial_{x}, \Lambda^{-1}\right]+\Lambda a_{10} \Lambda^{-1} \in \mathrm{OP} S^{0},
\end{aligned}
$$

and $\mathbf{R}_{4}^{\Psi}, \mathbf{T}_{4, N}$ are the smoothing remainders

$$
\begin{aligned}
& \mathbf{R}_{4}^{\Psi}:=\left(\begin{array}{cr}
0 & 0 \\
\left(\rho^{-1} m_{\frac{3}{2}}-1\right) \pi_{0} & 0
\end{array}\right)+\widetilde{\mathcal{M}}^{-1} \mathbf{R}_{3}^{\Psi} \widetilde{\mathcal{M}} \in \mathrm{OPS}^{-\infty}, \\
& \mathbf{T}_{4, N}:=\widetilde{\mathcal{M}}^{-1} \mathbf{T}_{3, N} \widetilde{\mathcal{M}}=-\frac{\gamma^{2}}{4 \rho}\left(\begin{array}{cr}
0 & 0 \\
\Lambda q \mathrm{R}_{2, N} q \Lambda & 0
\end{array}\right) .
\end{aligned}
$$

Step 4: We finally move in complex coordinates, conjugating the operator $\mathcal{L}_{4}$ in (7.81) via the transformation $\mathcal{C}$ defined in (2.24). We use the transformation formula (3.15). We choose the function $p(\varphi)$ in (7.17) in order to obtain a constant coefficient at the highest order. More precisely we choose the periodic function $p(\varphi)$ such that

$$
\frac{m_{\frac{3}{2}}}{\rho} \stackrel{(7.69),(7.20)}{=} \mathcal{P}^{-1}\left(\frac{\left(\frac{1}{2 \pi} \int_{\mathbb{T}} \sqrt{1+\eta_{x}^{2}(\varphi, x)} \mathrm{d} x\right)^{-\frac{3}{2}}}{1+\omega \cdot \partial_{\varphi} p}\right)=m_{\frac{3}{2}}
$$

is a real constant independent of $\varphi$. Thus, recalling (3.9), we define the periodic function

$$
p(\varphi):=\left(\omega \cdot \partial_{\varphi}\right)_{\operatorname{ext}}^{-1}\left(\frac{1}{\mathrm{~m}_{\frac{3}{2}}}\left(\frac{1}{2 \pi} \int_{\mathbb{T}} \sqrt{1+\eta_{x}^{2}(\varphi, x)} \mathrm{d} x\right)^{-\frac{3}{2}}-1\right)
$$

and the real constant

$$
\mathrm{m}_{\frac{3}{2}}:=\frac{1}{(2 \pi)^{v}} \int_{\mathbb{T}^{v}}\left(\frac{1}{2 \pi} \int_{\mathbb{T}} \sqrt{1+\eta_{x}^{2}(\varphi, x)} \mathrm{d} x\right)^{-\frac{3}{2}} \mathrm{~d} \varphi .
$$

Note that (7.86) holds for $\omega \in \mathrm{DC}(v, \tau)$. Moreover, by Lemmata 3.2, 7.3 and (7.16), $p$ satisfies (7.19) and it is odd in $\varphi$. Let

$$
\Pi_{0}:=-\mathrm{i} \mathcal{C}^{-1}\left(\begin{array}{cc}
0 & 0 \\
\pi_{0} & 0
\end{array}\right) \mathcal{C}=\frac{1}{2}\left(\begin{array}{cc}
\pi_{0} & \pi_{0} \\
-\pi_{0} & -\pi_{0}
\end{array}\right) .
$$

Lemma 7.9. Let $N \in \mathbb{N}$, $q_{0} \in \mathbb{N}_{0}$. For all $\omega \in \mathrm{DC}(v, \tau)$, we have that

$$
\begin{aligned}
\mathcal{L}_{5} & :=(\mathcal{E} \mathcal{Q} \widetilde{\mathcal{M}} \mathcal{C})^{-1} \mathcal{L}_{1}(\mathcal{E} \mathcal{Q} \widetilde{\mathcal{M}} \mathcal{C}) \\
& =\omega \cdot \partial_{\varphi}+\operatorname{im}_{\frac{3}{2}} \boldsymbol{\Omega}(\kappa, D)+\mathbf{A}_{1} \partial_{x}+\mathrm{i} \Pi_{0}+\mathbf{R}_{5}^{(0, d)}+\mathbf{R}_{5}^{(0, o)}+\mathbf{T}_{5, N},
\end{aligned}
$$

where: 
1. The operators $\mathcal{E}^{ \pm 1}$ are $\mathcal{D}^{k_{0}}-\left(k_{0}+1\right)$-tame, the operators $\mathcal{E}^{ \pm 1}-\mathrm{Id},\left(\mathcal{E}^{ \pm 1}-\mathrm{Id}\right)^{*}$ are $\mathcal{D}^{k_{0}}-\left(k_{0}+2\right)$-tame and the operators $\mathcal{Q}^{ \pm 1}, \mathcal{Q}^{ \pm 1}-\mathrm{Id},\left(\mathcal{Q}^{ \pm 1}-\mathrm{Id}\right)^{*}$ are $\mathcal{D}^{k_{0}}$-tame with tame constants satisfying, for some $\sigma:=\sigma\left(\tau, v, k_{0}\right)>0$ and for all $s_{0} \leqq s \leqq S$,

$$
\begin{aligned}
& \mathfrak{M}_{\mathcal{E}^{ \pm 1}}(s) \lesssim_{S} 1+\left\|\mathfrak{I}_{0}\right\|_{s+\sigma}^{k_{0}, v}, \quad \mathfrak{M}_{\mathcal{Q}^{ \pm 1}}(s) \lesssim_{S} 1+\left\|\mathfrak{I}_{0}\right\|_{s+\sigma}^{k_{0}, v} \\
& \mathfrak{M}_{\mathcal{E}^{ \pm 1}-\mathrm{Id}}(s)+\mathfrak{M}_{\left(\mathcal{E}^{ \pm 1}-\mathrm{Id}\right)^{*}}(s) \lesssim_{S} \varepsilon^{2}\left(1+\left\|\mathfrak{I}_{0}\right\|_{s+\sigma}^{k_{0}, v}\right) \\
& \mathfrak{M}_{\mathcal{Q}^{ \pm 1}-\mathrm{Id}}(s)+\mathfrak{M}_{\left(\mathcal{Q}^{ \pm 1}-\mathrm{Id}\right)^{*}}(s) \lesssim_{S} \varepsilon^{2}\left(1+\left\|\mathfrak{I}_{0}\right\|_{s+\sigma}^{k_{0}, v}\right)
\end{aligned}
$$

2. the constant $\mathrm{m}_{\frac{3}{2}} \in \mathbb{R}$ defined in (7.88) satisfies $\left|\mathrm{m}_{\frac{3}{2}}-1\right|^{k_{0}, v} \lesssim \varepsilon^{2}$;

3. $\boldsymbol{\Omega}(\kappa, D)$ is the Fourier multiplier (see (2.25), (2.26))

$$
\boldsymbol{\Omega}(\kappa, D)=\left(\begin{array}{cc}
\Omega(\kappa, D) & 0 \\
0 & -\overline{\Omega(\kappa, D)}
\end{array}\right), \quad \Omega(\kappa, D)=\omega(\kappa, D)+\mathrm{i} \frac{\gamma}{2} \partial_{x}^{-1} G(0) ;
$$

4. the matrix of functions $\mathbf{A}_{1}$ is

$$
\mathbf{A}_{1}:=\left(\begin{array}{cc}
a_{1}^{(d)} & 0 \\
0 & a_{1}^{(d)}
\end{array}\right)
$$

for a real function $a_{1}^{(d)}(\varphi, x)$ which is a quasi-periodic traveling wave, even $(\varphi, x)$, satisfying, for some $\sigma:=\sigma\left(k_{0}, \tau, v\right)>0$ and for all $s \geqq s_{0}$,

$$
\left\|a_{1}^{(d)}\right\|_{s}^{k_{0}, v} \lesssim_{s} \varepsilon\left(1+\left\|\Im_{0}\right\|_{s+\sigma}^{k_{0}, v}\right)
$$

5. $\mathbf{R}_{5}^{(0, d)}$ and $\mathbf{R}_{5}^{(0, o)}$ are pseudodifferential operators in $\mathrm{OP} S^{0}$ of the form

$$
\begin{aligned}
& \mathbf{R}_{5}^{(0, d)}:=\left(\begin{array}{cc}
r_{5}^{(d)}(\varphi, x, D) & 0 \\
0 & r_{5}^{(d)}(\varphi, x, D)
\end{array}\right) \in \mathrm{OPS}^{0} \\
& \mathbf{R}_{5}^{(0, o)}:=\left(\begin{array}{cc}
\frac{0}{r_{5}^{(d)}(\varphi, x, D)} & 0
\end{array}\right) \in \mathrm{OPS}^{(o)}(\varphi, x, D)
\end{aligned}
$$

reversibility and momentum preserving, satisfying, for some $\sigma_{N}:=\sigma(\tau, \nu, N)>$ 0 , for all $s \geqq s_{0}, \alpha \in \mathbb{N}_{0}$,

$$
\left\|\mathbf{R}_{5}^{(0, d)}\right\|_{0, s, \alpha}^{k_{0}, v}+\left\|\mathbf{R}_{5}^{(0, o)}\right\|_{0, s, \alpha}^{k_{0}, v} \lesssim_{s, N, \alpha} \varepsilon\left(1+\left\|\Im_{0}\right\|_{s+\sigma_{N}+2 \alpha}^{k_{0}, v}\right)
$$

6. For any $q \in \mathbb{N}_{0}^{v}$ with $|\mathrm{q}| \leqq \mathrm{q}_{0}, n_{1}, n_{2} \in \mathbb{N}_{0}$ with $n_{1}+n_{2} \leqq N-\left(k_{0}+\mathrm{q}_{0}\right)+\frac{5}{2}$, the operator $\langle D\rangle^{n_{1}} \partial_{\varphi}^{\mathrm{q}} \mathbf{T}_{5, N}(\varphi)\langle D\rangle^{n_{2}}$ is $\mathcal{D}^{k_{0}}$-tame with a tame constant satisfying, for some $\sigma_{N}\left(\mathrm{q}_{0}\right):=\sigma_{N}\left(\mathrm{q}_{0}, k_{0}, \tau, v\right)>0$ and for any $s_{0} \leqq s \leqq S$,

$$
\mathfrak{M}_{\langle D\rangle^{n}{ }_{1} \partial_{\varphi}^{\mathrm{q}} \mathbf{T}_{5, N}(\varphi)\langle D\rangle^{n_{2}}}(s) \lesssim_{S, N, \mathrm{q}_{0}} \varepsilon\left(1+\left\|\mathfrak{I}_{0}\right\|_{S+\sigma_{N}\left(\mathrm{q}_{0}\right)}^{k_{0}, v}\right)
$$


7. Moreover, for any $s_{1}$ as in (7.15), $\alpha \in \mathbb{N}_{0}$, $q \in \mathbb{N}_{0}^{v}$, with $|\mathrm{q}| \leqq q_{0}$, and $n_{1}, n_{2} \in \mathbb{N}_{0}$, with $n_{1}+n_{2} \leqq N-q_{0}+\frac{3}{2}$,

$$
\begin{aligned}
& \left\|\Delta_{12}(\mathcal{A}) h\right\|_{s_{1}} \lesssim_{s_{1}} \varepsilon\left\|i_{1}-i_{2}\right\|_{s_{1}+\sigma}\|h\|_{s_{1}+\sigma}, \mathcal{A} \in\left\{\mathcal{E}^{ \pm 1},\left(\mathcal{E}^{ \pm 1}\right)^{*}, \mathcal{Q}^{ \pm 1}=\left(\mathcal{Q}^{ \pm 1}\right)^{*}\right\} \\
& \left\|\Delta_{12} a_{1}^{(d)}\right\|_{s_{1}} \lesssim_{s_{1}} \varepsilon\left\|i_{1}-i_{2}\right\|_{s_{1}+\sigma},\left|\Delta_{12 \mathrm{~m}_{3}}\right| \lesssim_{2}\left\|\varepsilon^{2}\right\| i_{1}-i_{2} \|_{s_{1}+\sigma} \\
& \left\|\Delta_{12} \mathbf{R}_{5}^{(d)}\right\|_{0, s_{1}, \alpha}+\left\|\Delta_{12} \mathbf{R}_{5}^{(o)}\right\|_{0, s_{1}, \alpha} \lesssim_{s_{1}, N, \alpha} \varepsilon\left\|i_{1}-i_{2}\right\|_{s_{1}+\sigma_{N}+2 \alpha} \\
& \left\|\langle D\rangle^{n_{1}} \partial_{\varphi}^{\mathrm{q}} \mathbf{T}_{5, N}(\varphi)\langle D\rangle^{n_{2}}\right\|_{\mathcal{L}\left(H^{\left.s_{1}\right)}\right.} \lesssim_{s_{1}, N, \mathrm{q}_{0}} \varepsilon\left\|i_{1}-i_{2}\right\|_{s_{1}+\sigma_{N}\left(\mathrm{q}_{0}\right)} \cdot
\end{aligned}
$$

The real operator $\mathcal{L}_{5}$ is Hamiltonian, reversible and momentum preserving.

Proof. By the expression of $\mathcal{L}_{4}$ in (7.81), using (3.15), and (7.86), we obtain that $\mathcal{L}_{5}$ has the form (7.89). The functions $\beta$ and $q$, defined respectively in (7.68) and (7.66) with $a_{3}$ defined in (7.56), satisfy, by Lemmata 3.8, 3.2 and (7.28), for some $\sigma:=\sigma\left(k_{0}, \tau, v\right)>0$ and for all $s \geqq s_{0}$,

$$
\|\beta\|_{s}^{k_{0}, v} \lesssim_{s} \varepsilon^{2}\left(1+\left\|\Im_{0}\right\|_{s+\sigma}^{k_{0}, v}\right), \quad\left\|q^{ \pm 1}-1\right\|_{s}^{k_{0}, v} \lesssim_{s} \varepsilon^{2}\left(1+\left\|\Im_{0}\right\|_{s+\sigma}^{k_{0}, v}\right) .
$$

The estimates (7.90)-(7.92) follow by Lemmata 3.12, 3.13, 3.8, (7.103) and writing

$$
(\mathcal{B}-\mathrm{Id}) h=\beta \mathcal{B}_{\tau}\left[h_{x}\right], \quad \mathcal{B}_{\tau}[h](\varphi, x):=\int_{0}^{1} h_{x}(\varphi, x+\tau \beta(\varphi, x)) \mathrm{d} \tau,
$$

$\mathcal{B}^{*} h(\varphi, y)=(1+\breve{\beta}(\varphi, y)) h(\varphi, y+\breve{\beta}(\varphi, y))$, and similar expressions for $\mathcal{B}^{-1}-\mathrm{Id}$, $\left(\mathcal{B}^{-1}\right)^{*}$. The estimate for $\mathrm{m}_{\frac{3}{2}}$ follows by (7.88), Lemma 3.2 and (7.16). The real function $a_{1}^{(d)}$ in (7.94) is

$$
a_{1}^{(d)}(\varphi, x):=\rho(\varphi)^{-1} a_{1}(\varphi, x),
$$

where $\rho$ and $a_{1}$ are defined respectively in (7.20) and (7.55). Recalling Lemmata 7.3 and 7.6, the function $a_{1}^{(d)}$ is a quasi-periodic traveling wave, even in $(\varphi, x)$. Moreover, (7.95) follows by Lemma 3.2 and (7.16), (7.19), (7.28), (7.103). By direct computations, we have

$$
\begin{aligned}
r_{5}^{(d)}(\varphi, x, D) & :=\frac{1}{2 \rho}\left(P_{0}^{(41)}+P_{0}^{(44)}+\mathrm{i} P_{-\frac{1}{2}, N}^{(43)}+\gamma\left(\rho \mathrm{m}_{\frac{3}{2}}-1\right) G(0) \partial_{x}^{-1}\right), \\
r_{5}^{(o)}(\varphi, x, D) & :=\frac{1}{2 \rho}\left(P_{0}^{(41)}-P_{0}^{(44)}+\mathrm{i} P_{-\frac{1}{2}, N}^{(43)}\right),
\end{aligned}
$$

where $P_{0}^{(41)}, P_{-\frac{1}{2}, N}^{(43)}, P_{0}^{(44)}$ are defined in (7.82), (7.83), (7.84) and $\rho \mathrm{m}_{\frac{3}{2}}=m_{\frac{3}{2}}(\varphi)$ with $m_{\frac{3}{2}}(\varphi)$ defined in (7.69) (cfr. (7.86)). Therefore, the estimate (7.97) follows by (7.73), (7.71), (7.55), (7.56), (7.57), (7.58), (7.75), (7.52), (7.79), (2.21), applying Lemmata 3.5, 3.6, 3.8, 3.2, Proposition 3.9 and estimates (7.16), (7.19), (7.28), (7.103). The estimate (7.98), where

$$
\mathbf{T}_{5, N}:=\mathcal{C}^{-1}\left(\mathbf{R}_{4}^{\Psi}+\mathbf{T}_{4, N}\right) \mathcal{C},
$$


follows by (7.85), (7.77), (7.76), (7.59), (7.53), (7.50), (7.48), (7.46), (7.43), Lemmata 3.12, 3.13, estimates (7.44), (7.49), Proposition 3.9 and (7.90), (7.103), Lemma 3.10 and Lemmata 2.34, 2.32 in [6]. The estimates (7.99), (7.100), (7.101), (7.102) are proved in the same fashion. Since the transformations $\mathcal{E}, \mathcal{Q}, \widetilde{\mathcal{M}}$ are symplectic, the operator $\mathcal{L}_{4}$ is Hamiltonian. Hence the operator $\mathcal{L}_{5}$ obtained conjugating with $\mathcal{C}$ is Hamiltonian according to (3.41). By Lemma 7.3, the functions $\beta(\varphi, x)$ and $q(\varphi, x)$, defined in (7.68), (7.66) (with $a_{3}$ defined in (7.56)), are both quasi-periodic traveling waves, respectively $\operatorname{odd}(\varphi, x)$ and even $(\varphi, x)$. Therefore, the transformations $\mathcal{E}$ and $\mathcal{Q}$ are momentum and reversibility preserving. Moreover, also $\widetilde{\mathcal{M}}$ and $\mathcal{C}$ are momentum and reversibility preserving (writing the involution in complex variables as in (2.29)). Hence, since $\mathcal{L}_{1}$ is momentum preserving and reversible (Lemma 7.8), the operator $\mathcal{L}_{5}$ is momentum preserving and reversible as well, in particular the operators $\mathbf{R}_{5}^{(0, d)}$ and $\mathbf{R}_{5}^{(0, o)}$ in (7.96) (for example check the definition in (7.105), see also Remark 3.20).

\subsection{Symmetrization up to Smoothing Remainders}

The goal of this section is to transform the operator $\mathcal{L}_{5}$ in (7.89) into the operator $\mathcal{L}_{6}$ in (7.108) which is block diagonal up to a regularizing remainder. From this step we do not preserve any further the Hamiltonian structure, but only the reversible and momentum preserving one (it is now sufficient for proving Theorem 5.2).

Lemma 7.10. Fix $M, N \in \mathbb{N}$, $q_{0} \in \mathbb{N}_{0}$. There exist real, reversibility and momentum preserving operator matrices $\left\{\mathbf{X}_{m}\right\}_{m=1}^{M}$ of the form

$$
\mathbf{X}_{m}:=\left(\begin{array}{cc}
0 & \chi_{m}(\varphi, x, D) \\
\chi_{m}(\varphi, x, D) & 0
\end{array}\right), \quad \chi_{m}(\varphi, x, \xi) \in S^{-\frac{1}{2}-m},
$$

such that, conjugating the operator $\mathcal{L}_{5}$ in (7.89) via the map

$$
\boldsymbol{\Phi}_{M}:=e^{\mathbf{X}_{1}} \circ \cdots \circ e^{\mathbf{X}_{M}},
$$

we obtain the real, reversible and momentum preserving operator

$$
\begin{aligned}
\mathcal{L}_{6} & :=\mathcal{L}_{6}^{(M)}:=\boldsymbol{\Phi}_{M}^{-1} \mathcal{L}_{5} \boldsymbol{\Phi}_{M} \\
& =\omega \cdot \partial_{\varphi}+\mathrm{im}_{\frac{3}{2}} \boldsymbol{\Omega}(\kappa, D)+\mathbf{A}_{1} \partial_{x}+\mathrm{i} \Pi_{0}+\mathbf{R}_{6}^{(0, d)}+\mathbf{R}_{6}^{(-M, o)}+\mathbf{T}_{6, N},
\end{aligned}
$$

with a block-diagonal operator

$$
\mathbf{R}_{6}^{(0, d)}:=\mathbf{R}_{6, M}^{(0, d)}:=\left(\begin{array}{cc}
r_{6}^{(d)}(\varphi, x, D) & 0 \\
0 & r_{6}^{(d)}(\varphi, x, D)
\end{array}\right) \in \mathrm{OPS}^{0},
$$

and a smoothing off diagonal remainder

$$
\mathbf{R}_{6}^{(-M, o)}:=\mathbf{R}_{6, M}^{(-M, o)}:=\left(\begin{array}{c}
0 \\
r_{6}^{(o)}(\varphi, x, D) \\
r_{6}^{(o)}(\varphi, x, D) \\
0
\end{array}\right) \in \mathrm{OPS}^{-M}
$$


both reversibility and momentum preserving, which satisfy for all $\alpha \in \mathbb{N}_{0}$, for some $\sigma_{N}:=\sigma_{N}\left(k_{0}, \tau, \nu, N\right)>0, \aleph_{M}(\alpha)>0$, for all $s \geqq s_{0}$,

$$
\left\|\mathbf{R}_{6}^{(0, d)}\right\|_{0, s, \alpha}^{k_{0}, v}+\left\|\mathbf{R}_{6}^{(-M, o)}\right\|_{-M, s, \alpha}^{k_{0}, v} \lesssim s, M, N, \alpha \varepsilon\left(1+\left\|\Im_{0}\right\|_{s+\sigma_{N}+\aleph_{M}(\alpha)}^{k_{0}, v}\right) .
$$

For any $\mathrm{q} \in \mathbb{N}_{0}^{v}$ with $|\mathrm{q}| \leqq \mathrm{q}_{0}, n_{1}, n_{2} \in \mathbb{N}_{0}$ with $n_{1}+n_{2} \leqq N-\left(k_{0}+\mathrm{q}_{0}\right)+\frac{5}{2}$, the operator $\langle D\rangle^{n_{1}} \partial_{\varphi}^{q} \mathbf{T}_{6, N}(\varphi)\langle D\rangle^{n_{2}}$ is $\mathcal{D}^{k_{0}}$-tame with a tame constant satisfying, for some $\sigma_{N}\left(q_{0}\right):=\sigma_{N}\left(k_{0}, \tau, v, q_{0}\right)$, for any $s_{0} \leqq s \leqq S$,

$$
\mathfrak{M}_{\langle D\rangle^{n_{1}} \partial_{\varphi}^{\mathrm{q}} \mathbf{T}_{6, N}(\varphi)\langle D\rangle^{n_{2}}}(s) \lesssim S, M, N, \mathrm{q}_{0} \varepsilon\left(1+\left\|\mathfrak{I}_{0}\right\|_{s+\sigma_{N}\left(\mathrm{q}_{0}\right)+\aleph_{M}(0)}^{k_{0}, v}\right)
$$

The conjugation map $\boldsymbol{\Phi}_{M}$ in (7.107) satisfies, for all $s \geqq s_{0}$,

$$
\left\|\boldsymbol{\Phi}_{M}^{ \pm 1}-\mathrm{Id}\right\|_{0, s, 0}^{k_{0}, v}+\left\|\left(\boldsymbol{\Phi}_{M}^{ \pm 1}-\mathrm{Id}\right)^{*}\right\|_{0, s, 0}^{k_{0}, v} \lesssim_{s, M, N} \varepsilon\left(1+\left\|\Im_{0}\right\|_{s+\sigma_{N}+\aleph_{M}(0)}^{k_{0},}\right) .
$$

Furthermore, for any $s_{1}$ as in (7.15), $\alpha \in \mathbb{N}_{0}$, $q \in \mathbb{N}_{0}^{v}$, with $|\mathrm{q}| \leqq \mathrm{q}_{0}$, and $n_{1}, n_{2} \in \mathbb{N}_{0}$, with $n_{1}+n_{2} \leqq N-q_{0}+\frac{3}{2}$, we have

$$
\begin{aligned}
& \left\|\Delta_{12} \mathbf{R}_{6}^{(0, d)}\right\|_{0, s_{1}, \alpha}+\left\|\Delta_{12} \mathbf{R}_{6}^{(-M, o)}\right\|_{-s_{1}, M, \alpha} \lesssim_{s_{1}, M, N, \alpha} \varepsilon\left\|i_{1}-i_{2}\right\|_{s_{1}+\sigma_{N}+\aleph_{M}(\alpha)} \\
& \left\|\langle D\rangle^{n_{1}} \partial_{\varphi}^{\mathrm{q}} \Delta_{12} \mathbf{T}_{6, N}\langle D\rangle^{n_{2}}\right\|_{\mathcal{L}\left(H^{\left.s_{1}\right)}\right.} \lesssim_{s_{1}, M, N, \mathrm{q}_{0}} \varepsilon\left\|i_{1}-i_{2}\right\|_{s_{1}+\sigma_{N}\left(\mathrm{q}_{0}\right)+\aleph_{M}(0)} \\
& \left\|\Delta_{12} \boldsymbol{\Phi}_{M}^{ \pm 1}\right\|_{0, s_{1}, 0}+\left\|\Delta_{12}\left(\boldsymbol{\Phi}_{M}^{ \pm 1}\right)^{*}\right\|_{0, s_{1}, 0} \lesssim_{s_{1}, M, N} \varepsilon\left\|i_{1}-i_{2}\right\|_{s_{1}+\sigma_{N}+\aleph_{M}(0)}
\end{aligned}
$$

Proof. The proof is inductive on the index $M$. The operator $\mathcal{L}_{6}^{(0)}:=\mathcal{L}_{5}$ satisfy (7.110)-(7.111) with $\aleph_{0}(\alpha):=2 \alpha$, by Lemma 7.9. Suppose we have done already $M$ steps obtaining an operator $\mathcal{L}_{6}^{(M)}$ as in (7.108) with a remainder $\Phi_{M}^{-1} \mathbf{T}_{5, N} \boldsymbol{\Phi}_{M}$, instead of $\mathbf{T}_{6, N}$. We now show how to perform the $(M+1)$-th step. Define the symbol

$$
\chi_{M+1}(\varphi, x, \xi):=-\left(2 \operatorname{im}_{\frac{3}{2}} \omega(\kappa, \xi)\right)^{-1} r_{6, M}^{(o)}(\varphi, x, \xi) \chi(\xi) \in S^{-\frac{3}{2}-M},
$$

where $\chi$ is the cut-off function defined in (3.10) and $\omega(\kappa, \xi)$ is the symbol (cfr. (2.23))

$$
\begin{aligned}
& \omega(\kappa, \xi):=\sqrt{G(0 ; \xi)\left(\kappa \xi^{2}+g+\frac{\gamma^{2}}{4} \frac{G(0 ; \xi)}{\xi^{2}}\right)} \in S^{\frac{3}{2}}, \\
& G(0 ; \xi):= \begin{cases}\chi(\xi)|\xi| \tanh (\mathrm{h}|\xi|), & \mathrm{h}<+\infty \\
\chi(\xi)|\xi|, & \mathrm{h}=+\infty .\end{cases}
\end{aligned}
$$

Note that $\chi_{M+1}$ in (7.116) is well defined because $\omega(\kappa, \xi)$ is positive on the support of $\chi(\xi)$. We conjugate the operator $\mathcal{L}_{6}^{(M)}$ in (7.108) by the flow generated by $\mathbf{X}_{M+1}$ 
of the form (7.106) with $\chi_{M+1}(\varphi, x, \xi)$ defined in (7.116). By (7.110) and Lemma 7.9-2, for any $s \geqq s_{0}$ and $\alpha \in \mathbb{N}_{0}$,

$$
\left\|\mathbf{X}_{M+1}\right\|_{-\frac{1}{2}-(M+1), s, \alpha}^{k_{0}, v} \lesssim s, M, \alpha \varepsilon\left(1+\left\|\Im_{0}\right\|_{s+\sigma_{N}+\aleph_{M}(\alpha)}^{k_{0}, v}\right) .
$$

Therefore, by Lemmata 3.7, 3.5 and the induction assumption (7.112) for $\boldsymbol{\Phi}_{M}$, the conjugation map $\boldsymbol{\Phi}_{M+1}:=\boldsymbol{\Phi}_{M} e^{\mathbf{X}_{M+1}}$ is well defined and satisfies estimate (7.112) with $M+1$. By the Lie expansion (3.16) we have

$$
\begin{aligned}
& \mathcal{L}_{6}^{(M+1)}:=e^{-\mathbf{X}_{M+1}} \mathcal{L}_{6}^{(M)} e^{\mathbf{X}_{M+1}} \\
& =\omega \cdot \partial_{\varphi}+\mathrm{im}_{\frac{3}{2}} \boldsymbol{\Omega}(\kappa, D)+\mathbf{A}_{1} \partial_{x}+\mathrm{i} \boldsymbol{\Pi}_{0}+\mathbf{R}_{6, M}^{(0, d)} \\
& -\left[\mathbf{X}_{M+1}, \mathrm{im}_{\frac{3}{2}} \boldsymbol{\Omega}(\kappa, D)\right]+\mathbf{R}_{6, M}^{(-M, o)}+\boldsymbol{\Phi}_{M+1}^{-1} \mathbf{T}_{5, N} \Phi_{M+1} \\
& -\int_{0}^{1} e^{-\tau \mathbf{X}_{M+1}}\left[\mathbf{X}_{M+1}, \omega \cdot \partial_{\varphi}+\mathbf{A}_{1}^{(d)} \partial_{x}+\mathrm{i} \Pi_{0}+\mathbf{R}_{6, M}^{(0, d)}\right] e^{\tau \mathbf{X}_{M+(7 \mathrm{~d} \imath 19)}} \\
& -\int_{0}^{1} e^{-\tau \mathbf{X}_{M+1}}\left[\mathbf{X}_{M+1}, \mathbf{R}_{6, M}^{(-M, o)}\right] e^{\tau \mathbf{X}_{M+1}} \mathrm{~d} \tau \\
& +\int_{0}^{1}(1-\tau) e^{-\tau \mathbf{X}_{M+1}}\left[\mathbf{X}_{M+1},\left[\mathbf{X}_{M+1}, \mathrm{im}_{\frac{3}{2}} \boldsymbol{\Omega}(\kappa, D)\right]\right] e^{\tau \mathbf{X}_{M+1}(\boldsymbol{d} \tau 121)}
\end{aligned}
$$

In view of (7.106), (7.93) and (7.109), we have that

$$
-\left[\mathbf{X}_{M+1}, \operatorname{im}_{\frac{3}{2}} \boldsymbol{\Omega}(\kappa, D)\right]+\mathbf{R}_{6, M}^{(-M, o)}=\left(\begin{array}{cc}
0 & Z_{M+1} \\
Z_{M+1} & 0
\end{array}\right)=: \mathbf{Z}_{M+1},
$$

where, denoting for brevity $\chi_{M+1}:=\chi_{M+1}(\varphi, x, \xi)$, it results

$$
\begin{aligned}
Z_{M+1}= & \operatorname{im}_{\frac{3}{2}}\left(\operatorname{Op}\left(\chi_{M+1}\right) \omega(\kappa, D)+\omega(\kappa, D) \mathrm{Op}\left(\chi_{M+1}\right)\right) \\
& +\mathrm{m}_{\frac{3}{2}} \frac{\gamma}{2}\left[\chi_{M+1}, \partial_{x}^{-1} G(0)\right]+\mathrm{Op}\left(r_{6, M}^{(o)}\right) .
\end{aligned}
$$

By (3.23), Lemma 3.5 and since $\chi_{M+1}(\varphi, x, \xi) \in S^{-\frac{3}{2}-M}$ by (7.116), we have that $\mathrm{Op}\left(\chi_{M+1}\right) \omega(\kappa, D)+\omega(\kappa, D) \mathrm{Op}\left(\chi_{M+1}\right)=\mathrm{Op}\left(2 \omega(\kappa, \xi) \chi_{M+1}(\varphi, x, \xi)\right)+r_{M+1}$, where $r_{M+1}$ is in $\mathrm{OP} S^{-M-1}$. By (7.116) and (7.122) $Z_{M+1}=\operatorname{im}_{\frac{3}{2}} r_{M+1}+\operatorname{m}_{\frac{3}{2}} \frac{\gamma}{2}\left[\chi_{M+1}, \partial_{x}^{-1} G(0)\right]+\mathrm{Op}\left(r_{6, M}^{(o)}(1-\chi(\xi))\right) \in \mathrm{OPS}^{-M-1}$.

The remaining pseudodifferential operators in (7.119)-(7.121) have order OP $S^{-M-\frac{3}{2}}$. Therefore the operator $\mathcal{L}_{6}^{(M+1)}$ in (7.118) has the form (7.108) at $M+1$ with

$$
\mathbf{R}_{6, M+1}^{(0, d)}+\mathbf{R}_{6, M+1}^{(-(M+1), o)}:=\mathbf{R}_{6, M}^{(0, d)}+\mathbf{Z}_{M+1}+(7.119)+(7.120)+(7.121)
$$

and a remainder $\boldsymbol{\Phi}_{M+1}^{-1} \mathbf{T}_{5, N} \boldsymbol{\Phi}_{M+1}$. By Lemmata 3.5, 3.6, the induction assumption (7.110), (7.117), (7.95), we conclude that $\mathbf{R}_{6, M+1}^{(0, d)}$ and $\mathbf{R}_{6, M+1}^{(-(M+1), o)}$ satisfy (7.110) 
at order $M+1$ for suitable constants $\aleph_{M+1}(\alpha)>\aleph_{M}(\alpha)$. Moreover the operator $\boldsymbol{\Phi}_{M+1}^{-1} \mathbf{T}_{5, N} \boldsymbol{\Phi}_{M+1}$ satisfies (7.112) (with $M+1$ ) by Lemmata 3.12, 3.13 and estimates (7.98), (7.112). Estimates (7.113), (7.114), (7.115) follow similarly. By (7.116), (3.43), Lemmata 3.19, 3.29, and the induction assumption that $\mathbf{R}_{6, M}^{(-M, o)}$ is reversible and momentum preserving, we conclude that $\mathbf{X}_{M+1}$ is reversibility and momentum preserving, and so are $e^{ \pm \mathbf{X}_{M+1}}$. By the induction assumption $\mathcal{L}_{6}^{(M)}$ is reversible and momentum preserving, and so $\mathcal{L}_{6}^{(M+1)}$ is reversible and momentum preserving as well, in particular the terms $\mathbf{R}_{6, M+1}^{(0, d)}+\mathbf{R}_{6, M+1}^{(-(M+1), o)}$ in (7.123).

Remark 7.11. The number of regularizing iterations $M \in \mathbb{N}$ will be fixed by the KAM reduction scheme in Section 8, see (8.5). Note that it is independent of the Sobolev index $s$.

So far the operator $\mathcal{L}_{6}$ of Lemma 7.10 depends on two indexes $M, N$ which provide respectively the order of the regularizing off-diagonal remainder $\mathbf{R}_{6}^{\left(-\frac{M}{2}, o\right)}$ and of the smoothing tame operator $\mathbf{T}_{6, N}$. From now on we fix

$$
N=M \text {. }
$$

\subsection{Reduction of the Order 1}

The goal of this section is to transform the operator $\mathcal{L}_{6}$ in (7.108), with $N=M$ (cfr. (7.124)), into the operator $\mathcal{L}_{8}$ in (7.146) whose coefficient in front of $\partial_{x}$ is a constant. We first eliminate the $x$-dependence and then the $\varphi$-dependence.

Space reduction. First we rewrite the operator $\mathcal{L}_{6}$ in (7.108), with $N=M$, as

$$
\mathcal{L}_{6}=\omega \cdot \partial_{\varphi}+\left(\begin{array}{cc}
P_{6} & 0 \\
0 & \frac{P_{6}}{2}
\end{array}\right)+\mathrm{i} \boldsymbol{\Pi}_{0}+\mathbf{R}_{6}^{(-M, o)}+\mathbf{T}_{6, M},
$$

having denoted

$$
P_{6}:=P_{6}(\varphi, x, D):=\operatorname{im}_{\frac{3}{2}} \Omega(\kappa, D)+a_{1}^{(d)}(\varphi, x) \partial_{x}+r_{6}^{(d)}(\varphi, x, D) .
$$

We conjugate $\mathcal{L}_{6}$ through the real operator

$$
\boldsymbol{\Phi}(\varphi):=\left(\begin{array}{cc}
\Phi(\varphi) & 0 \\
0 & \Phi(\varphi)
\end{array}\right)
$$

where $\Phi(\varphi):=\left.\Phi^{\tau}(\varphi)\right|_{\tau=1}$ is the time 1-flow of the PDE

$$
\left\{\begin{array}{l}
\partial_{\tau} \Phi^{\tau}(\varphi)=\mathrm{i} A(\varphi) \Phi^{\tau}(\varphi), \quad A(\varphi):=b(\varphi, x)|D|^{\frac{1}{2}}, \\
\Phi^{0}(\varphi)=\mathrm{Id},
\end{array}\right.
$$

and $b(\varphi, x)$ is a real, smooth, odd $(\varphi, x)$, periodic function chosen later, see (7.133), (7.135), (7.141). Usual energy estimates imply that the flow $\Phi^{\tau}(\varphi)$ of (7.127) is a bounded operator in $H_{x}^{s}$. The operator $\partial_{\lambda}^{k} \partial_{\varphi}^{\beta} \Phi$ loses $|D|^{\frac{|\beta|+|k|}{2}}$ derivatives, which are compensated by $\langle D\rangle^{-m_{1}}$ on the left hand side and $\langle D\rangle^{-m_{2}}$ on the right hand 
side, with $m_{1}, m_{2} \in \mathbb{R}$ satisfying $m_{1}+m_{2}=\frac{1}{2}(|\beta|+|k|)$, according to the tame estimates in the Sobolev spaces $H_{\varphi, x}^{s}$ of Proposition 2.37 in [2]. Moreover, since $b(\varphi, x)$ is odd $(\varphi, x)$, then $b(\varphi, x)|D|^{\frac{1}{2}}$ is reversibility preserving as well as $\boldsymbol{\Phi}(\varphi)$. Finally, note that $\Phi \pi_{0}=\pi_{0}=\Phi^{-1} \pi_{0}$, which implies that

$$
\boldsymbol{\Phi}^{-1} \Pi_{0} \boldsymbol{\Phi}=\Pi_{0} \boldsymbol{\Phi} .
$$

By the Lie expansion (3.16), we have

$$
\begin{aligned}
\Phi^{-1} P_{6} \Phi & =P_{6}-\mathrm{i}\left[A, P_{6}\right]-\frac{1}{2}\left[A,\left[A, P_{6}\right]\right]+\sum_{n=3}^{2 M+2} \frac{(-\mathrm{i})^{n}}{n !} \operatorname{ad}_{A(\varphi)}^{n}\left(P_{6}\right)+T_{M}, \\
T_{M} & :=\frac{(-\mathrm{i})^{2 M+3}}{(2 M+2) !} \int_{0}^{1}(1-\tau)^{2 M+2} \Phi^{-\tau}(\varphi) \operatorname{ad}_{A(\varphi)}^{2 M+3}\left(P_{6}\right) \Phi^{\tau}(\varphi) \mathrm{d} \tau,
\end{aligned}
$$

and, by (3.17),

$$
\begin{aligned}
& \Phi^{-1} \circ \omega \cdot \partial_{\varphi} \circ \Phi=\omega \cdot \partial_{\varphi}+\mathrm{i}\left(\omega \cdot \partial_{\varphi} A\right)(\varphi)-\sum_{n=2}^{2 M+1} \frac{(-\mathrm{i})^{n}}{n !} \operatorname{ad}_{A(\varphi)}^{n-1}\left(\omega \cdot \partial_{\varphi} A(\varphi)\right)+T_{M}^{\prime}, \\
& T_{M}^{\prime}:=-\frac{(-\mathrm{i})^{2 M+2}}{(2 M+1) !} \int_{0}^{1}(1-\tau)^{2 M+1} \Phi^{-\tau}(\varphi) \operatorname{ad}_{A(\varphi)}^{2 M+1}\left(\omega \cdot \partial_{\varphi} A(\varphi)\right) \Phi^{\tau}(\varphi) \mathrm{d} \tau .
\end{aligned}
$$

Note that $\operatorname{ad}_{A(\varphi)}^{2 M+3}\left(P_{6}\right)$ and $\operatorname{ad}_{A(\varphi)}^{2 M+1}\left(\omega \cdot \partial_{\varphi} A(\varphi)\right)$ are in OP $S^{-M}$. The number $M$ will be fixed in (8.5). Note also that in the expansions (7.129), (7.130) the operators have decreasing order and size. The terms of order 1 come from (7.129), in particular from $P_{6}-\mathrm{i}\left[A, P_{6}\right]$. Recalling (7.125), that $A(\varphi):=b(\varphi, x)|D|^{\frac{1}{2}},(3.26)$ and that (cfr. (4.1), (4.5))

$$
\Omega(\kappa, \xi)=\sqrt{\kappa}|\xi|^{\frac{3}{2}} \chi(\xi)+r_{0}(\kappa, \xi), \quad r_{0}(\kappa, \xi) \in S^{0},
$$

(the cut-off function $\chi$ is defined in (3.10)) we deduce that

$$
\left[A, P_{6}\right]=\mathrm{i} \frac{3}{2} \sqrt{\kappa} \mathrm{m}_{\frac{3}{2}} b_{x} \partial_{x}+\left(\frac{1}{2}\left(a_{1}^{(d)}\right)_{x} b-a_{1}^{(d)} b_{x}\right)|D|^{\frac{1}{2}}+\mathrm{Op}\left(r_{b, 0}\right),
$$

where $r_{b, 0} \in S^{0}$ is small with $b$. As a consequence, the first order term of $P_{6}-$ $\mathrm{i}\left[A, P_{6}\right]$ is $\left(a_{1}^{(d)}+\frac{3}{2} \sqrt{\kappa} \mathrm{m}_{\frac{3}{2}} b_{x}\right) \partial_{x}$ and we choose $b(\varphi, x)$ so that it is independent of $x$ : we look for a solution

$$
b(\varphi, x)=b_{1}(\varphi, x)+b_{2}(\varphi)
$$

of the equation

$$
a_{1}^{(d)}(\varphi, x)+\frac{3}{2} \mathrm{~m}_{\frac{3}{2}} \sqrt{\kappa} b_{x}(\varphi, x)=\left\langle a_{1}^{(d)}\right\rangle_{x}(\varphi):=\frac{1}{2 \pi} \int_{\mathbb{T}} a_{1}^{(d)}(\varphi, x) \mathrm{d} x .
$$


Therefore

$$
b_{1}(\varphi, x):=-\frac{2}{3 \mathrm{~m}_{\frac{3}{2}} \sqrt{\kappa}} \partial_{x}^{-1}\left(a_{1}^{(d)}(\varphi, x)-\left\langle a_{1}^{(d)}\right\rangle_{x}(\varphi)\right) .
$$

We now determine $b_{2}(\varphi)$ by imposing a condition at the order $1 / 2$. We deduce by (7.129), (7.130), (7.125), (7.132)-(7.134), that

$$
\begin{aligned}
L_{7}:=\Phi^{-1}(\varphi)\left(\omega \cdot \partial_{\varphi}+P_{6}\right) \Phi(\varphi)=\omega \cdot \partial_{\varphi}+\mathrm{im} \frac{3}{2} \Omega(\kappa, D) \\
\quad+\left\langle a_{1}^{(d)}\right\rangle_{x}(\varphi) \partial_{x}+\mathrm{i} a_{2}^{(d)}|D|^{\frac{1}{2}}+\mathrm{Op}\left(r_{7}^{(d)}\right)+T_{M}+T_{M}^{\prime},
\end{aligned}
$$

where $a_{2}^{(d)}(\varphi, x)$ is the real function

$$
\begin{aligned}
a_{2}^{(d)}:= & -\frac{1}{2}\left(a_{1}^{(d)}\right)_{x} b_{1}+a_{1}^{(d)}\left(b_{1}\right)_{x}+\frac{3}{4} \sqrt{\kappa} \mathrm{m}_{\frac{3}{2}}\left(\left(b_{1}\right)_{x}^{2}-\frac{1}{2}\left(b_{1}\right)_{x x} b_{1}\right) \\
& +\left(\omega \cdot \partial_{\varphi} b_{1}\right)-\left(\frac{1}{2}\left(a_{1}^{(d)}\right)_{x}+\frac{3}{8} \sqrt{\kappa} \mathrm{m}_{\frac{3}{2}}\left(b_{1}\right)_{x x}\right) b_{2}+\left(\omega \cdot \partial_{\varphi} b_{2}\right)
\end{aligned}
$$

and

$$
\begin{aligned}
& \mathrm{Op}\left(r_{7}^{(d)}\right):=\mathrm{Op}\left(-\mathrm{i} r_{b, 0}+r_{b,-\frac{1}{2}}+r_{6}^{(d)}\right) \\
& -\frac{1}{2}\left[b|D|^{\frac{1}{2}},\left(\frac{1}{2}\left(a_{1}^{(d)}\right)_{x} b-a_{1}^{(d)} b_{x}\right)|D|^{\frac{1}{2}}+\mathrm{Op}\left(r_{b, 0}\right)\right] \\
& \quad+\sum_{n=3}^{M-1} \frac{(-\mathrm{i})^{n}}{n !} \operatorname{ad}_{A(\varphi)}^{n}\left(P_{6}\right)-\sum_{n=2}^{M} \frac{(-\mathrm{i})^{n}}{n !} \operatorname{ad}_{A(\varphi)}^{n-1}\left(\omega \cdot \partial_{\varphi} A(\varphi)\right) \in \mathrm{OP} S^{0},
\end{aligned}
$$

where $r_{b,-\frac{1}{2}} \in S^{-\frac{1}{2}}$ is small in $b$. In view of Section 7.6 we now determine the function $b_{2}(\varphi)$ so that the space average of the function $a_{2}^{(d)}$ in (7.137) is independent of $\varphi$, that is

$$
\left\langle a_{2}^{(d)}\right\rangle_{x}(\varphi)=\mathrm{m}_{\frac{1}{2}} \in \mathbb{R}, \quad \forall \varphi \in \mathbb{T}^{\nu}
$$

Noting that the space average $\left\langle\left(\frac{1}{2}\left(a_{1}^{(d)}\right)_{x}+\frac{3}{8} \mathrm{~m}_{\frac{3}{2}} \sqrt{\kappa}\left(b_{1}\right)_{x x}\right) b_{2}(\varphi)\right\rangle_{x}=0$ and that $\left\langle\omega \cdot \partial_{\varphi} b_{1}\right\rangle_{\varphi, x}=0$, we get

$$
\begin{aligned}
& \mathrm{m}_{\frac{1}{2}}:=\langle-\left.\frac{1}{2}\left(a_{1}^{(d)}\right)_{x} b_{1}+a_{1}^{(d)}\left(b_{1}\right)_{x}+\frac{3}{4} \sqrt{\kappa} \mathrm{m}_{\frac{3}{2}}\left(\left(b_{1}\right)_{x}^{2}-\frac{1}{2}\left(b_{1}\right)_{x x} b_{1}\right)\right\rangle_{\varphi, x}, \\
& b_{2}(\varphi):=-\left(\omega \cdot \partial_{\varphi}\right)_{\operatorname{ext}}^{-1}\left(\left\langle-\frac{1}{2}\left(a_{1}^{(d)}\right)_{x} b_{1}+a_{1}^{(d)}\left(b_{1}\right)_{x}+\right.\right. \\
&\left.\left.+\frac{3}{4} \mathrm{~m}_{\frac{3}{2}} \sqrt{\kappa}\left(\left(b_{1}\right)_{x}^{2}-\frac{1}{2}\left(b_{1}\right)_{x x} b_{1}\right)+\left(\omega \cdot \partial_{\varphi} b_{1}\right)\right\rangle_{x}-\mathrm{m}_{\frac{1}{2}}\right) .
\end{aligned}
$$

Note that (7.139) holds for any $\omega \in \operatorname{DC}(v, \tau)$. 
Time reduction. In order to remove the $\varphi$-dependence of the coefficient $\left\langle a_{1}^{(d)}\right\rangle_{x}(\varphi)$ of the first order term of the operator $L_{7}$ in (7.136), we conjugate $L_{7}$ with the map

$$
(\mathcal{V} u)(\varphi, x):=u(\varphi, x+\varrho(\varphi)),
$$

where $\varrho(\varphi)$ is a real periodic function to be chosen, see (7.145). Note that $\mathcal{V}$ is a particular case of the transformation $\mathcal{E}$ in (7.34) for a function $\beta(\varphi, x)=\varrho(\varphi)$, independent of $x$. We have that

$$
\mathcal{V}^{-1}\left(\omega \cdot \partial_{\varphi}\right) \mathcal{V}=\omega \cdot \partial_{\varphi}+\left(\omega \cdot \partial_{\varphi} \varrho\right) \partial_{x},
$$

whereas the Fourier multipliers are left unchanged and a pseudodifferential operator of symbol $a(\varphi, x, \xi)$ transforms as

$$
\mathcal{V}^{-1} \mathrm{Op}(a(\varphi, x, \xi)) \mathcal{V}=\operatorname{Op}(a(\varphi, x-\varrho(\varphi), \xi))
$$

We choose $\varrho(\varphi)$ such that

$$
\omega \cdot \partial_{\varphi} \varrho(\varphi)+\left\langle a_{1}^{(d)}\right\rangle_{x}(\varphi)=\mathrm{m}_{1}, \quad \mathrm{~m}_{1}:=\left\langle a_{1}^{(d)}\right\rangle_{\varphi, x} \in \mathbb{R},
$$

(where $a_{1}^{(d)}$ is fixed in Lemma 7.9), namely we define

$$
\varrho(\varphi):=-\left(\omega \cdot \partial_{\varphi}\right)_{\mathrm{ext}}^{-1}\left(\left\langle a_{1}^{(d)}\right\rangle_{x}-\mathrm{m}_{1}\right) .
$$

Note that (7.144) holds for any $\omega \in \operatorname{DC}(v, \tau)$.

We sum up these two transformations into the following lemma.

Lemma 7.12. Let $M \in \mathbb{N}$, $q_{0} \in \mathbb{N}_{0}$. Let $b(\varphi, x)=b_{1}(\varphi, x)+b_{2}(\varphi)$ and $\varrho(\varphi)$ be the functions defined respectively in (7.135), (7.141), (7.145). Then, conjugating $\mathcal{L}_{6}$ in (7.108) via the invertible, real, reversibility preserving and momentum preserving maps $\boldsymbol{\Phi}, \mathcal{V}$ defined in (7.126)-(7.127) and (7.142), we obtain, for any $\omega \in \mathrm{DC}(v, \tau)$, the real, reversible and momentum preserving operator

$$
\begin{aligned}
\mathcal{L}_{8} & :=\mathcal{V}^{-1} \boldsymbol{\Phi}^{-1} \mathcal{L}_{6} \boldsymbol{\Phi} \mathcal{V} \\
& =\omega \cdot \partial_{\varphi}+\mathrm{im}_{\frac{3}{2}} \boldsymbol{\Omega}(\kappa, D)+\mathrm{m}_{1} \partial_{x}+\mathrm{iA}_{3}^{(d)}|D|^{\frac{1}{2}}+\mathrm{i} \Pi_{0}+\mathbf{R}_{8}^{(0, d)}+\mathbf{T}_{8, M},
\end{aligned}
$$

where:

1. the real constant $\mathrm{m}_{1}$ defined in (7.144) satisfies $\left|\mathrm{m}_{1}\right|^{k_{0}, v} \lesssim \varepsilon$;

2. $\mathbf{A}_{3}^{(d)}$ is a diagonal matrix of multiplication

$$
\mathbf{A}_{3}^{(d)}:=\left(\begin{array}{cc}
a_{3}^{(d)} & 0 \\
0 & a_{3}^{(d)}
\end{array}\right),
$$

for a real function $a_{3}^{(d)}$ which is a quasi-periodic traveling wave, even $(\varphi, x)$, satisfying

$$
\left\langle a_{3}^{(d)}\right\rangle_{x}(\varphi)=\mathrm{m}_{\frac{1}{2}} \in \mathbb{R}, \quad \forall \varphi \in \mathbb{T}^{v},
$$

where $\mathrm{m}_{\frac{1}{2}} \in \mathbb{R}$ is the constant in (7.140), and for some $\sigma=\sigma\left(\tau, v, k_{0}\right)>0$, for all $s \geqq s_{0}$,

$$
\left\|a_{3}^{(d)}\right\|_{s}^{k_{0}, v} \lesssim_{s} \varepsilon v^{-1}\left(1+\left\|\Im_{0}\right\|_{s+\sigma}^{k_{0}, v}\right)
$$


3. $\mathbf{R}_{8}^{(0, d)}$ is a block-diagonal operator

$$
\mathbf{R}_{8}^{(0, d)}=\left(\begin{array}{cc}
r_{8}^{(d)}(\varphi, x, D) & 0 \\
0 & r_{8}^{(d)}(\varphi, x, D)
\end{array}\right) \in \operatorname{OPS}^{0},
$$

that satisfies for all $\alpha \in \mathbb{N}_{0}$, for some $\sigma_{M}(\alpha):=\sigma_{M}\left(k_{0}, \tau, \nu, \alpha\right)>0$ and for all $s \geqq s_{0}$,

$$
\left\|\mathbf{R}_{8}^{(0, d)}\right\|_{0, s, \alpha}^{k_{0}, v} \lesssim_{s, M, \alpha} \varepsilon v^{-1}\left(1+\left\|\Im_{0}\right\|_{s+\sigma_{M}(\alpha)}^{k_{0}, v}\right) ;
$$

4. For any $\mathrm{q} \in \mathbb{N}_{0}^{v}$ with $|\mathrm{q}| \leqq \mathrm{q}_{0}, n_{1}, n_{2} \in \mathbb{N}_{0}$ with $n_{1}+n_{2} \leqq M-2\left(k_{0}+\right.$ $\left.\mathrm{q}_{0}\right)+\frac{5}{2}$, the operator $\langle D\rangle^{n_{1}} \partial_{\varphi}^{\mathrm{q}} \mathbf{T}_{8, M}(\varphi)\langle D\rangle^{n_{2}}$ is $\mathcal{D}^{k_{0}}$-tame with a tame constant satisfying, for some $\sigma_{M}\left(\mathrm{q}_{0}\right):=\sigma_{M}\left(k_{0}, \tau, v, \mathrm{q}_{0}\right)$, for any $s_{0} \leqq s \leqq S$,

$$
\mathfrak{M}_{\langle D\rangle^{n_{1}} \partial_{\varphi}^{\mathrm{q}} \mathbf{T}_{8, M}(\varphi)\langle D\rangle^{n_{2}}}(s) \lesssim S, M, \mathrm{q}_{0} \varepsilon v^{-1}\left(1+\left\|\mathfrak{I}_{0}\right\|_{S+\sigma_{M}\left(\mathrm{q}_{0}\right)}^{k_{0}, v}\right)
$$

5. The operators $\boldsymbol{\Phi}^{ \pm 1}-\mathrm{Id},\left(\boldsymbol{\Phi}^{ \pm 1}-\mathrm{Id}\right)^{*}$ are $\mathcal{D}^{k_{0}}-\frac{1}{2}\left(k_{0}+1\right)$-tame and the operators $\mathcal{V}^{ \pm 1}-\mathrm{Id},\left(\mathcal{V}^{ \pm 1}-\mathrm{Id}\right)^{*}$ are $\mathcal{D}^{k_{0}}-\left(k_{0}+2\right)$-tame, with tame constants satisfying, for some $\sigma>0$ and for all $s_{0} \leqq s \leqq S$,

$$
\begin{aligned}
& \mathfrak{M}_{\Phi^{ \pm 1}-\mathrm{Id}}(s)+\mathfrak{M}_{\left(\Phi^{ \pm 1}-\mathrm{Id}\right)^{*}}(s) \lesssim S \varepsilon v^{-1}\left(1+\left\|\Im_{0}\right\|_{S+\sigma}^{k_{0}, v}\right), \\
& \mathfrak{M}_{\mathcal{V}^{ \pm 1}-\mathrm{Id}}(s)+\mathfrak{M}_{\left(\mathcal{V}^{ \pm 1}-\mathrm{Id}\right)^{*}}(s) \lesssim s \varepsilon v^{-1}\left(1+\left\|\Im_{0}\right\|_{s+\sigma}^{k_{0}, v}\right) .
\end{aligned}
$$

Furthermore, for any $s_{1}$ as in (7.15), $\alpha \in \mathbb{N}_{0}$, $q \in \mathbb{N}_{0}^{v}$, with $|\mathrm{q}| \leqq \mathrm{q}_{0}$, and $n_{1}, n_{2} \in \mathbb{N}_{0}$, with $n_{1}+n_{2} \leqq M-2 q_{0}+\frac{1}{2}$, we have

$$
\begin{aligned}
& \left\|\Delta_{12} a_{3}^{(d)}\right\|_{s_{1}} \lesssim_{s_{1}} \varepsilon v^{-1}\left\|i_{1}-i_{2}\right\|_{s_{1}+\sigma},\left|\Delta_{12} \mathrm{~m}_{1}\right| \lesssim \varepsilon\left\|i_{1}-i_{2}\right\|_{s_{0}+\sigma} \\
& \left\|\Delta_{12} \mathbf{R}_{8}^{(0, d)}\right\|_{0, s_{1}, \alpha} \lesssim_{s_{1}, M, \alpha} \varepsilon v^{-1}\left\|i_{1}-i_{2}\right\|_{s_{1}+\sigma_{M}(\alpha)} \\
& \left\|\langle D\rangle^{n_{1}} \partial_{\varphi}^{\mathrm{q}} \Delta_{12} \mathbf{T}_{8, M}\langle D\rangle^{n_{2}}\right\|_{\mathcal{L}\left(H^{s_{1}}\right)} \lesssim_{s_{1}, M, \mathrm{q}_{0}} \varepsilon v^{-1}\left\|i_{1}-i_{2}\right\|_{s_{1}+\sigma_{M}\left(\mathrm{q}_{0}\right)} \\
& \left\|\Delta_{12}(\mathcal{A}) h\right\|_{s_{1}} \lesssim_{s_{1}} \varepsilon v^{-1}\left\|i_{1}-i_{2}\right\|_{s_{1}+\sigma}\|h\|_{s_{1}+\sigma}, \quad \mathcal{A} \in\left\{\boldsymbol{\Phi}^{ \pm 1},\left(\boldsymbol{\Phi}^{ \pm 1}\right)^{*}, \mathcal{V}^{ \pm 1},\left(\mathcal{V}^{ \pm 1}\right)^{*}\right\}
\end{aligned}
$$

Proof. The function $b(\varphi, x)=b_{1}(\varphi, x)+b_{2}(\varphi)$, with $b_{1}$ and $b_{2}$, defined in (7.135) and (7.141) and the function $\varrho(\varphi)$ in (7.145), satisfy, by Lemma 3.8 and (7.95), $\left\|b_{1}\right\|_{s}^{k_{0}, v} \lesssim_{s} \varepsilon\left(1+\left\|\Im_{0}\right\|_{s+\sigma}^{k_{0}, v}\right), \quad\|b\|_{s}^{k_{0}, v},\left\|b_{2}\right\|_{s}^{k_{0}, v},\|\varrho\|_{s}^{k_{0}, v} \lesssim_{s} \varepsilon v^{-1}\left(1+\left\|\Im_{0}\right\|_{s+\sigma}^{k_{0}, v}\right)$

for some $\sigma>0$ and for all $s \geqq s_{0}$. The estimate $\left|\mathrm{m}_{1}\right|^{k_{0}, v} \lesssim \varepsilon$ follows by (7.144) and (7.95). The function

$$
a_{3}^{(d)}(\varphi, x):=\mathcal{V}^{-1}\left(a_{2}^{(d)}\right)=a_{2}^{(d)}(\varphi, x-\varrho(\varphi)),
$$

where $a_{2}^{(d)}$ is defined in (7.137), satisfies (7.147) by (7.139). Moreover, the estimate (7.148) follows by Lemma 3.8 and (7.95), (7.157). The estimate (7.149) for (cfr. (7.143))

$$
r_{8}^{(d)}(\varphi, x, D):=\mathcal{V}^{-1} r_{7}^{(d)}(\varphi, x, D) \mathcal{V}=r_{7}^{(d)}(\varphi, x-\varrho(\varphi), D)
$$


with $r_{7}^{(d)}$ defined in (7.138), follows by Lemmata 3.5, 3.6, 3.8 and (7.157), (7.110). The smoothing term $\mathbf{T}_{8, M}$ in (7.146) is, using also (7.128),

$$
\begin{aligned}
\mathbf{T}_{8, M}:= & \mathcal{V}^{-1}\left(\boldsymbol{\Phi}^{-1} \mathbf{T}_{6, M} \boldsymbol{\Phi}+\mathrm{i} \Pi_{0}(\boldsymbol{\Phi}-\mathrm{Id})+\boldsymbol{\Phi}^{-1} \mathbf{R}_{6}^{(-M, o)} \boldsymbol{\Phi}\right) \mathcal{V} \\
& +\mathcal{V}^{-1}\left(\begin{array}{cc}
T_{M}+T_{M}^{\prime} & 0 \\
0 & \overline{T_{M}}+\overline{T_{M}^{\prime}}
\end{array}\right) \mathcal{V}
\end{aligned}
$$

with $T_{M}$ and $T_{M}^{\prime}$ defined in (7.129), (7.130). The estimate (7.150) follows by (7.125), Lemmata 3.12, 3.13, the tame estimates of $\boldsymbol{\Phi}$ in Proposition 2.37 in [2], and estimates (7.95), (7.157), (7.151), (7.111), noting that operators of the form $\partial_{\lambda}^{k} \partial_{\varphi}^{q} \mathcal{V}^{ \pm 1}$ lose $|k|+|q|$ derivatives. The estimate (7.151) follows by Lemma 2.38 in [2] and (7.157), whereas (7.152) follows by the equivalent representation for $\mathcal{V}$ as in (7.104), Lemmata 3.12, 3.8 and (7.157). The estimates (7.153), (7.154), (7.155), (7.156) are proved in the same fashion. By Lemma 7.9-3, the function $a_{1}^{(d)}$ is an even $(\varphi, x)$ quasi-periodic traveling wave, hence the function $b_{1}$ in (7.135) is an $\operatorname{odd}(\varphi, x)$ quasi-periodic traveling wave, the function $b_{2}$ in (7.141) is odd in $\varphi$ and satisfies $b_{2}(\varphi-\vec{J} \varsigma)=b_{2}(\varphi)$ for all $\varsigma \in \mathbb{R}$, whereas the function $\varrho$ in (7.145) is odd in $\varphi$ and satisfies $\varrho(\varphi-\vec{\jmath} \varsigma)=\varrho(\varphi)$ for all $\varsigma \in \mathbb{R}$. By Lemmata 3.19, 3.29, and 3.25, the transformations $\boldsymbol{\Phi}$ and $\mathcal{V}$ are reversibility and momentum preserving. Then the operator $\mathcal{L}_{8}$ is reversible and momentum preserving. The function $a_{3}^{(d)}$ is an even $(\varphi, x)$ quasi-periodic traveling wave.

\subsection{Reduction of the Order $1 / 2$}

The goal of this section is to transform the operator $\mathcal{L}_{8}$ in (7.146) into the operator $\mathcal{L}_{9}$ in (7.168) whose coefficient in front of $|D|^{1 / 2}$ is a constant. We eliminate the $x$-dependence and, in view of the property (7.147), we obtain that this transformation removes also the $\varphi$-dependence.

We first write the operator $\mathcal{L}_{8}$ in (7.146) as

$$
\mathcal{L}_{8}=\omega \cdot \partial_{\varphi}+\left(\begin{array}{cc}
P_{8} & 0 \\
0 & \frac{P_{8}}{P}
\end{array}\right)+\mathrm{i} \boldsymbol{\Pi}_{0}+\mathbf{T}_{8, M},
$$

where

$$
P_{8}:=\operatorname{im}_{\frac{3}{2}} \Omega(\kappa, D)+\mathrm{m}_{1} \partial_{x}+\mathrm{i} a_{3}^{(d)}|D|^{\frac{1}{2}}+\mathrm{Op}\left(r_{8}^{(d)}\right) .
$$

We conjugate $\mathcal{L}_{8}$ through the real operator

$$
\boldsymbol{\Psi}(\varphi):=\left(\begin{array}{cc}
\Psi(\varphi) & 0 \\
0 & \Psi(\varphi)
\end{array}\right),
$$

where $\Psi(\varphi):=\left.\Psi^{\tau}(\varphi)\right|_{\tau=1}$ is the time-1 flow of

$$
\left\{\begin{array}{l}
\partial_{\tau} \Psi^{\tau}(\varphi)=B(\varphi) \Psi^{\tau}(\varphi), \quad B(\varphi):=b_{3}(\varphi, x) \mathcal{H}, \\
\Psi^{0}(\varphi)=\mathrm{Id}
\end{array}\right.
$$


the function $b_{3}(\varphi, x)$ is a smooth, real, periodic function to be chosen later (see (7.165)) and $\mathcal{H}$ is the Hilbert transform defined in (3.19). Note that $\Psi \pi_{0}=\pi_{0}=$ $\Psi^{-1} \pi_{0}$, so that

$$
\boldsymbol{\Psi}^{-1} \Pi_{0} \Psi=\Pi_{0} \Psi
$$

By the Lie expansion in (3.16) we have

$$
\begin{aligned}
\Psi^{-1} P_{8} \Psi & =P_{8}-\left[B, P_{8}\right]+\sum_{n=2}^{M+1} \frac{(-1)^{n}}{n !} \operatorname{ad}_{B(\varphi)}^{n}\left(P_{8}\right)+L_{M}, \\
L_{M} & :=\frac{(-1)^{M+2}}{(M+1) !} \int_{0}^{1}(1-\tau)^{M+1} \Psi^{-\tau}(\varphi) \operatorname{ad}_{B(\varphi)}^{M+2}\left(P_{8}\right) \Psi^{\tau}(\varphi) \mathrm{d} \tau,
\end{aligned}
$$

and, by (3.17),

$$
\begin{aligned}
& \Psi^{-1} \circ \omega \cdot \partial_{\varphi} \circ \Psi= \\
& \omega \cdot \partial_{\varphi}+\left(\omega \cdot \partial_{\varphi} B(\varphi)\right)-\sum_{n=2}^{M} \frac{(-1)^{n}}{n !} \operatorname{ad}_{B(\varphi)}^{n-1}\left(\omega \cdot \partial_{\varphi} B(\varphi)\right)+L_{M}^{\prime}, \\
& L_{M}^{\prime}:=\frac{(-1)^{M}}{M !} \int_{0}^{1}(1-\tau)^{M} \Psi^{-\tau}(\varphi) \operatorname{ad}_{B(\varphi)}^{M}\left(\omega \cdot \partial_{\varphi} B(\varphi)\right) \Psi^{\tau}(\varphi) \mathrm{d} \tau .
\end{aligned}
$$

The number $M$ will be fixed in (8.5). The contributions at order $1 / 2$ come from (7.162), in particular from $P_{8}-\left[B, P_{8}\right]$ (recall (7.158)). Since $B=b_{3} \mathcal{H}$ (see (7.160)), by (3.26) and (7.131) we have

$$
\begin{aligned}
P_{8}-\left[B, P_{8}\right]= & \mathrm{im}_{\frac{3}{2}} \Omega(\kappa, D)+\mathrm{m}_{1} \partial_{x}+\mathrm{i}\left(a_{3}^{(d)}-\frac{3}{2} \mathrm{~m}_{\frac{3}{2}} \sqrt{\kappa}\left(b_{3}\right)_{x}\right)|D|^{\frac{1}{2}} \\
& +\mathrm{Op}\left(r_{8}^{(d)}+r_{b_{3},-\frac{1}{2}}\right)-\left[B, \mathrm{~m}_{1} \partial_{x}+\mathrm{i} a_{3}^{(d)}|D|^{\frac{1}{2}}+\mathrm{Op}\left(r_{8}^{(d)}\right)\right],
\end{aligned}
$$

where $\mathrm{Op}\left(r_{b_{3},-\frac{1}{2}}\right) \in \mathrm{OP} S^{-\frac{1}{2}}$ is small with $b_{3}$. Recalling that, by (7.147), the space average $\left\langle a_{3}^{(d)}\right\rangle_{x}(\varphi)=\mathrm{m}_{\frac{1}{2}}$ for all $\varphi \in \mathbb{T}^{\nu}$, we choose the function $b_{3}(\varphi, x)$ such that $a_{3}^{(d)}-\frac{3}{2} \mathrm{~m}_{\frac{3}{2}} \sqrt{\kappa}\left(b_{3}\right)_{x}=\mathrm{m}_{\frac{1}{2}}$, namely

$$
b_{3}(\varphi, x):=\frac{2}{3 \mathrm{~m}_{\frac{3}{2}} \sqrt{\kappa}} \partial_{x}^{-1}\left(a_{3}^{(d)}(\varphi, x)-\left\langle a_{3}^{(d)}\right\rangle_{x}(\varphi)\right), \quad\left\langle a_{3}^{(d)}\right\rangle_{x}(\varphi)=\mathrm{m}_{\frac{1}{2}} .
$$

We deduce by (7.162)-(7.163) and (7.164), (7.165) that

$$
\begin{aligned}
L_{9} & :=\Psi^{-1}(\varphi)\left(\omega \cdot \partial_{\varphi}+P_{8}\right) \Psi(\varphi) \\
& =\omega \cdot \partial_{\varphi}+\mathrm{im}_{\frac{3}{2}} \Omega(\kappa, D)+\mathrm{m}_{1} \partial_{x}+\mathrm{im}_{\frac{1}{2}}|D|^{\frac{1}{2}}+\mathrm{Op}\left(r_{9}^{(d)}\right)+L_{M}+L_{M}^{\prime},
\end{aligned}
$$


where

$$
\begin{aligned}
\mathrm{Op}\left(r_{9}^{(d)}\right):= & \operatorname{Op}\left(r_{8}^{(d)}+r_{b_{3},-\frac{1}{2}}\right)-\left[B(\varphi), \mathrm{m}_{1} \partial_{x}+\mathrm{i} a_{3}^{(d)}|D|^{\frac{1}{2}}+\mathrm{Op}\left(r_{8}^{(d)}\right)\right]+\left(\omega \cdot \partial_{\varphi} B(\varphi)\right) \\
& +\sum_{n=2}^{M-1} \frac{(-1)^{n}}{n !} \operatorname{ad}_{B(\varphi)}^{n}\left(P_{8}\right)-\sum_{n=2}^{M} \frac{(-1)^{n}}{n !} \operatorname{ad}_{B(\varphi)}^{n-1}\left(\omega \cdot \partial_{\varphi} B(\varphi)\right) \in \operatorname{OPS}^{0}
\end{aligned}
$$
lemma.

Define the matrix $\Sigma:=\left(\begin{array}{cc}1 & 0 \\ 0 & -1\end{array}\right)$. Summing up, we have obtained the following

Lemma 7.13. Let $M \in \mathbb{N}$, $q_{0} \in \mathbb{N}_{0}$. Let $b_{3}$ be the function defined in (7.165). Then, conjugating the operator $\mathcal{L}_{8}$ in (7.146) via the invertible, real, reversibility and momentum preserving map $\boldsymbol{\Psi}$ defined in (7.159), (7.160), we obtain, for any $\omega \in \mathrm{DC}(v, \tau)$, the real, reversible and momentum preserving operator

$$
\mathcal{L}_{9}:=\Psi^{-1} \mathcal{L}_{8} \boldsymbol{\Psi}=\omega \cdot \partial_{\varphi}+\mathrm{im}_{\frac{3}{2}} \boldsymbol{\Omega}(\kappa, D)+\mathrm{m}_{1} \partial_{x}+\mathrm{im}_{\frac{1}{2}} \boldsymbol{\Sigma}|D|^{\frac{1}{2}}+\mathrm{i} \Pi_{0}+\mathbf{R}_{9}^{(0, d)}+\mathbf{T}_{9, M},
$$

where

1. the constant $\mathrm{m}_{\frac{1}{2}}$ defined in (7.140) satisfies $\left|\mathrm{m}_{\frac{1}{2}}\right|^{k_{0}, v} \lesssim \varepsilon^{2}$;

2. $\mathbf{R}_{9}^{(0, d)}$ is a block-diagonal operator

$$
\mathbf{R}_{9}^{(0, d)}=\left(\begin{array}{cc}
r_{9}^{(d)}(\varphi, x, D) & 0 \\
0 & r_{9}^{(d)}(\varphi, x, D)
\end{array}\right) \in \mathrm{OPS}^{0}
$$

that satisfies, for some $\sigma_{M}:=\sigma_{M}\left(k_{0}, \tau, v\right)>0$, and for all $s \geqq s_{0}$,

$$
\left\|\mathbf{R}_{9}^{(0, d)}\right\|_{0, s, 1}^{k_{0}, v} \lesssim_{s, M} \varepsilon v^{-1}\left(1+\left\|\Im_{0}\right\|_{s+\sigma_{M}}^{k_{0}, v}\right) ;
$$

3. For any $\mathrm{q} \in \mathbb{N}_{0}^{v}$ with $|\mathrm{q}| \leqq \mathrm{q}_{0}, n_{1}, n_{2} \in \mathbb{N}_{0}$ with $n_{1}+n_{2} \leqq M-2\left(k_{0}+\right.$ $\left.\mathrm{q}_{0}\right)+\frac{5}{2}$, the operator $\langle D\rangle^{n_{1}} \partial_{\varphi}^{q_{\varphi}} \mathbf{T}_{9, M}(\varphi)\langle D\rangle^{n_{2}}$ is $\mathcal{D}^{k_{0}}$-tame with a tame constant satisfying, for some $\sigma_{M}\left(\mathrm{q}_{0}\right):=\sigma_{M}\left(k_{0}, \tau, \nu, \mathrm{q}_{0}\right)$, for any $s_{0} \leqq s \leqq S$,

$$
\mathfrak{M}_{\langle D\rangle^{n_{1}} \partial_{\varphi}^{\mathrm{q}} \mathbf{T}_{9, M}(\varphi)\langle D\rangle^{n_{2}}}(s) \lesssim S, M, \mathrm{q}_{0} \varepsilon v^{-1}\left(1+\left\|\mathfrak{I}_{0}\right\|_{s+\sigma_{M}\left(\mathrm{q}_{0}\right)}^{\left.k_{0}\right)}\right)
$$

4. The operators $\Psi^{ \pm 1}-\mathrm{Id},\left(\Psi^{ \pm 1}-\mathrm{Id}\right)^{*}$ are $\mathcal{D}^{k_{0}}$-tame, with tame constants satisfying, for some $\sigma:=\sigma\left(k_{0}, \tau, \nu\right)>0$ and for all $s \geqq s_{0}$,

$$
\mathfrak{M}_{\Psi^{ \pm 1}-\mathrm{Id}}(s)+\mathfrak{M}_{\left(\Psi^{ \pm 1}-\mathrm{Id}\right)^{*}}(s) \lesssim s \varepsilon v^{-1}\left(1+\left\|\mathfrak{I}_{0}\right\|_{s+\sigma}^{k_{0}, v}\right) .
$$

Furthermore, for any $s_{1}$ as in (7.15), $\alpha \in \mathbb{N}_{0}$, $q \in \mathbb{N}_{0}^{v}$, with $|\mathrm{q}| \leqq q_{0}$, and $n_{1}, n_{2} \in \mathbb{N}_{0}$, with $n_{1}+n_{2} \leqq M-2 q_{0}+\frac{1}{2}$, we have

$$
\begin{aligned}
& \left\|\Delta_{12} \mathbf{R}_{9}^{(0, d)}\right\|_{0, s_{1}, 1} \lesssim s_{1}, M \varepsilon v^{-1}\left\|i_{1}-i_{2}\right\|_{s_{1}+\sigma_{M}},\left|\Delta_{12} \mathrm{~m}_{\frac{1}{2}}\right| \lesssim \varepsilon^{2}\left\|i_{1}-i_{2}\right\|_{s_{0}+\sigma}, \\
& \left\|\langle D\rangle^{n_{1}} \partial_{\varphi}^{\mathrm{q}} \Delta_{12} \mathbf{T}_{9, M}\langle D\rangle^{n_{2}}\right\|_{\mathcal{L}_{\left(H^{s_{1}}\right)} \lesssim_{s_{1}, M, \mathrm{q}_{0}} \varepsilon v^{-1}\left\|i_{1}-i_{2}\right\|_{s_{1}+\sigma_{M}\left(\mathrm{q}_{0}\right)}}, \\
& \left\|\Delta_{12}\left(\boldsymbol{\Psi}^{ \pm 1}\right) h\right\|_{s_{1}}+\left\|\Delta_{12}\left(\boldsymbol{\Psi}^{ \pm 1}\right)^{*} h\right\|_{s_{1}} \lesssim_{s_{1}} \varepsilon v^{-1}\left\|i_{1}-i_{2}\right\|_{s_{1}+\sigma}\|h\|_{s_{1}+\sigma} \cdot
\end{aligned}
$$


Proof. The function $b_{3}(\varphi, x)$ defined in (7.165), satisfies, by (7.148) and the estimate of $m_{\frac{3}{2}}$ given in Lemma 7.9-2, for some $\sigma>0$ and for all $s \geqq s_{0}$,

$$
\left\|b_{3}\right\|_{s}^{k_{0}, v} \lesssim_{s} \varepsilon v^{-1}\left(1+\left\|\Im_{0}\right\|_{s+\sigma}^{k_{0}, v}\right) .
$$

The estimate for $m_{\frac{1}{2}}$ follows by (7.140), (3.7) and (7.95), (7.157). The estimate (7.169) follows by (7.167), (7.158), Lemmata 3.5, 3.6, and (7.148), (7.149), (7.175). By (7.146), (7.158), (7.166), and (7.161), the smoothing term $\mathbf{T}_{9, M}$ in (7.168) is

$$
\mathbf{T}_{9, M}:=\boldsymbol{\Psi}^{-1} \mathbf{T}_{8, M} \boldsymbol{\Psi}+\mathrm{i} \boldsymbol{\Pi}_{0}(\boldsymbol{\Psi}-\mathrm{Id})+\left(\begin{array}{cc}
L_{M}+L_{M}^{\prime} & 0 \\
0 & \overline{L_{M}}+\overline{L_{M}^{\prime}}
\end{array}\right)
$$

with $L_{M}$ and $L_{M}^{\prime}$ introduced in (7.162), (7.163). The estimate (7.170) follows by Lemmata 3.12, 3.13, 3.7, (7.158), (7.148), (7.150), (7.175), (7.171). The estimate (7.171) follows by Lemma 3.13 and (7.175). The estimates (7.172), (7.173), (7.174) are proved in the same fashion. By Lemma 7.12, the function $a_{3}^{(d)}$ is a even $(\varphi, x)$ quasi-periodic traveling wave. Hence the function $b_{3}$ in (7.165) is a odd $(\varphi, x)$ quasiperiodic traveling wave. By Lemmata 3.19, 3.29, and 3.25, the transformation $\Psi$ is reversibility and momentum preserving, therefore the operator $\mathcal{L}_{9}$ is reversible and momentum preserving.

Remark 7.14. In Proposition 7.18 we shall estimate $\left\|\left[\partial_{x}, \mathbf{R}_{9}^{(0, d)}\right]\right\|_{0, s, 0}^{k_{0}, v}$ using (7.169) and (3.27). In order to control $\left\|\mathbf{R}_{9}^{(0, d)}\right\|_{0, s, 1}^{k_{0}, v}$ we used the estimates (7.97) for finitely many $\alpha \in \mathbb{N}_{0}, \alpha \leqq \alpha(M)$, depending on $M$. Furthermore in Proposition 7.18 we shall use (7.172)-(7.173) only for $s_{1}=s_{0}$.

\subsection{Conclusion: Partial Reduction of $\mathcal{L}_{\omega}$}

By Sections 7.1-7.6, the linear operator $\mathcal{L}$ in (7.12) is semi-conjugated, for all $\omega \in \mathrm{DC}(v, \tau)$, to the real, reversible and momentum preserving operator $\mathcal{L}_{9}$ defined in (7.168), namely

$$
\mathcal{L}_{9}=\mathcal{W}_{2}^{-1} \mathcal{L} \mathcal{W}_{1}
$$

where

$$
\mathcal{W}_{1}:=\mathcal{P} \mathcal{Z} \mathcal{E} \mathcal{Q} \widetilde{\mathcal{M}} \mathcal{C} \boldsymbol{\Phi}_{M} \boldsymbol{\Phi} \mathcal{V} \boldsymbol{\Psi}, \quad \mathcal{W}_{2}:=\mathcal{P} \rho \mathcal{Z} \mathcal{E} \mathcal{Q} \widetilde{\mathcal{M}} \mathcal{C} \boldsymbol{\Phi}_{M} \boldsymbol{\Phi} \mathcal{V} \boldsymbol{\Psi}
$$

Moreover $\mathcal{L}_{9}$ is defined for all $\omega \in \mathbb{R}^{v}$.

Now we deduce a similar conjugation result for the projected operator $\mathcal{L}_{\omega}$ in (6.23), that is (7.1), which acts in the normal subspace $\mathfrak{H}_{\mathbb{S}^{+}, \Sigma}^{\angle}$. We first introduce some notation.

We denote by $\Pi_{\mathbb{S}^{+}, \Sigma}^{\top}$ and $\Pi_{\mathbb{S}^{+}, \Sigma}^{\angle}$ the projections on the subspaces $\mathfrak{H}_{\mathbb{S}^{+}, \Sigma}^{\top}$ and $\mathfrak{H}_{\mathbb{S}^{+}, \Sigma}^{\angle}$ defined in Section 2.3. In view of Remark 7.2, we denote, with a small abuse of notation, $\Pi_{\mathbb{S}_{0}^{+}, \Sigma}^{\top}:=\Pi_{\mathbb{S}^{+}, \Sigma}^{\top}+\pi_{0}$, so that $\Pi_{\mathbb{S}_{0}^{+}, \Sigma}^{\top}+\Pi_{\mathbb{S}^{+}, \Sigma}^{<}=$Id on the whole $L^{2} \times L^{2}$. We remind that $\mathbb{S}_{0}=\mathbb{S} \cup\{0\}$, where $\mathbb{S}$ is the set defined in (2.48). We denote by $\Pi_{\mathbb{S}_{0}}:=\Pi_{\mathbb{S}}^{\top}+\pi_{0}$, where $\Pi_{\mathbb{S}}^{\top}$ is defined below (2.58) together with the definition of $\Pi_{\mathbb{S}_{0}}^{\perp}$, so that we have $\Pi_{\mathbb{S}_{0}}+\Pi_{\mathbb{S}_{0}}^{\perp}=\mathrm{Id}$. 
Lemma 7.15. Let $M>0$. There is $\sigma_{M}>0$ (depending also on $\left.k_{0}, \tau, v\right)$ such that, assuming (7.14) with $\mu_{0} \geqq \sigma_{M}$, the following holds: the maps $\mathcal{W}_{1}, \mathcal{W}_{2}$ defined in (7.177) have the form

$$
\mathcal{W}_{i}=\widetilde{\mathcal{M}} \mathcal{C}+\mathcal{R}_{i}(\varepsilon),
$$

where, for any $i=1,2$, for all $s_{0} \leqq s \leqq S$,

$$
\left\|\mathcal{R}_{i}(\varepsilon) h\right\|_{S}^{k_{0}, v} \lesssim S, M \varepsilon v^{-1}\left(\|h\|_{S+\sigma_{M}}^{k_{0}, v}+\left\|\Im_{0}\right\|_{S+\sigma_{M}}^{k_{0}, v}\|h\|_{S_{0}+\sigma_{M}}^{k_{0}, v}\right)
$$

Moreover, for $\varepsilon v^{-1} \leqq \delta(S)$ small enough, the operators

$$
\mathcal{W}_{1}^{\perp}:=\Pi_{\mathbb{S}^{+}, \Sigma}^{\perp} \mathcal{W}_{1} \Pi_{\mathbb{S}_{0}}^{\perp}, \quad \mathcal{W}_{2}^{\perp}:=\Pi_{\mathbb{S}^{+}, \Sigma}^{\angle} \mathcal{W}_{2} \Pi_{\mathbb{S}_{0}}^{\perp},
$$

are invertible and, for all $s_{0} \leqq s \leqq S, i=1,2$,

$$
\begin{aligned}
\left\|\left(\mathcal{W}_{i}^{\perp}\right)^{ \pm 1} h\right\|_{S}^{k_{0}, v} & \lesssim S, M\|h\|_{s+\sigma_{M}}^{k_{0}, v}+\left\|\Im_{0}\right\|_{S+\sigma_{M}}^{k_{0}, v}\|h\|_{s_{0}+\sigma_{M}}^{k_{0}, v} \\
\left\|\Delta_{12}\left(\mathcal{W}_{i}^{\perp}\right)^{ \pm 1} h\right\|_{s_{1}} & \lesssim s_{1}, M \varepsilon v^{-1}\left\|i_{1}-i_{2}\right\|_{s_{1}+\sigma_{M}}\|h\|_{s_{1}+\sigma_{M}} .
\end{aligned}
$$

The operators $\mathcal{W}_{1}^{\perp}, \mathcal{W}_{2}^{\perp}$ map (anti)-reversible, respectively traveling, waves, into (anti)-reversible, respectively traveling, waves.

Proof. The formulae (7.178) and the estimates (7.179) follow by (7.177), Lemmata 3.12, 3.13, and (3.36), (7.23), (7.27), (7.91), (7.92), (7.112), (7.151), (7.152), (7.171). The invertibility of each $\mathcal{W}_{i}^{\perp}$ and the estimates (7.181) follow as in [2] and noting that $\Pi_{\mathbb{S}^{+}, \Sigma}^{<} \widetilde{\mathcal{M}} \mathcal{C} \Pi_{\mathbb{S}_{0}}^{\perp}=\Pi_{\mathbb{S}^{+}, \Sigma}^{<} \mathcal{M C} \Pi_{\mathbb{S}_{0}}^{\perp}$ are invertible on their ranges, with inverses $\left(\Pi_{\mathbb{S}^{+}, \Sigma}^{<} \mathcal{M C} \Pi_{\mathbb{S}_{0}}^{\perp}\right)^{-1}=\Pi_{\mathbb{S}_{0}}^{\perp}(\mathcal{M C})^{-1} \Pi_{\mathbb{S}^{+}, \Sigma}^{<}$. Since $\mathcal{Z}, \mathcal{E}, \mathcal{Q}, \widetilde{\mathcal{M}}, \boldsymbol{\Phi}_{M}, \boldsymbol{\Phi}$, $\mathcal{V}, \boldsymbol{\Psi}$ are reversibility and momentum preserving and using Remark 7.7 and Lemmata 3.23, 3.31, we deduce that $\mathcal{W}_{1}^{\perp}, \mathcal{W}_{2}^{\perp}$ map (anti)-reversible, respectively traveling, waves, into (anti)-reversible, respectively traveling, waves.

Remark 7.16. The time reparametrization $\mathcal{P}$ and the multiplication for the function $\rho$ (which is independent of the space variable), commute with the projections $\Pi_{\mathbb{S}^{+}, \Sigma}^{L}$ and $\Pi_{\mathbb{S}_{0}}^{\perp}$.

The operator $\mathcal{L}_{\omega}$ in (6.23) (that is (7.1)) is semi-conjugated to

$$
\mathcal{L}_{\perp}:=\left(\mathcal{W}_{2}^{\perp}\right)^{-1} \mathcal{L}_{\omega} \mathcal{W}_{1}^{\perp}=\Pi_{\mathbb{S}_{0}}^{\perp} \mathcal{L}_{9} \Pi_{\mathbb{S}_{0}}^{\perp}+\mathcal{R}^{f}
$$

where $\mathcal{R}^{f}$ is, by (7.180), (7.176), (7.178) (recall that $\widetilde{\mathcal{M}}$ is defined in (7.78)-(7.79)), and (2.59),

$$
\begin{aligned}
\mathcal{R}^{f}:= & \left(\mathcal{W}_{2}^{\perp}\right)^{-1} \Pi_{\mathbb{S}^{+}, \Sigma}^{<} \mathcal{R}_{2}(\varepsilon) \Pi_{\mathbb{S}_{0}} \mathcal{L}_{9} \Pi_{\mathbb{S}_{0}}^{\perp} \\
& -\left(\mathcal{W}_{2}^{\perp}\right)^{-1} \Pi_{\mathbb{S}^{+}, \Sigma}^{<} \mathcal{L} \Pi_{\mathbb{S}_{0}^{+}, \Sigma}^{\top} \mathcal{R}_{1}(\varepsilon) \Pi_{\mathbb{S}_{0}}^{\perp}-\varepsilon\left(\mathcal{W}_{2}^{\perp}\right)^{-1} \Pi_{\mathbb{S}^{+}, \Sigma}^{<} J R \mathcal{W}_{1}^{\perp}
\end{aligned}
$$


Lemma 7.17. The operator $\mathcal{R}^{f}$ in (7.184) has the finite rank form (7.4), (7.5). Moreover, let $\mathrm{q}_{0} \in \mathbb{N}_{0}$ and $M \geqq 2\left(k_{0}+\mathrm{q}_{0}\right)-\frac{3}{2}$. There exists $\aleph\left(M, \mathrm{q}_{0}\right)>0$ (depending also on $\left.k_{0}, \tau, v\right)$ such that, for any $n_{1}, n_{2} \in \mathbb{N}_{0}$, with $n_{1}+n_{2} \leqq$ $M-2\left(k_{0}+\mathrm{q}_{0}\right)+\frac{5}{2}$, and any $q \in \mathbb{N}_{0}^{v}$, with $|\mathrm{q}| \leqq \mathrm{q}_{0}$, the operator $\langle D\rangle^{n_{1}} \partial_{\varphi}^{\mathrm{q}} \mathcal{R}^{f}\langle D\rangle^{n_{2}}$ is $\mathcal{D}^{k_{0}}$-tame, with a tame constant satisfying

$$
\begin{aligned}
& \mathfrak{M}_{\langle D\rangle^{n_{1}} \partial_{\varphi}^{\mathrm{q}} \mathcal{R}^{f}\langle D\rangle^{n_{2}}}(s) \lesssim S, M, \mathrm{q}_{0} \varepsilon v^{-1}\left(1+\left\|\Im_{0}\right\|_{s+\aleph\left(M, \mathrm{q}_{0}\right)}^{k_{0}, v}\right), \quad \forall s_{0} \leqq s \leqq S, \\
& \left\|\langle D\rangle^{n_{1}} \partial_{\varphi}^{\mathrm{q}} \Delta_{12} \mathcal{R}^{f}\langle D\rangle^{n_{2}}\right\|_{\mathcal{L}\left(H^{s_{1}}\right)} \lesssim_{s_{1}, M, \mathrm{q}_{0}} \varepsilon v^{-1}\left\|i_{1}-i_{2}\right\|_{s_{1}+\aleph\left(M, \mathrm{q}_{0}\right)},
\end{aligned}
$$

for any $s_{1}$ as in (7.15).

Proof. The first two terms in (7.184) have the finite rank form (7.4) because of the presence of the finite dimensional projector $\Pi_{\mathbb{S}_{0}}$, respectively $\Pi_{\mathbb{S}_{0}^{+}, \Sigma}^{\top}$. In the last term, the operator $R$ has the finite rank form (7.4). The estimate (7.185) follows by (7.184), (7.177), (7.180), (7.168), (7.4), (3.7) and (7.179), (7.181), (7.169), (7.170), (7.5). The estimate (7.186) follows similarly.

Proposition 7.18. (Reduction of $\mathcal{L}_{\omega}$ up to smoothing operators) For all $(\omega, \kappa) \in$ $\mathrm{DC}(v, \tau) \times\left[\kappa_{1}, \kappa_{2}\right]$, the operator $\mathcal{L}_{\omega}$ in $(6.23)$ (that is $\left.(7.1)\right)$ is semi-conjugated via (7.180) to the real, reversible and momentum preserving operator $\mathcal{L}_{\perp}$ in (7.183). For all $(\omega, \kappa) \in \mathbb{R}^{v} \times\left[\kappa_{1}, \kappa_{2}\right]$, the extended operator defined by the right hand side in (7.183) has the form

$$
\mathcal{L}_{\perp}=\omega \cdot \partial_{\varphi} \mathbb{1}_{\perp}+\mathrm{i} \mathbf{D}_{\perp}+\mathbf{R}_{\perp}
$$

where $\mathbb{1}_{\perp}$ denotes the identity map of $\mathbf{H}_{\mathbb{S}_{0}}^{\perp}(c f r$. (2.58)) and

1. $\mathbf{D}_{\perp}$ is the diagonal operator

$$
\mathbf{D}_{\perp}:=\left(\begin{array}{cc}
\mathcal{D}_{\perp} & 0 \\
0 & -\overline{\mathcal{D}_{\perp}}
\end{array}\right), \quad \mathcal{D}_{\perp}:=\operatorname{diag}_{j \in \mathbb{S}_{0}^{c}} \mu_{j}, \quad \mathbb{S}_{0}^{c}:=\mathbb{Z} \backslash(\mathbb{S} \cup\{0\}),
$$

with eigenvalues $\mu_{j}:=\mathrm{m}_{\frac{3}{2}} \Omega_{j}(\kappa)+\mathrm{m}_{1} j+\mathrm{m}_{\frac{1}{2}}|j|^{\frac{1}{2}} \in \mathbb{R}$, where the real constants $\mathrm{m}_{\frac{3}{2}}, \mathrm{~m}_{1}, \mathrm{~m}_{\frac{1}{2}}$, defined respectively in (7.88), (7.144), (7.140), satisfy

$$
\left|\mathrm{m}_{\frac{3}{2}}-1\right|^{k_{0}, v}+\left|\mathrm{m}_{1}\right|^{k_{0}, v}+\left|\mathrm{m}_{\frac{1}{2}}\right|^{k_{0}, v} \lesssim \varepsilon ;
$$

in addition, for some $\sigma>0$,

$$
\left|\Delta_{12} \mathrm{~m}_{\frac{3}{2}}\right|+\left|\Delta_{12} \mathrm{~m}_{1}\right|+\left|\Delta_{12} \mathrm{~m}_{\frac{1}{2}}\right| \lesssim \varepsilon\left\|i_{1}-i_{2}\right\|_{s_{0}+\sigma} .
$$

2. The operator $\mathbf{R}_{\perp}$ is real, reversible and momentum preserving. Moreover, for any $\mathrm{q}_{0} \in \mathbb{N}_{0}, M>2\left(k_{0}+\mathrm{q}_{0}\right)-\frac{3}{2}$, there is a constant $\aleph\left(M, \mathrm{q}_{0}\right)>0$ (depending also on $\left.k_{0}, \tau, v\right)$ such that, assuming (7.14) with $\mu_{0} \geqq \aleph\left(M, q_{0}\right)$, for any $s_{0} \leqq s \leqq S, \mathrm{q} \in \mathbb{N}_{0}^{v}$, with $|\mathrm{q}| \leqq \mathrm{q}_{0}$, the operators $\partial_{\varphi}^{\bar{q}} \mathbf{R}_{\perp},\left[\partial_{\varphi}^{\mathrm{q}} \mathbf{R}_{\perp}, \partial_{x}\right]$ are $\mathcal{D}^{k_{0}}$-tame with tame constants satisfying

$$
\mathfrak{M}_{\partial_{\varphi}^{q} \mathbf{R}_{\perp}}(s), \mathfrak{M}_{\left[\partial_{\varphi}^{\mathrm{q}} \mathbf{R}_{\perp}, \partial_{x}\right]}(s) \lesssim s, M, \mathrm{q}_{0} \varepsilon v^{-1}\left(1+\left\|\mathfrak{I}_{0}\right\|_{s+\aleph\left(M, \mathrm{q}_{0}\right)}^{k_{0}, v}\right) .
$$


Moreover, for any $\mathrm{q} \in \mathbb{N}_{0}^{v}$, with $|\mathrm{q}| \leqq \mathrm{q}_{0}$,

$$
\left\|\partial_{\varphi}^{\mathrm{q}} \Delta_{12} \mathbf{R}_{\perp}\right\|_{\mathcal{L}\left(H^{s_{0}}\right)}+\left\|\partial_{\varphi}^{\mathrm{q}} \Delta_{12}\left[\mathbf{R}_{\perp}, \partial_{x}\right]\right\|_{\mathcal{L}\left(H^{s_{0}}\right)} \lesssim_{M} \varepsilon v^{-1}\left\|i_{1}-i_{2}\right\|_{s_{0}+\aleph\left(M, \mathrm{q}_{0}\right)} .
$$

Proof. By (7.183) and (7.168) we deduce (7.187) with

$$
\mathbf{R}_{\perp}:=\Pi_{\mathbb{S}_{0}}^{\perp}\left(\mathbf{R}_{9}^{(0, d)}+\mathbf{T}_{9, M}\right) \Pi_{\mathbb{S}_{0}}^{\perp}+\mathcal{R}^{f} .
$$

The estimates (7.188)-(7.189) follow by Lemmata 7.9, 7.12, 7.13. The estimate (7.190) follows by Lemmata 3.6, 3.13, (7.169) and (7.170), (7.185), choosing $\left(n_{1}, n_{2}\right)=(1,0),(0,1)$. The estimate $(7.191)$ follows similarly. The operator $\mathcal{L}_{\omega}$ in (6.23) is reversible and momentum preserving (Lemma 7.1). By Sections 7.2-7.6, the maps $\mathcal{Z}, \mathcal{E}, \mathcal{Q}, \widetilde{\mathcal{M}}, \boldsymbol{\Phi}_{M}, \boldsymbol{\Phi}, \mathcal{V}, \boldsymbol{\Psi}$ are reversibility and momentum preserving. Therefore, using also (7.18), (7.24) and Lemmata 3.23 and 3.31, we deduce that the operator $\mathcal{L}_{\perp}$ in (7.183) is reversible and momentum preserving. Since i $\mathbf{D}_{\perp}$ is reversible and momentum preserving, we deduce that $\mathbf{R}_{\perp}$ is reversible and momentum preserving.

\section{Almost-Diagonalization and Invertibility of $\mathcal{L}_{\omega}$}

In Proposition 7.18 we obtained the operator $\mathcal{L}_{\perp}$ in (7.187) which is diagonal and constant coefficient up to the bounded operator $\mathbf{R}_{\perp}(\varphi)$. In this section we complete the diagonalization of $\mathcal{L}_{\perp}$ implementing a KAM iterative scheme. As starting point, we consider the real, reversible and momentum preserving operator, acting in $\mathbf{H}_{\mathbb{S}_{0}}^{\perp}$,

$$
\mathbf{L}_{0}:=\mathbf{L}_{0}(i):=\mathcal{L}_{\perp}=\omega \cdot \partial_{\varphi} \mathbb{1}_{\perp}+\mathrm{i} \mathbf{D}_{0}+\mathbf{R}_{\perp}^{(0)}
$$

defined for all $(\omega, \kappa) \in \mathbb{R}^{v} \times\left[\kappa_{1}, \kappa_{2}\right]$, with diagonal part (with respect to the exponential basis)

$$
\mathbf{D}_{0}:=\left(\begin{array}{cc}
\mathcal{D}_{0} & 0 \\
0 & -\overline{\mathcal{D}_{0}}
\end{array}\right), \mathcal{D}_{0}:=\operatorname{diag}_{j \in \mathbb{S}_{0}^{c}} \mu_{j}^{(0)}, \mu_{j}^{(0)}:=\mathrm{m}_{\frac{3}{2}} \Omega_{j}(\kappa)+\mathrm{m}_{1} j+\mathrm{m}_{\frac{1}{2}}|j|^{\frac{1}{2}},
$$

where $\mathbb{S}_{0}^{c}=\mathbb{Z} \backslash \mathbb{S}_{0}, \mathbb{S}_{0}=\mathbb{S} \cup\{0\}$, the real constants $\mathrm{m}_{\frac{3}{2}}, \mathrm{~m}_{1}, \mathrm{~m}_{\frac{1}{2}}$ satisfy (7.188)(7.189) and

$$
\mathbf{R}_{\perp}^{(0)}:=\mathbf{R}_{\perp}:=\left(\frac{R_{\perp}^{(0, d)}}{R_{\perp}^{(0, o)}} \frac{R_{\perp}^{(0, o)}}{R_{\perp}^{(0, d)}}\right), R_{\perp}^{(0, d)}: H_{\mathbb{S}_{0}}^{\perp} \rightarrow H_{\mathbb{S}_{0}}^{\perp}, \quad R_{\perp}^{(0, o)}: H_{-\mathbb{S}_{0}}^{\perp} \rightarrow H_{\mathbb{S}_{0}}^{\perp},
$$

which is a real, reversible, momentum preserving operator satisfying (7.190), (7.191). We denote $H_{ \pm \mathbb{S}_{0}}^{\perp}=\left\{h(x)=\sum_{j \notin \pm \mathbb{S}_{0}} h_{j} e^{ \pm \mathrm{i} j x} \in L^{2}\right\}$. Note that

$$
\overline{\mathcal{D}_{0}}: H_{-\mathbb{S}_{0}}^{\perp} \rightarrow H_{-\mathbb{S}_{0}}^{\perp}, \quad \overline{\mathcal{D}_{0}}=\operatorname{diag}_{j \in-\mathbb{S}_{0}^{c}}\left(\mu_{-j}^{(0)}\right) .
$$


Proposition 7.18 implies that the operator $\mathbf{R}_{\perp}^{(0)}$ satisfies the tame estimates of Lemma 8.1 below by fixing the constant $M$ large enough (which means performing sufficiently many regularizing steps in Section 7.4), namely

$$
M:=\left[2\left(k_{0}+s_{0}+\mathrm{b}\right)-\frac{3}{2}\right]+1 \in \mathbb{N},
$$

where

$$
\mathrm{b}:=[\mathrm{a}]+2 \in \mathbb{N}, \quad \mathrm{a}:=3 \tau_{1} \geqq 1, \quad \tau_{1}:=k_{0}+\left(k_{0}+1\right) \tau .
$$

These conditions imply the convergence of the iterative scheme (8.46)-(8.47), see Lemma 8.8. We also set

$$
\mu(\mathrm{b}):=\aleph\left(M, s_{0}+\mathrm{b}\right),
$$

where the constant $\aleph\left(M, q_{0}\right)$ is given in Proposition 7.18.

Lemma 8.1. (Smallness of $\mathbf{R}_{\perp}^{(0)}$ ) Assume (7.14) with $\mu_{0} \geqq \mu(\mathrm{b})$. Then the operators $\mathbf{R}_{\perp}^{(0)},\left[\mathbf{R}_{\perp}^{(0)}, \partial_{x}\right]$, and $\partial_{\varphi_{m}}^{s_{0}} \mathbf{R}_{\perp}^{(0)},\left[\partial_{\varphi_{m}}^{s_{0}} \mathbf{R}_{\perp}^{(0)}, \partial_{x}\right], \partial_{\varphi_{m}}^{s_{0}+\mathrm{b}} \mathbf{R}_{\perp}^{(0)},\left[\partial_{\varphi_{m}}^{s_{0}+\mathrm{b}} \mathbf{R}_{\perp}^{(0)}, \partial_{x}\right]$, $m=1, \ldots, v$, are $\mathcal{D}^{k_{0}}$-tame and, defining

$$
\begin{aligned}
\mathbb{M}_{0}(s):= & \max \left\{\mathfrak{M}_{\mathbf{R}_{\perp}^{(0)}}(s), \mathfrak{M}_{\left[\mathbf{R}_{\perp}^{(0)}, \partial_{x}\right]}(s),\right. \\
& \mathfrak{M}_{\left.\partial_{\varphi_{m}}^{s_{0}} \mathbf{R}_{\perp}^{(0)}(s), \mathfrak{M}_{\left[\partial_{\varphi_{m}}^{s_{0}} \mathbf{R}_{\perp}^{(0)}, \partial_{x}\right]}(s), m=1, \ldots, v\right\},} \\
\mathbb{M}_{0}(s, \mathrm{~b}):= & \max \left\{\mathfrak{M}_{\partial_{\varphi_{m}}^{s_{0}+\mathrm{b}} \mathbf{R}_{\perp}^{(0)}}(s), \mathfrak{M}_{\left[\partial_{\varphi_{m}}^{s_{0}+\mathrm{b}} \mathbf{R}_{\perp}^{(0)}, \partial_{x}\right]}(s), m=1, \ldots, v\right\},
\end{aligned}
$$

we have, for all $s_{0} \leqq s \leqq S$,

$$
\begin{aligned}
& \mathfrak{M}_{0}(s, \mathrm{~b}):=\max \left\{\mathbb{M}_{0}(s), \mathbb{M}_{0}(s, \mathrm{~b})\right\} \leqq C(S) \frac{\varepsilon}{v}\left(1+\left\|\mathfrak{I}_{0}\right\|_{s+\mu(\mathrm{b})}^{k_{0}, v}\right), \\
& \mathfrak{M}_{0}\left(s_{0}, \mathrm{~b}\right) \leqq C(S) \frac{\varepsilon}{v}
\end{aligned}
$$

Moreover, for all $\mathrm{q} \in \mathbb{N}_{0}^{v}$, with $|\mathrm{q}| \leqq s_{0}+\mathrm{b}$,

$$
\left\|\partial_{\varphi}^{q} \Delta_{12} \mathbf{R}_{\perp}^{(0)}\right\|_{\mathcal{L}\left(H^{s} 0\right)},\left\|\Delta_{12}\left[\partial_{\varphi}^{q} \mathbf{R}_{\perp}^{(0)}, \partial_{x}\right]\right\|_{\mathcal{L}\left(H^{s} 0\right)} \leqq C(S) \varepsilon v^{-1}\left\|i_{1}-i_{2}\right\|_{s_{0}+\mu(\mathrm{b})} .
$$

Proof. Recalling (8.8), (8.9), the bounds (8.10)-(8.11) follow by (7.190), (8.5), (8.7), (7.191).

We perform the almost-reducibility of $\mathbf{L}_{0}$ along the scale

$$
N_{-1}:=1, \quad N_{\mathrm{n}}:=N_{0}^{\chi^{\mathrm{n}}}, \quad \forall \mathrm{n} \in \mathbb{N}_{0}, \quad \chi:=3 / 2 .
$$

Theorem 8.2. (Almost-diagonalization of $\mathbf{L}_{0}$ : KAM iteration) There exists $\tau_{2}(\tau, v)>$ $\tau_{1}(\tau, v)+$ a (with $\tau_{1}$, a defined in (8.6)) such that, for all $S>s_{0}$, there is $N_{0}:=N_{0}(S, \mathrm{~b}) \in \mathbb{N}$ such that, if

$$
N_{0}^{\tau_{2}} \mathfrak{M}_{0}\left(s_{0}, \mathrm{~b}\right) v^{-1} \leqq 1
$$

then, for all $\overline{\mathrm{n}} \in \mathbb{N}_{0}, \mathrm{n}=0,1, \ldots, \overline{\mathrm{n}}$ : 
(S1 $)_{\mathrm{n}}$ There exists a real, reversible and momentum preserving operator

$$
\begin{aligned}
& \mathbf{L}_{\mathrm{n}}:=\omega \cdot \partial_{\varphi} \mathbb{1}_{\perp}+\mathrm{i} \mathbf{D}_{\mathrm{n}}+\mathbf{R}_{\perp}^{(\mathrm{n})}, \\
& \mathbf{D}_{\mathrm{n}}:=\left(\begin{array}{cc}
\mathcal{D}_{\mathrm{n}} & 0 \\
0 & -\overline{\mathcal{D}_{\mathrm{n}}}
\end{array}\right), \quad \mathcal{D}_{\mathrm{n}}:=\operatorname{diag}_{j \in \mathbb{S}_{0}^{c}} \mu_{j}^{(\mathrm{n})},
\end{aligned}
$$

defined for all $(\omega, \kappa)$ in $\mathbb{R}^{v} \times\left[\kappa_{1}, \kappa_{2}\right]$, where $\mu_{j}^{(\mathrm{n})}$ are $k_{0}$-times differentiable real functions

$$
\mu_{j}^{(\mathrm{n})}(\omega, \kappa):=\mu_{j}^{(0)}(\omega, \kappa)+\mathfrak{r}_{j}^{(\mathrm{n})}(\omega, \kappa), \quad \mu_{j}^{(0)}=\mathrm{m}_{\frac{3}{2}} \Omega_{j}(\kappa)+\mathrm{m}_{1} j+\mathrm{m}_{\frac{1}{2}}|j|^{\frac{1}{2}},
$$

satisfying $\mathfrak{r}_{j}^{(0)}=0$ and, for $\mathrm{n} \geqq 1$,

$$
\left|\mathfrak{r}_{j}^{(\mathrm{n})}\right|^{k_{0}, v} \leqq C(S, \mathrm{~b}) \varepsilon v^{-1},\left|\mu_{j}^{(\mathrm{n})}-\mu_{j}^{(\mathrm{n}-1)}\right|^{k_{0}, v} \leqq C(S, \mathrm{~b}) \varepsilon v^{-1} N_{\mathrm{n}-2}^{-\mathrm{a}}, \quad \forall j \in \mathbb{S}_{0}^{c} .
$$

The remainder

$$
\mathbf{R}_{\perp}^{(\mathrm{n})}:=\left(\frac{R_{\perp}^{(\mathrm{n}, d)}}{R_{\perp}^{(\mathrm{n}, o)}} \frac{R_{\perp}^{(\mathrm{n}, o)}}{R_{\perp}^{(\mathrm{n}, d)}}\right), \quad R_{\perp}^{(\mathrm{n}, d)}: H_{\mathbb{S}_{0}}^{\perp} \rightarrow H_{\mathbb{S}_{0}}^{\perp}, R_{\perp}^{(\mathrm{n}, o)}: H_{-\mathbb{S}_{0}}^{\perp} \rightarrow H_{\mathbb{S}_{0}}^{\perp}
$$

is $\mathcal{D}^{k_{0}}$-modulo-tame: more precisely, the operators $R_{\perp}^{(\mathrm{n}, d)}, R_{\perp}^{(\mathrm{n}, o)},\left\langle\partial_{\varphi}\right\rangle^{\mathrm{b}} R_{\perp}^{(\mathrm{n}, d)}$, $\left\langle\partial_{\varphi}\right\rangle^{\mathrm{b}} R_{\perp}^{(\mathrm{n}, o)}$, are $\mathcal{D}^{k_{0}}$-modulo-tame with modulo-tame constants

$$
\begin{aligned}
& \mathfrak{M}_{\mathrm{n}}^{\sharp}(s):=\mathfrak{M}_{\mathbf{R}_{\perp}^{(\mathrm{n})}}^{\sharp}(s):=\max \left\{\mathfrak{M}_{R_{\perp}^{(\mathrm{n}, d)}}^{\sharp}(s), \mathfrak{M}_{R_{\perp}^{(\mathrm{n}, o)}}^{\sharp}(s)\right\}, \\
& \mathfrak{M}_{\mathrm{n}}^{\sharp}(s, \mathrm{~b}):=\mathfrak{M}_{\left\langle\partial_{\varphi}\right\rangle^{\mathrm{b}} \mathbf{R}_{\perp}^{(\mathrm{n})}}^{\sharp}(s):=\max \left\{\mathfrak{M}_{\left\langle\partial_{\varphi}\right\rangle^{\mathrm{b}} R_{\perp}^{(\mathrm{n}, d)}}^{\sharp}(s), \mathfrak{M}_{\left\langle\partial_{\varphi}\right\rangle^{\mathrm{b}} R_{\perp}^{(\mathrm{n}, o)}}^{\sharp}(s)\right\},
\end{aligned}
$$

which satisfy, for some constant $C_{*}\left(s_{0}, \mathrm{~b}\right)>0$, for all $s_{0} \leqq s \leqq S$,

$$
\mathfrak{M}_{\mathrm{n}}^{\sharp}(s) \leqq C_{*}\left(s_{0}, \mathrm{~b}\right) \mathfrak{M}_{0}(s, \mathrm{~b}) N_{\mathrm{n}-1}^{-\mathrm{a}}, \quad \mathfrak{M}_{\mathrm{n}}^{\sharp}(s, \mathrm{~b}) \leqq C_{*}\left(s_{0}, \mathrm{~b}\right) \mathfrak{M}_{0}(s, \mathrm{~b}) N_{\mathrm{n}-1} .
$$

Define the sets $\Lambda_{\mathrm{n}}^{v}=\Lambda_{\mathrm{n}}^{v}(i)$ by $\Lambda_{0}^{v}:=\mathrm{DC}(2 v, \tau) \times\left[\kappa_{1}, \kappa_{2}\right]$ and, for $\mathrm{n} \geqq 1$,

$$
\begin{aligned}
\Lambda_{\mathrm{n}}^{v}:= & \left\{\lambda=(\omega, \kappa) \in \Lambda_{\mathrm{n}-1}^{v}:\right. \\
& \left|\omega \cdot \ell+\mu_{j}^{(\mathrm{n}-1)}-\mu_{j^{\prime}}^{(\mathrm{n}-1)}\right| \geqq v\left\langle\left.\left|j^{\frac{3}{2}}-\right| j^{\prime}\right|^{\frac{3}{2}}\right\rangle\langle\ell\rangle^{-\tau} \\
& \forall|\ell| \leqq N_{\mathrm{n}-1}, j, j^{\prime} \notin \mathbb{S}_{0},\left(\ell, j, j^{\prime}\right) \neq(0, j, j), \text { with } \vec{j} \cdot \ell+j-j^{\prime}=0, \\
& \left|\omega \cdot \ell+\mu_{j}^{(\mathrm{n}-1)}+\mu_{j^{\prime}}^{(\mathrm{n}-1)}\right| \geqq v\left(|j|^{\frac{3}{2}}+\left|j^{\prime}\right|^{\frac{3}{2}}\right)\langle\ell\rangle-\tau \\
& \left.\forall|\ell| \leqq N_{\mathrm{n}-1}, j, j^{\prime} \notin \mathbb{S}_{0} \text { with } \vec{j} \cdot \ell+j+j^{\prime}=0\right\} .
\end{aligned}
$$

For $\mathrm{n} \geqq 1$ there exists a real, reversibility and momentum preserving map, defined for all $(\omega, \kappa) \in \mathbb{R}^{v} \times\left[\kappa_{1}, \kappa_{2}\right]$, of the form

$$
\boldsymbol{\Phi}_{\mathrm{n}-1}=e^{\mathbf{X}_{\mathrm{n}-1}}, \mathbf{X}_{\mathrm{n}-1}:=\left(\frac{X_{\mathrm{n}-1}^{(d)}}{X_{\mathrm{n}-1}^{(o)}} \frac{X_{\mathrm{n}-1}^{(o)}}{X_{\mathrm{n}-1}^{(d)}}\right), X_{\mathrm{n}-1}^{(d)}: H_{\mathbb{S}_{0}}^{\perp} \rightarrow H_{\mathbb{S}_{0}}^{\perp}, X_{\mathrm{n}-1}^{(o)}: H_{-\mathbb{S}_{0}}^{\perp} \rightarrow H_{\mathbb{S}_{0}}^{\perp},
$$


such that, for all $\lambda \in \Lambda_{\mathrm{n}}^{v}$, the following conjugation formula holds:

$$
\mathbf{L}_{\mathrm{n}}=\boldsymbol{\Phi}_{\mathrm{n}-1}^{-1} \mathbf{L}_{\mathrm{n}-1} \boldsymbol{\Phi}_{\mathrm{n}-1} \text {. }
$$

The operators $\mathbf{X}_{\mathrm{n}-1},\left\langle\partial_{\varphi}\right\rangle^{\mathrm{b}} \mathbf{X}_{\mathrm{n}-1}$, are $\mathcal{D}^{k_{0}}$-modulo-tame with modulo tame constants satisfying, for all $s_{0} \leqq s \leqq S$,

$$
\begin{aligned}
\mathfrak{M}_{\mathbf{X}_{\mathrm{n}-1}}^{\sharp}(s) & \leqq C\left(s_{0}, \mathrm{~b}\right) v^{-1} N_{\mathrm{n}-1}^{\tau_{1}} N_{\mathrm{n}-2}^{-\mathrm{a}} \mathfrak{M}_{0}(s, \mathrm{~b}), \\
\mathfrak{M}_{\left\langle\partial_{\varphi}\right\rangle^{\mathrm{b}} \mathbf{X}_{\mathrm{n}-1}}^{\#}(s) & \leqq C\left(s_{0}, \mathrm{~b}\right) v^{-1} N_{\mathrm{n}-1}^{\tau_{1}} N_{\mathrm{n}-2} \mathfrak{M}_{0}(s, \mathrm{~b}) .
\end{aligned}
$$

(S2) $)_{\mathrm{n}}$ Let $i_{1}(\omega, \kappa), i_{2}(\omega, \kappa)$ such that $\mathbf{R}_{\perp}^{(\mathrm{n})}\left(i_{1}\right), \mathbf{R}_{\perp}^{(\mathrm{n})}\left(i_{2}\right)$ satisfy (8.10), (8.11). Then, for all $(\omega, \kappa) \in \Lambda_{\mathrm{n}}^{v_{1}}\left(i_{1}\right) \cap \Lambda_{\mathrm{n}}^{v_{2}}\left(i_{2}\right)$ with $v_{1}, v_{2} \in[v / 2,2 v]$,

$$
\begin{aligned}
& \left\|\left|\Delta_{12} \mathbf{R}_{\perp}^{(\mathrm{n})}\right|\right\|_{\mathcal{L}_{\left(H^{s}\right)}} \lesssim_{S, \mathrm{~b}} \varepsilon v^{-1} N_{\mathrm{n}-1}^{-\mathrm{a}}\left\|i_{1}-i_{2}\right\|_{S_{0}+\mu(\mathrm{b})}, \\
& \left\|\left|\left\langle\partial_{\varphi}\right\rangle^{\mathrm{b}} \Delta_{12} \mathbf{R}_{\perp}^{(\mathrm{n})}\right|\right\|_{\mathcal{L}\left(H^{\left.s_{0}\right)}\right.} \lesssim_{S, \mathrm{~b}} \varepsilon v^{-1} N_{\mathrm{n}-1}\left\|i_{1}-i_{2}\right\|_{s_{0}+\mu(\mathrm{b})} .
\end{aligned}
$$

Furthermore, for $\mathrm{n} \geqq 1$, for all $j \in \mathbb{S}_{0}^{c}$,

$$
\begin{aligned}
& \left|\Delta_{12}\left(\mathfrak{r}_{j}^{(\mathrm{n})}-\mathfrak{r}_{j}^{(\mathrm{n}-1)}\right)\right| \leqq C\left\|\left|\Delta_{12} \mathbf{R}_{\perp}^{(\mathrm{n})}\right|\right\|_{\mathcal{L}\left(H^{s_{0}}\right)}, \\
& \left|\Delta_{12} \mathfrak{r}_{j}^{(\mathrm{n})}\right| \leqq C(S, \mathrm{~b}) \varepsilon v^{-1}\left\|i_{1}-i_{2}\right\|_{s_{0}+\mu(\mathrm{b})} .
\end{aligned}
$$

$(\mathbf{S 3})_{\mathrm{n}}$ Let $i_{1}, i_{2}$ be like in $(\mathbf{S 2})_{\mathrm{n}}$ and $0<\rho<v / 2$. Then

$$
\varepsilon v^{-1} C(S) N_{\mathrm{n}-1}^{\tau+1}\left\|i_{1}-i_{2}\right\|_{s_{0}+\mu(\mathrm{b})} \leqq \rho \Rightarrow \Lambda_{\mathrm{n}}^{v}\left(i_{1}\right) \subseteq \Lambda_{\mathrm{n}}^{v-\rho}\left(i_{2}\right) .
$$

Theorem 8.2 implies also that the invertible operator

$$
\mathbf{U}_{\overline{\mathrm{n}}}:=\boldsymbol{\Phi}_{0} \circ \ldots \circ \boldsymbol{\Phi}_{\overline{\mathrm{n}}-1}, \quad \overline{\mathrm{n}} \geqq 1,
$$

has almost diagonalized $\mathbf{L}_{0}$. We have indeed the following corollary.

Theorem 8.3. (Almost-diagonalization of $\mathbf{L}_{0}$ ) Assume (7.14) with $\mu_{0} \geqq \mu(\mathrm{b})$. For all $S>s_{0}$, there exist $N_{0}=N_{0}(S, \mathrm{~b})>0$ and $\delta_{0}=\delta_{0}(S)>0$ such that, if the smallness condition

$$
N_{0}^{\tau_{2}} \varepsilon v^{-2} \leqq \delta_{0}
$$

holds, where $\tau_{2}=\tau_{2}(\tau, v)$ is defined in Theorem 8.2, then, for all $\overline{\mathrm{n}} \in \mathbb{N}$ and for all $(\omega, \kappa) \in \mathbb{R}^{v} \times\left[\kappa_{1}, \kappa_{2}\right]$ the operator $\mathbf{U}_{\overline{\mathrm{n}}}$ in (8.28) is well-defined, the operators $\mathbf{U}_{\overline{\mathrm{n}}}^{ \pm 1}-\mathbb{1}_{\perp}$ are $\mathcal{D}^{k_{0}}$-modulo-tame with modulo-tame constants satisfying, for all $s_{0} \leqq s \leqq S$,

$$
\mathfrak{M}_{\mathbf{U}_{\overline{\mathrm{n}}}^{ \pm 1}-\mathbb{1}_{\perp}}^{\sharp}(s) \lesssim s \varepsilon v^{-2} N_{0}^{\tau_{1}}\left(1+\left\|\mathfrak{I}_{0}\right\|_{s+\mu(\mathrm{b})}^{k_{0}, v}\right),
$$

where $\tau_{1}$ is given by (8.6). Moreover $\mathbf{U}_{\overline{\mathrm{n}}}, \mathbf{U}_{\overline{\mathrm{n}}}^{-1}$ are real, reversibility and momentum preserving. The operator $\mathbf{L}_{\overline{\mathrm{n}}}=\omega \cdot \partial_{\varphi} \mathbb{1}_{\perp}+\mathrm{i} \mathbf{D}_{\overline{\mathrm{n}}}+\mathbf{R}_{\perp}^{(\overline{\mathrm{n}})}$, defined in (8.14) with $\mathrm{n}=\overline{\mathrm{n}}$ is real, reversible and momentum preserving. The operator $\mathbf{R}_{\perp}^{(\overline{\mathrm{n}})}$ is $\mathcal{D}^{k_{0}}$ modulo-tame with a modulo-tame constant satisfying, for all $s_{0} \leqq s \leqq S$,

$$
\mathfrak{M}_{\mathbf{R}_{\perp}^{(\overline{\mathrm{n}})}}^{\sharp}(s) \lesssim S \varepsilon v^{-1} N_{\overline{\mathrm{n}}-1}^{-\mathrm{a}}\left(1+\left\|\Im_{0}\right\|_{s+\mu(\mathrm{b})}^{k_{0}, v}\right) .
$$

Moreover, for all $(\omega, \kappa)$ in $\Lambda_{\overline{\mathrm{n}}}^{v}=\Lambda_{\overline{\mathrm{n}}}^{v}(i)=\bigcap_{\mathrm{n}=0}^{\overline{\mathrm{n}}} \Lambda_{\mathrm{n}}^{v}$, where the sets $\Lambda_{\mathrm{n}}^{v}$ are defined in (8.20), the conjugation formula $\mathbf{L}_{\overline{\mathrm{n}}}:=\mathbf{U}_{\overline{\mathrm{n}}}^{-1} \mathbf{L}_{0} \mathbf{U}_{\overline{\mathrm{n}}}$ holds. 


\section{Proof of Theorem 8.2}

The proof of Theorem 8.2 is inductive. We first show that $(\mathbf{S 1})_{\mathrm{n}}-(\mathbf{S 3})_{\mathrm{n}}$ hold when $\mathrm{n}=0$.

The step $\mathrm{n}=0$. Proof of $(\mathbf{S 1})_{0}$. Properties (8.14)-(8.15), (8.17) for $\mathrm{n}=0$ hold by (8.1), (8.2), (8.3) with $\mathfrak{r}_{j}^{(0)}=0$. We now prove that also (8.19) for $\mathrm{n}=0$ holds.

Lemma 8.4. We have $\mathfrak{M}_{0}^{\sharp}(s), \mathfrak{M}_{0}^{\sharp}(s, \mathrm{~b}) \lesssim s_{0}, \mathrm{~b} \mathfrak{M}_{0}(s, \mathrm{~b})$.

Proof. Let $R \in\left\{R_{\perp}^{(0, d)}, R_{\perp}^{(0, o)}\right\}$. We prove that $\left\langle\partial_{\varphi}\right\rangle^{\mathrm{b}} R$ is $\mathcal{D}^{k_{0}}$-modulo-tame. Using the inequality

$$
\left\langle\ell-\ell^{\prime}\right\rangle^{2 \mathrm{q}_{0}}\left\langle j-j^{\prime}\right\rangle^{2} \lesssim \mathrm{q}_{0} 1+\left|\ell-\ell^{\prime}\right|^{2 \mathrm{q}_{0}}+\left|j-j^{\prime}\right|^{2}+\left|\ell-\ell^{\prime}\right|^{2 \mathrm{q}_{0}}\left|j-j^{\prime}\right|^{2},
$$

it follows, recalling (3.35), (8.10), (the matrix elements of the commutator $\left[\partial_{x}, A\right]$ are $\left.\mathrm{i}\left(j-j^{\prime}\right) A_{j}^{j^{\prime}}\left(\ell-\ell^{\prime}\right)\right)$, that, for any $j^{\prime} \in \mathbb{S}_{0}^{c}, \ell^{\prime} \in \mathbb{Z}^{v}$,

$$
\begin{array}{r}
v^{2|k|} \sum_{\ell, j}\langle\ell, j\rangle^{2 s}\left\langle\ell-\ell^{\prime}\right\rangle^{2\left(s_{0}+\mathrm{b}\right)}\left\langle j-j^{\prime}\right\rangle^{2}\left|\partial_{\lambda}^{k} R_{j}^{j^{\prime}}\left(\ell-\ell^{\prime}\right)\right|^{2} \\
\lesssim_{\mathrm{b}} \mathfrak{M}_{0}\left(s_{0}, \mathrm{~b}\right)^{2}\left\langle\ell^{\prime}, j^{\prime}\right\rangle^{2 s}+\mathfrak{M}_{0}(s, \mathrm{~b})^{2}\left\langle\ell^{\prime}, j^{\prime}\right\rangle^{2 s_{0}} .
\end{array}
$$

Let $s_{0} \leqq s \leqq S$. Then, for any $|k| \leqq k_{0}$, by Cauchy-Schwartz inequality, we have

$$
\begin{aligned}
& \left\|\left|\left\langle\partial_{\varphi}\right\rangle^{\mathrm{b}} \partial_{\lambda}^{k} R\right| h\right\|_{s}^{2} \leqq \sum_{\ell, j}\langle\ell, j\rangle^{2 s}\left(\sum_{\ell^{\prime}, j^{\prime}}\left\langle\ell-\ell^{\prime}\right\rangle^{\mathrm{b}}\left|\left(\partial_{\lambda}^{k} R\right)_{j}^{j^{\prime}}\left(\ell-\ell^{\prime}\right)\right|\left|h_{\ell^{\prime}, j^{\prime}}\right|\right)^{2} \\
& \quad \leqq \sum_{\ell, j}\langle\ell, j\rangle^{2 s}\left(\sum_{\ell^{\prime}, j^{\prime}}\left\langle\ell-\ell^{\prime}\right\rangle^{s_{0}+\mathrm{b}}\left\langle j-j^{\prime}\right\rangle\left|\left(\partial_{\lambda}^{k} R\right)_{j}^{j^{\prime}}\left(\ell-\ell^{\prime}\right)\right|\left|h_{\ell^{\prime}, j^{\prime}}\right| \frac{1}{\left\langle\ell-\ell^{\prime}\right\rangle^{s_{0}}\left\langle j-j^{\prime}\right\rangle}\right)^{2} \\
& \quad \lesssim s_{0} \sum_{\ell, j}\langle\ell, j\rangle^{2 s} \sum_{\ell^{\prime}, j^{\prime}}\left\langle\ell-\ell^{\prime}\right\rangle^{2\left(s_{0}+\mathrm{b}\right)}\left\langle j-j^{\prime}\right\rangle^{2}\left|\left(\partial_{\lambda} R\right)_{j}^{j^{\prime}}\left(\ell-\ell^{\prime}\right)\right|^{2}\left|h_{\ell^{\prime}, j^{\prime}}\right|^{2} \\
& \quad \sum_{s_{0}, \mathrm{~b}}^{(8.31)} v^{-2|k|} \sum_{\ell^{\prime}, j^{\prime}}\left|h_{\ell^{\prime}, j^{\prime}}\right|^{2}\left(\mathfrak{M}_{0}\left(s_{0}, \mathrm{~b}\right)^{2}\left\langle\ell^{\prime}, j^{\prime}\right\rangle^{2 s}+\mathfrak{M}_{0}(s, \mathrm{~b})^{2}\left\langle\ell^{\prime}, j^{\prime}\right\rangle^{2 s_{0}}\right) .
\end{aligned}
$$

Therefore, we obtain $\mathfrak{M}_{\left\langle\partial_{\varphi}\right\rangle^{\mathrm{b}} R}^{\sharp}(s) \lesssim_{s_{0}, \mathrm{~b}} \mathfrak{M}_{0}(s, \mathrm{~b})$ and then $\mathfrak{M}_{0}^{\sharp}(s, \mathrm{~b}) \lesssim_{s_{0}, \mathrm{~b}} \mathfrak{M}_{0}(s, \mathrm{~b})$. The inequality $\mathfrak{M}_{0}^{\sharp}(s) \lesssim_{s_{0}} \mathfrak{M}_{0}(s, \mathrm{~b})$ follows similarly.

Proof of $(\mathbf{S 2})_{0}$. The proof of estimates (8.23), (8.24) at $\mathrm{n}=0$ follows by (8.11), arguing similarly to Lemma 8.4.

Proof of $(\mathbf{S 3})_{0}$. It is trivial since, by definition, $\Lambda_{0}^{v}\left(i_{1}\right)=\operatorname{DC}(2 v, \tau) \times$ $\left[\kappa_{1}, \kappa_{2}\right] \subset \Lambda_{0}^{v-\rho}\left(i_{2}\right)$. 
The reducibility step. We now describe the generic inductive step, showing how to transform $\mathbf{L}_{n}$ into $\mathbf{L}_{n+1}$ by the conjugation with $\boldsymbol{\Phi}_{n}$. For sake of simplicity in the notation, we drop the index $n$ and we write + instead of $n+1$, so that we write $\mathbf{L}:=\mathbf{L}_{\mathrm{n}}, \mathbf{L}_{+}:=\mathbf{L}_{\mathrm{n}+1}, \mathbf{R}_{\perp}:=\mathbf{R}_{\perp}^{(\mathrm{n})}, \mathbf{R}_{\perp}^{(+)}:=\mathbf{R}_{\perp}^{(\mathrm{n}+1)}, N:=N_{\mathrm{n}}$, etc. We conjugate $\mathbf{L}$ in (8.14) by a transformation of the form

$$
\boldsymbol{\Phi}:=e^{\mathbf{X}}, \mathbf{X}:=\left(\frac{X^{(d)}}{X^{(o)}} \frac{X^{(o)}}{X^{(d)}}\right), X^{(d)}: H_{\mathbb{S}_{0}}^{\perp} \rightarrow H_{\mathbb{S}_{0}}^{\perp}, X^{(o)}: H_{-\mathbb{S}_{0}}^{\perp} \rightarrow H_{\mathbb{S}_{0}}^{\perp},
$$

where $\mathbf{X}$ is a bounded linear operator, chosen below in (8.37), (8.38). By the Lie expansions (3.16)-(3.17) we have

$$
\begin{aligned}
\mathbf{L}_{+} & :=\boldsymbol{\Phi}^{-1} \mathbf{L} \boldsymbol{\Phi} \\
= & \omega \cdot \partial_{\varphi} \mathbb{1}_{\perp}+\mathrm{i} \mathbf{D}+\left(\left(\omega \cdot \partial_{\varphi} \mathbf{X}\right)-\mathrm{i}[\mathbf{X}, \mathbf{D}]+\Pi_{N} \mathbf{R}_{\perp}\right)+\Pi_{N}^{\perp} \mathbf{R}_{\perp} \\
& -\int_{0}^{1} e^{-\tau \mathbf{X}}\left[\mathbf{X}, \mathbf{R}_{\perp}\right] e^{\tau \mathbf{X}} \mathrm{d} \tau-\int_{0}^{1}(1-\tau) e^{-\tau \mathbf{X}}\left[\mathbf{X},\left(\omega \cdot \partial_{\varphi} \mathbf{X}\right)-\mathrm{i}[\mathbf{X}, \mathbf{D}]\right] e^{\tau \mathbf{X}} \mathrm{d} \tau
\end{aligned}
$$

where $\Pi_{N}$ is defined in (3.39) and $\Pi_{N}^{\perp}:=\mathrm{Id}-\Pi_{N}$. We want to solve the homological equation

$$
\omega \cdot \partial_{\varphi} \mathbf{X}-\mathrm{i}[\mathbf{X}, \mathbf{D}]+\Pi_{N} \mathbf{R}_{\perp}=\left[\mathbf{R}_{\perp}\right]
$$

where

$$
\left[\mathbf{R}_{\perp}\right]:=\left(\begin{array}{cc}
{\left[R_{\perp}^{(d)}\right]} & 0 \\
0 & {\left[R_{\perp}^{(d)}\right]}
\end{array}\right), \quad\left[R_{\perp}^{(d)}\right]:=\operatorname{diag}_{j \in \mathbb{S}_{0}^{c}}\left(R_{\perp}^{(d)}\right)_{j}^{j}(0) .
$$

By (8.14), (8.17) and (8.32), the homological equation (8.34) is equivalent to the two scalar homological equations

$$
\begin{aligned}
& \omega \cdot \partial_{\varphi} X^{(d)}-\mathrm{i}\left(X^{(d)} \mathcal{D}-\mathcal{D} X^{(d)}\right)+\Pi_{N} R_{\perp}^{(d)}=\left[R_{\perp}^{(d)}\right] \\
& \omega \cdot \partial_{\varphi} X^{(o)}+\mathrm{i}\left(X^{(o)} \overline{\mathcal{D}}+\mathcal{D} X^{(o)}\right)+\Pi_{N} R_{\perp}^{(o)}=0 .
\end{aligned}
$$

Recalling (8.14) and since $\overline{\mathcal{D}}=\operatorname{diag}_{j \in-\mathbb{S}_{0}^{c}}\left(\mu_{-j}\right)$, acting in $H_{-\mathbb{S}_{0}}^{\perp}$ (see (8.4)) the solutions of (8.36) are, for all $(\omega, \kappa) \in \Lambda_{\mathrm{n}+1}^{v}$ (see (8.20) with $\left.\mathrm{n} \rightsquigarrow \mathrm{n}+1\right)$

$$
\begin{aligned}
& \left(X^{(d)}\right)_{j}^{j^{\prime}}(\ell):= \begin{cases}-\frac{\left(R_{\perp}^{(d)}\right)_{j}^{j^{\prime}}(\ell)}{\mathrm{i}\left(\omega \cdot \ell+\mu_{j}-\mu_{j^{\prime}}\right)} & \text { if }\left\{\begin{array}{l}
\left(\ell, j, j^{\prime}\right) \neq(0, j, j), j, j^{\prime} \in \mathbb{S}_{0}^{c},\langle\ell\rangle \leqq N \\
\ell \cdot \vec{j}+j-j^{\prime}=0 \\
0
\end{array}\right. \\
\text { otherwise, }\end{cases} \\
& \left(X^{(o)}\right)_{j}^{j^{\prime}}(\ell):= \begin{cases}-\frac{\left(R_{\perp}^{(o)}\right)_{j}^{j^{\prime}}(\ell)}{\mathrm{i}\left(\omega \cdot \ell+\mu_{j}+\mu_{-j^{\prime}}\right)} & \text { if }\left\{\begin{array}{l}
\forall \ell \in \mathbb{Z}^{v} j,-j^{\prime} \in \mathbb{S}_{0}^{c},\langle\ell\rangle \leqq N \\
\ell \cdot \vec{j}+j-j^{\prime}=0 \\
0
\end{array}\right. \text { otherwise. }\end{cases}
\end{aligned}
$$

Note that, since $-j^{\prime} \in \mathbb{S}_{0}^{c}$, we can apply the bounds (8.20) for $(\omega, \kappa) \in \Lambda_{\mathrm{n}+1}^{v}$. 
Lemma 8.5. (Homological equations) The real operator $\mathbf{X}$ defined in (8.32), (8.37), (8.38), (which for all $(\omega, \kappa) \in \Lambda_{\mathrm{n}+1}^{v}$ solves the homological equation (8.20))) admits an extension to the whole parameter space $\mathbb{R}^{v} \times\left[\kappa_{1}, \kappa_{2}\right]$. Such extended operator is $\mathcal{D}^{k_{0}}$-modulo-tame with a modulo-tame constant satisfying, for all $s_{0} \leqq$ $s \leqq S$,

$$
\mathfrak{M}_{\mathbf{X}}^{\sharp}(s) \lesssim k_{0} N^{\tau_{1}} v^{-1} \mathfrak{M}^{\sharp}(s), \quad \mathfrak{M}_{\left\langle\partial_{\varphi}\right\rangle}^{\sharp} \mathbf{X}^{(s) \lesssim k_{0}} N^{\tau_{1}} v^{-1} \mathfrak{M}^{\sharp}(s, \mathrm{~b}),
$$

where $\tau_{1}:=\tau\left(k_{0}+1\right)+k_{0}$. If $v / 2 \leqq v_{1}, v_{2} \leqq 2 v$, then, for all $(\omega, \kappa) \in$ $\Lambda_{\mathrm{n}+1}^{v_{1}}\left(i_{1}\right) \cap \Lambda_{\mathrm{n}+1}^{v_{2}}\left(i_{2}\right)$,

$\left\|\left|\Delta_{12} \mathbf{X}\right|\right\|_{\mathcal{L}\left(H^{s_{0}}\right)} \lesssim N^{2 \tau} v^{-1}\left(\left\|\left|\mathbf{R}_{\perp}\left(i_{2}\right)\right|\right\|_{\mathcal{L}\left(H^{s_{0}}\right)}\left\|i_{1}-i_{2}\right\|_{s_{0}+\mu(\mathrm{b})}+\left\|\left|\Delta_{12} \mathbf{R}_{\perp}\right|\right\|_{\mathcal{L}\left(H^{s_{0}}\right)}\right)$

$\left\|\left|\left\langle\partial_{\varphi}\right\rangle^{\mathrm{b}} \Delta_{12} \mathbf{X}\right|\right\|_{\mathcal{L}\left(H^{s}\right)} \lesssim$

$N^{2 \tau} v^{-1}\left(\left\|\left|\left\langle\partial_{\varphi}\right\rangle^{\mathrm{b}} \mathbf{R}_{\perp}\left(i_{2}\right)\right|\right\|_{\mathcal{L}\left(H^{s_{0}}\right)}\left\|i_{1}-i_{2}\right\|_{s_{0}+\mu(\mathrm{b})}+\left\|\left|\left\langle\partial_{\varphi}\right\rangle^{\mathrm{b}} \Delta_{12} \mathbf{R}_{\perp}\right|\right\|_{\mathcal{L}\left(H^{s_{0}}\right)}\right)$.

The operator $\mathbf{X}$ is reversibility and momentum preserving.

Proof. We prove that (8.39) holds for $X^{(d)}$. The proof for $X^{(o)}$ holds analogously. First, we extend the solution in (8.37) to all $\lambda$ in $\mathbb{R}^{v} \times\left[\kappa_{1}, \kappa_{2}\right]$ by setting (without any further relabeling $\left(X^{(d)}\right)_{j}^{j^{\prime}}(\ell)=\mathrm{i} g_{\ell, j, j^{\prime}}(\lambda)\left(R_{\perp}^{(d)}\right)_{j}^{j^{\prime}}(\ell)$, where

$g_{\ell, j, j^{\prime}}(\lambda):=\frac{\chi\left(f(\lambda) \rho^{-1}\right)}{f(\lambda)}, \quad f(\lambda):=\omega \cdot \ell+\mu_{j}-\mu_{j^{\prime}}, \quad \rho:=v\langle\ell\rangle^{-\tau}\left\langle|j|^{\frac{3}{2}}-\left|j^{\prime}\right|^{\frac{3}{2}}\right\rangle$,

and $\chi$ is the cut-off function (3.10). By (8.15), (8.16), (7.188), (8.20), Lemma 4.4, (5.39), together with (3.10), we deduce that, for any $k_{1} \in \mathbb{N}_{0}^{v},\left|k_{1}\right| \leqq k_{0}$,

$$
\sup _{\left|k_{1}\right| \leqq k_{0}}\left|\partial_{\lambda}^{k_{1}} g_{\ell, j, j^{\prime}}\right| \lesssim k_{0}\langle\ell\rangle^{\tau_{1}} v^{-1-\left|k_{1}\right|}, \quad \tau_{1}=\tau\left(k_{0}+1\right)+k_{0},
$$

and we deduce, for all $0 \leqq|k| \leqq k_{0}$,

$$
\begin{aligned}
\left|\partial_{\lambda}^{k}\left(X^{(d)}\right)_{j}^{j^{\prime}}(\ell)\right| & \lesssim_{k_{0}} \sum_{k_{1}+k_{2}=k}\left|\partial_{\lambda}^{k_{1}} g_{\ell, j, j^{\prime}}(\lambda)\right|\left|\partial_{\lambda}^{k_{2}}\left(R_{\perp}^{(d)}\right)_{j}^{j^{\prime}}(\ell)\right| \\
& \lesssim_{k_{0}}\langle\ell\rangle^{\tau_{1}} v^{-1-|k|} \sum_{\left|k_{2}\right| \leqq|k|} v^{\left|k_{2}\right|}\left|\partial_{\lambda}^{k_{2}}\left(R_{\perp}^{(d)}\right)_{j}^{j^{\prime}}(\ell)\right| .
\end{aligned}
$$

By (8.37) we have that $\left(X^{(d)}\right)_{j}^{j^{\prime}}(\ell)=0$ for all $\langle\ell\rangle>N$. Therefore, for all $|k| \leqq k_{0}$, we have

$$
\begin{aligned}
& \left\|\left|\left\langle\partial_{\varphi}\right\rangle^{\mathrm{b}} \partial_{\lambda}^{k} X^{(d)}\right| h\right\|_{s}^{2} \leqq \sum_{\ell, j}\langle\ell, j\rangle^{2 s}\left(\sum_{\left\langle\ell-\ell^{\prime}\right\rangle \leqq N, j^{\prime}}\left|\left\langle\ell-\ell^{\prime}\right\rangle^{\mathrm{b}} \partial_{\lambda}^{k}\left(X^{(d)}\right)_{j}^{j^{\prime}}\left(\ell-\ell^{\prime}\right) \| h_{\ell^{\prime}, j^{\prime}}\right|\right)^{2} \\
& \stackrel{(8.42)}{\lesssim} N_{0}^{2 \tau_{1}} v^{-2(1+|k|)} \sum_{\left|k_{2}\right| \leqq|k|} v^{2\left|k_{2}\right|} \sum_{\ell, j}\langle\ell, j\rangle^{2 s}\left(\sum_{\ell^{\prime}, j^{\prime}}\left|\left\langle\ell-\ell^{\prime}\right\rangle^{\mathrm{b}} \partial_{\lambda}^{k_{2}}\left(R_{\perp}^{(d)}\right)_{j}^{j^{\prime}}\left(\ell-\ell^{\prime}\right)\right|\left|h_{\ell^{\prime}, j^{\prime}}\right|\right)^{2}
\end{aligned}
$$




$$
\begin{aligned}
& \lesssim_{k_{0}} N^{2 \tau_{1}} v^{-2(1+|k|)} \sum_{\left|k_{2}\right| \leqq|k|} v^{2\left|k_{2}\right|}\left\|\left|\left\langle\partial_{\varphi}\right\rangle^{\mathrm{b}} \partial_{\lambda}^{k_{2}} R_{\perp}^{(d)}\|h \mid\|_{s}^{2}\right.\right. \\
& \stackrel{(3.38),(8.18)}{\lesssim k_{0}} N^{2 \tau_{1}} v^{-2(1+|k|)}\left(\mathfrak{M}^{\sharp}(s, \mathrm{~b})^{2}\|h\|_{s_{0}}^{2}+\mathfrak{M}^{\sharp}\left(s_{0}, \mathrm{~b}\right)^{2}\|h\|_{s}^{2}\right),
\end{aligned}
$$

and, by Definition 3.14, we conclude that $\mathfrak{M}_{\left\langle\partial_{\varphi}\right\rangle^{\mathrm{b}} X^{(d)}}^{\sharp}(s) \lesssim_{k_{0}} N^{\tau_{1}} v^{-1} \mathfrak{M}^{\sharp}(s, \mathrm{~b})$. The analogous estimates for $\left\langle\partial_{\varphi}\right\rangle^{\mathrm{b}} X^{(o)}, X^{(d)}, X^{(o)}$ and (8.40), (8.41) follow similarly. By induction, the operator $\mathbf{R}_{\perp}$ is reversible and momentum preserving. Therefore, by (8.32), (8.37), (8.38) and Lemmata 3.18, 3.28, it follows that $\mathbf{X}$ is reversibility and momentum preserving.

By (8.33), (8.34), for all $\lambda \in \Lambda_{\mathrm{n}+1}^{v}$, we have

$$
\mathbf{L}_{+}=\boldsymbol{\Phi}^{-1} \mathbf{L} \boldsymbol{\Phi}=\omega \cdot \partial_{\varphi} \mathbb{1}_{\perp}+\mathrm{i} \mathbf{D}_{+}+\mathbf{R}_{\perp}^{(+)},
$$

where

$$
\begin{aligned}
\mathbf{D}_{+}:= & \mathbf{D}-\mathrm{i}\left[\mathbf{R}_{\perp}\right], \\
\mathbf{R}_{\perp}^{(+)}:= & \Pi_{N}^{\perp} \mathbf{R}_{\perp}-\int_{0}^{1} e^{\left.-\tau \mathbf{X}_{[\mathbf{X}}, \mathbf{R}_{\perp}\right] e^{\tau \mathbf{X}} \mathrm{d} \tau} \\
& +\int_{0}^{1}(1-\tau) e^{-\tau \mathbf{X}}\left[\mathbf{X}, \Pi_{N} \mathbf{R}_{\perp}-\left[\mathbf{R}_{\perp}\right]\right] e^{\tau \mathbf{X}} \mathrm{d} \tau .
\end{aligned}
$$

The right hand side of (8.43)-(8.44) define an extension of $\mathbf{L}_{+}$to the whole parameter space $\mathbb{R}^{v} \times\left[\kappa_{1}, \kappa_{2}\right]$, since $\mathbf{R}_{\perp}$ and $\mathbf{X}$ are defined on $\mathbb{R}^{v} \times\left[\kappa_{1}, \kappa_{2}\right]$.

The new operator $\mathbf{L}_{+}$in (8.43) has the same form of $\mathbf{L}$ in (8.14) with the nondiagonal remainder $\mathbf{R}_{\perp}^{(+)}$which is the sum of a term $\Pi_{N}^{\perp} \mathbf{R}_{\perp}$ supported on high frequencies and a quadratic function of $\mathbf{X}$ and $\mathbf{R}_{\perp}$. The new normal form $\mathbf{D}_{+}$is diagonal:

Lemma 8.6. (New diagonal part) For all $(\omega, \kappa) \in \mathbb{R}^{v} \times\left[\kappa_{1}, \kappa_{2}\right]$, the new normal form is

$$
\mathrm{i} \mathbf{D}_{+}=\mathrm{i} \mathbf{D}+\left[\mathbf{R}_{\perp}\right]=\mathrm{i}\left(\begin{array}{cc}
\mathcal{D}_{+} & 0 \\
0 & -\overline{\mathcal{D}_{+}}
\end{array}\right), \mathcal{D}_{+}:=\operatorname{diag}_{j \in \mathbb{S}_{0}^{c}} \mu_{j}^{(+)}, \mu_{j}^{(+)}:=\mu_{j}+r_{j} \in \mathbb{R},
$$

where each $\Upsilon_{j}$ satisfies, on $\mathbb{R}^{v} \times\left[\kappa_{1}, \kappa_{2}\right]$,

$$
\left|\Upsilon_{j}\right|^{k_{0}, v}=\left|\mu_{j}^{(+)}-\mu_{j}\right|^{k_{0}, v} \lesssim \mathfrak{M}^{\sharp}\left(s_{0}\right) .
$$

Moreover, given tori $i_{1}(\omega, \kappa), i_{2}(\omega, \kappa)$, we have $\left|r_{j}\left(i_{1}\right)-r_{j}\left(i_{2}\right)\right| \lesssim\left\|\left|\Delta_{12} \mathbf{R}_{\perp}\right|\right\|_{\mathcal{L}\left(H^{s_{0}}\right)}$.

Proof. Recalling (8.35), we have that $r_{j}:=-\mathrm{i}\left(R_{\perp}^{(d)}\right)_{j}^{j}(0)$, for all $j \in \mathbb{S}_{0}^{c}$. By the reversibility of $R_{\perp}^{(d)}$ and (3.44) we deduce that $r_{j} \in \mathbb{R}$. Recalling the definition of $\mathfrak{M}^{\sharp}\left(s_{0}\right)$ in (8.18) (with $s=s_{0}$ ) and Definition 3.14, we have, for all $0 \leqq$ $|k| \leqq k_{0},\left\|\left|\partial_{\lambda}^{k} R_{\perp}^{(d)}\right| h\right\|_{s_{0}} \leqq 2 v^{-|k|} \mathfrak{M}^{\sharp}\left(s_{0}\right)\|h\|_{s_{0}}$, and therefore $\left|\partial_{\lambda}^{k}\left(R_{\perp}^{(d)}\right)_{j}^{j}(0)\right| \lesssim$ $v^{-|k|} \mathfrak{M}^{\sharp}\left(s_{0}\right)$. Hence (8.45) follows. The last bound for $\left|r_{j}\left(i_{1}\right)-r_{j}\left(i_{2}\right)\right|$ follows analogously. 
The iterative step. Let $\mathrm{n} \in \mathbb{N}_{0}$ and assume that the statements $(\mathbf{S 1})_{\mathrm{n}}-(\mathbf{S 3})_{\mathrm{n}}$ are true. We now prove $(\mathbf{S 1})_{\mathrm{n}+1}-(\mathbf{S 3})_{\mathrm{n}+1}$. For sake of simplicity in the notation (as in other parts of the paper) we omit to write the dependence on $k_{0}$, which is considered as a fixed constant.

Proof of $(\mathbf{S 1})_{\mathrm{n}+1}$. The real operator $\mathbf{X}_{\mathrm{n}}$ defined in Lemma 8.5 is defined for all $(\omega, \kappa) \in \mathbb{R}^{v} \times\left[\kappa_{1}, \kappa_{2}\right]$ and, by (8.39), (8.19), satisfies the estimates (8.22) at the step $\mathrm{n}+1$. The flow maps $\boldsymbol{\Phi}_{\mathrm{n}}^{ \pm 1}=e^{ \pm \mathbf{X}_{\mathrm{n}}}$ are well defined by Lemma 3.16. By (8.43), for all $\lambda \in \Lambda_{\mathrm{n}+1}^{v}$, the conjugation formula (8.21) holds at the step $\mathrm{n}+1$. The operator $\mathbf{X}_{\mathrm{n}}$ is reversibility and momentum preserving, and so are the operators $\boldsymbol{\Phi}_{\mathrm{n}}^{ \pm 1}=e^{ \pm \mathbf{X}_{\mathrm{n}}}$. By Lemma 8.6, the operator $\mathbf{D}_{\mathrm{n}+1}$ is diagonal with eigenvalues $\mu_{j}^{(\mathrm{n}+1)}: \mathbb{R}^{v} \times\left[\kappa_{1}, \kappa_{2}\right] \rightarrow \mathbb{R}, \mu_{j}^{(\mathrm{n}+1)}=\mu_{j}^{(0)}+\mathfrak{r}_{j}^{(\mathrm{n}+1)}$ with $\mathfrak{r}_{j}^{(\mathrm{n}+1)}:=\mathfrak{r}_{j}^{(\mathrm{n})}+r_{j}^{(\mathrm{n})}$ satisfying, using also (8.19), (8.16) at the step $n+1$. The next lemma provides the estimates of the remainder $\mathbf{R}_{\perp}^{(\mathrm{n}+1)}=\mathbf{R}_{\perp}^{(+)}$defined in (8.44).

Lemma 8.7. The operators $\mathbf{R}_{\perp}^{(\mathrm{n}+1)}$ and $\left\langle\partial_{\varphi}\right\rangle^{\mathrm{b}} \mathbf{R}_{\perp}^{(\mathrm{n}+1)}$ are $\mathcal{D}^{k_{0}}$-modulo-tame with modulo-tame constants satisfying

$$
\begin{aligned}
& \mathfrak{M}_{\mathrm{n}+1}^{\sharp}(s) \lesssim N_{\mathrm{n}}^{-\mathrm{b}} \mathfrak{M}_{\mathrm{n}}^{\sharp}(s, \mathrm{~b})+N_{\mathrm{n}}^{\tau_{1}} v^{-1} \mathfrak{M}_{\mathrm{n}}^{\sharp}(s) \mathfrak{M}_{\mathrm{n}}^{\sharp}\left(s_{0}\right), \\
& \mathfrak{M}_{\mathrm{n}+1}^{\sharp}(s, \mathrm{~b}) \lesssim \mathfrak{M}_{\mathrm{n}}^{\sharp}(s, \mathrm{~b})+N_{\mathrm{n}}^{\tau_{1}} v^{-1}\left(\mathfrak{M}_{\mathrm{n}}^{\sharp}(s, \mathrm{~b}) \mathfrak{M}_{\mathrm{n}}^{\sharp}\left(s_{0}\right)+\mathfrak{M}_{\mathrm{n}}^{\sharp}\left(s_{0}, \mathrm{~b}\right) \mathfrak{M}_{\mathrm{n}}^{\sharp}(s)\right) .
\end{aligned}
$$

Proof. The estimates (8.46), (8.47) follow by (8.44), Lemmata 3.15, 3.16, the bounds (3.40) and (8.39), (8.19), (8.6), (8.12), (8.13).

Lemma 8.8. Estimates (8.19) holds at the step $\mathrm{n}+1$.

Proof. It follows by $(8.46),(8.47),(8.19)$ at the step $n,(8.6)$, the smallness condition (8.13) with $N_{0}=N_{0}\left(s_{0}, \mathrm{~b}\right)>0$ large enough and taking $\tau_{2}>\tau_{1}+\mathrm{a}$.

Finally $\mathbf{R}_{\perp}^{(\mathrm{n}+1)}$ is real, reversible and momentum preserving as $\mathbf{R}_{\perp}^{(\mathrm{n})}$, since $\mathbf{X}_{\mathrm{n}}$ is real, reversibility and momentum preserving. This concludes the proof of $(\mathbf{S 1})_{\mathrm{n}+1}$.

Proof of $(\mathbf{S 2})_{\mathrm{n}+1}$. It follows by similar arguments and we omit it.

Proof OF $(\mathbf{S 3})_{\mathrm{n}+1}$. The proof follows as for $(\mathbf{S 4})_{v+1}$ of Theorem 7.3 in [6], using (S2) $)_{\mathrm{n}}$ and the fact that the momentum condition in (8.20) implies $\left|j-j^{\prime}\right| \lesssim N_{\mathrm{n}}$.

\section{Almost invertibility of $\mathcal{L}_{\omega}$}

By (7.183) and Theorem 8.3 (where $\mathbf{L}_{0}=\mathcal{L}_{\perp}$ ) we obtain

$$
\mathcal{L}_{\omega}=\mathbf{W}_{2, \overline{\mathrm{n}}} \mathbf{L}_{\overline{\mathrm{n}}} \mathbf{W}_{1, \overline{\mathrm{n}}}^{-1}, \quad \mathbf{W}_{1, \overline{\mathrm{n}}}:=\mathcal{W}_{1}^{\perp} \mathbf{U}_{\overline{\mathrm{n}}}, \quad \mathbf{W}_{2, \overline{\mathrm{n}}}:=\mathcal{W}_{2}^{\perp} \mathbf{U}_{\overline{\mathrm{n}}},
$$

where the operator $\mathbf{L}_{\overline{\mathrm{n}}}$ is defined in (8.14) with $\mathrm{n}=\overline{\mathrm{n}}$. By (7.181) and (8.30), we have, for some $\sigma:=\sigma\left(\tau, v, k_{0}\right)>0$, for any $s_{0} \leqq s \leqq S$,

$$
\left\|\mathbf{W}_{1, \overline{\mathrm{n}}}^{ \pm 1} h\right\|_{S}^{k_{0}, v},\left\|\mathbf{W}_{2, \overline{\mathrm{n}}}^{ \pm 1} h\right\|_{S}^{k_{0}, v} \lesssim_{S}\|h\|_{s+\sigma}^{k_{0}, v}+\left\|\mathfrak{I}_{0}\right\|_{s+\mu(\mathrm{b})+\sigma}^{k_{0}, v}\|h\|_{s_{0}+\sigma}^{k_{0}, v} .
$$


In order to verify the almost invertibility assumption (AI) of $\mathcal{L}_{\omega}$ in Section 6, we decompose the operator $\mathbf{L}_{\overline{\mathrm{n}}}$ in (8.14) (with $\overline{\mathrm{n}}$ instead of $\mathrm{n}$ ) as

$$
\mathbf{L}_{\overline{\mathrm{n}}}=\mathbf{D}_{\overline{\mathrm{n}}}^{<}+\mathbf{Q}_{\perp}^{(\overline{\mathrm{n}})}+\mathbf{R}_{\perp}^{(\overline{\mathrm{n}})}
$$

where

$$
\begin{aligned}
& \mathbf{D}_{\overline{\mathrm{n}}}^{<}:=\Pi_{K_{\overline{\mathrm{n}}}}\left(\omega \cdot \partial_{\varphi} \mathbb{1}_{\perp}+\mathrm{i} \mathbf{D}_{\overline{\mathrm{n}}}\right) \Pi_{K_{\overline{\mathrm{n}}}}+\Pi_{K_{\overline{\mathrm{n}}}}^{\perp}, \\
& \mathbf{Q}_{\perp}^{(\overline{\mathrm{n}})}:=\Pi_{K_{\overline{\mathrm{n}}}}^{\perp}\left(\omega \cdot \partial_{\varphi} \mathbb{1}_{\perp}+\mathrm{i} \mathbf{D}_{\overline{\mathrm{n}}}\right) \Pi_{K_{\overline{\mathrm{n}}}}^{\perp}-\Pi_{K_{\overline{\mathrm{n}}}}^{\perp},
\end{aligned}
$$

and the smoothing operator $\Pi_{K}$ on the traveling waves is defined in (3.6), and $\Pi_{K}^{\perp}:=\mathrm{Id}-\Pi_{K}$. The constants $K_{\overline{\mathrm{n}}}$ in (8.51) are $K_{\overline{\mathrm{n}}}:=K_{0}^{\chi^{\overline{\mathrm{n}}}}, \chi=3 / 2(\mathrm{cfr}$. (6.24)), and $K_{0}$ will be fixed in (9.5).

Lemma 8.9. (First order Melnikov non-resonance conditions) For all $\lambda=(\omega, \kappa)$ in

$$
\begin{aligned}
\Lambda_{\overline{\mathrm{n}}+1}^{v, I}:=\left\{\begin{array}{l}
\lambda \in \mathbb{R}^{v} \times\left[\kappa_{1}, \kappa_{2}\right]: \\
\end{array}\right. & \left.\left|\omega \cdot \ell+\mu_{j}^{(\overline{\mathrm{n}})}\right| \geqq 2 v \frac{|j|^{\frac{3}{2}}}{\langle\ell\rangle^{\tau}}, \quad \forall|\ell| \leqq K_{\overline{\mathrm{n}}}, j \in \mathbb{S}_{0}^{c}, j+\vec{j} \cdot \ell=0\right\},
\end{aligned}
$$

on the subspace of the traveling waves $\tau_{\varsigma} g(\varphi)=g(\varphi-\vec{J} \varsigma), \varsigma \in \mathbb{R}$, such that $g(\varphi, \cdot) \in \mathbf{H}_{\mathbb{S}_{0}}^{\perp}$, the operator $\mathbf{D}_{\overline{\mathrm{n}}}^{<}$in $(8.51)$ is invertible and there exists an extension of the inverse operator (that we denote in the same way) to the whole $\mathbb{R}^{v} \times\left[\kappa_{1}, \kappa_{2}\right]$ satisfying the estimate

$$
\left\|\left(\mathbf{D}_{\overline{\mathrm{n}}}^{<}\right)^{-1} g\right\|_{s}^{k_{0}, v} \lesssim k_{0} v^{-1}\|g\|_{s+\tau_{1}}^{k_{0}, v}, \quad \tau_{1}=k_{0}+\tau\left(k_{0}+1\right) .
$$

Moreover $\left(\mathbf{D}_{\overline{\mathrm{n}}}^{<}\right)^{-1} \mathrm{~g}$ is a traveling wave.

Proof. The estimate (8.53) follows arguing as in Lemma 8.5.

Standard smoothing properties imply that the operator $\mathbf{Q}_{\perp}^{(\overline{\mathrm{n}})}$ in (8.51) satisfies, for any traveling wave $h \in \mathbf{H}_{\mathbb{S}_{0}}^{\perp}$, for all $b>0$,

$$
\left\|\mathbf{Q}_{\perp}^{(\overline{\mathrm{n}})} h\right\|_{s_{0}}^{k_{0}, v} \lesssim K_{\overline{\mathrm{n}}}^{-b}\|h\|_{s_{0}+b+\frac{3}{2}}^{k_{0}, v}, \quad\left\|\mathbf{Q}_{\perp}^{(\overline{\mathrm{n}})} h\right\|_{s}^{k_{0}, v} \lesssim\|h\|_{s+\frac{3}{2}}^{k_{0}, v} .
$$

By the decompositions (8.48), (8.50), Theorem 8.3 (note that (6.1) and Lemma 6.2 imply (7.14)), Proposition 7.18, the fact that $\mathbf{W}_{1, \bar{n}}, \mathbf{W}_{2, \overline{\mathrm{n}}}$ map (anti)-reversible, respectively traveling, waves, into (anti)-reversible, respectively traveling, waves (Lemma 7.15) and estimates (8.49), (8.53), (8.54), (3.8) we deduce the following theorem.

Theorem 8.10. (Almost invertibility of $\mathcal{L}_{\omega}$ ) Assume (6.1). Let a, b as in (8.6) and $M$ as in (8.5). Let $S>s_{0}$ and assume the smallness condition (8.29). Then the almost invertibility assumption (AI) in Section 6 holds with $\Lambda_{0}$ replaced by

$$
\boldsymbol{\Lambda}_{\overline{\mathrm{n}}+1}^{v}:=\boldsymbol{\Lambda}_{\overline{\mathrm{n}}+1}^{v}(i):=\Lambda_{\overline{\mathrm{n}}+1}^{v} \cap \Lambda_{\overline{\mathrm{n}}+1}^{v, I}
$$

(see (8.20), (8.52)) and, with $\mu(\mathrm{b})$ defined in (8.7),

$$
\mathcal{L}_{\omega}^{<}:=\mathbf{W}_{2, \overline{\mathrm{n}}} \mathbf{D}_{\overline{\mathrm{n}}}^{<} \mathbf{W}_{1, \overline{\mathrm{n}}}^{-1}, \quad \mathcal{R}_{\omega}:=\mathbf{W}_{2, \overline{\mathrm{n}}} \mathbf{R}_{\perp}^{(\overline{\mathrm{n}})} \mathbf{W}_{1, \overline{\mathrm{n}}}^{-1}, \quad \mathcal{R}_{\omega}^{\perp}:=\mathbf{W}_{2, \overline{\mathrm{n}}} \mathbf{Q}_{\perp}^{(\overline{\mathrm{n}})} \mathbf{W}_{1, \overline{\mathrm{n}}}^{-1} .
$$




\section{Proof of Theorem $\mathbf{5 . 2}$}

Theorem 5.2 is a consequence of Theorem 9.2 below. We consider the finite dimensional subspaces of traveling wave variations

$$
E_{\mathrm{n}}:=\left\{\Im(\varphi)=(\Theta, I, w)(\varphi) \text { such that (3.53) holds : } \Theta=\Pi_{\mathrm{n}} \Theta, I=\Pi_{\mathrm{n}} I, w=\Pi_{\mathrm{n}} w\right\}
$$

where $\Pi_{\mathrm{n}} w:=\Pi_{K_{\mathrm{n}}} w$ are defined as in (3.6) with $K_{n}$ in (6.24), and we denote with the same symbol $\Pi_{\mathrm{n}} g(\varphi):=\sum_{|\ell| \leq K_{\mathrm{n}}} g_{\ell} e^{\mathrm{i} \ell \cdot \varphi}$. Note that the projector $\Pi_{\mathrm{n}}$ maps (anti)-reversible traveling variations into (anti)-reversible traveling variations.

In view of the Nash-Moser Theorem 9.2 we introduce the constants

$$
\begin{aligned}
& \mathrm{a}_{1}:=\max \left\{6 \sigma_{1}+13, \chi\left(p(\tau+1)+\mu(\mathrm{b})+2 \sigma_{1}\right)+1\right\}, \quad \mathrm{a}_{2}:=\chi^{-1} \mathrm{a}_{1}-\mu(\mathrm{b})-2 \sigma_{1}, \\
& \mu_{1}:=3\left(\mu(\mathrm{b})+2 \sigma_{1}\right)+1, \quad \mathrm{~b}_{1}:=\mathrm{a}_{1}+2 \mu(\mathrm{b})+4 \sigma_{1}+3+\chi^{-1} \mu_{1}, \quad \chi=3 / 2 \\
& \sigma_{1}:=\max \left\{\bar{\sigma}, 2 s_{0}+2 k_{0}+5\right\}, \quad S=s_{0}+\mathrm{b}_{1},
\end{aligned}
$$

where $\bar{\sigma}=\bar{\sigma}\left(\tau, v, k_{0}\right)>0$ is defined by Theorem $6.6,2 s_{0}+2 k_{0}+5$ is the largest loss of regularity in the estimates of the Hamiltonian vector field $X_{P}$ in Lemma $6.1, \mu(\mathrm{b})$ is defined in (8.7), and $\mathrm{b}=[\mathrm{a}]+2$ is defined in (8.6). The exponent $p$ in (6.24) is required to satisfy

$$
p a>\frac{1}{2} a_{1}+\frac{3}{2} \sigma_{1} .
$$

By (8.6), and the definition of $\mathrm{a}_{1}$ in (9.1), there exists $p=p\left(\tau, v, k_{0}\right)$ such that (9.4) holds, for example we fix

$$
p:=\frac{3\left(\mu(\mathrm{b})+4 \sigma_{1}+1\right)}{a} .
$$

Remark 9.1. The constant $a_{1}$ is the exponent in (9.9). The constant $a_{2}$ is the exponent in the second bound in (9.7). The constant $\mu_{1}$ is the exponent in $(\mathcal{P} 3)_{\mathrm{n}}$. The conditions on the constants $\mu_{1}, \mathrm{~b}_{1}, \mathrm{a}_{1}$ to allow the convergence of the NashMoser scheme in Theorem 9.2 are

$$
\mathrm{a}_{1}>6 \sigma_{1}+12, \quad \mathrm{~b}_{1}>\mathrm{a}_{1}+2 \mu(\mathrm{b})+4 \sigma_{1}+\chi^{-1} \mu_{1}, \quad p \mathrm{a}>\frac{1}{2} \mathrm{a}_{1}+\frac{3}{2} \sigma_{1},
$$

as well as $\mu_{1}>3\left(\mu(\mathrm{b})+2 \sigma_{1}\right)$. In addition, we require $\mathrm{a}_{1} \geqq \chi(p(\tau+1)+\mu(\mathrm{b})+$ $\left.2 \sigma_{1}\right)+1$ so that $a_{2} \geqq p(\tau+1)+\chi^{-1}$, which is used in the proof of Lemma 9.3.

Given a function $W=(\mathfrak{I}, \beta)$ where $\mathfrak{I}$ is the periodic component of a torus as in (5.8) and $\beta \in \mathbb{R}^{v}$, we denote $\|W\|_{s}^{k_{0}, v}:=\|\Im\|_{s}^{k_{0}, v}+|\beta|^{k_{0}, v}$.

Theorem 9.2. (Nash-Moser) There exist $\delta_{0}, C_{*}>0$ such that, if

$$
\begin{aligned}
& K_{0}^{\tau_{3}} \varepsilon v^{-2}<\delta_{0}, \tau_{3}:=\max \left\{p \tau_{2}, 2 \sigma_{1}+\mathrm{a}_{1}+4\right\}, \\
& K_{0}:=v^{-1}, v:=\varepsilon^{\mathrm{a}}, 0<\mathrm{a}<\left(2+\tau_{3}\right)^{-1},
\end{aligned}
$$

where $\tau_{2}=\tau_{2}(\tau, v)$ is given by Theorem 8.2 , then, for all $\mathrm{n} \geqq 0$ : 
$(\mathcal{P} 1)_{\mathrm{n}}$ There exists a $k_{0}$-times differentiable function $\tilde{W}_{\mathrm{n}}: \mathbb{R}^{v} \times\left[\kappa_{1}, \kappa_{2}\right] \rightarrow$ $E_{\mathrm{n}-1} \times \mathbb{R}^{v}, \lambda=(\omega, \kappa) \mapsto \widetilde{W}_{\mathrm{n}}(\lambda):=\left(\widetilde{\mathfrak{I}}_{\mathrm{n}}, \widetilde{\alpha}_{\mathrm{n}}-\omega\right)$, for $\mathrm{n} \geqq 1$, and $\widetilde{W}_{0}:=0$, satisfying

$$
\left\|\widetilde{W}_{\mathrm{n}}\right\|_{s_{0}+\mu(\mathrm{b})+\sigma_{1}}^{k_{0}, v} \leqq C_{*} \varepsilon v^{-1} .
$$

Let $\widetilde{U}_{\mathrm{n}}:=U_{0}+\widetilde{W}_{\mathrm{n}}$, where $U_{0}:=(\varphi, 0,0, \omega)$. The difference $\widetilde{H}_{\mathrm{n}}:=\widetilde{U}_{\mathrm{n}}-$ $\widetilde{U}_{\mathrm{n}-1}$, for $\mathrm{n} \geqq 1$, satisfies

$$
\left\|\tilde{H}_{1}\right\|_{s_{0}+\mu(\mathrm{b})+\sigma_{1}}^{k_{0}, v} \leqq C_{*} \varepsilon v^{-1},\left\|\widetilde{H}_{\mathrm{n}}\right\|_{s_{0}+\mu(\mathrm{b})+\sigma_{1}}^{k_{0}, v} \leqq C_{*} \varepsilon v^{-1} K_{\mathrm{n}-1}^{-\mathrm{a}_{2}}, \forall \mathrm{n} \geqq 2 .
$$

The torus embedding $\widetilde{l}_{\mathrm{n}}:=(\varphi, 0,0)+\widetilde{\mathfrak{I}}_{\mathrm{n}}$ is reversible and traveling, that is (5.7) holds.

$(\mathcal{P} 2)_{\mathrm{n}}$ We define

$$
\mathcal{G}_{0}:=\Omega \times\left[\kappa_{1}, \kappa_{2}\right], \quad \mathcal{G}_{\mathrm{n}+1}:=\mathcal{G}_{\mathrm{n}} \cap \Lambda_{\mathrm{n}+1}^{v}\left(\widetilde{l}_{\mathrm{n}}\right), \quad \forall \mathrm{n} \geqq 0,
$$

where $\boldsymbol{\Lambda}_{\mathrm{n}+1}^{v}\left(\widetilde{l}_{\mathrm{n}}\right)$ is defined in (8.55). Then, for all $\lambda \in \mathcal{G}_{\mathrm{n}}$, setting $K_{-1}:=1$, we have

$$
\left\|\mathcal{F}\left(\widetilde{U}_{\mathrm{n}}\right)\right\|_{s_{0}}^{k_{0}, v} \leqq C_{*} \varepsilon K_{\mathrm{n}-1}^{-\mathrm{a}_{1}}
$$

$(\mathcal{P} 3)_{\mathrm{n}}$ (High NORMs) For all $\lambda \in \mathcal{G}_{\mathrm{n}}$, we have $\left\|\widetilde{W}_{\mathrm{n}}\right\|_{s_{0}+\mathrm{b}_{1}}^{k_{0}, v} \leqq C_{*} \varepsilon v^{-1} K_{\mathrm{n}-1}^{\mu_{1}}$.

Proof. The inductive proof follows exactly as in $[2,6]$. Note that the almost invertibility property proved in Theorem 8.10, as well as in Theorem 6.6, is formulated exactly as in $[2,6]$. The only novelty is to check that each approximate torus $\tilde{l}_{\mathrm{n}}$ is reversible and traveling. Clearly $i_{0}:=(\varphi, 0,0)$ satisfies (5.7). Supposing inductively that $\tilde{l}_{\mathrm{n}}$ is reversible and traveling, we now prove that the successive approximation $\tilde{\imath}_{\mathrm{n}+1}$ defined by the modified Nash-Moser scheme in [2,6] is a reversible and traveling wave as well. By (9.5), the smallness condition (8.29) holds for $\varepsilon$ small enough. Moreover (6.1) holds by (9.6). Therefore Theorem 8.10 holds and the almost invertibility assumption (AI) of Section 6 holds for all $\lambda \in \Lambda_{\mathrm{n}+1}^{v}$, see (8.55). Then Theorem 6.6 implies the existence of an almost approximate in-

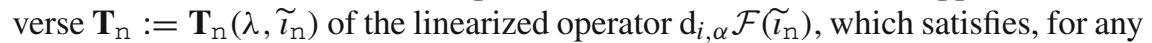
anti-reversible traveling wave variation $g$, the tame estimate (6.40). Moreover. the first three components of $\mathbf{T}_{\mathrm{n}} g$ form a reversible traveling wave variation. For all $\lambda \in \mathcal{G}_{\mathrm{n}+1}=\mathcal{G}_{\mathrm{n}} \cap \Lambda_{\mathrm{n}+1}^{v}\left(\widetilde{l}_{\mathrm{n}}\right)$ (cfr. (9.8)) we define the successive approximation

$$
U_{\mathrm{n}+1}:=\widetilde{U}_{\mathrm{n}}+H_{\mathrm{n}+1}, \quad H_{\mathrm{n}+1}:=\left(\widehat{\widetilde{I}}_{\mathrm{n}+1}, \widehat{\alpha}_{\mathrm{n}+1}\right):=-\boldsymbol{\Pi}_{\mathrm{n}} \mathbf{T}_{\mathrm{n}} \Pi_{\mathrm{n}} \mathcal{F}\left(\widetilde{U}_{\mathrm{n}}\right) \in E_{\mathrm{n}} \times \mathbb{R}^{v}
$$

where $\Pi_{\mathrm{n}}$ is defined for any $(\mathfrak{I}, \alpha)$, with $\mathfrak{I}$ a traveling wave variation, by $\Pi_{\mathrm{n}}(\mathfrak{I}, \alpha):=$ $\left(\Pi_{\mathrm{n}} \mathfrak{I}, \alpha\right)$. By Lemma 5.1 and since $\widetilde{\imath}_{\mathrm{n}}$ is a reversible traveling wave, we have that $\mathcal{F}\left(\widetilde{U}_{\mathrm{n}}\right)=\mathcal{F}\left(\widetilde{l}_{\mathrm{n}}, \widetilde{\alpha}_{n}\right)$ is an anti-reversible traveling wave variation, i.e (6.29)-(6.30) hold. Thus the first three components of $\mathbf{T}_{\mathrm{n}} \Pi_{\mathrm{n}} \mathcal{F}\left(\widetilde{U}_{\mathrm{n}}\right)$ form a reversible traveling wave variation, as well as $\Pi_{\mathrm{n}} \mathbf{T}_{\mathrm{n}} \Pi_{\mathrm{n}} \mathcal{F}\left(\widetilde{U}_{\mathrm{n}}\right)$. Finally one extends $H_{\mathrm{n}+1}$, defined for

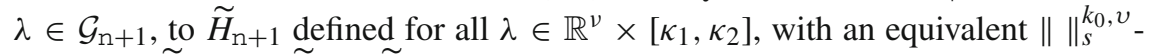
norm. Set $\widetilde{U}_{\mathrm{n}+1}:=\widetilde{U}_{\mathrm{n}}+\widetilde{H}_{\mathrm{n}+1}$.

The estimates $(9.6)-(9.9)$ and $(\mathcal{P} 3)_{n+1}$ follow exactly as in $[2,6]$. 
Proof of Theorem 5.2. Let $v=\varepsilon^{\mathrm{a}}$, with $0<\mathrm{a}<\mathrm{a}_{0}:=1 /\left(2+\tau_{3}\right)$. Then, the smallness condition in (9.5) holds for $0<\varepsilon<\varepsilon_{0}$ small enough and Theorem 9.2 holds. By (9.7), the sequence of functions $\widetilde{W}_{\mathrm{n}}=\widetilde{U}_{\mathrm{n}}-(\varphi, 0,0, \omega)=\left(\widetilde{\widetilde{I}}_{\mathrm{n}}, \widetilde{\alpha}_{\mathrm{n}}-\omega\right)$ converges to a function $W_{\infty}: \mathbb{R}^{v} \times\left[\kappa_{1}, \kappa_{2}\right] \rightarrow H_{\varphi}^{s_{0}} \times H_{\varphi}^{s_{0}} \times H^{s_{0}} \times \mathbb{R}^{v}$, and we define

$$
U_{\infty}:=\left(i_{\infty}, \alpha_{\infty}\right):=(\varphi, 0,0, \omega)+W_{\infty}
$$

The torus $i_{\infty}$ is reversible and traveling, that is (5.7) holds. By (9.6), (9.7), we also deduce

$$
\left\|U_{\infty}-U_{0}\right\|_{s_{0}+\mu(\mathrm{b})+\sigma_{1}}^{k_{0}, v} \leqq C_{*} \varepsilon v^{-1},\left\|U_{\infty}-\widetilde{U}_{\mathrm{n}}\right\|_{S_{0}+\mu(\mathrm{b})+\sigma_{1}}^{k_{0}, v} \leqq C \varepsilon v^{-1} K_{\mathrm{n}}^{-\mathrm{a} 2}, \quad \forall \mathrm{n} \geqq 1 .
$$

In particular (5.10)-(5.11) hold.

By Theorem 9.2-( $\mathcal{P} 2)_{\mathrm{n}}$, we deduce that $\mathcal{F}\left(\lambda ; U_{\infty}(\lambda)\right)=0$ for any

$$
\lambda \in \bigcap_{\mathrm{n} \in \mathbb{N}_{0}} \mathcal{G}_{\mathrm{n}}=\mathcal{G}_{0} \cap \bigcap_{\mathrm{n} \geqq 1} \Lambda_{\mathrm{n}}^{v}\left(\tilde{l}_{\mathrm{n}-1}\right) \stackrel{(8.55)}{=} \mathcal{G}_{0} \cap\left[\bigcap_{\mathrm{n} \geqq 1} \Lambda_{\mathrm{n}}^{v}\left(\tilde{l}_{\mathrm{n}-1}\right)\right] \cap\left[\bigcap_{\mathrm{n} \geqq 1} \Lambda_{\mathrm{n}}^{v, I}\left(\tilde{l}_{\mathrm{n}-1}\right)\right]
$$

where $\mathcal{G}_{0}:=\Omega \times\left[\kappa_{1}, \kappa_{2}\right]$. To conclude the proof of Theorem 5.2 it remains only to define the $\mu_{j}^{\infty}$ in (5.12) and prove that the set $\mathcal{C}_{\infty}^{v}$ in (5.14)-(5.17) is contained in $\cap_{\mathrm{n} \geqq 0} \mathcal{G}_{\mathrm{n}}$. We first define

$$
\mathcal{G}_{\infty}:=\mathcal{G}_{0} \cap\left[\bigcap_{\mathrm{n} \geqq 1} \Lambda_{\mathrm{n}}^{2 v}\left(i_{\infty}\right)\right] \cap\left[\bigcap_{\mathrm{n} \geqq 1} \Lambda_{\mathrm{n}}^{2 v, I}\left(i_{\infty}\right)\right] .
$$

Lemma 9.3. $\mathcal{G}_{\infty} \subseteq \cap_{\mathrm{n} \geqq 0} \mathcal{G}_{\mathrm{n}}$, where $\mathcal{G}_{\mathrm{n}}$ are defined in (9.8).

Proof. We shall use the inclusion property (8.27), with $S$ fixed in (9.3). By (9.10) we have

$$
\varepsilon(2 v)^{-1} C(S) N_{0}^{\tau+1}\left\|i_{\infty}-i_{0}\right\|_{s_{0}+\mu(\mathrm{b})} \leqq \varepsilon(2 v)^{-1} C(S) K_{0}^{p(\tau+1)} C_{*} \varepsilon v^{-1} \leqq v,
$$

and $\forall \mathrm{n} \geqq 2$,

$\varepsilon(2 v)^{-1} C(S) N_{\mathrm{n}-1}^{\tau+1}\left\|i_{\infty}-\tilde{l}_{\mathrm{n}-1}\right\|_{s_{0}+\mu(\mathrm{b})} \leqq \varepsilon(2 v)^{-1} C(S) K_{\mathrm{n}-1}^{p(\tau+1)} C \varepsilon v^{-1} K_{\mathrm{n}-1}^{-\mathrm{a}_{2}} \leqq v$, since $\tau_{3}>p(\tau+1)$ (by (9.5) and $\left.\tau_{2}>\tau_{1}=\tau\left(k_{0}+1\right)+k_{0}\right)$ and $a_{2}>p(\tau+1)$ (see Remark 9.1). Therefore (8.27) implies $\Lambda_{\mathrm{n}}^{2 v}\left(i_{\infty}\right) \subset \Lambda_{\mathrm{n}}^{v}\left(\tau_{\mathrm{n}-1}\right), \forall \mathrm{n} \geqq 1$. By similar arguments we deduce that $\Lambda_{\mathrm{n}}^{2 v, I}\left(i_{\infty}\right) \subset \Lambda_{\mathrm{n}}^{v, I}\left(\tilde{l}_{\mathrm{n}-1}\right)$.

Then we define the $\mu_{j}^{\infty}$ in $(5.12)$, where $\mathrm{m}_{\frac{3}{2}}^{\infty}:=\mathrm{m}_{\frac{3}{2}}\left(i_{\infty}\right), \mathrm{m}_{1}^{\infty}=\mathrm{m}_{1}\left(i_{\infty}\right), \mathrm{m}_{\frac{1}{2}}^{\infty}=$ $\mathrm{m}_{\frac{1}{2}}\left(i_{\infty}\right)$, with $\mathrm{m}_{\frac{3}{2}}, \mathrm{~m}_{1}, \mathrm{~m}_{\frac{1}{2}}$ provided in Proposition 7.18. By (8.16), the sequence $\left(\mathfrak{r}_{j}^{(\mathrm{n})}\left(i_{\infty}\right)\right)_{\mathrm{n} \in \mathbb{N}}$, with $\mathfrak{r}_{j}^{(\mathrm{n})}$ given by Theorem 8.2-(S1) $\left(\right.$ evaluated at $\left.i=i_{\infty}\right)$, is a Cauchy sequence in $|\cdot|^{k_{0}, v}$. Then we define $\mathfrak{r}_{j}^{\infty}:=\lim _{\mathrm{n} \rightarrow \infty} \mathfrak{r}_{j}^{(\mathrm{n})}\left(i_{\infty}\right)$, for any $j \in \mathbb{S}_{0}^{c}$, which satisfies $\left|\mathfrak{r}_{j}^{\infty}-\mathfrak{r}_{j}^{(\mathrm{n})}\left(i_{\infty}\right)\right|^{k_{0}, v} \leqq C \varepsilon v^{-1} N_{\mathrm{n}-1}^{-\mathrm{a}}$ for any $\mathrm{n} \geqq 0$. Then, recalling $\mathfrak{r}_{j}^{(0)}\left(i_{\infty}\right)=0$ and (7.188), the estimates (5.13) hold (here $C=C(S)$ with 
$S$ fixed in (9.3)). Finally one checks (see for example Lemma 8.7 in [6]) that the Cantor set $\mathcal{C}_{\infty}^{v}$ in (5.14)-(5.17) satisfies $\mathcal{C}_{\infty}^{v} \subseteq \mathcal{G}_{\infty}$, with $\mathcal{G}_{\infty}$ defined in (9.11), and Lemma 9.3 implies that $\mathcal{C}_{\infty}^{v} \subseteq \cap_{\mathrm{n} \geqq 0} \mathcal{G}_{\mathrm{n}}$. This concludes the proof of Theorem 5.2.

Funding Open access funding provided by SISSA.

Open Access This article is licensed under a Creative Commons Attribution 4.0 International License, which permits use, sharing, adaptation, distribution and reproduction in any medium or format, as long as you give appropriate credit to the original author(s) and the source, provide a link to the Creative Commons licence, and indicate if changes were made. The images or other third party material in this article are included in the article's Creative Commons licence, unless indicated otherwise in a credit line to the material. If material is not included in the article's Creative Commons licence and your intended use is not permitted by statutory regulation or exceeds the permitted use, you will need to obtain permission directly from the copyright holder. To view a copy of this licence, visit http://creativecommons.org/ licenses/by/4.0/.

Publisher's Note Springer Nature remains neutral with regard to jurisdictional claims in published maps and institutional affiliations.

\section{References}

1. Alazard, T., Baldi, P.: Gravity capillary standing water waves. Arch. Ration. Mech. Anal. 217(3), 741-830, 2015

2. Baldi, P., Berti, M., Haus, E., Montalto, R.: Time quasi-periodic gravity water waves in finite depth. Invent. Math. 214(2), 739-911, 2018

3. Bambusi, D., Berti, M., Magistrelli, E.: Degenerate KAM theory for partial differential equations. J. Differ. Equ. 250(8), 3379-3397, 2011

4. Berti, M., Bolle, P.: A Nash-Moser approach to KAM theory. Fields Institute Communications, special volume "Hamiltonian PDEs and Applications", pp. 255-284, 2015

5. Berti, M., Kappeler, T., Montalto, R.: Large KAM tori for quasi-linear perturbations of KdV. Arch Rational Mech Anal 239, 1395-1500 (2021). https://doi.org/10.1007/ s00205-020-01596-2

6. Berti, M., Montalto, R.: Quasi-periodic standing wave solutions of gravity-capillary water waves, MEMO, Volume 263, 1273, Memoires AMS, ISSN 0065-9266, 2020

7. Constantin, A.: Nonlinear Water Waves with Applications to Wave-Current Interaction and Tsunamis, CBMS-NSF Regional Conf, Series in Applied Math., 81, SIAM, 2011

8. Constantin, A., Ivanov, R.I., Prodanov, E.M.: Nearly-Hamiltonian structure for water waves with constant vorticity. J. Math. Fluid Mech. 10, 224-237, 2008

9. Constantin, A., Strauss, W.: Exact steady periodic water waves with vorticity. Commun. Pure Appl. Math. 57(4), 481-527, 2004

10. Coutand, D., Shkoller, S.: Wellposedness of the free-surface incompressible Euler equations with or without surface tension. J. Am. Math. Soc. 20(3), 829-930, 2007

11. Craig, W., Nicholls, D.: Travelling two and three dimensional capillary gravity water waves. SIAM J. Math. Anal. 32(2), 323-359, 2000. (electronic)

12. Craig, W., Nicholls, D.: Traveling gravity water waves in two and three dimensions. Eur. J. Mech. B Fluids 21(6), 615-641, 2002

13. Craig, W., Sulem, C.: Numerical simulation of gravity waves. J. Comput. Phys. 108(1), 73-83, 1993

14. Dubreil-Jacotin, M.-L.: Sur la détermination rigoureuse des ondes permanentes périodiques d'ampleur finie. J. Math. Pures Appl. 13, 217-291, 1934 
15. Feola, R., Giuliani, F., Procesi, M.: Reducible KAM tori for Degasperis-Procesi equation. Commun. Math. Phys. 377, 1681-1759, 2020

16. Geng, J., You, J.: A KAM theorem for one dimensional Schrödinger equation with periodic boundary conditions. J. Differ. Equ. 209(1), 1-56, 2005

17. Geng, J., You, J.: A KAM theorem for Hamiltonian partial differential equations in higher dimensional spaces. Commun. Math. Phys. 262, 343-372, 2006

18. Gerstner, F.: Theorie der Wellen, Abh. Königl. Böhm. Ges. Wiss, 1802

19. Goyon, R.: Contribution á la théorie des houles. Ann. Sci. Univ. Toulouse 22, 1-55, 1958

20. Grébert, B., Vilaça Da Rocha, V.: Stable and unstable time quasi periodic solutions for a system of coupled NLS equations. Nonlinearity 31(10), 4776-4811, 2018

21. Ifrim, M., TATARU, D.: Two dimensional gravity water waves with constant vorticity: I. Cubic lifespan. Anal. PDE 12(4), 903-967, 2019

22. Iooss, G., Plotnikov, P.: Multimodal standing gravity waves: a completely resonant system. J. Math. Fluid Mech. 7(suppl. 1), S110-S126, 2005

23. Iooss, G., Plotnikov, P.: Small divisor problem in the theory of three-dimensional water gravity waves. Mem. Am. Math. Soc. 200(940), viii+128, 2009

24. Iooss, G., Plotnikov, P.: Asymmetrical tridimensional traveling gravity waves. Arch. Rat. Mech. Anal. 200(3), 789-880, 2011

25. Iooss, G., Plotnikov, P., Toland, J.: Standing waves on an infinitely deep perfect fluid under gravity. Arch. Ration. Mech. Anal. 177(3), 367-478, 2005

26. Lannes, D.: Well-posedness of the water-waves equations. J. Am. Math. Soc. 3, 605654,2005

27. Levi-Civita, T.: Détermination rigoureuse des ondes permanentes d' ampleur finie. Math. Ann. 93, 264-314, 1925

28. Kappeler, T., Liang, Z.: A KAM theorem for the defocusing NLS equation. J. Differ. Equ. 252, 4068-4113, 2012

29. Keady, G., Norbury, J.: On the existence theory for irrotational water waves. Math. Proc. Camb. Philos. Soc. 83(1), 137-157, 1978

30. Martin, C.: Local bifurcation and regularity for steady periodic capillary-gravity water waves with constant vorticity. Nonlinear Anal. Real World Appl. 14(1), 131-149, 2013

31. Maspero, A., Procesi, M.: Long time stability of small finite gap solutions of the cubic Nonlinear Schrödinger equation on $\mathbb{T}^{2}$. J. Differ. Equ. 265(7), 3212-3309, 2018

32. McLeOd, J.B.: The Stokes and Krasovskii conjectures for the wave of greatest height. Stud. Appl. Math. 98(4), 311-333, 1997

33. Nekrasov, A.I.: On steady waves. Izv. Ivanovo-Voznesenk. Politekhn. 3, 1921

34. Plotnikov, P., Toland, J.: Nash-Moser theory for standing water waves. Arch. Ration. Mech. Anal. 159(1), 1-83, 2001

35. Procesi, C., Procesi, M.: A KAM algorithm for the completely resonant nonlinear Schrödinger equation. Adv. Math. 272, 399-470, 2015

36. ReEder, J., SHINBRot, M.: Three-dimensional, nonlinear wave interaction in water of constant depth. Nonlinear Anal. T.M.A. 5(3), 303-323, 1981

37. RüssmanN, H.: Invariant tori in non-degenerate nearly integrable Hamiltonian systems. Regul. Chaotic Dyn. 6(2), 199-204, 2001

38. Stokes, G.: On the theory of oscillatory waves. Trans. Camb. Philos. Soc. 8, 441-455, 1847

39. Struik, D.: Détermination rigoureuse des ondes irrotationelles périodiques dans un canal á profondeur finie. Math. Ann. 95, 595-634, 1926

40. Toland, J.F.: On the existence of a wave of greatest height and Stokes conjecture. Proc. R. Soc. Lond. Ser. A 363(1715), 469-485, 1978

41. WAHLÉn, E.: Steady periodic capillary-gravity waves with vorticity. SIAM J. Math. Anal. 38, 921-943, 2006

42. WAhlÉn, E.: A Hamiltonian formulation of water waves with constant vorticity. Lett. Math. Phys. 79, 303-315, 2007 
43. Wahlén, E.: Steady water waves with a critical layer. J. Differ. Equ. 246, 2468-2483, 2009

44. Wilkening, J., Zhao X.: Spatially quasi-periodic water waves of infinite depth, arXiv:2001.10745, 2020

45. Wilkening, J., ZhaO X.: Quasi-periodic traveling gravity-capillary waves, arXiv:2002.09487, 2020

46. ZaKharov, V.E.: Stability of periodic waves of finite amplitude on the surface of a deep fluid. Zhurnal Prikladnoi Mekhaniki i Teckhnicheskoi Fiziki 9(2), 86-94, 1969

47. ZeIDLER, E.: Existenzbeweis für cnoidal waves unter Berücksichtigung der Oberflächen spannung. Arch. Ration. Mech. Anal 41, 81-107, 1971

48. ZEIDLER, E.: Existenzbeweis fúr permanente Kapillar-Schwerewellen mit allgemeinen Wirbelverteilungen. Arch. Ration. Mech. Anal. 50, 34-72, 1973

M. Berti, L. Franzoi, A. Maspero International School for Advanced Studies (SISSA), Via Bonomea 265,

34136 Trieste

Italy.

M. BERTI

e-mail: berti@ sissa.it

L. FranzoI

e-mail: luca.franzoi@sissa.it

A. Maspero

e-mail: alberto.maspero@sissa.it

(Received April 24, 2020 / Accepted January 18, 2021)

Published online February 25, 2021

(C) The Author(s) (2021) 